\title{
Nutritional interventions focusing on gastrointestinal and metabolic health
}

Citation for published version (APA):

Salden, B. N. H. (2017). Nutritional interventions focusing on gastrointestinal and metabolic health.

[Doctoral Thesis, Maastricht University]. Maastricht University. https://doi.org/10.26481/dis.20170317bs

Document status and date:

Published: 01/01/2017

DOI:

10.26481/dis.20170317bs

Document Version:

Publisher's PDF, also known as Version of record

\section{Please check the document version of this publication:}

- A submitted manuscript is the version of the article upon submission and before peer-review. There can be important differences between the submitted version and the official published version of record.

People interested in the research are advised to contact the author for the final version of the publication, or visit the DOI to the publisher's website.

- The final author version and the galley proof are versions of the publication after peer review.

- The final published version features the final layout of the paper including the volume, issue and page numbers.

Link to publication

\footnotetext{
General rights rights.

- You may freely distribute the URL identifying the publication in the public portal. please follow below link for the End User Agreement:

www.umlib.nl/taverne-license

Take down policy

If you believe that this document breaches copyright please contact us at:

repository@maastrichtuniversity.nl

providing details and we will investigate your claim.
}

Copyright and moral rights for the publications made accessible in the public portal are retained by the authors and/or other copyright owners and it is a condition of accessing publications that users recognise and abide by the legal requirements associated with these

- Users may download and print one copy of any publication from the public portal for the purpose of private study or research.

- You may not further distribute the material or use it for any profit-making activity or commercial gain

If the publication is distributed under the terms of Article $25 \mathrm{fa}$ of the Dutch Copyright Act, indicated by the "Taverne" license above, 
Nutritional interventions focusing on gastrointestinal and metabolic health 
(c) Bouke Salden, Maastricht 2017

All rights reserved. No part of this book may be reproduced or transmitted in any form or by any means, without prior permission in writing by the author, or when appropriate, by the publishers of the publications.

Layout: Tiny Wouters

Cover design: Kira van Landschoot | www.vankira.n |

Print: Giel Nijssen Grafisch Adviesbureau

ISBN: 978-94-6333-129-6

Printing of this thesis was financially supported by Maastricht University, BioActor BV, Nederlandse Vereniging voor Gastroenterologie, Catharina Hospital, Pentax Medical, DSM, Imagelabonline \& Cardiovascular, Dr. Falk Pharma. 


\section{Nutritional interventions focusing on gastrointestinal and metabolic health}

\section{PROEFSCHRIFT}

ter verkriiging van de graad van doctor aan de Universiteit Maastricht, op gezag van de Rector Magnificus, Prof. dr. Rianne M. Letschert, volgens het besluit van het College van Decanen,

in het openbaar te verdedigen op vrijdag 17 maart 2017 om 12.00 uur

door 


\title{
Promotoren
}

Prof. dr. A.A.M. Masclee

\section{Co-promotores}

\author{
Dr. F.J. Troost \\ Dr. S. Possemiers
}

\section{Beoordelingscommissie}

Prof. dr. K. Venema, voorzitter

Dr. D. Jonkers

Prof. dr. C.J.J. Mulder, VUMC

Prof. dr. J. Plat

Prof. dr. R.F. Witkamp, Wageningen Universiteit 
- Big things have small beginnings - 



\section{Contents}

Chapter 1 General introduction

Chapter 2 Randomised clinical study: Aspergillus niger-derived enzyme

digests gluten in the stomach of healthy volunteers

Chapter 3 Paracetamol as a post prandial marker for gastric emptying, a food-drug interaction on absorption

Chapter 4 Reinforcement of intestinal epithelial barrier by arabinoxylans in overweight and obese subjects: a randomised controlled trial

Chapter 5 In vivo bioavailability of hesperidin 2S is improved by micronization: a randomized, double-blind cross-over study

Chapter 6 Randomised clinical trial on the efficacy of hesperidin on validated cardiovascular biomarkers in healthy overweight individuals

Chapter 7 Effect of hesperidin 2S supplementation on systemic and intestinal metabolic parameters in subjects at risk for metabolic syndrome: a randomized controlled trial

Chapter 8 Randomised controlled trial on the intestinal fate and effects of the carotenoid - producing Bacillus strain PDO 1 on gastrointestinal and systemic parameters in overweight individuals-

Chapter 9 General discussion

\section{Addendum}

Summary

Nederlandse samenvatting

Valorisation

List of publications

Curriculum vitae

Dankwoord 


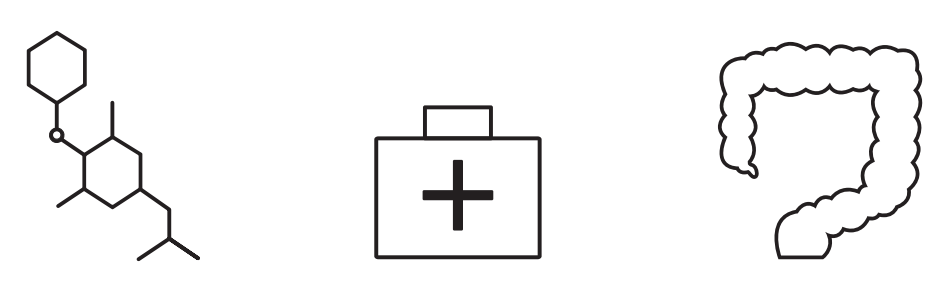


chapter 1

General introduction 


\section{General introduction}

The statement that food may provide therapeutic benefits is clearly not new. The tenet, 'Let food be thy medicine and medicine be thy food' was embraced already about 2500 years ago by Hippocrates. Initially, scientific focus was given on the identification of essential elements (e.g. vitamins) and their role in the prevention and therapy of various dietary deficiency diseases. In recent years, the focus has shifted to explore the role of diet in diseases linked to excess food intake and 'overnutrition'. Furthermore, food is now being examined intensively to identify physiologically active components that potentially have health promoting effects, particularly in chronic diseases or premalignant and malignant disorders or in mild disturbances of organ physiology. This food category is now recognized as 'functional foods'.

In this thesis, we aim to investigate the effects of several nutritional interventions on gastrointestinal and metabolic health. To study these effects we not only focused on healthy, lean subjects but also on obese individuals.

\section{Overweight, obesity and high-fat diet}

The prevalence of overweight, obesity and related diseases (e.g. cardiovascular diseases (CVD) and diabetes mellitus type $2(\mathrm{DM} 2)$ is increasing worldwide. Adults with a body mass index (BMI) of $>25 \mathrm{~kg} / \mathrm{m}^{2}$ are categorized as overweight, those with a BMI of $>30 \mathrm{~kg} / \mathrm{m}^{2}$ as obese.' In the Netherlands in 2015, 50.3\% of the adults aged 20 years and older were overweight and $13.7 \%$ were obese. ${ }^{2}$ Worldwide in 2014 , approximately $39 \%$ of people aged 18 years and over were overweight and $13 \%$ obese. ${ }^{3}$ The increased prevalence of obesity and associated diseases is primarily due to an increase in caloric availability and fat consumption, and a reduction in physical activity in daily life. ${ }^{4}$ Growing evidence supports the idea that also the gut microbiome plays an important role in the development of fat mass and energy homeostasis. ${ }^{5}$ Common features associated with overweight and obesity are alterations in gut microbiota, ${ }^{6,7}$ impaired gut barrier function, ${ }^{8-10}$ (gastrointestinal) and systemic low-grade inflammation, ${ }^{1-14}$ endothelial dysfunction, ${ }^{15}$ hypertension, ${ }^{16}$ glucose homeostasis disorders ${ }^{4}$ and lipid homeostasis disorders. ${ }^{17}$ Obesity and the associated gastrointestinal and systemic metabolic abnormalities are well-recognized risk factors for the development of DM2 and CVD. ${ }^{18-20}$

Obesity imposes a tremendous economic burden on society through increased total direct (treatment obesity-associated diseases) and indirect (productivity losses) medical costs. In Europe, it has been calculated that in general the direct health care costs of obesity account already for up to total $4 \%$ of national health expenditure. It is indicated that indirect health care costs could amount to twice the direct health care costs. ${ }^{21}$ Significant reduction 
in these costs could be accomplished by prevention of overweight or by a delay in the onset of overweight, obesity and associated diseases.

\section{Gastrointestinal and systemic metabolic consequences}

\section{Gut barrier}

Every day, our gastrointestinal tract is exposed to a multitude of different microorganisms and nutrient compounds for subsequent interaction. Balancing this interaction requires a complex control system that is able to prevent entry of antigens and microorganisms into the body, while it allows exchange of molecules between host and environment and absorption of nutrients from the diet. ${ }^{22,23}$ In a healthy organism, the gut barrier is able to fulfill all these needs simultaneously. The gut barrier is a functional unit, organized as a multi-layer system comprising the mucus, epithelial cells, the innate and adaptive immune cells forming the gutassociated lymphoid tissue (GALT) and the gut microbiota. ${ }^{24}$

Mucus protects the epithelium from potentially harmful microorganisms and antigens, but also functions as a lubricating agent for food particles. The mucus layer is dense and only allows low amounts of bacteria to get close to or penetrate the epithelium, which would potentially induce an increase in intestinal permeability with subsequent inflammatory reaction. Furthermore, it is the habitat of the gut microbiota. In addition, the mucus layer serves as food and a binding site for the bacteria, and is probably involved in the selection of specific microbial species, essential for the maintenance of intestinal integrity, homeostasis and function. ${ }^{24,25}$

The gastrointestinal epithelium is a single cell layer which is comprised of different cell types, acting as a physical barrier by inhibiting the passage of luminal contents into the systemic circulation through the formation of complex protein-protein networks that mechanically connect adjacent cells and seal intercellular spaces. ${ }^{26}$ Tight junctions (TJ), adherent junctions (AJ) and desmosomes are the three main junctional complexes connecting adjacent epithelial cells. TJ consist of transmembrane proteins (occludins, claudins, junctional adhesion molecule-A) connected to the actin cytoskeleton via plaque proteins ( $\mathrm{ZO}-1, \mathrm{ZO}-2$ and $\alpha$ catenin) and these proteins regulate paracellular permeability of the epithelium. AJ and desmosomes anchor epithelial cells to one another and confer mechanical strength to the epithelial barrier. ${ }^{27-29}$

Obesity is associated with changes in gut microbiota, which in turn may affect the intestinal permeability via changes in the expression, localization and distribution of TJ proteins, 10, 12,30 and via the endocannabinoid system (eCB). This system consists of endogenous bioactive lipids that are able to alter the distribution of TJ proteins by activating cannabinoid receptors, expressed throughout the gastrointestinal tract. ${ }^{31}$ A 'leak' in the paracellular 
absorption route enables antigens to pass from the intestinal milieu, challenging the immune system to produce an immune response resulting in inflammation and oxidative stress. Gut barrier dysfunction is regarded as an early event or trigger in the development of various intestinal diseases, and of metabolic endotoxemia present in obesity, DM2 and CVD. ${ }^{32,33}$ GALT, gut-associated lymphoid tissue, is a complex network of immune cells representing a functional barrier that protects the 'milieu interieur' from external threats. GALT drives immunological responses to pathogenic microorganisms. Proper functioning of such networks is essential for the maintenance of gut homeostasis, preventing transport of luminal contents towards and into the systemic circulation thus preventing the activation of an immune response and the induction of an inflammatory state. This underlies the pathogenesis of many gastrointestinal diseases, such as inflammatory bowel diseases (IBD) and celiac disease. $^{34}$

\section{Gut microbiota}

The human gut microbiome refers to the entire population of microorganisms colonizing the gut. It is a complex ecosystem that includes at least $10^{14}$ bacteria and also other microbes, such as yeasts and viruses. ${ }^{24}$ It is an organ by itself, which plays an important role within our body, being involved in many functional processes essential for our homeostasis, such as the metabolism of nutrients and drugs, the regulation of many metabolic pathways, the maintenance of epithelial integrity, the modulation of gastrointestinal motility, the stimulation and maturation of both systemic and mucosal immunity, and the production of vitamins and micronutrients. ${ }^{35,36}$ The majority of the gut bacteria is non-pathogenic and co-habit with the gut mucosa in a symbiotic relationship. Intestinal microbiota convert undigested food components into various metabolites, such as short-chain fatty acids (SCFA), which can be further processed by the host and appear to be important signalling molecules. Many of the beneficial physiologic properties of the microbiota can be attributed to the production of these SCFA, particularly acetate, propionate and butyrate. ${ }^{37-39}$

In humans, the interaction between genetic and environmental factors (diet and physical activity) is considered as the main contributor for the development of obesity. Recently, gut microbiota have emerged as a potentially important factor influencing development of overweight. However, the exact mechanisms through which the gut microbiota contribute to obesity are still unclear. It has been suggested that the microbiome has the capacity to increase energy harvested from the diet, and that it is able to influence host energy balance and metabolism by modulating host signaling pathways.,40-42 Furthermore, the gut microbiome might contribute to the development of low-grade inflammation by production of lipopolysaccharide (LPS), a powerful pro-inflammatory molecule from the cell wall of Gram-negative bacteria, which is continuously produced in the host gut with the death and lysis of Gram-negative bacteria. ${ }^{711}$ Today, many questions still remain to be answered: for 
instance whether changes in gut microbiota composition and diversity are a cause or a consequence of the observed pathology.

\section{Low-grade inflammation}

Persistently high circulating levels of inflammatory cytokines (IFN-gamma and TNF-alpha) are often observed in obese humans, and may cause an impaired gut barrier function by affecting TJ proteins. ${ }^{43,44}$ An increased intestinal permeability may lead to translocation of bacterial endotoxins, such as LPS, into the systemic circulation where it is able to activate inflammatory pathways. ${ }^{45}$ Other processes contributing to these elevated LPS levels are an increase in chylomicron-driven LPS transport and a decrease in processes involved in intestinal LPS degradation (alkaline phosphatase activity). ${ }^{12,30,46}$ Additionally, the state of insulin resistance, often observed in obese individuals, promotes inflammation by impairing the anti-inflammatory effects of insulin. ${ }^{47,48}$

\section{Vascular function}

The vascular endothelium is a monolayer of cells between the lumen and the vascular smooth muscle cells. It plays a crucial role in the maintenance of vascular homeostasis by keeping a refined balance between vasodilation and vasoconstriction. ${ }^{49}$ Nitric oxide (NO), generated by endothelial nitric oxide synthase (eNOS), is of pivotal importance in regulating the arterial tone and thus in regulating endothelial function (EF)..$^{50}$ It is able to dilate all types of blood vessels, is considered to be a potent inhibitor of platelet aggregation and adhesion and can also inhibit leukocyte adhesion. ${ }^{51}$ Two important events in atherogenesis are the recruitment and differentiation of circulating monocytes to the endothelium, and the uptake of cholesterol and oxidized low-density lipoproteins (LDL) by tissue macrophages to form lipid-foam cells. Cellular adhesion molecules, such as vascular cell adhesion molecule-1 (VCAM-1), intercellular adhesion molecule-1 (ICAM-1) and E-selectins participate in leukocyte-endothelial interactions and are strongly expressed on macrophages within atherosclerotic plaques. ${ }^{52,53}$ Therefore, based on the combination of its effects, endothelial $\mathrm{NO}$ is considered an important anti-atherogenic defense mechanism in vasculature.

Exposing endothelial cells to obesity and cardiovascular risk factors leads to oxidative stress. As a result, endothelial dysfunction (ED) can occur due to a decreased $\mathrm{NO}$ bioavailability by a reduction in eNOS expression or acceleration in NO degradation. ${ }^{15.51}$ Subsequently, it plays a key role in the development and progression of atherosclerosis. ED is often manifested by an impaired capacity of the vascular endothelium to dilate, which can be quantified by the degree of flow-mediated dilation (FMD), a non-invasive $\mathrm{NO}$-dependent endothelial function assessment. ${ }^{54}$ 
Another aspect of vascular function that can be affected by obesity is blood pressure. Both animal and human studies have revealed a strong relationship between obesity and hypertension. ${ }^{55}$ Several mechanisms have been identified as potential causes of obesityinduced hypertension. As mentioned above, in obesity-associated ED the vasodilating properties of the endothelium are impaired. ${ }^{56}$ Also, increase in both peripheral vasoconstriction and renal tubular sodium reabsorption, ${ }^{57}$ increase in sympathetic activity by obesity-related factors (leptin, hyperinsulinemia, circulating free fatty acids (FFA)), 58-62 overactivation of the renin-angiotensin system (RAS) $)^{63,64}$ and insulin resistance ${ }^{65,66}$ are proposed additional mechanisms mediating hypertension in obesity. A linear relationship exists between blood pressure (BP) and mortality from stroke and ischemic heart disease. ${ }^{67}$ Hence, interventions aimed at preventing or improving obesity would greatly impact the management of ED, obesity-induced hypertension and related diseases.

\section{Glucose and insulin metabolism}

In healthy individuals, plasma glucose is tightly controlled by the balance between glucose absorption from the intestine, production by the liver and uptake and metabolism by peripheral tissues. Insulin serves as the primary regulator of plasma glucose, by increasing glucose uptake from muscle and fat, and by inhibiting hepatic glucose production. Furthermore, it stimulates glycogen synthesis. ${ }^{68}$

Obese individuals are at risk for developing resistance to the cellular actions of insulin. This is characterized by an impaired ability of insulin to inhibit glucose output from the liver and to promote glucose uptake in fat and muscle. ${ }^{68,69}$ Proposed mechanisms, explaining the relationship between increased adipose mass and systemic insulin resistance, focus on dysregulation of interconnected endocrine (FFA, adipokines and cortisol), inflammatory, neural and cell-autonomous pathways. ${ }^{70}$ Insulin resistance is considered the key etiological factor in DM2.

\section{Lipid metabolism}

Numerous metabolic processes are involved in the uptake, transport and storage of lipids. In the intestinal lumen, triglycerides (TG) are lipolyzed into FFA and taken up by the enterocyte, where they are assembled in TG again. Cholesterol is in the enterocyte transformed into cholesterol-esters. TG, cholesterol-esters, phospholipids and apolipoprotein $B$ together form chylomicrons, which enter the systemic circulation. After food-derived TG and FFA reach the liver, TG-rich lipoproteins called very low density lipoproteins (VLDL), are synthesized. Adequate lipolysis of chylomicrons and VLDL by lipoprotein lipase (LPL) delivers FFA to the heart, skeletal muscle and adipose tissue for energy expenditure and storage. The liberated FFA is then taken up by adipocytes and re-synthesized into TG. During the 
process of lipolysis also chylomicron remnants and dense LDL are formed, these products are taken up by the liver. The intestine and liver also play an important role in the reverse cholesterol transport by the synthesis of high-density lipoproteins (HDL). HDL promotes the uptake of cholesterol from peripheral tissues and returns it to the liver. ${ }^{71}$

Typical dyslipidemia in obesity consists of hypertriglyceridemia in combination with an increase in small dense LDL and a low HDL. ${ }^{17}$ In hypertriglyceridemia, increased FFA fluxes to the liver are a consequence of increased FFA release from adipose tissue and reduced FFA clearance from plasma. The increased FFA flux results in hepatic accumulation of TG followed by an increase in VLDL synthesis. Lipolysis of TG-rich lipoproteins is impaired in obesity, due to competition for lipolysis between VLDL and chylomicrons, reduced LPL expression in adipose tissue and reduced LPL activity. Also, HDL metabolism is affected by obesity. An increased number of TG-rich lipoproteins results in an increased exchange of cholesterolesters from HDL for TG from VLDL and LDL. In addition, TG-depleted small dense $\mathrm{LDL}$ is formed by removing TG and phospholipids from LDL. ${ }^{71-73}$ Furthermore, also insulin plays an important role in lipid metabolism as it stimulates LPL activity, facilitates FFA uptake, inhibits hydrolysis of intracellular lipids postprandially, and regulates FFA mobilization from adipose tissue during fasting states. ${ }^{68}$ Dyslipidemia in obesity is an established independent risk factor for the development of CVD. ${ }^{74}$

\section{Nutritional interventions in healthy, overweight and obese individuals}

Prevention or improvement of gastrointestinal and chronic metabolic diseases by a nutritional intervention appears to be a promising approach in health and obesity. At present, a number of dietary strategies are available.

\section{Prolyl endoproteases}

Gluten is a storage protein present in wheat, barley and rye and is exceptionally rich in proline. Proline-cleaving proteases are absent in the human gastrointestinal tract, ensuring long proline-rich gluten peptides to enter the small intestine upon ingestion, subsequently triggering an abnormal immune response in subjects suffering from wheat allergy or coeliac disease. $^{75-77}$ Subjects with non-coeliac gluten sensitivity also experience distress after gluten intake, but no allergic or autoimmune mechanisms are involved in this condition. ${ }^{78-81}$ It has been proposed that in these subjects a gluten-induced activation of the innate immune system occurs. ${ }^{79}$ In general, treatment of these gluten-related disorders is based on excluding gluten-containing cereals from the diet, which is often difficult to comply with as 
gluten-free products may not always be labeled as such or may not always be at hand during social events or travelling. ${ }^{82-84}$

The Aspergillus Niger-derived prolyl endoprotease (AN-PEP) belongs to a family of enzymes having the ability of cleaving at internal proline residues within a peptide. ${ }^{85}$ The use of AN-PEP seems to be a promising approach to digest unintended dietary gluten as in vitro studies have shown that this enzyme is capable of degrading gluten under acidic stomach conditions and is safe for human use. ${ }^{86-89}$ However, in vivo data confirming these promising in vitro results regarding the gastric degradation of gluten are still lacking.

\section{Prebiotics}

Prebiotics are non-digestible compounds that selectively stimulate growth and/or activity of one or a limited number of microbial species in the gut with potential health benefits to the host. ${ }^{90}$ Prebiotic fermentation leads to beneficial host effects by the following major mechanisms: (1) by increasing the relative abundance of beneficial bacteria (i.e. bifidobacteria, lactobacilli), (2) by increasing SCFA production and reducing the production of putrefactive compounds, and (3) by impacting on the gut's immune system. ${ }^{91,92}$ The most common prebiotics used in gut microbiota modulation are the inulins, fructooligosaccharides, various types of galactooligosaccharides and resistant starches. A limitation of these prebiotics is their rapid fermentation in the proximal colon. ${ }^{93.94}$ Dietary carbohydrate is the main fermentable substrate in the proximal colon and as it is degraded during bacterial fermentation, protein takes over as the dominant fermentable substrate towards the distal colon. However, bacterial protein metabolism produces toxic and potentially carcinogenic compounds, playing an important role in the development of distal intestinal chronic diseases. ${ }^{95}$ Thus, there is currently much interest in the development of prebiotics having the ability to persist towards the distal region of the colon.

Arabinoxylans $(A X)$, the most abundant non-digestible carbohydrates present in wheat, form an interesting novel class of potential prebiotics. ${ }^{96-98}$ Due to their structure, AX rely on a whole spectrum of (microbial) enzymes for degradation, resulting in a more distal fermentation and activity. ${ }^{97-99}$ Furthermore, $A X$ exist in different forms, ranging from longchains to enzymatically modified short-chain fractions. ${ }^{100}$ Previous studies indicate that AX structure affects the fermentation pattern and immune modulation, with the highest activity observed for high-molecular weight $A X^{99,101,102}$ Prior in vitro and animal studies have shown promising effects of high-molecular weight $A X$ on several aspects of the gut barrier, ${ }^{93,103-105}$ immune system ${ }^{103}$ and metabolic markers. ${ }^{103}$ To date, no human studies have been published exploring the effect of such $A X$ extract on these parameters. 


\section{Probiotics}

Another proposed nutritional intervention, able to change gut microbiota composition, is the use of probiotics. The internationally endorsed definition of probiotics is live microorganisms that, when administered in adequate amounts, confer a health benefit on the host. ${ }^{106}$ In order to establish an effect, probiotic bacteria will have to survive passage through the gastrointestinal tract. The effects can be classified in three modes of action; they are able to (1) inhibit the action of pathogenic bacteria, (2) stimulate and modulate host defenses and gut barrier, and (3) produce important metabolites for the host. ${ }^{107,108}$ Most probiotic products currently on the market contain lactic acid bacteria, such as lactobacilli and streptococci, or bifidobacteria. ${ }^{109}$ These bacteria are all normal constituents of the human gut microbiota.

In this thesis we investigate the probiotic effects of a Bacillus indicus strain. Bacillus species as probiotics are attractive as they form heat-stable spores. These spores are capable of surviving the gastric barrier without loss of function, are stable to many food-processing steps and can be stored at room temperature. ${ }^{110,111}$ No previous human study investigating the effect of this specific strain on gut microbiota composition, activity and gut barrier is available, but animal and human studies exploring the effect of other bacillus strains have shown beneficial effects. ${ }^{112-117}$

\section{Phytonutrients}

Phytonutrients, also known as phytochemicals, are chemicals produced by plants. These phytochemicals are used to protect the plant from UV light, insects or pests and produces color and other organoleptic characteristics. ${ }^{118}$ Many of these phytochemicals have antioxidant properties and might have the potential to improve health. In this thesis we discuss two different phytonutrients, polyphenols and carotenoids.

Polyphenols are a large group of bioactive plant compounds and are divided into different classes based upon their chemical structure. The gut microbiota plays a critical role in transforming dietary polyphenols into absorbable biologically active species. ${ }^{19}$ Recent animal and human studies have shown that dietary polyphenols are able to modulate the human gut microbiota. ${ }^{120.121}$ In addition, large epidemiological studies link increased consumption of polyphenol-rich foods with reductions in CVD morbidity and mortality. ${ }^{122-124}$ Flavonoids are the largest class of polyphenols, present in many foods of plant origin. ${ }^{125}$ Hesperidin, a flavonoid abundantly present in the peels of citrus fruits, to a large extent reaches the colon intact where it is subsequently deglycosylated by intestinal microbiota to produce the active aglycone hesperitin. ${ }^{126,127}$ Several human intervention studies have shown promising effects of hesperidin on different cardiovascular biomarkers, such as lowgrade inflammation, endothelial function, blood pressure, glucose / insulin metabolism and 
blood lipid profiles. ${ }^{128-132}$ However, in humans hesperidin is poorly soluble, resulting in a limited bioavailability and biological activity. While natural hesperidin products contain high concentrations of the most active form of hesperidin (hesperitin-7-O-rutinoside 2S), typical hesperidin extracts on the market mainly contain the less active $2 \mathrm{R}$ enantiomer. In this thesis, we study the bioavailability and biological activity of a hesperidin 2S extract with a specific enantiomer configuration similar to natural hesperidin.

Carotenoids are organic pigments, synthesized mainly in plants but have also been isolated from yeasts, fungi, marine algae, micro-algae and some species of bacteria. Humans are not able to synthesize carotenoids themselves, and therefore must absorb them from their diet. The incidence of CVD is notably reduced in areas where a 'Mediterranean diet', containing large amounts of fruit and vegetables, and thus carotenoids, predominates. ${ }^{133,134}$ In part, the protective effects of carotenoids seem to be related to their potent antioxidant activity. A link between carotenoid intake and protection from $L D L$ oxidation, atherosclerotic progression, hypertension, low-grade inflammation and endothelial dysfunction has been shown. ${ }^{135-137}$ The majority of commercially available carotenoids are derived from plantbased materials. However, the quality and consistency of the end product varies tremendously according to the plant growth conditions. ${ }^{138,139}$ In addition, these carotenoids are often rapidly degraded in the human gastrointestinal tract by the action of gastric juice. As indicated before, carotenoids can also be synthesized by some species of bacteria. Of specific interest are carotenoids produced by a variety of spore-forming bacillus strains, as these strains are able to deliver gastric-stable carotenoids with equal or even higher bioavailability levels than well-established sources of dietary carotenoids in use today. ${ }^{140} \mathrm{In}$ addition, in an in vitro model these bacterial carotenoids showed to have a 10-fold higher antioxidant activity compared to lycopene, one of the most potent antioxidants known (data not published yet). However, no human data regarding the bioavailability, antioxidant activity and systemic effects of these bacterial carotenoids are available up to now. 


\section{Aim and outline of the thesis}

In this thesis, we aimed to investigate the effects of nutritional interventions on gastrointestinal and metabolic health in healthy, overweight and obese individuals. The efficacy of AN-PEP in the gastric degradation of gluten was assessed in healthy (lean) individuals. We chose to study the effect of several other nutritional interventions in overweight and obese subjects. We consider overweight and obesity as "mild disease", associated with a broad spectrum of well-recognized risk factors for the development of chronic metabolic diseases such as CVD and DM2. Figure 1.1 gives an overview of these risk factors, the (inter)relationship between obesity, high-fat diet and risk factors, and the chapters in which each risk factor is measured and discussed.

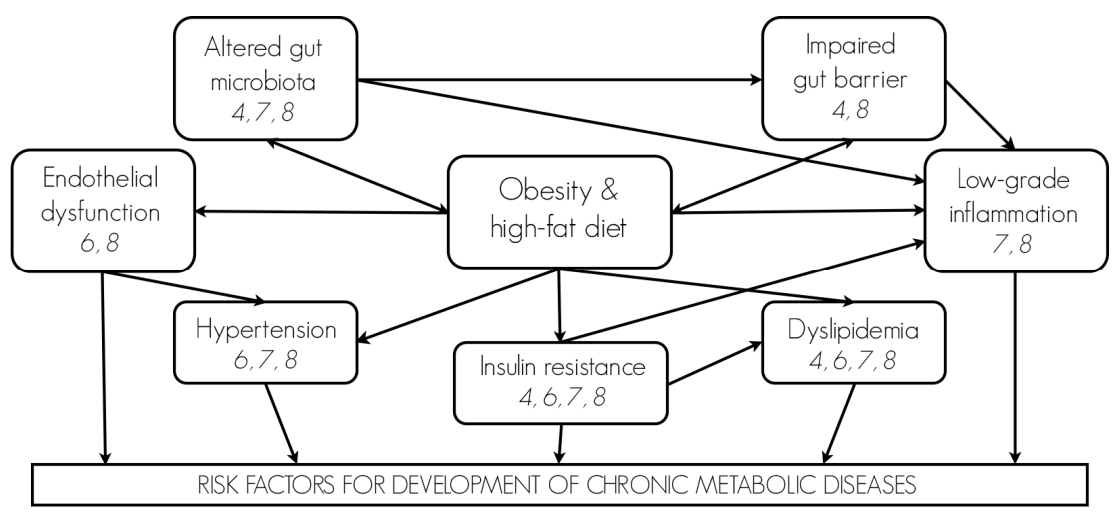

Figure 1.1 Schematic overview of (inter)relationship between obesity, high-fat diet and associated risk factors. The numbers below a risk factor demonstrate the chapters in which the respective risk factor is measured and discussed.

The first part of this thesis (Chapters 2 \& 3) focuses on investigating the efficacy of AN-PEP on gastric gluten degradation in healthy individuals. Furthermore, also the effect of meal caloric density and gastric emptying rate on the enzyme's efficacy was assessed (Chapter 2). In order to display a systemic (patho)biological effect, a compound has to be absorbed from the gastrointestinal tract. The onset of systemic effects depends on the rate of absorption and on gastric emptying rate. In Chapter 3 we critically evaluate the use of paracetamol as a non-invasive method for measuring gastric emptying rate.

The second part of this thesis (Chapter 4-8) explores the impact of different nutritional compounds on parameters of gut and systemic metabolism in overweight and obese, yet healthy, individuals. In Chapter 4 we investigate the effects of 6 weeks daily supplementation of $A X$ on the gut barrier. The study was specifically designed to integrate 
both the analysis of TJ proteins structure and functioning of this barrier. The aspects of the gut barrier we studied include gene transcription and protein expression of $\mathrm{TJ}$ proteins in sigmoid colon mucosa, gastrointestinal permeability, microbial community composition and its metabolic activity. In addition, we also assessed whether AX are capable of modulating the immune response. Another nutritional compound we studied is hesperidin, a flavonoid found in citrus fruits. In humans, low solubility of hesperidin limits its bioavailability and biological activity. In Chapter 5 we studied the in vivo bioavailability of hesperidin. First, we compared the bioavailability of a single intake of standard hesperidin with a hesperidin $2 S$ extract, which was specifically developed for its improved bioavailability. Secondly, we aimed to study the bioavailability of this hesperidin extract in more detail by conducting a placebo controlled trial in which participants received products for 5 consecutive days. Chapter 6 investigates the potential role of hesperidin $2 S$ in the regulation of endothelial function and blood pressure, while Chapter 7 reveals whether it has a role in glucose and insulin metabolism. Furthermore, local effects of hesperidin $2 S$ on gastrointestinal environment were assessed. The last nutritional intervention we performed relates to PDO1, a Bacillus indicus strain known to produce in vitro high amounts of carotenoids. Carotenoids are lipophilic antioxidants, and a potential preventive role of these antioxidants in the development of CVD has been proposed. Furthermore, in vitro data indicate that PDOI itself is able to modulate composition of gut microbiota. Hence, both the effects of the bacillus strain on microbiota composition and functioning and the effects of the carotenoids on CVD risk markers were explored (Chapter 8).

Finally, Chapter 9 summarizes the main findings of all studies presented in this thesis, and discusses the new insights, future perspectives and potential implications for further research and future applications. 


\section{References}

1. Finkelstein EA, Khaviou OA, Thompson $\mathrm{H}$, et al. Obesity and severe obesity forecasts through 2030. Am J Prev Med. 2012;42(6):563-70.

2. Gezondheidsmonitor GGD'en CeR. Gezondheidsmonitor Volwassenen. In: RIVM, editor. 2015

3. Organization WH. Obesity and overweight. Fact sheet No. 211. http://www.who.int/mediacentre/ factsheets/fs3 1 1/en/: World Health Organization Media Centre; 2015

4. Kahn SE, Hull RL, Utzschneider KM. Mechanisms linking obesity to insulin resistance and type 2 diabetes. Nature. 2006:444(7121):840-6.

5. Backhed F, Ding H, Wang $T$, et al. The gut microbiota as an environmental factor that regulates fat storage. Proc Natl Acad Sci U S A. 2004;10 1(44):15718-23.

6. Ley RE. Obesity and the human microbiome. Curr Opin Gastroenterol. 2010;26(1):5-1 1

7. Cani PD, Neyrinck AM, Fava F, et al. Selective increases of bifidobacteria in gut microflora improve highfat-diet-induced diabetes in mice through a mechanism associated with endotoxaemia. Diabetologia. 2007:50( 1 1):2374-83.

8. Sekirov I, Russell SL, Antunes LC, Finlay BB. Gut microbiota in health and disease. Physiol Rev. 2010;90(3):859-904.

9. Cani PD, Possemiers S, Van de Wiele T, et al. Changes in gut microbiota control inflammation in obese mice through a mechanism involving GLP-2-driven improvement of gut permeability. Gut. 2009:58(8): 1091-103.

10. Turner JR. Intestinal mucosal barrier function in health and disease. Nat Rev Immunol. 2009;9(1 1): 799-809.

11. Cani PD, Amar J, Iglesias MA, et al. Metabolic endotoxemia initiates obesity and insulin resistance. Diabetes. 2007:56(7): 1761-72.

12. Cani $P D$, Bibiloni $R$, Knauf $C$, et al. Changes in gut microbiota control metabolic endotoxemia-induced inflammation in high-fat diet-induced obesity and diabetes in mice. Diabetes. 2008:57(6): 1470-81.

13. Hotamisligil GS. Inflammation and metabolic disorders. Nature. 2006;444(7121):860-7.

14. de La Serre CB, Ellis CL, Lee J, et al. Propensity to high-fat diet-induced obesity in rats is associated with changes in the gut microbiota and gut inflammation. Am J Physiol Gastrointest Liver Physiol. 2010;299(2):G440-8.

15. Campia U, Tesauro M, Cardillo C. Human obesity and endothelium-dependent responsiveness. $\mathrm{Br} J$ Pharmacol. 2012;165(3):561-73

16. Kang YS. Obesity associated hypertension: new insights into mechanism. Electrolyte Blood Press. 2013; 1 1(2):46-52.

17. Franssen R, Monajemi H, Stroes ES, Kastelein JJ. Obesity and dyslipidemia. Med Clin North Am. 201 1:95(5):893-902.

18. Van Gaal LF, Mertens IL, De Block CE. Mechanisms linking obesity with cardiovascular disease. Nature. 2006:444(7121):875-80.

19. Wilson PW, D'Agostino RB, Parise $\mathrm{H}$, et al. Metabolic syndrome as a precursor of cardiovascular disease and type 2 diabetes mellitus. Circulation. 2005; 1 12(20):3066-72.

20. Gunderson EP, Lewis CE, Tsai AL, et al. A 20-year prospective study of childbearing and incidence of diabetes in young women, controlling for glycemia before conception: the Coronary Artery Risk Development in Young Adults (CARDIA) Study. Diabetes. 2007:56(12):2990-6.

21. Organization WH. Obesity: preventing and managing the global epidemic. Report of a WHO Consultation. WHO Technical Report Series, No. 894: World Health Organization; 2000. Available from: http://www.who.int/nutrition/publications/obesity/en/index.html.

22. Brandtzaeg $P$. The gut as communicator between environment and host: immunological consequences. Eur $J$ Pharmacol. 201 1:668 Suppl 1:S16-32.

23. Bischoff SC, Barbara G, Buurman W, et al. Intestinal permeability--a new target for disease prevention and therapy. BMC Gastroenterol. 2014;14:189. 
24. Viggiano D, laniro G, Vanella G, et al. Gut barrier in health and disease: focus on childhood. Eur Rev Med Pharmacol Sci. 2015; 19(6):1077-85.

25. Ouwerkerk JP, de Vos WM, Belzer C. Glycobiome: bacteria and mucus at the epithelial interface. Best Pract Res Clin Gastroenterol. 2013;27(1):25-38.

26. Moens E, Veldhoen M. Epithelial barrier biology: good fences make good neighbours. Immunology. 2012;135(1):1-8

27. Naydenov NG, Ivanov Al. Adducins regulate remodeling of apical junctions in human epithelial cells. Mol Biol Cell. 2010;21(20):3506-17.

28. Ulluwishewa D, Anderson RC, McNabb WC, et al.. Regulation of tight junction permeability by intestinal bacteria and dietary components. J Nutr. 2011 1; 141(5):769-76.

29. Fasano A. Zonulin and its regulation of intestinal barrier function: the biological door to inflammation, autoimmunity, and cancer. Physiol Rev. 201 1;91(1):151-75.

30. Brun P, Castagliuolo I, Di Leo V, et al. Increased intestinal permeability in obese mice: new evidence in the pathogenesis of nonalcoholic steatohepatitis. Am J Physiol Gastrointest Liver Physiol. 2007;292(2):G5 18-25.

31. Muccioli GG, Naslain D, Backhed F, et al. The endocannabinoid system links gut microbiota to adipogenesis. Mol Syst Biol. 2010;6:392

32. Hotamisligil GS, Erbay E. Nutrient sensing and inflammation in metabolic diseases. Nat Rev Immunol. 2008;8(12):923-34.

33. Shoelson SE, Goldfine AB. Getting away from glucose: fanning the flames of obesity-induced inflammation. Nat Med. 2009; 15(4):373-4.

34. Constantinovits M, Sipos F, Molnar B, Tulassay Z, Muzes G. Organizer and regulatory role of colonic isolated lymphoid follicles in inflammation. Acta Physiol Hung. 2012;99(3):344-52.

35. Guinane CM, Cotter PD. Role of the gut microbiota in health and chronic gastrointestinal disease: understanding a hidden metabolic organ. Therap Adv Gastroenterol. 2013;6(4):295-308.

36. Sommer F, Backhed F. The gut microbiota--masters of host development and physiology. Nat Rev Microbiol. 2013; 1 1 (4):227-38.

37. Soldavini J, Kaunitz JD. Pathobiology and potential therapeutic value of intestinal short-chain fatty acids in gut inflammation and obesity. Dig Dis Sci. 2013;58(10):2756-66.

38. Duncan SH, Louis $\mathrm{P}$, Thomson JM, Flint HJ. The role of $\mathrm{pH}$ in determining the species composition of the human colonic microbiota. Environ Microbiol. 2009; 1 1(8):21 12-22.

39. Tedelind S, Westberg F, Kierrulf M, Vidal A. Anti-inflammatory properties of the short-chain fatty acids acetate and propionate: a study with relevance to inflammatory bowel disease. World J Gastroenterol. 2007; 13(20):2826-32.

40. Turnbaugh PJ, Ley RE, Mahowald MA, et al. An obesity-associated gut microbiome with increased capacity for energy harvest. Nature. 2006;444(7122):1027-31.

41. Hildebrandt MA, Hoffmann C, Sherrill-Mix SA, et al. High-fat diet determines the composition of the murine gut microbiome independently of obesity. Gastroenterology. 2009; 137(5): 1716-24 e 1-2.

42. Backhed F, Manchester JK, Semenkovich CF, Gordon Jl. Mechanisms underlying the resistance to dietinduced obesity in germ-free mice. Proc Natl Acad Sci U.S.A.. 2007; 104(3):979-84.

43. Das UN. Is obesity an inflammatory condition? Nutrition. 2001; 17(1 1-12):953-66.

44. Bruewer M, Luegering A, Kucharzik T, Parkos CA, Madara JL, Hopkins AM, et al. Proinflammatory cytokines disrupt epithelial barrier function by apoptosis-independent mechanisms. J Immunol. 2003; 171(1 1):6164-72.

45. Medzhitov R, Horng T. Transcriptional control of the inflammatory response. Nat Rev Immunol. 2009:9(10):692-703

46. Delzenne NM, Neyrinck AM, Cani PD. Modulation of the gut microbiota by nutrients with prebiotic properties: consequences for host health in the context of obesity and metabolic syndrome. Microb Cell Fact. $2011 ; 10$ Suppl 1:S10. 
47. Dandona $P$, Aljada A, Mohanty $P$, et al. Insulin inhibits intranuclear nuclear factor kappaB and stimulates IkappaB in mononuclear cells in obese subjects: evidence for an anti-inflammatory effect? I Clin Endocrinol Metab. 2001;86(7):3257-65.

48. Aljada A, Ghanim H, Mohanty P, Kapur N, Dandona P. Insulin inhibits the pro-inflammatory transcription factor early growth response gene-1 (Egr)-1 expression in mononuclear cells (MNC) and reduces plasma tissue factor (TF) and plasminogen activator inhibitor-1 (PAl-1) concentrations. J Clin Endocrinol Metab. 2002;87(3): 1419-22.

49. Kasprzak JD, Klosinska M, Drozdz J. Clinical aspects of assessment of endothelial function. Pharmacol Rep. 2006;58 Suppl:33-40

50. Mudau M, Genis A, Lochner A, Strijdom H. Endothelial dysfunction: the early predictor of atherosclerosis. Cardiovasc J Afr. 2012;23(4):222-31.

51. Forstermann U, Munzel T. Endothelial nitric oxide synthase in vascular disease: from marvel to menace. Circulation. 2006; $113(13): 1708-14$.

52. Jublanc C, Beaudeux JL, Aubart F, et al. Serum levels of adhesion molecules ICAM- 1 and VCAM- 1 and tissue inhibitor of metalloproteinases, TIMP-1, are elevated in patients with autoimmune thyroid disorders: relevance to vascular inflammation. Nutrition, metabolism, and cardiovascular diseases : Nutr Metab Cardiovasc Dis. 201 1;21(10):817-22.

53. Ridker PM, Buring JE, Rifai N. Soluble P-selectin and the risk of future cardiovascular events. Circulation. 200 1; 103(4):491-5.

54. Deanfield JE, Halcox JP, Rabelink TJ. Endothelial function and dysfunction testing and clinical relevance. Circulation. 2007; 1 15(10): 1285-95.

55. Hall JE. The kidney, hypertension, and obesity. Hypertension. 2003;41(3 P+ 2):625-33.

56. Jonk AM, Houben AJ, de Jongh RT, et al.. Microvascular dysfunction in obesity: a potential mechanism in the pathogenesis of obesity-associated insulin resistance and hypertension. Physiology. 2007:252-60. PubMed PMID: 17699878.

57. Vaz M, Jennings $G$, Turner $A$, et al. Regional sympathetic nervous activity and oxygen consumption in obese normotensive human subjects. Circulation. 1997;96(10):3423-9.

58. Carlyle M, Jones $\mathrm{OB}$, Kuo JJ, Hall JE. Chronic cardiovascular and renal actions of leptin: role of adrenergic activity. Hypertension. 2002;39(2 Pt 2):496-501.

59. Rahmouni K, Morgan DA, Morgan GM, et al. Hypothalamic PI3K and MAPK differentially mediate regional sympathetic activation to insulin. J Clin Invest. 2004; 1 14(5): 652-8.

60. Rahmouni K, Haynes WG, Morgan DA, Mark AL. Role of melanocortin-4 receptors in mediating renal sympathoactivation to leptin and insulin. J Neurosci 2003;23( 14):5998-6004.

61. Nielsen S, Guo Z, Johnson CM, Hensrud DD, Jensen MD. Splanchnic lipolysis in human obesity. The J Clin Invest. 2004; $113(1$ 1): 1582-8.

62. Alvarez GE, Beske SD, Ballard TP, Davy KP. Sympathetic neural activation in visceral obesity. Circulation. 2002; 106(20):2533-6

63. Rahmouni K, Mark AL, Haynes WG, Sigmund CD. Adipose depot-specific modulation of angiotensinogen gene expression in diet-induced obesity. Am J Physiol Endocrinol Metab. 2004;286(6):E891-5.

64. Barton M, Carmona $\mathrm{R}$, Morawietz $\mathrm{H}$, et al. Obesity is associated with tissue-specific activation of renal angiotensin-converting enzyme in vivo: evidence for a regulatory role of endothelin. Hypertension. 2000;35( 1 P+ 2):329-36

65. Mykkanen L, Haffner SM, Ronnemaa T, Bergman RN, Laakso M. Low insulin sensitivity is associated with clustering of cardiovascular disease risk factors. Am J Epidemiol. 1997; 146(4):315-21.

66. Saad MF, Rewers M, Selby J, et al. Insulin resistance and hypertension: the Insulin Resistance Atherosclerosis study. Hypertension. 2004;43(6): 1324-31.

67. Lewington S, Clarke R, Qizilbash N, Peto R, Collins R, Prospective Studies C. Age-specific relevance of usual blood pressure to vascular mortality: a meta-analysis of individual data for one million adults in 61 prospective studies. Lancet. 2002;360(9349): 1903-13. 
68. Saltiel AR, Kahn CR. Insulin signalling and the regulation of glucose and lipid metabolism. Nature. 2001;414(6865):799-806.

69. Hribal ML, Oriente F, Accili D. Mouse models of insulin resistance. Am J Physiol Endocrinol Metab. 2002;282(5):E977-81.

70. Qatanani M, Lazar MA. Mechanisms of obesity-associated insulin resistance: many choices on the menu. Genes Dev. 2007;21(12): 1443-55.

71. Klop B, Elte JW, Cabezas MC. Dyslipidemia in obesity: mechanisms and potential targets. Nutrients. 2013;5(4):1218-40.

72. Mook S, Halkes CJM, Bilecen S, Cabezas MC. In vivo regulation of plasma free fatty acids in insulin resistance. Metabolism. 2004;53(9): 1 197-201.

73. Biorntorp P, Bergman H, Varnauskas E. Plasma free fatty acid turnover rate in obesity. Acta Med Scand. 1969; 185(4):351-6.

74. McBride P. Triglycerides and risk for coronary artery disease. Curr Atheroscler Rep. 2008; 10(5): 386-90

75. Piper JL, Gray GM, Khosla C. Effect of prolyl endopeptidase on digestive-resistant gliadin peptides in vivo. J Pharmacol Exp Ther. 2004;31 1(1):213-9.

76. Green PH, Cellier C. Celiac disease. N Engl J Med. 2007;357(17):1731-43.

77. Fasano A, Sapone A, Zevallos V, Schuppan D. Nonceliac gluten sensitivity. Gastroenterology. 2015; 148(6): 1 195-204

78. Sapone A, Bai JC, Ciacci C, et al. Spectrum of gluten-related disorders: consensus on new nomenclature and classification. BMC Med. 2012;10:13.

79. Sapone A, Lammers KM, Casolaro V, et al. Divergence of gut permeability and mucosal immune gene expression in two gluten-associated conditions: celiac disease and gluten sensitivity. BMC Med. 201 1;9:23.

80. Sapone A, Lammers KM, Mazzarella G, et al. Differential mucosal IL-17 expression in two gliadin-induced disorders: gluten sensitivity and the autoimmune enteropathy celiac disease. Int Arch Allergy Immunol. 2010; 152(1):75-80.

81. Ferguson A, Gillett H, Humphreys K, Kingstone K. Heterogeneity of celiac disease: clinical, pathological, immunological, and genetic. Ann N Y Acad Sci. 1998;859: $112-20$.

82. Lerner A. New therapeutic strategies for celiac disease. Autoimmun Rev. 2010;9(3): 144-7.

83. Sollid LM, Khosla C. Future therapeutic options for celiac disease. Nature clinical practice Gastroenterol Hepatol. 2005;2(3): 140-7.

84. Sollid LM, Khosla C. Novel therapies for coeliac disease. J Int Med. 201 1;269(6):604-13.

85. Gass J, Khosla C. Prolyl endopeptidases. Cell Mol Life Sci. 2007;64(3):345-55.

86. Stepniak D, Spaenii-Dekking L, Mitea C, et al. Highly efficient gluten degradation with a newly identified prolyl endoprotease: implications for celiac disease. Am J Physiol Gastrointest Liver Physiol. 2006;291(4):G621-9.

87. ANSES - French Agency for Food EaOHS. AVIS de l'Anses relatif à une demande d'autorisation de mise sur le marché d'un nouvel aliment ou d'un ingrédient alimentaire: préparation enzymatique contenant une activité protéase pour une utilisation dans les compléments alimentaires. 2014. Available from: https://www.anses.fr/.

88. Mitea C, Havenaar R, Drijfhout JW, et al. Efficient degradation of gluten by a prolyl endoprotease in a gastrointestinal model: implications for coeliac disease. Gut. 2008;57(1): 25-32.

89. Tack GJ, van de Water JM, Bruins MJ, et al. Consumption of gluten with gluten-degrading enzyme by celiac patients: a pilot-study. World J Gastroenterol. 2013; 19(35):5837-47.

90. Gibson GR, Roberfroid MB. Dietary modulation of the human colonic microbiota: introducing the concept of prebiotics. The Journal of nutrition. 1995 Jun; 125(6): 140 1-12. PubMed PMID: 7782892.

91. Valcheva R, Dieleman LA. Prebiotics: Definition and protective mechanisms. Best Pract Res Clin Gastroenterol. 2016;30(1):27-37.

92. Macfarlane GT, Steed H, Macfarlane S. Bacterial metabolism and health-related effects of galactooligosaccharides and other prebiotics. J Appl Microbiol. 2008; 104(2):305-44. 
93. Van den Abbeele P, Gerard P, Rabot S, et al. Arabinoxylans and inulin differentially modulate the mucosal and luminal gut microbiota and mucin-degradation in humanized rats. Environ Microbiol. 201 1;13(10): 2667-80.

94. Kolida S, Tuohy K, Gibson GR. Prebiotic effects of inulin and oligofructose. Brit J Nutr. 2002;87: S193-S7.

95. Nyangale EP, Mottram DS, Gibson GR. Gut microbial activity, implications for health and disease: the potential role of metabolite analysis. J Proteome Res. 2012; 1 1(12):5573-85.

96. Pastell H, Westermann P, Meyer AS, Tuomainen P, Tenkanen M. In vitro fermentation of arabinoxylanderived carbohydrates by bifidobacteria and mixed fecal microbiota. I Agric Food Chem. 2009:57(18):8598-606.

97. Grootaert C, Van den Abbeele P, Marzorati M, et al. Comparison of prebiotic effects of arabinoxylan oligosaccharides and inulin in a simulator of the human intestinal microbial ecosystem. FEMS Microbiol Ecol. 2009;69(2):23 1-42.

98. Vardakou M, Palop CN, Christakopoulos $P$, et al. Evaluation of the prebiotic properties of wheat arabinoxylan fractions and induction of hydrolase activity in gut microflora. Int J Food Microbiol. 2008; 123(1-2): 166-70

99. Hughes SA, Shewry PR, Li L, Gibson GR, Sanz ML, Rastall RA. In vitro fermentation by human fecal microflora of wheat arabinoxylans. J Agric Food Chem. 2007;55( 11 1):4589-95.

100. Maes C, Delcour JA. Structural characterisation of water-extractable and water-unextractable arabinoxylans in wheat bran. J Cereal Sci. 2002;35(3):315-26.

101. Pollet A, Van Craeyveld V, Van de Wiele T, et al. In vitro fermentation of arabinoxylan oligosaccharides and low molecular mass arabinoxylans with different structural properties from wheat (Triticum aestivum L.) bran and psyllium (Plantago ovata Forsk) seed husk. J Agricul Food Chem. 2012;60(4):946-54.

102. Monobe M, Ema K, Kato F, Maeda-Yamamoto M. Immunostimulating activity of a crude polysaccharide derived from green tea (Camellia sinensis) extract. J Agric Food Chem. 2008;56(4): 1423-7.

103. Neyrinck AM, Possemiers S, Druart C, et al. Prebiotic effects of wheat arabinoxylan related to the increase in bifidobacteria, Roseburia and Bacteroides/Prevotella in diet-induced obese mice. PloS One. $2011 ; 6(6): e 20944$

104. Van den Abbeele P, Venema K, Van de Wiele T, Verstraete W, Possemiers S. Different human gut models reveal the distinct fermentation patterns of Arabinoxylan versus inulin. I Agric Food Chem. 2013;61(41):9819-27.

105. Chen H, Wang W, Degroote J, et al. Arabinoxylan in wheat is more responsible than cellulose for promoting intestinal barrier function in weaned male piglets. The J Nutr. 2015; 145(1):51-8.

106. Caselli M, Cassol F, Calo G, et al. Actual concept of "probiotics": is it more functional to science or business? World J Gastroenterol. 2013;19(10): 1527-40.

107. Oelschlaeger TA. Mechanisms of probiotic actions - A review. Int J Med Microbiol. 20 10;300(1):57-62.

108. Gionchetti P, Rizzello F, Campieri M. Probiotics in gastroenterology. Curr Opin Gastroenterol. 2002; 18(2):235-9.

109. Penner R, Fedorak RN, Madsen KL. Probiotics and nutraceuticals: non-medicinal treatments of gastrointestinal diseases. Curr Opin Pharmacol. 2005;5(6):596-603

1 10. Barbosa TM, Serra CR, La Ragione RM, Woodward MJ, Henriques AO. Screening for bacillus isolates in the broiler gastrointestinal tract. Appl Environ Microbiol. 2005;71(2):968-78.

1 11. Spinosa MR, Braccini T, Ricca E, De Felice M, Morelli L, Pozzi G, et al. On the fate of ingested Bacillus spores. Res Microbiol. 2000; 151(5):36 1-8.

112. Sanders ME. Probiotics: considerations for human health. Nutrition reviews. 2003 Mar;61(3):91-9.

113. Cutting SM. Bacillus probiotics. Food Microbiol. 201 1;28(2):214-20.

114. Nyangale EP, Farmer S, Cash HA, et al.. Bacillus coagulans GBI-30, 6086 Modulates Faecalibacterium prausnitzii in Older Men and Women. J Nutr. 2015; 145(7): 1446-52.

115. Jayaraman S, Thangavel G, Kurian H, et al. Bacillus subtilis PB6 improves intestinal health of broiler chickens challenged with Clostridium perfringens-induced necrotic enteritis. Poult Sci. 2013;92(2):370-4. 
1 16. Kim HS, Park H, Cho IY, Paik HD, Park E. Dietary supplementation of probiotic Bacillus polyfermenticus, Bispan strain, modulates natural killer cell and T cell subset populations and immunoglobulin $G$ levels in human subjects. J Med Food. 2006 Fall;9(3):321-7.

117. Prieto ML, O'Sullivan L, Tan SP, et al. Evaluation of the efficacy and safety of a marine-derived Bacillus strain for use as an in-feed probiotic for newly weaned pigs. PloS One. 2014;9(2):e88599.

1 18. Bennett RN WR. Secondary metabolites in plant defense mechanisms. New Phytol. 1994; 127:617-33.

119. Clifford MN. Diet-derived phenols in plasma and tissues and their implications for health. Planta Med. 2004;70(12): 1 103-14.

120. Fiesel A, Gessner DK, Most E, Eder K. Effects of dietary polyphenol-rich plant products from grape or hop on pro-inflammatory gene expression in the intestine, nutrient digestibility and faecal microbiota of weaned pigs. BMC Vet Res. 2014;10:196.

121. Queipo-Ortuno MI, Boto-Ordonez M, Murri M, et al. Influence of red wine polyphenols and ethanol on the gut microbiota ecology and biochemical biomarkers. Am J Clin Nutr. 2012;95(6): 1323-34.

122. Hertog MG, Feskens EJ, Hollman PC, Katan MB, Kromhout D. Dietary antioxidant flavonoids and risk of coronary heart disease: the Zutphen Elderly Study. Lancet. 1993;342(8878): 1007- 11

123. Hertog MG, Kromhout D, Aravanis $C$, et al. Flavonoid intake and long-term risk of coronary heart disease and cancer in the seven countries study. Arch Intern Med. 1995; 155(4):38 1-6.

124. Mink PJ, Scrafford CG, Barraj LM, et al. Flavonoid intake and cardiovascular disease mortality: a prospective study in postmenopausal women. Am J Clin Nutr. 2007;85(3):895-909.

125. Harnly JM, Doherty RF, Beecher GR, et al. Flavonoid content of U.S. fruits, vegetables, and nuts. J Agric Food Chem. 2006;54(26):9966-77.

126. Cermak R, Breves GM. In vitro degradation of the flavonol quercetin and of quercetin glycosides in the porcine hindgut. Arch Anim Nutr. 2006:60(2): 180-9.

127. Erlund I, Meririnne E, Alfthan G, Aro A. Plasma kinetics and urinary excretion of the flavanones naringenin and hesperetin in humans after ingestion of orange juice and grapefruit juice. J Nutr. 2001;131(2):235-41.

128. Rizza S, Muniyappa R, lantorno M, et al. Citrus polyphenol hesperidin stimulates production of nitric oxide in endothelial cells while improving endothelial function and reducing inflammatory markers in patients with metabolic syndrome. J Clin Endocrinol Metab. 201 1;96(5):E782-92.

129. Morand C, Dubray C, Milenkovic D, et al. Hesperidin contributes to the vascular protective effects of orange juice: a randomized crossover study in healthy volunteers. Am J Clin Nutr. 201 1;93(1):73-80.

130. Milenkovic D, Deval C, Dubray C, Mazur A, Morand C. Hesperidin displays relevant role in the nutrigenomic effect of orange juice on blood leukocytes in human volunteers: a randomized controlled crossover study. PloS One. 201 1;:6(1 1):e26669.

131. Kurowska EM, Spence JD, Jordan J, Wetmore S, Freeman DJ, Piche LA, et al. HDL-cholesterol-raising effect of orange juice in subjects with hypercholesterolemia. Am J Clin Nutr. 2000;72(5): 1095-100.

132. Miwa Y, Mitsuzumi H, Sunayama T, et al. Glucosyl hesperidin lowers serum triglyceride level in hypertriglyceridemic subjects through the improvement of very low-density lipoprotein metabolic abnormality. J Nutr Sci Vitaminol. 2005;51(6):460-70.

133. Serra-Majem L, Roman B, Estruch R. Scientific evidence of interventions using the Mediterranean diet: a systematic review. Nutr Rev. 2006:64(2 Pt 2):S27-47.

134. Muller-Nordhorn J, Binting S, Roll S, Willich SN. An update on regional variation in cardiovascular mortality within Europe. Eur Heart J. 2008;29(10): 1316-26.

135. Hozawa A, Jacobs DR, Jr., Steffes MW, et al. Relationships of circulating carotenoid concentrations with several markers of inflammation, oxidative stress, and endothelial dysfunction: the Coronary Artery Risk Development in Young Adults (CARDIA)/Young Adult Longitudinal Trends in Antioxidants (YALTA) study. Clin Chem. 2007;53(3):447-55.

136. Hozawa A, Jacobs DR, Jr., Steffes MW, et al. Circulating carotenoid concentrations and incident hypertension: the Coronary Artery Risk Development in Young Adults (CARDIA) study. J Hypertens. 2009; 27(2):237-42. 
137. Rao AV, Rao LG. Carotenoids and human health. Pharmacol Res. 2007;55(3):207-16.

138. Duc le H, Fraser PD, Tam NK, Cutting SM. Carotenoids present in halotolerant Bacillus spore formers. FEMS Microbiol Lett. 2006;255(2):215-24.

139. Perez-Fons L, Steiger S, Khaneja R, et al. Identification and the developmental formation of carotenoid pigments in the yellow/orange Bacillus spore-formers. Biochim Biophys Acta. 2011 Mar; 181 1(3):177-85.

140. Khaneja R, Perez-Fons L, Fakhry S, et al. Carotenoids found in Bacillus. J Appl Microbiol. 2010; 108(6): 1889902. 


$$
8 \text { 国 }
$$




\section{chapter 2}

Randomised clinical study:

Aspergillus niger-derived enzyme digests gluten in the stomach of healthy volunteers

Salden BN, Monserrat V, Troost FJ, Bruins MJ, Edens L,

Bartholomé R, Haenen GR, Winkens B, Koning F, Masclee AA

Alimentary Pharmacology \& Therapeutics

2015 Aug;42(3):273-85 


\section{Abstract}

Background

Aspergillus niger prolyl endoprotease (AN-PEP) efficiently degrades gluten molecules into nonimmunogenic peptides in vitro.

\section{Aim}

To assess the efficacy of AN-PEP on gluten degradation in a low and high calorie meal in healthy subjects.

\section{Methods}

In this randomised, double-blind, placebo-controlled, cross-over study 12 healthy volunteers attended to four test days. A liquid low or high calorie meal $(4 \mathrm{~g}$ gluten) with AN-PEP or placebo was administered into the stomach. Via a triple-lumen catheter gastric and duodenal aspirates were sampled, and polyethylene glycol (PEG)-3350 was continuously infused. Acetaminophen in the meals tracked gastric emptying time. Gastric and duodenal samples were used to calculate 240-min area under the curve ( $\mathrm{AUC}_{0-240 \mathrm{~min}}$ ) of $\boldsymbol{\alpha}$-gliadin concentrations. Absolute $\alpha$-gliadin $\mathrm{AUC}_{0-240 \mathrm{~min}}$ was calculated using duodenal PEG-3350 concentrations.

Results

AN-PEP lowered $\boldsymbol{\alpha}$-gliadin concentration $\mathrm{AUC}_{0-240 \mathrm{~min}}$ compared to placebo, from low and high calorie meals in stomach (low: 35 vs. $389 \mu \mathrm{g}^{*} \mathrm{~min} / \mathrm{ml}$; high: 53 vs. $386 \mu \mathrm{g}^{*} \mathrm{~min} / \mathrm{ml} ; P<0.001$ ) and duodenum (low: 7 vs. $168 \mu g^{*} \mathrm{~min} / \mathrm{m}$.; high: 4 vs. $32 \mu \mathrm{g}^{*} \mathrm{~min} / \mathrm{ml} ; P<0.001$ ) and absolute $\alpha$-gliadin $\mathrm{AUC}_{0-240 \mathrm{~min}}$ in duodenum from low $\left(2,813\right.$ vs. 31,952 $\mu \mathrm{g}^{*}$ min; $\left.P<0.001\right)$ and high $\left(2,553\right.$ vs. $13,095 \mu \mathrm{g}^{*}$ min; $P=0.013)$ calorie meals. In the placebo group, high compared to low calorie meal slowed gastric emptying and lowered duodenal $\alpha$-gliadin concentration $A U C_{0-240 \min }\left(32 \mathrm{vs.} 168 \mu \mathrm{g}^{*} \mathrm{~min} / \mathrm{ml}\right.$; $P=0.0011$.

\section{Conclusions}

AN-PEP significantly enhanced gluten digestion in the stomach of healthy volunteers. Increasing caloric density prolonged gastric residence time of the meal. Since AN-PEP already degraded most gluten from low calorie meals, no incremental effect was observed by increasing meal caloric density. 


\section{Introduction}

Gluten is a storage protein present in wheat, barley and rye and is exceptionally rich in proline, rendering gluten peptides resistant to gastrointestinal digestion. Proline-cleaving proteases are absent in the human gastrointestinal tract. Therefore, long proline-rich gluten peptides reach the small intestine intact after ingestion. 'About $1 \%$ of the western population is suffering from celiac disease..$^{2-5}$ In these patients, exposure of duodenum and proximal small intestine to the specific amino acid sequences of such poorly digested proline-rich gluten peptides triggers an abnormal immune response. This causes inflammation with infiltration of lymphocytes in the intestinal mucosa and ultimately villous atrophy and crypt hyperplasia. ${ }^{6}$ Adverse reactions to gluten consumption are not limited to subjects suffering from celiac disease. Presently non-celiac gluten sensitivity has been clinically recognized as a separate condition in which neither allergic nor autoimmune mechanisms are involved. ${ }^{7.8}$ The symptoms experienced by these subjects are often identical to those seen in celiac disease. ${ }^{9.10}$

A lifelong gluten-free diet is the only treatment for individuals who cannot tolerate gluten. However, a gluten-free diet is hard to comply with as gluten-free products may not always be labelled, and may not always be at hand during social events or travelling. ${ }^{11-13}$

Prolyl endopeptidases belong to a family of enzymes with the ability to cleave at internal proline residues within a peptide. ${ }^{14}$ Early investigations on oral protease therapy as approach to degrade gluten have focused on bacterial prolyl oligopeptidases. ${ }^{15-18}$ However, several in vitro studies conducted with such enzymes revealed only low enzymatic activity at acidic stomach $\mathrm{pH}$ and rapid degradation of these enzymes by pepsin. ${ }^{16}$ Moreover, these enzymes were not able to prevent passage of potentially harmful gluten fragments into the small intestine. ${ }^{16,19}$ But, other enzymatic preparations have shown to be capable of degrading complex gluten proteins both in vitro and in vivo. ${ }^{20-23}$

In this respect, the Aspergillus niger-derived prolyl endoprotease (AN-PEP) also presents a promising option to degrade inadvertent dietary gluten. The use of the enzyme as food supplement has undergone successful evaluation by the French Agency for Food, Environmental and Occupational Health \& Safety. ${ }^{24}$ AN-PEP is active between $\mathrm{pH} 2$ and 6 , with optimum activity at gastric $\mathrm{pH}$ between 3 and $5 .{ }^{19}$ In a dynamic, multi-compartmental gastrointestinal in vitro model, AN-PEP was shown to degrade almost all immunogenic gluten epitopes from gluten-containing meals into non-immunogenic fragments during passage through the stomach compartment. ${ }^{25} \mathrm{~A}$ pilot study in celiac patients showed that a combination of AN-PEP and gluten was safe and well tolerated. ${ }^{26}$ Before AN-PEP can be used as a future digestive aid for subjects intolerant to gluten, it is essential that the promising in vitro results are confirmed in a human in vivo study, focussing on duodenal gluten delivery after intake of gluten-containing meals with and without AN-PEP. Thereafter the efficacy and safety of AN-PEP should be evaluated in target populations. 
Our aim was to investigate the efficacy of AN-PEP on gluten degradation in an intragastrically delivered gluten-containing meal in healthy volunteers. To standardize meal intake, we administrated the meal intragastrically at a fixed rate instead of by oral ingestion. Secondly, we hypothesized that increasing the caloric density of a meal enhances gluten degradation by delaying gastric emptying and thereby prolonging gastric residence time of the meal.

\section{Materials and methods}

The study was approved by the Medical Ethics Committee of the Maastricht University Medical Center (MUMC+) and conducted in full accordance with the principles of the Declaration of Helsinki of 1975 as revised in 2008 and with the Dutch Regulations on Medical Research involving Human Subjects (WMO, 1998). The study was performed at the MUMC+ from December 2011 to May 2012. All participants gave their written informed consent before participation. The trial has been registered in the Clinical Trials register (NCTO 1335503) and the Dutch trial register (NTR2780).

\section{Subjects}

Healthy volunteers aged 18-45 years were recruited by advertisement. All subjects were screened by means of a standardized general physical examination. Reasons for exclusion included: history of gastrointestinal disorders or gastrointestinal surgery interfering with gastrointestinal function; history of major disease, use of medication lexcept oral contraceptives) within 14 days before testing; dieting; pregnancy; lactation; excessive alcohol consumption (>20 alcoholic consumptions/week); and smoking.

\section{Design and intervention}

In this double-blind, randomized, placebo-controlled, crossover study, participants attended to four test days with at least 1 week washout period between two test days. At test days, participants were randomized in a double-blind fashion to 1 of the 24 possible orders of the four interventions; a low calorie gluten meal with AN-PEP or placebo, or a high calorie gluten meal with AN-PEP or placebo. The randomization list was generated by an independent and blinded statistician using a computerised procedure. All participants and investigators remained blinded to treatment until the analyses were completed. After an overnight fast, a triple-lumen catheter ladapted from Freka ${ }^{\oplus}$ Trelumina, Fresenius Kabi Nederland B.V., Zeist, The Netherlands) was introduced trans-nasally via the stomach into the duodenum, under fluoroscopic guidance. The proximal lumen, with an infusion port 
positioned in the stomach, was used for administration of the test meal and aspiration of gastric contents. The second lumen, positioned $5 \mathrm{~cm}$ distal to the pylorus, was used for continuous perfusion of the inert dilution marker polyethylene glycol (PEG)-3350. The third and distal lumen (positioned at tip and located $15 \mathrm{~cm}$ distal to the pylorus) was used for aspiration of duodenal contents. The catheter position was secured by radiology and regularly checked during the tests by measuring $\mathrm{pH}$ of each aspirate. Meals, mixed with acetaminophen (Centrafarm B.V., Etten-Leur, The Netherlands), and either AN-PEP or placebo, were infused into the stomach over a 5 -min period, at a rate of $80 \mathrm{ml} / \mathrm{min}$. A PEG3350 (Norgine B.V., Amsterdam, The Netherlands) solution $(15 \mathrm{mg} / \mathrm{ml}$ in $0.9 \%$ saline) was continuously infused into the proximal part of the duodenum at $3 \mathrm{ml} / \mathrm{min}$ to calculate the gluten amount corrected for dilution of duodenal content by endogenous secretions. ${ }^{27-30}$ Infusion started 60 min prior to meal infusion, to achieve steady state conditions in fluid secretion and absorption at the start of meal infusion, and continued until $240 \mathrm{~min}$. Gastric and duodenal content was sampled at baseline ( $t=0 \mathrm{~min}$ ), and after start of meal infusion at $t=15,30,45,60,75,90,120,150,180,210$ and 240 min. Gastric content was also aspirated at $t=5$ and $t=10$ min. Mixing of the meal with AN-PEP or placebo and acetaminophen was performed immediately prior to the start of infusion. Approximately $3 \mathrm{ml}$ and $2 \mathrm{ml}$ were aspirated from the gastric and duodenal port, respectively, for $\mathrm{pH}$ and gluten epitope measurements. Also, acetaminophen concentrations in gastric samples were measured. The samples were immediately frozen in liquid nitrogen to stop enzyme activity and were subsequently stored at $-80^{\circ} \mathrm{C}$ until analysis.

\section{Gluten meals}

Dry meals were packaged in sachets of airtight tinfoil and stored at room temperature. On test days, meals were prepared at a food-grade kitchen facility. During preparation of the test meal, $1 \mathrm{~g}$ acetaminophen was added. Table 2.1 shows the composition of the meals. All test meals contained $5.2 \mathrm{~g}$ of gluten powder, of which $4.0 \mathrm{~g}$ was gluten protein (Syral, Aalst, Belgium). Encapsulated refined olive oil powder (Vana Grasa 80B, FrieslandCampina, Kievit B.V., Meppel, The Netherlands) was used as fat source for the meals and together with maltodextrin as additional energy source for the high calorie meal, and sodium caseinate (DMV, Veghel, The Netherlands) was used to match both meals for protein content. The dry meal powders were dissolved in a total volume of $300 \mathrm{ml}$ tap water of $40^{\circ} \mathrm{C}$ by stirring with a spoon and subsequently mixing with a Turrax (IKA, Ultra Turrax ${ }^{\oplus}$ T25, Staufen, Germany). Low and high caloric meals were not blinded to the investigator. 
Table 2.1 Recipe and nutritional composition of the low and high calorie test meal per $300 \mathrm{~g}$ portion.

\begin{tabular}{lcc}
\hline & Low calorie meal & High calorie meal \\
\hline Vana Grasa (g) & 7.0 & 22.0 \\
Sucrose (g) & 17.0 & 16.9 \\
Maltodextrin (g) & 0.0 & 36.3 \\
Caseinate (g) & 0.7 & 0.3 \\
Gluten powder (g) & 5.2 & 5.2 \\
Citric acid (g) & 0.1 & 0.1 \\
Water (g) & 270.9 & 245.1 \\
Protein (g) & 4.9 & 4.9 \\
Fat (g) & 5.7 & 17.7 \\
Carbohydrate (g) & 18.0 & 56.5 \\
Ash (g) & 0.2 & 0.5 \\
Protein (kcal) & 19.6 & 19.6 \\
Fat (kcal) & 51.6 & 159.6 \\
Carbohydrate (kcal) & 71.9 & 225.8 \\
Total (kcal) & 143.1 & 405.1 \\
Caloric density (kcal/g) & 0.5 & 1.4 \\
Osmolarity (mOsm/kg) & 194.8 & 373.6 \\
pH & 6.0 & 6.0 \\
\hline
\end{tabular}

\section{AN-PEP enzyme and placebo}

The AN-PEP enzyme was obtained from DSM Food Specialties (Delft, The Netherlands). A total of $6.1 \mathrm{ml}$ of AN-PEP corresponding with 1.600.000 Protease Picomol International (1 Protease Picomol International is the amount of enzyme that releases one picomole of p-nitroaniline per second under defined assay conditions) in a total of $100 \mathrm{ml}$ water was added to the $300 \mathrm{~mL}$ test meals. A $6.1 \mathrm{ml}$ solution consisting of $4.8 \mathrm{~g}$ water, $1.3 \mathrm{~g}$ maltodextrin, $0.01 \mathrm{~g}$ caramel liquid (Brenntag, Deerlijk, Belgium), $0.03 \mathrm{~g}$ citric acid, and 0.02 g sodium benzoate (Wuhan Youji, Wuhan, China) at $\mathrm{pH} 4.2$, with a similar appearance to AN-PEP, served as placebo.

\section{Sample pre-treatment}

Upon thawing, the enzyme in the gastric and duodenal samples was immediately inactivated by increasing the $\mathrm{pH}$ of the sample to $11-12$ using $1 \mathrm{M} \mathrm{NaOH}$, heating at $85^{\circ} \mathrm{C}$ for 10 min and neutralizing the $\mathrm{pH}$ with $1 \mathrm{M} \mathrm{HCl}$. For the gluten content analysis, $100 \mu$ from each sample was frozen again at $-80^{\circ} \mathrm{C}$ for further analysis by ELISA, or mixed with loading buffer $160 \%$ glycerol, 300 mM Tris, pH 6.8, 12 mM EDTA, pH 8.0, 12\% sodium dodecylsulphate, $864 \mathrm{mM}$ 2-mercaptoethanol, 0.05\% bromophenol blue), boiled for $5 \mathrm{~min}$ for further analysis by Coomassie Blue staining and western blot. 


\section{Gluten monitoring by ELISA analyses}

The sample was diluted 40-5000 times in phosphate-buffered saline and the presence of the DO2.5-glia-a3 epitope was quantified using the Gluten-Tec ELISA assay (EuroProxima B.V., Arnhem, The Netherlands) according to the manufacturer's instructions. ${ }^{31}$ The DQ2.5glia-a3 epitope is directly adjacent to the 33-mer that contains the immunodominant DQ2.5-glia-a 1 and DQ2.5-glia-a 2 epitopes. As the $a$-gliadins contain only a single copy of the DQ2.5-glia-a3 epitope, the measurement of this epitope provides an accurate estimate of the actual $\boldsymbol{\alpha}$-gliadin content of the samples. ${ }^{32}$

\section{Volume marker}

PEG-3350 concentrations were determined in samples obtained from the distal aspiration port in the duodenum, using reversed-phase HPLC in combination with evaporative light scatter detection. The analysis was based on PEG analysis as described by van Wijck et al. ${ }^{33}$ PEG-3350 concentrations were used to calculate the dilution of duodenal samples by endogenous secretions, using the formula described by Beglinger et al. ${ }^{27}$

$V=\left(F \times[P E G]_{\text {perfused }} \times 15\right) /\left(P E G_{\text {measured }}\right)$

$\checkmark$ represents the calculated duodenal volume $(\mathrm{ml}$ per 15 or $30 \mathrm{~min})$; $F$ the flow rate of PEG solution perfused $(3 \mathrm{ml} / \mathrm{min})$; $[P E G]_{\text {perfused }}$ the concentration of PEG in the perfusate; PEG $G_{\text {measured }}$ the concentration of PEG in the duodenal juice collected for 15 or 30 min. The number ' 15 ' has to be replaced by '30' if the time interval between two samples was $30 \mathrm{~min}$.

To calculate the absolute duodenal gluten amount at a certain time point, the calculated duodenal volume was multiplied with the gluten concentration at that time point.

\section{Gluten monitoring by western blot}

Measurement of gluten epitopes by ELISA is an indirect method of gluten analysis. To confirm the presence of intact gluten proteins and relatively large fragments thereof we also performed Western blot analysis. The proteins present in samples isolated from the stomach were separated on SDS-PAGE, blotted onto polyvinylidenefluoride membrane and the gliadin proteins were visualized with a monoclonal antibody specific for the immunodominant DQ2.5-glia- 1 epitope. ${ }^{34}$ 


\section{Presence of AN-PEP in gastric and duodenal samples}

To assess the presence of AN-PEP protein in gastric and duodenal samples, the protein in the samples was separated on SDS-PAGE followed by Coomassie Blve staining.

\section{Assessment of gastric emptying}

Gastric emptying rate was measured at all test days of 6 of the 12 participants who completed the study, randomly chosen. Gastric emptying rate was determined according to the changes of the acetaminophen concentration over time in the stomach, with stomach samples taken at predetermined time points. Gastric fluid samples were first deproteinated by adding a 10\% solution of trichloroacetic acid. After centrifugation ( $800 \mathrm{~g}, 5 \mathrm{~min}$ ) the supernatant was injected into an HPLC using a reversed-phase method with UV detection at 250 nm (Agilent 1100 series HPLC Value System, Waldbronn, Germany). A composition of MilliO and acetonitrile (97: 3\%v/v) was used to elute the samples. As the total gastric volume changes constantly after meal ingestion, it was difficult to calculate the concentration via a formula. A calibration curve of acetaminophen was used to calculate the acetaminophen concentration in the samples. As a pragmatic approach, the total gastric emptying time (in minutes) was derived from this acetaminophen concentration-time curve. The time point when the acetaminophen concentration in a gastric sample is zero indicates the complete passage of the test meal into the duodenum and is thus considered to represent total gastric emptying time.

\section{Gastrointestinal symptoms questionnaire}

At the end of each test day, participants were requested to complete a 'Symptoms Diary', to ensure that all gastrointestinal complaints of the test day, caused by the intervention, were reported to the investigator. This questionnaire included 8 items, each rated on a fivepoint Likert scale. The lowest score, 1, denotes no symptoms and 5 denotes the most pronounced symptoms. Items that were included in the 'Symptoms Diary' were: abdominal discomfort, abdominal pain, abdominal distension, constipation, diarrhoea, flatus, eructation and nausea.

\section{Statistics}

The primary outcome of this study was the effect of AN-PEP on gluten degradation, measured by the difference in the 240-min AUC ( $\mathrm{AUC}_{0-240 \mathrm{~min}}$ ) of duodenal gluten epitope concentration between AN-PEP and placebo. Secondly, we investigated the effect of ANPEP on gluten degradation, measured by the difference in $\mathrm{AUC}_{0-240 \mathrm{~min}}$ of absolute amounts of gluten epitopes in the duodenum between both interventions. The $\mathrm{AUC}_{0-240 \mathrm{~min}}$ was 
calculated by using the trapezoidal equation. We calculated that a sample size of 12 subjects would be required based on a standardized effect-size of 1.3, a power of $90 \%$, and alpha $=0.05$ (one sided). Seventeen subjects were recruited taking into account a dropout of 5 subjects. Baseline characteristics are presented as mean (SD) for numerical variables and number (\%) for categorical variables. Differences in AUCs, gastric emptying rate and 'Symptom Diary' scores between combinations of treatment (AN-PEP or placebo) and meal (high or low calorie) were assessed using linear mixed models based on restricted maximum likelihood, where the natural logarithm of the AUCs was taken to account for the expected non-normality. The linear mixed model accounts for the correlation between repeated measures within a person (cross-over design) and missing data, where a likelihood approach was used assuming data missing at random. Fixed factors were treatment, meal, treatment*meal, and test-day. The best fitting covariance structure, i.e. structure of variances over different test-days and correlations between test-days, was based on Akaike's Information Criterion. All statistical analyses were performed using IBM SPSS Statistics for Windows (Version 20.0, IBM Corp, Armonk, NY, USA). Two-sided $P \leq 0.05$ were considered statistically significant.

\section{Results}

\section{Study subjects}

A total of 12 healthy volunteers $\left[67 \%\right.$ male; age $26 \pm 6$ years; $B M I\left(\right.$ in $\left.\left.\mathrm{kg} / \mathrm{m}^{2}\right)=22 \pm 3\right]$ were included in the study. One of these subjects did not complete the fourth test day and only the results of the two high calorie meal test days were available for inclusion in the analyses. In two other subjects the catheter progressed more distally into the small intestine at one occasion, causing administration of (part of) the meal directly into the duodenum. Data of these experiments were omitted, but data from their remaining test days were still included in the analysis as the linear mixed model accounts for missing data. Initially 17 subjects were enrolled in the study, but 5 subjects dropped out due to discomfort related to the nasoduodenal tube. Data of drop-outs were omitted from analyses.

\section{$\mathrm{pH}$ of gastric samples}

The mean gastric $\mathrm{pH}$ of gastric samples, taken during test days when low and high calorie meals combined with AN-PEP were infused, ranged between 2.3 and 5.3 and was similar to the $\mathrm{pH}$ range of the samples of placebo-containing meals (data not shown). 


\section{DQ2.5-glia- $\boldsymbol{\alpha} 3$ concentrations in stomach and duodenum and absolute}

\section{amount in duodenum}

The mean DO2.5-glia-a3 concentrations in stomach and duodenum samples after ingestion of low and high calorie meals with and without AN-PEP are shown in Figure 2.1 and the $\mathrm{AUC}_{0-240 \mathrm{~min}}$ in Table 2.2. The mean duodenal DO2.5-glia- $\alpha 3$ concentrations per participant are shown in Figure 2.2. Over a 240-min period, AN-PEP reduced the gastric DQ2.5-gliaa3 concentrations, compared to placebo, both after ingestion of the low (35 vs 389 $\left.\mu{ }^{*} \mathrm{~min} / \mathrm{ml} ; P<0.001\right)$ and the high $\left(53\right.$ vs $386 \mu \mathrm{g}^{*} \mathrm{~min} / \mathrm{ml} ; P<0.001$; Figure $2.1 \mathrm{~A}$ and $\mathrm{B}$; Table 2.2) calorie meals. This was also observed for duodenal DO2.5-glia-a3 concentrations (low calorie: 7 vs. $168 \mu \mathrm{g}^{*} \mathrm{~min} / \mathrm{ml}$; high calorie: 4 vs. $32 \mu \mathrm{g}^{*} \mathrm{~min} / \mathrm{ml}$; $P<0.001$; Figure 2.1C and D; Table 2.2). In the placebo intervention, the duodenal DO2.5-glia-a3 concentrations were significantly lower after intake of a high compared to a low calorie meal (32 vs. $168 \mu \mathrm{g}^{*} \mathrm{~min} / \mathrm{ml}$; $P=0.001$; Figure 2.1C and D; Table 2.2). In the presence of AN-PEP this difference was not present ( 4 vs. $7 \mu \mathrm{g}^{*} \mathrm{~min} / \mathrm{ml} ; P>0.05$; Figure $2.1 \mathrm{C}$ and $\mathrm{D}$; Table 2.2) and low duodenal DQ2.5-glia-a3 concentrations were observed after intake of both a high and low calorie meal. The $\mathrm{AUC}_{0-240 \mathrm{~min}}$ of DQ2.5-glia-a3 concentrations in the duodenum of AN-PEP-receiving subjects was around or lower than the limit of detection (26.7 $\mu \mathrm{g} / \mathrm{I}^{*} 240 \mathrm{~min}=7 \mu \mathrm{g} * \mathrm{~min} / \mathrm{ml}$ ) and lower than the limit of quantification $\left(89 \mu \mathrm{g} / \mathrm{l}^{*} 240\right.$ $\left.\min =21 \mu \mathrm{g}^{*} \min / \mathrm{l}\right)$ of the ELISA assay. The pattern for the duodenal DO2.5-glia-a3 concentrations corresponds with the data for absolute duodenal DO2.5-glia-a3 amount, which is corrected for the dilution during the digestion process. AN-PEP lowered the calculated absolute duodenal $\alpha$-gliadin compared to placebo in both low (2,813 vs. 31,952 $\mu \mathrm{g}^{*}$ min; $\left.P<0.001\right)$ and high (2,553 vs. 13,095 $\mu \mathrm{g}^{*}$ min; $P=0.013$; Figure 2.1E and F; Table 2.2) calorie meals.

\section{Gluten monitoring by western blot}

Western blot analysis of stomach samples indicated that gluten degradation was accelerated by the addition of AN-PEP. Compared to samples of placebo-containing meals, samples of AN-PEP-containing meals showed generally a markedly faster degradation of DQ2.5-glia-a 1. In many cases, very little or even no gluten protein could be detected when AN-PEP was taken with the meal. In contrast, in gastric samples of placebocontaining meals, in some cases DQ2.5-glia- 1 l was still detectable up to $2 \mathrm{~h}$ after meal infusion. In duodenal samples, the Western blots were unable to detect significant amounts of intact gluten proteins, neither with AN-PEP, nor with placebo (Figure 2.3). This finding 
suggests that in both cases little or no intact water insoluble gluten protein reaches the duodenum.

Overall, for the majority of the meals analysed, the pattern on gluten degradation by both ELISA and Western blot showed a correlation, demonstrating the robustness of the study results.
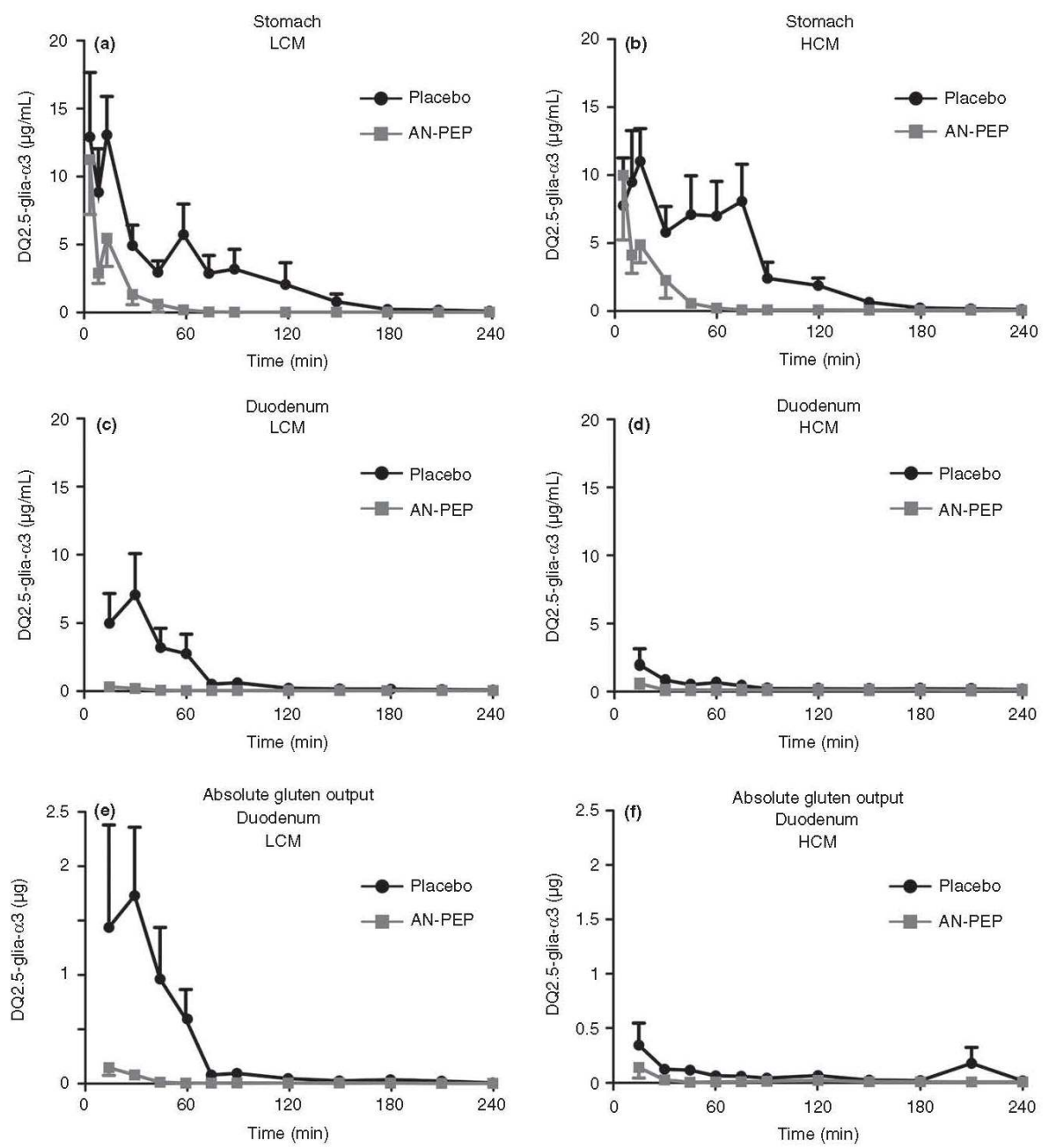

Figure 2.1 A: DQ2.5-glia-a3 concentration (mean \pm SEM) over time in the stomach in low calorie meals; B: DQ2.5-glia-a3 concentration (mean \pm SEM) over time in the stomach in high calorie meals; C: DQ2.5-glia-a3 concentration (mean \pm SEM) over time in the duodenum in low calorie meals; D: DO2.5-glia-a3 concentration (mean \pm SEM) over time in the duodenum in high calorie meals; E: Absolute DQ2.5-glia- $\alpha 3$ output (mean \pm SEM) over time in the duodenum in low calorie meals; F: Absolute DQ2.5-glia-a3 output (mean \pm SEM) over time in the duodenum in high calorie meals. 

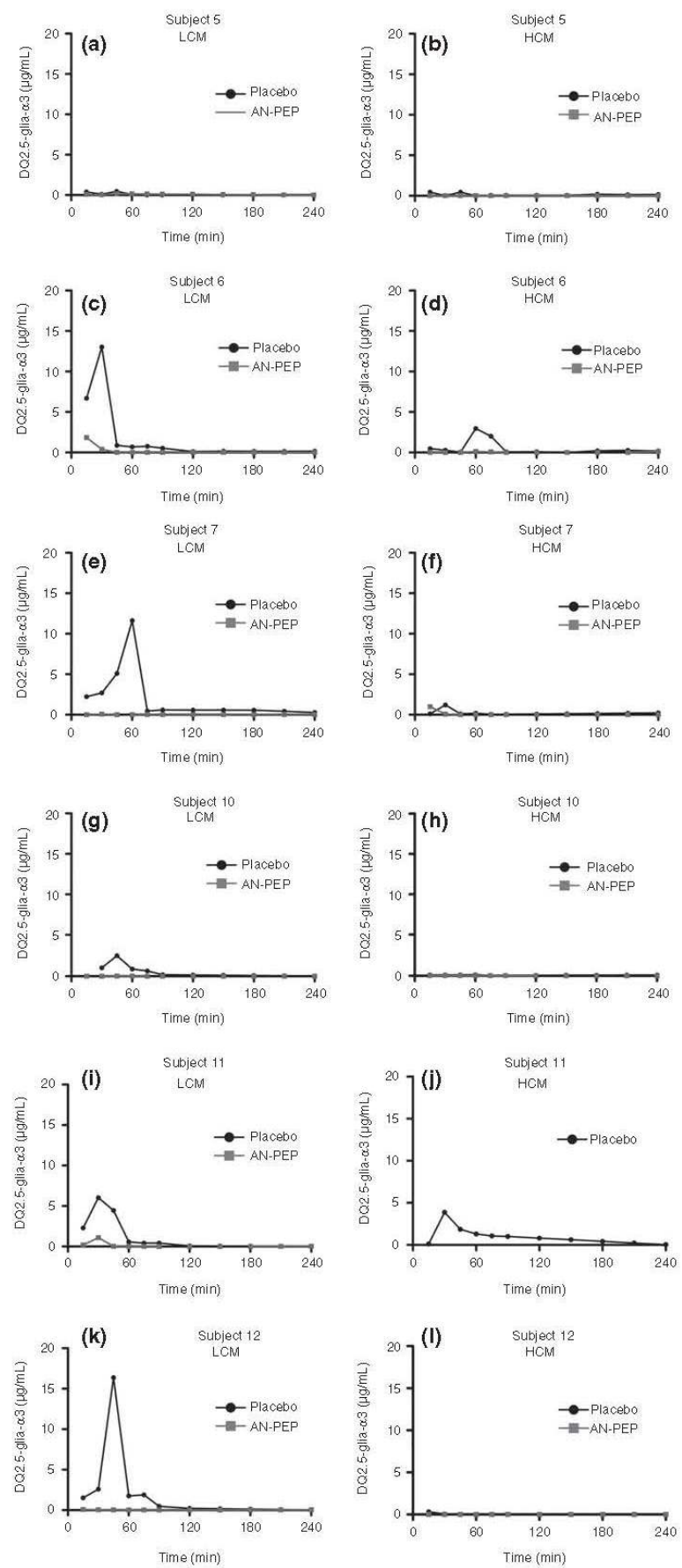

Figure 2.2 

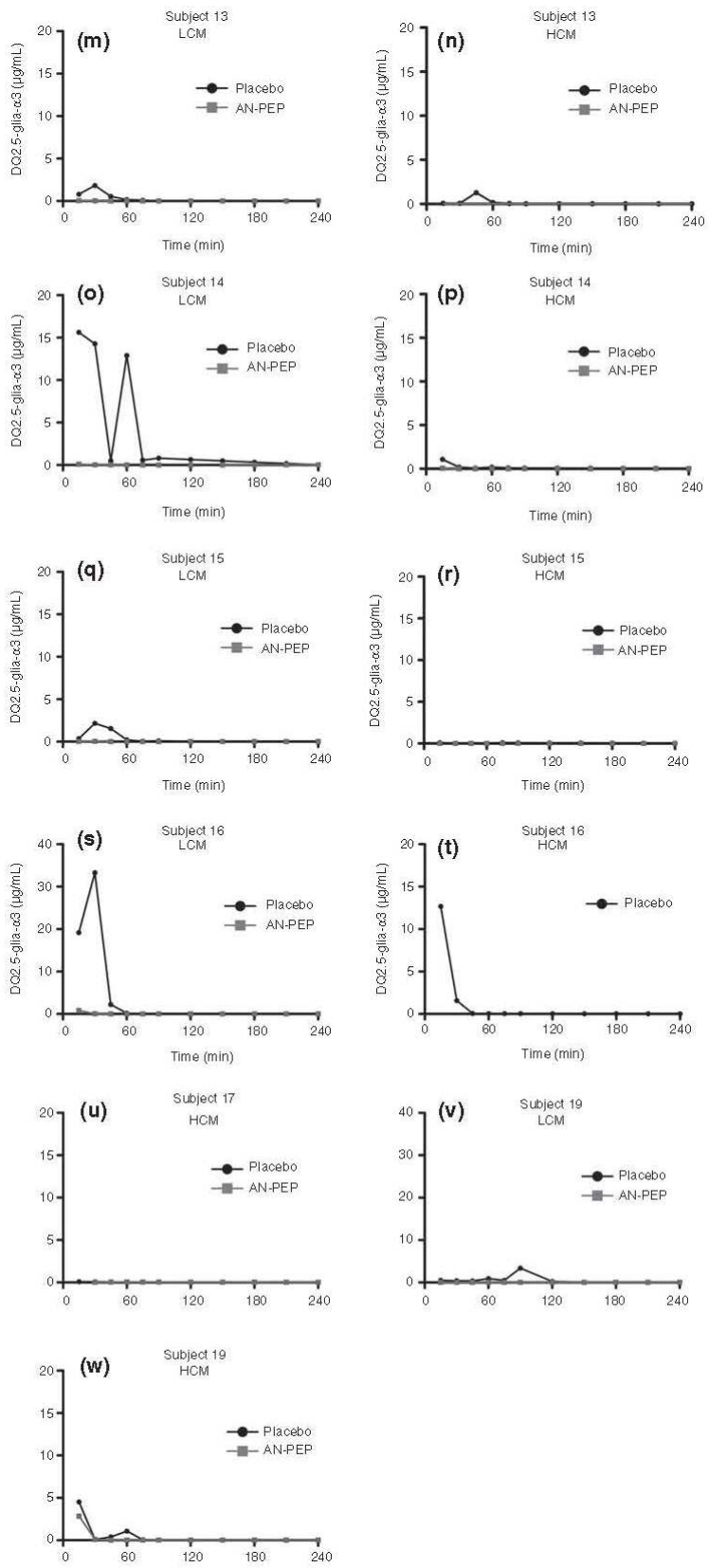

Figure 2.2 DQ2.5-glia- $\alpha 3$ concentration over time in the duodenum of the individual subjects in low calorie meals $(\mathrm{LCM})$ and high calorie meals $(\mathrm{HCM}) \cdot(\mathrm{a})+(\mathrm{b})$ : subject 5. (c) + (d): subject 6. (e) + (f): subject 7. $(\mathrm{g})+(\mathrm{h})$ : subject 10. (i) + (j): subject 11. (k) + (I): subject 12. $(\mathrm{m})+(\mathrm{n})$ : subject 13. (o) + (p): subject 14. (q) + (r): subject 15. $(\mathrm{s})+(t)$ : subject 16. (u): subject 17. $(\mathrm{v})+(\mathrm{w})$ : subject 19 . 
Table 2.2 AUC 0 -240min of DQ2.5-glia-a3 concentrations in stomach and duodenum and the $\mathrm{AUC}_{0-240 \mathrm{~min}}$ of absolute DQ2.5-glia-a3 amount in duodenum.

\begin{tabular}{|c|c|c|c|c|c|}
\hline & \multicolumn{2}{|c|}{ Low calorie meal } & \multicolumn{2}{|c|}{ High calorie meal } \\
\hline & & Placebo & AN-PEP & Placebo & AN-PEP \\
\hline \multicolumn{6}{|c|}{$\mathrm{AUC}_{0-240 \min } \mathrm{DQ} 2.5$-glia- $\alpha 3, \mu \mathrm{g}^{*} \mathrm{~min} / \mathrm{ml}$} \\
\hline \multirow[t]{2}{*}{ Stomach } & Mean & 389 & $35^{\circ}$ & 386 & $53^{a}$ \\
\hline & $95 \% \mathrm{Cl}$ & $180-840$ & $17-73$ & 192-775 & 25-113 \\
\hline \multirow[t]{2}{*}{ Duodenum } & Mean & 168 & $7^{a, d}$ & $32^{b}$ & $4^{a, e}$ \\
\hline & $95 \% \mathrm{Cl}$ & 80-352 & $3-14$ & $16-63$ & $2-9$ \\
\hline \multicolumn{6}{|c|}{$\mathrm{AUC}_{0-240 \min } \mathrm{DQ} 2.5$-glia- $\alpha 3, \mu \mathrm{g}^{*} \mathrm{~min} / \mathrm{ml}$} \\
\hline Duodenum & Mean & 31,952 & $2,813^{\circ}$ & 13,095 & $2,553^{c}$ \\
\hline & $95 \% \mathrm{Cl}$ & $12,670-80,579$ & $1,206-6,555$ & $5,967-28,730$ & $884-7,369$ \\
\hline
\end{tabular}

a $P<0.001$ compared to placebo; ${ }^{b} P=0.001$ compared to low calorie placebo meal; ${ }^{c} P<0.05$ compared to

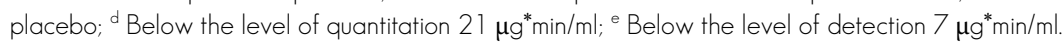

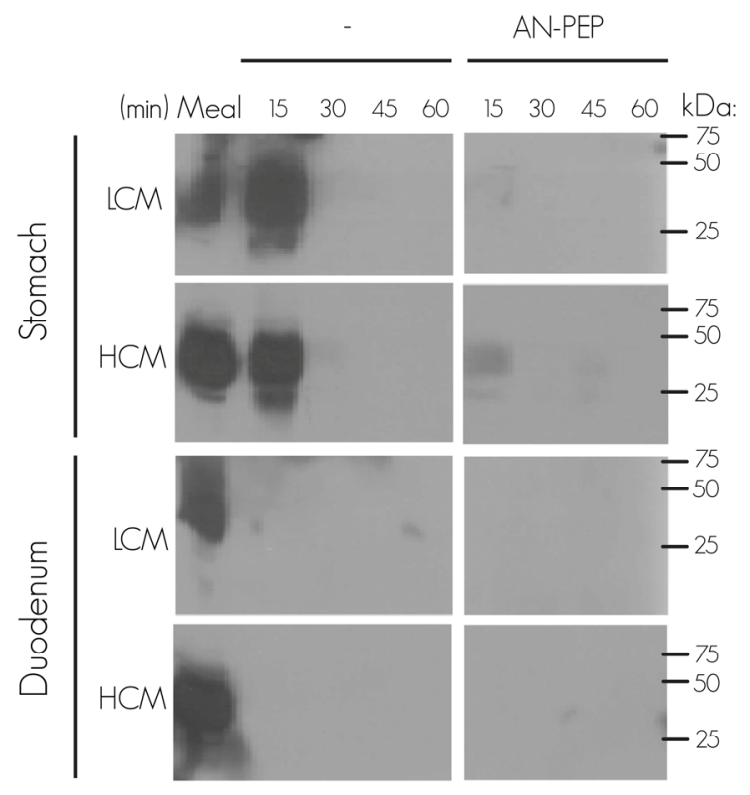

Figure 2.3 Representative Western blot showing degradation of water-insoluble DQ2.5-glia-a 1 over time in the stomach and duodenum in low and high calorie meals with and without AN-PEP.

\section{Presence of AN-PEP in gastric and duodenal samples}

After administration of AN-PEP-containing meals, a band with AN-PEP's characteristic molecular weight $(66 \mathrm{kD})$ was visible in the gastric samples of 14 different test days (Figure 2.4). In the other test days, the AN-PEP signal was either too weak to be detected or 
masked by other proteins with a similar electrophoretic mobility. In the placebo-containing meals, a band with this particular molecular weight was always absent (data not shown). With high calorie meals, AN-PEP was detectable for a longer period than with low calorie meals. AN-PEP was not found in the duodenal samples of any test day (Figure 2.4), possibly due to degradation of the enzyme by trypsin or chymotrypsin under conditions of high $\mathrm{pH}$ in the duodenum.

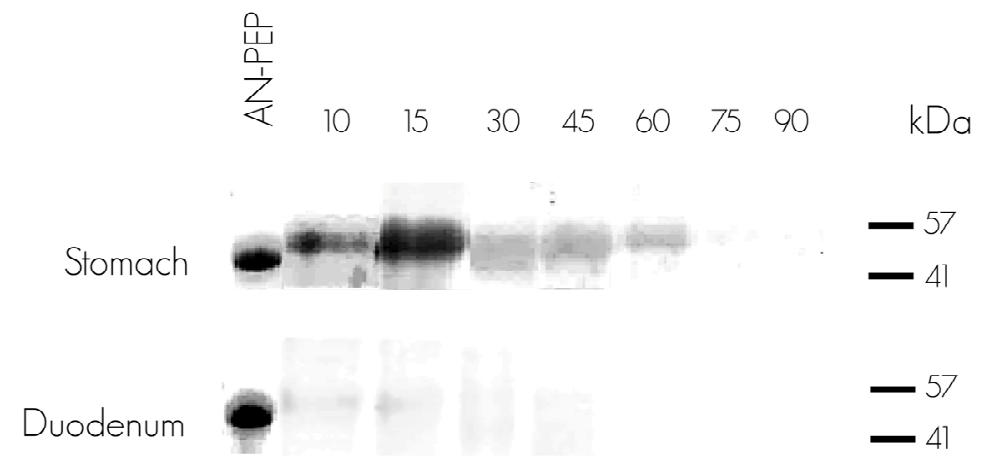

Figure 2.4 Representative SDS-PAGE gel showing the presence of AN-PEP protein in gastric and duodenal aspirates.

\section{Gastric emptying}

The mean of total gastric emptying time of the high calorie meals was approximately twice as long as compared to the low calorie meals, being significantly different in the presence of placebo (172 vs. $88 \mathrm{~min} ; P=0.014$ ) but not in the presence of AN-PEP (154 vs. $100 \mathrm{~min}$; $P=0.100)$.

\section{Gastrointestinal symptoms questionnaire}

Mild gastrointestinal symptoms were reported on some occasions during low and high calorie meal intervention. Overall, discomfort was low for each different intervention. No significant differences were observed in reported gastrointestinal symptoms between meal types in combination with AN-PEP or placebo (data not shown). 


\section{Discussion}

In the current placebo-controlled intervention study, AN-PEP-mediated gluten digestion was studied in the stomach and duodenum of healthy volunteers. This is the first study showing that the AN-PEP enzyme efficiently degrades gluten from a meal in the stomach of human subjects.

Considering the enzyme's optimum $\mathrm{pH}$ range between 3 and 5, and the mean $\mathrm{pH}$ of the gastric samples ranged between 2.3 and 5.3 with both low and high calorie meals, this points to optimal enzyme activity during the entire digestive process in the stomach. ${ }^{19}$ Irrespective of the caloric density of the meal, the enzyme degraded almost all gluten present in the stomach within a period of 1 hour whereas with placebo, gluten were present for 3 hours. Furthermore, the addition of AN-PEP did not result in differences in gastrointestinal-related symptoms compared to placebo, confirming that intake of the enzyme is safe and well tolerated by human subjects. ${ }^{26}$

We also tested whether increasing meal caloric density would improve gluten degradation by delaying gastric emptying and thus prolonging exposure time of gluten proteins to $\mathrm{AN}$ PEP and endogenous gastric proteases. Whereas the low calorie meal in the stomach was emptied within about 1.5 hour, the gastric emptying of the high calorie meal took about twice as long. Delayed gastric emptying also resulted in longer gastric residence time of AN-PEP. These findings are in line with a previously reported delay in gastric emptying rate with increased meal caloric density in human subjects. ${ }^{35}$ Within 1 hour, AN-PEP degraded gluten to concentrations around or below reliably detectable levels, irrespective of meal caloric density. Without AN-PEP enzyme, high gluten concentrations were present in the stomach when given with a low or high calorie meal, supporting the notion that pepsin exerts only minimal proteolytic action on dietary gluten. ${ }^{36}$ Interestingly, in absence of the enzyme, less gluten reached the duodenum with high than with low calorie meals. Possibly, the fat content of the high calorie meals supported gluten digestion in the duodenum by increasing pancreatic enzyme outputs which has been described for high-fat as compared to low-fat diets. ${ }^{37}$

We used a marker infusion technique to correct for dilutions resulting from biliary and pancreatic secretions that might have influenced the gluten concentrations measured in the duodenum. The absolute gluten values obtained, therefore, represent a better measure of true gluten exposure than the gluten concentrations. The absolute amount of gluten reaching the duodenum was significantly reduced with AN-PEP irrespective of meal caloric density, in line with the findings for gluten concentrations. The duodenal gluten degradation pattern was comparable between concentrations and absolute amounts suggesting little influence of duodenal dilutions. Insoluble gluten measurements confirmed that AN-PEP is able to significantly reduce gluten before entering the duodenum. 
A band on SDS-PAGE with AN-PEPs characteristic molecular weight was observed in gastric, but not in duodenal aspirates, indicating AN-PEP is present and active in the stomach but not in the duodenum. Possibly, under duodenal neutral $\mathrm{pH}$ conditions, bile and pancreatic enzymes may have degraded the enzyme.

Apart from AN-PEP, other enzymes detoxifying gluten are currently under investigation. A mixture of two proteases, namely PEP derived from Sphingomonas capsulate and a barley protease (EP-B2), termed ALVOO3 has previously been shown to be capable of degrading complex gluten proteins in vitro. ${ }^{20,23}$ In a human setting, ALVOO3 was well tolerated and effective in detoxifying $1 \mathrm{~g}$ of gluten. ${ }^{21}$ A recent study in celiac patients showed that ALVOO3 attenuated small intestinal mucosal injury induced by 6 weeks ingestion of $2 \mathrm{~g}$ gluten daily. ${ }^{22}$ Another protease mix, STAN-1, showed effective in vitro gluten-degrading properties. ${ }^{38}$ These enzymes have been investigated for their applicability as a future celiac disease drug therapy.

This study made use of a triple lumen nasogastroduodenal catheter. This enabled the simultaneous administration of a test meal, infusion of a dilution marker, and aspiration of gastric and duodenal contents. Clear benefit of this approach is that it allowed us to measure the actual gluten concentration present in duodenum samples. This information is important for safe use in subjects intolerant to gluten. Further, by infusion of the dilution marker we could calculate the absolute intraduodenal gluten appearance. In none of previous mentioned studies, investigating other gluten-detoxifying enzymes, true gluten presence in the duodenum has been measured. To standardize each meal intake, AN-PEP was added to the meal and thereafter immediately infused intragastrically at a standardized rate, to avoid differences in gluten degradation during meal consumption between interventions, caused by variable meal ingestion rates. We acknowledge that this does not represent a fully physiological meal setting, in which solid food and AN-PEP are ingested separately and undergo the normal physiologic processes of mixing in the stomach. A randomized placebo-controlled trial is underway in which the efficacy of AN-PEP in an actual meal setting will be investigated. Furthermore, this technique also has another drawback. Migration of the catheter, due to gastrointestinal peristalsis, caused erroneous infusion of the meal into the duodenum in two subjects at one occasion, as was noted based on $\mathrm{pH}$ profiles. These data were excluded from analysis.

The AN-PEP enzyme has been developed as a dietary supplement that in conjunction with a gluten-free diet may help subjects intolerant to gluten to digest unintended dietary gluten. Despite these promising results, the data do not prove that AN-PEP allows subjects intolerant to gluten to ingest gluten safely. Oral enzymes cannot replace a gluten-free diet, yet our observations suggest that AN-PEP may be useful as a digestive aid to help digest hidden gluten. The enzyme may protect against unintentional ingestion of gluten on a daily basis, during social events or travelling. A randomized placebo-controlled trial is underway 
to address AN-PEP's efficacy in the target population which is necessary prior to AN-PEP's use to be considered safe and effective.

In conclusion, AN-PEP efficiently degrades gluten from a meal in the stomach of healthy volunteers before entering the duodenum. Increasing the caloric density of a gluten meal slowed gastric emptying rate and prolonged gastric residence time of the enzyme. Since AN-PEP with a low calorie meal already degraded almost all gluten, a high calorie meal could not further increase the efficacy of the enzyme to digest gluten. 


\section{References}

1. Piper JL, Gray GM, Khosla C. Effect of prolyl endopeptidase on digestive-resistant gliadin peptides in vivo. J Pharmacol Exp Ther 2004;31 111):213-9.

2. West J, Logan RF, Hill PG, et al. Seroprevalence, correlates, and characteristics of undetected coeliac disease in England. Gut 2003;52(7):960-5.

3. Maki M, Mustalahti K, Kokkonen J, et al. Prevalence of Celiac disease among children in Finland. N Engl J Med 2003;348(25):2517-24.

4. Fasano A, Berti I, Gerarduzzi T, et al. Prevalence of celiac disease in at-risk and not-at-risk groups in the United States: a large multicenter study. Arch Intern Med 2003; 163(3):286-92.

5. Bingley PJ, Williams AJ, Norcross AJ, et al. Undiagnosed coeliac disease at age seven: population based prospective birth cohort study. BMJ 2004;328(7435):322-3.

6. Green PH, Cellier C. Celiac disease. N Engl J Med 2007:357(17): 1731-43.

7. Sapone A, Bai JC, Ciacci C, et al. Spectrum of gluten-related disorders: consensus on new nomenclature and classification. BMC Med 2012;10:13.

8. Sapone A, Lammers KM, Casolaro V, et al. Divergence of gut permeability and mucosal immune gene expression in two gluten-associated conditions: celiac disease and gluten sensitivity. BMC Med 201 1;9:23.

9. Sapone A, Lammers KM, Mazzarella G, et al. Differential mucosal IL-17 expression in two gliadin-induced disorders: gluten sensitivity and the autoimmune enteropathy celiac disease. Int Arch Allergy Immunol 2010;152(1):75-80.

10. Ferguson A, Gillett H, Humphreys K, Kingstone K. Heterogeneity of celiac disease: clinical, pathological, immunological, and genetic. Ann N Y Acad Sci 1998:859:1 12-20.

11. Lerner A. New therapeutic strategies for celiac disease. Autoimmun Rev 2010;9(3): 144-7.

12. Sollid LM, Khosla C. Future therapeutic options for celiac disease. Nat Clin Pract Gastroenterol Hepatol 2005;2(3): 1 140-7.

13. Sollid LM, Khosla C. Novel therapies for coeliac disease. J Intern Med 201 1;269(6):604-13.

14. Gass J, Khosla C. Prolyl endopeptidases. Cell Mol Life Sci 2007;64(3):345-55.

15. Matysiak-Budnik T, Candalh C, Cellier C, et al. Limited efficiency of prolyl-endopeptidase in the detoxification of gliadin peptides in celiac disease. Gastroenterology 2005; 129(3):786-96.

16. Shan L, Marti T, Sollid LM, Gray et al. Comparative biochemical analysis of three bacterial prolyl endopeptidases: implications for coeliac sprue. Biochem J 2004;383(P+ 2):31 1-8.

17. Shan L, Molberg O, Parrot I, et al. Structural basis for gluten intolerance in celiac sprue. Science 2002;297(5590):2275-9.

18. Gass J, Ehren J, Strohmeier $G$, et al. Fermentation, purification, formulation, and pharmacological evaluation of a prolyl endopeptidase from Myxococcus xanthus: implications for Celiac Sprue therapy. Biotechnol Bioeng 2005;92(6):674-84.

19. Stepniak D, Spaenii-Dekking L, Mitea C, et al. Highly efficient gluten degradation with a newly identified prolyl endoprotease: implications for celiac disease. Am J Physiol Gastrointest Liver Physiol 2006;291(4):G621-9.

20. Gass J, Vora H, Bethune MT, et al. Effect of barley endoprotease EP-B2 on gluten digestion in the intact rat. J Pharmacol Exp Ther 2006;318(3): 1 178-86.

21. Siegel M, Garber ME, Spencer AG, et al. Safety, tolerability, and activity of ALVOO3: results from two phase 1 single, escalating-dose clinical trials. Dig Dis Sci 2012;57(2):440-50.

22. Lahdeaho ML, Kaukinen K, Laurila K, et al. Glutenase ALVOO3 attenuates gluten-induced mucosal injury in patients with celiac disease. Gastroenterology 2014; 146(7): 1649-58.

23. Gass J, Bethune MT, Siegel M, et al. Combination enzyme therapy for gastric digestion of dietary gluten in patients with celiac sprue. Gastroenterology 2007;133(2):472-80 
24. ANSES - French Agency for Food EaOHS. AVIS de l'Anses relatif à une demande d'autorisation de mise sur le marché d'un nouvel aliment ou d'un ingrédient alimentaire: préparation enzymatique contenant une activité protéase pour une utilisation dans les compléments alimentaires. In; 2014.

25. Mitea C, Havenaar R, Drijfhout JW, et al. Efficient degradation of gluten by a prolyl endoprotease in a gastrointestinal model: implications for coeliac disease. Gut 2008;57(1):25-32.

26. Tack GJ, van de Water JM, Bruins MJ, et al. Consumption of gluten with gluten-degrading enzyme by celiac patients: a pilot-study. World J Gastroenterol 2013; 19(35):5837-47.

27. Beglinger $C$, Fried $M$, Whitehouse I, et al. Pancreatic enzyme response to a liquid meal and to hormonal stimulation. Correlation with plasma secretin and cholecystokinin levels. J Clin Invest 1985;75(5): 1471-6.

28. Vu MK, van der Veek PP, Frolich M, et al. Does jejunal feeding activate exocrine pancreatic secretion? Eur J Clin Invest 1999;29(12): 1053-9.

29. Lam WF, Gielkens HA, Coenraad M, et al. Effect of insulin and glucose on basal and cholecystokininstimulated exocrine pancreatic secretion in humans. Pancreas 1999; 18(3):252-8.

30. Symersky T, Vu MK, Frolich M, et al. The effect of equicaloric medium-chain and long-chain triglycerides on pancreas enzyme secretion. Clin Physiol Funct Imaging 2002;22(5):307-1 1.

31. Mujico JR, Dekking L, Kooy-Winkelaar Y, et al. Validation of a new enzyme-linked immunosorbent assay to detect the triggering proteins and peptides for celiac disease: interlaboratory study. J AOAC Int 2012;95(1):206-15.

32. Sollid LM, Qiao SW, Anderson RP, et al. Nomenclature and listing of celiac disease relevant gluten T-cell epitopes restricted by HLA-DQ molecules. Immunogenetics 2012;64(6):455-60.

33. van Wijck K, Bessems BA, van Eijk HM, Buurman WA, Dejong CH, Lenaerts K. Polyethylene glycol versus dual sugar assay for gastrointestinal permeability analysis: is it time to choose? Clin Exp Gastroenterol 2012;5: 139-50.

34. Spaenii-Dekking EH, Kooy-Winkelaar EM, Nieuwenhuizen WF, et al. A novel and sensitive method for the detection of T cell stimulatory epitopes of alpha/beta- and gamma-gliadin. Gut 2004;53(9): 1267-73.

35. Kwiatek MA, Menne D, Steingoetter A, et al. Effect of meal volume and calorie load on postprandial gastric function and emptying: studies under physiological conditions by combined fiber-optic pressure measurement and MRI. American Journal of Physiology - Gastrointestinal and Liver Physiology 2009;297(5):G894-G901.

36. Hausch F, Shan L, Santiago NA, et al. Intestinal digestive resistance of immunodominant gliadin peptides. Am J Physiol Gastrointest Liver Physiol 2002;283(4):G996-G1003.

37. Boivin M, Lanspa SJ, Zinsmeister AR, et al. Are diets associated with different rates of human interdigestive and postprandial pancreatic enzyme secretion? Gastroenterology 1990;99(6): 1763-71.

38. Lahdeaho ML, Lindfors K, Airaksinen L, et al. Recent advances in the development of new treatments for celiac disease. Expert Opin Biol Ther 2012;12(12):1589-600. 

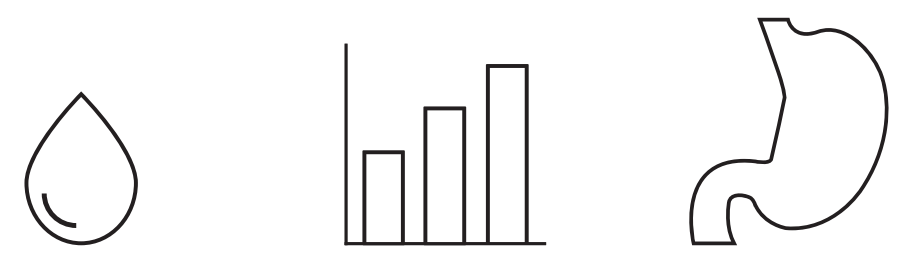


\section{chapter}

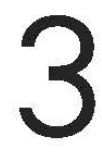

Paracetamol as a post prandial marker for gastric emptying, a food-drug interaction on absorption

Bartholomé R, Salden BN, Vrolijk MF, Troost FJ, Masclee AA, Bast A, Haenen GR

PloS One 2015; 10:e0136678 


\section{Abstract}

The use of paracetamol as tool to determine gastric emptying was evaluated in a cross-over study. Twelve healthy volunteers were included and each of them consumed two low and two high caloric meals. Paracetamol was mixed with a liquid meal and administered by a nasogastric feeding tube. The post prandial paracetamol plasma concentration time curve in all participants and the paracetamol concentration in the stomach content in six participants were determined. It was found that after paracetamol has left the stomach, based on analysis of the stomach content, there was still a substantial rise in the plasma paracetamol concentration time curve. Moreover, the difference in gastric emptying between high and low caloric meals was missed using the plasma paracetamol concentration time curve. The latter curves indicate that (i) part of the paracetamol may leave the stomach much quicker than the meal and (ii) part of the paracetamol may be relatively slowly absorbed in the duodenum. This can be explained by the partition of the homogenous paracetamolmeal mixture in the stomach in an aqueous phase and a solid bolus. The aqueous phase leaves the stomach quickly and the paracetamol in this phase is quickly absorbed in the duodenum, giving rise to the relatively steep increase of the paracetamol concentration in the plasma. The bolus leaves the stomach relatively slowly, and encapsulation by the bolus results in relatively slow uptake of paracetamol from the bolus in the duodenum.

These findings implicate that paracetamol is not an accurate post prandial marker for gastric emptying. The paracetamol concentration time curve rather illustrates the food-drug interaction on absorption, which is not only governed by gastric emptying. 


\section{Introduction}

In order to display a systemic biological effect most compounds first have to be absorbed from the gastro-intestinal tract. The onset of the effect depends heavily on the rate of absorption. Absorption mainly takes place once the compound has reached the intestine. Gastric emptying is considered to be the bottleneck in uptake, and numerous studies have been conducted on the effect of nutrition and disease on this process.

Paracetamol is the number one tool for monitoring gastric emptying.' To assess gastric emptying, paracetamol is mixed through a meal. After ingestion, the meal containing paracetamol will enter the stomach, and the meal is digested. Gradually the mealparacetamol mixture passes through the pyloric sphincter into the duodenum. The large surface area, the peristaltic movement, the relatively thin membrane and the rich blood supply of the intestine enable rapid uptake in the duodenum. It is assumed that paracetamol will be absorbed almost immediately after leaving the stomach. Moreover, it is assumed that the passage time of paracetamol through the stomach is identical to that of the meal. Based on these assumptions, the rate of paracetamol uptake into the plasma would be governed by gastric emptying and consequently gastric emptying might be deduced from the time course of the plasma concentration of paracetamol. ${ }^{2-5}$

This approach with paracetamol as marker molecule is regarded as an established and well validated method and is extensively applied. The wide application and acceptance of the procedure, might explain why a critical attitude is lacking. Often, only a small number of samples (sometimes even only one) is taken which will give only a rudimentary kinetic profile. Surprisingly, only a few studies addressed the validity of the method. ${ }^{6,7}$ In the most extensive literature review on the subject is was stated that "it is a simple, noninvasive, and economical method, making it suitable for application on a larger scale but further research should be performed under standardized conditions to allow wide scale clinical use". Moreover, in a recent study, we obtained data which are in conflict with the widely accepted model which prompted us to carefully evaluate the use of paracetamol as post prandial marker for gastric emptying. This revealed two major pitfalls, i.e. (i) part of the paracetamol may leave the stomach much quicker than the meal and (ii) part of the paracetamol may be relatively slowly absorbed after entering the duodenum.

\section{Materials and methods}

\section{Clinical study}

In the randomized cross-over study "Aspergillus niger-derived enzyme effectively digests gluten in the stomach of healthy volunteers"8 (December 2011 to May 2012) gastric 
emptying was assessed with the use of paracetamol. The healthy volunteers attended four test days with at least one week washout period between two test days. On two test days the volunteers were given a low caloric meal (143 kcal), administered via a nasogastric feeding tube (Freka ${ }^{\oplus}$ Trelumina, Fresenius Kabi Nederland b.v., Zeist, The Netherlands). One of the two low caloric meals given to each of the volunteers was mixed with Aspergillus niger-derived prolyl endoprotease (AN-PEP). On the other two test days a high caloric meal (405 kcal) was administered. Also one of the two high caloric meals given to each of the volunteers was mixed with AN-PEP. The AN-PEP treatment had no effect on the paracetamol data and therefore the results of the meal treated with AN-PEP were combined with that of the meal that was not treated.

Prior to administration, $1000 \mathrm{mg}$ paracetamol was added to the meals and the meal was thoroughly mixed. The rate of gastric emptying was determined using two different strategies. The common strategy is based on the paracetamol plasma concentration. Blood samples were taken 0, 10, 15, 30, 60, 90, 120, 150, 180, 210 and 240 minutes after administration of the meal. Further details regarding materials and methods are described earlier by Salden et al.. ${ }^{8}$ The flow diagram of the study is presented in Figure 3.1.

A total of 12 ( 8 male and 4 female) healthy volunteers (average age $26 \pm 6$ years, average BMI $22 \pm 3 \mathrm{~kg} / \mathrm{m}^{2}$ ) were included in the study. One of these subjects did not complete the fourth test day. In two other subjects the catheter progressed more distally into the small intestine on one occasion (based on the high pH of the sample), indicating that (part of) the meal was directly administered into the duodenum. These experiments were omitted, but the other experiments of these subjects were included in the data analysis. Initially 17 subjects were enrolled in the study, but 5 subjects dropped out due to discomfort related to the nasoduodenal tube. Data of dropouts were omitted from analyses.

The amount of paracetamol taken up on 10 and 240 minutes after intake was derived from the area under concentration-time curve in all subjects. The second strategy to determine gastric emptying was based on the paracetamol concentration in the content of the stomach. Samples were aspirated from the stomach on 15, 30, 45, 60, 75, 90, 120, 150, 180, 210 and 240 minutes after intake. In 6 subjects a complete set of stomach samples could be obtained, and the paracetamol content of the stomach samples of these subjects was determined. Paracetamol metabolites were not determined, because the aim of the study was to evaluate the use of paracetamol as tool for gastric emptying. The paracetamol concentration-time curve derived from the HPLC analysis of the sample was used to determine gastric emptying.

The fractionation of paracetamol in the stomach was mimicked by adding $100 \mu \mathrm{HCl}$ solution $(0.01 \mathrm{M})$ to $400 \mu \mathrm{l}$ of the meal to obtain a $\mathrm{pH}$ of 2 . After centrifugation the pellet was resuspended in a sodium-phosphate buffer of $\mathrm{pH} 7.4$ and centrifuged again. In both supernatants the paracetamol concentration was determined using HPLC. 
The study has been approved by the Institutional Review Board of Maastricht University, and all clinical investigations have been conducted according to the principles expressed in the Declaration of Helsinki. Written informed consent has been obtained from the participants. The study has been registered as NTR2780 in the Dutch trialregister (Nederlands Trialregister).

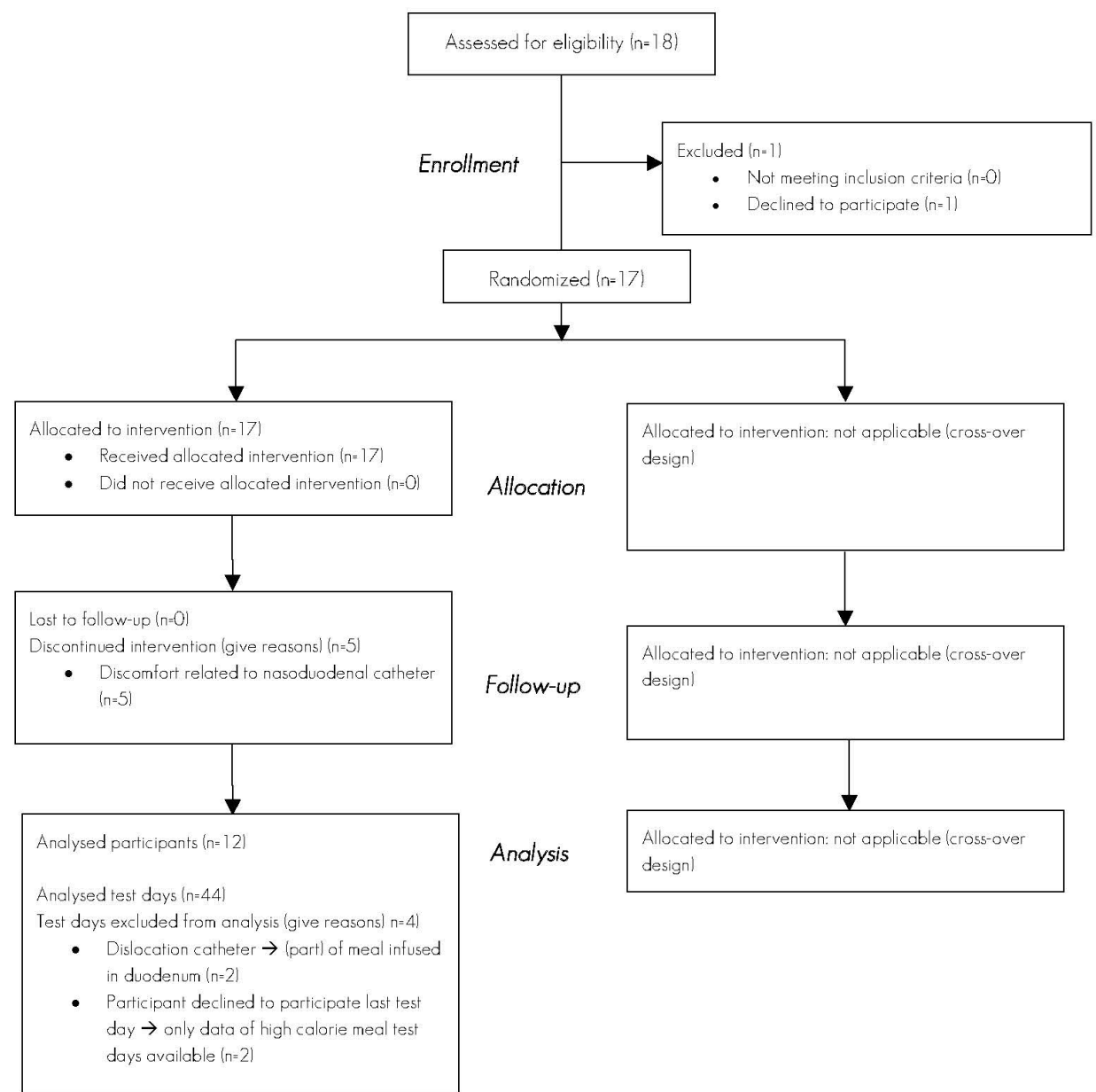

Figure 3.1 Consort Flow Diagram of the study.

\section{Determination of paracetamol}

For the determination of the paracetamol concentration, the samples were deproteinated by adding trichloroacetic acid. After centrifugation ( $800 \times g, 5$ minutes) the supernatant was 
analyzed using reversed phase HPLC with UV detection at $250 \mathrm{~nm}$. The eluent was a mixture of Milli@ and Acetonitrile (97: $3 \% \mathrm{v} / \mathrm{v})$. The quality control samples as well as the calibration curves were well within the specifications for pharmacokinetic studies. ${ }^{10}$ For each subject and each treatment, an extra control sample taken before the administration of Paracetamol was taken. In none of these control samples interfering peaks were observed. The analyses were performed blinded to the study.

\section{Statistics}

The concentration time curve was statistically evaluated in Prism 6 (GraphPad Software, La Jolla, USA). Differences in pharmacokinetic parameters between groups were analyzed using ANOVA. Post-hoc testing was performed using a paired t-test with Bonferroni correction. $A P<0.05$ was considered to be statistically significant.

\section{Results}

A liquid meal was administered as a slurry by a nasogastric feeding tube. Paracetamol was mixed through the meal prior to administration for monitoring gastric emptying. Subsequently, the concentration of paracetamol in plasma was determined post prandial. In six volunteers, also the concentration of paracetamol in the content of the stomach was determined.

\section{Partition of the paracetamol}

The paracetamol level in plasma rose after administration of the meal, until it reached a maximum concentration $\left(\mathrm{C}_{\max }\right)$ relatively quickly with the low caloric meals. After reaching $\mathrm{C}_{\text {max }}$, the concentration time curve of paracetamol in plasma showed a biphasic exponential decay. Surprisingly, the exponential decay immediately after reaching $C_{\max }$ was higher compared to that at a later stage $(P<0.05)$. An illustration of this kinetic profile is given in Figure 3.2A.

From the plasma paracetamol data the amount of paracetamol which was taken up was calculated. It appeared that in most subjects, already within 15 minutes, an unexpected high amount of paracetamol was absorbed, even up to $70 \%$ of the total dose (Figure 3.2). The uptake in two phases indicates that paracetamol, administered mixed with the meal, is divided into two fractions. One fraction enters quickly the duodenum and is responsible for the fast uptake. The other fraction apparently stays relatively long in the stomach and is responsible for the relatively slow uptake. 

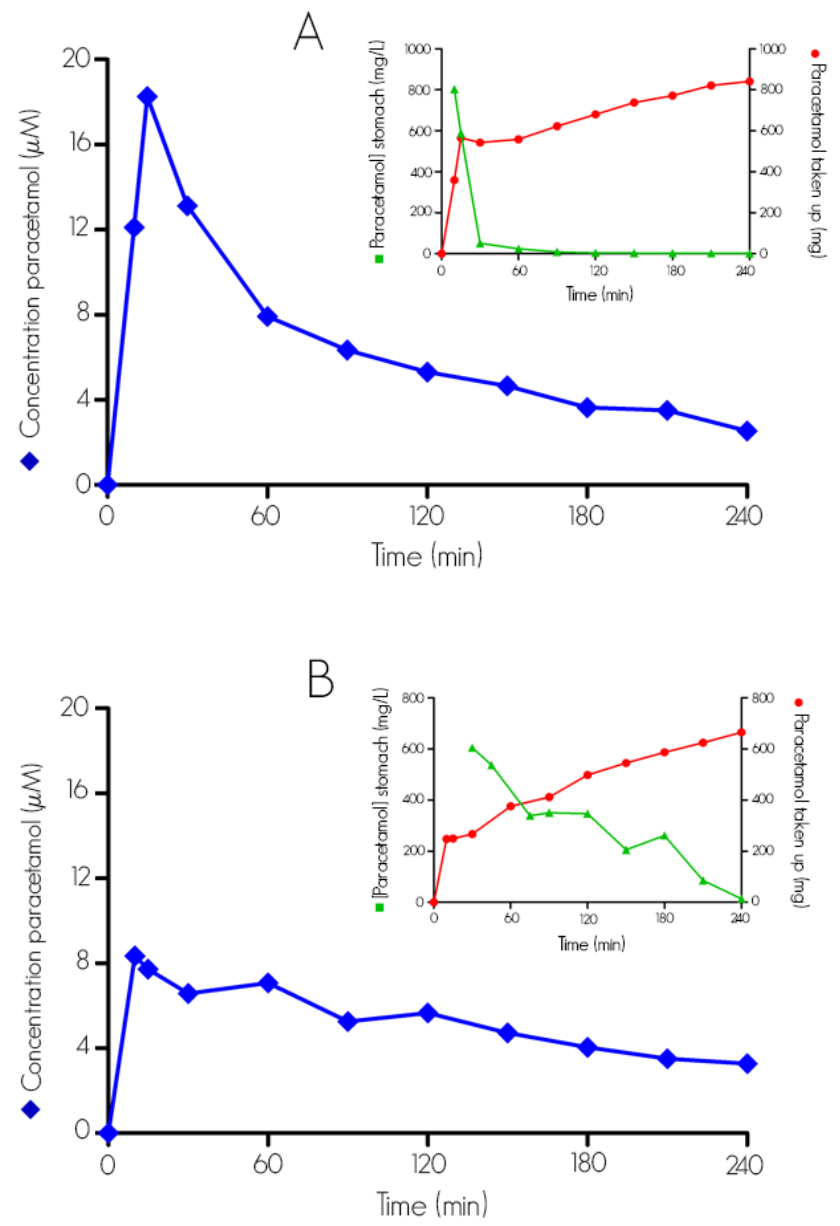

Figure 3.2 The plasma paracetamol concentration in time after administration of a low or high caloric meal containing $1 \mathrm{~g}$ paracetamol via a nasogastric tube. (-) (Panel A, low caloric meal; panel B, high caloric meal). A typical example is shown. The inserts show the uptake of paracetamol based on the plasma concentration curve ( ) and the concentration of paracetamol detected in the stomach content ( ) (panel A, low caloric meal; panel B, high caloric meal).

\section{Encapsulation of part of the paracetamol by the meal}

To further investigate the fractionation of paracetamol, a hydrochloric acid solution (to obtain a pH of 2) was added to the low caloric meal containing paracetamol in a test tube to mimic the condition in the stomach. The obtained mixture was centrifuged and two thirds of the paracetamol was retrieved in the supernatant. The pellet was mixed with buffer (150 mM sodium phosphate buffer, $\mathrm{pH} 7.4$ ) and centrifuged again. This extract of the pellet 
appeared to comprise approximately one tenth of the original amount of paracetamol (Figure 3.3). Similar results were obtained with the high caloric meal instead of the low caloric meal (data not shown). These experiments substantiate that in the stomach the paracetamol is divided into two fractions, which are (i) a fraction in which paracetamol is dissolved in the liquid content of the stomach and (ii) a fraction in which paracetamol is encapsulated by the contents of the meal that precipitate in the acid environment of the stomach.

The conclusion is corroborated when the plasma concentration in time is compared to the level of paracetamol detected in the content of the stomach. A slow, but substantial paracetamol uptake in the plasma occurs even when the paracetamol has left the stomach, as shown in Figure 3.2. This indicates that slow release in the intestine of the paracetamol that is encapsulated by a meal, is a rate-limiting process in the uptake.

\section{Paracetamol distribution}

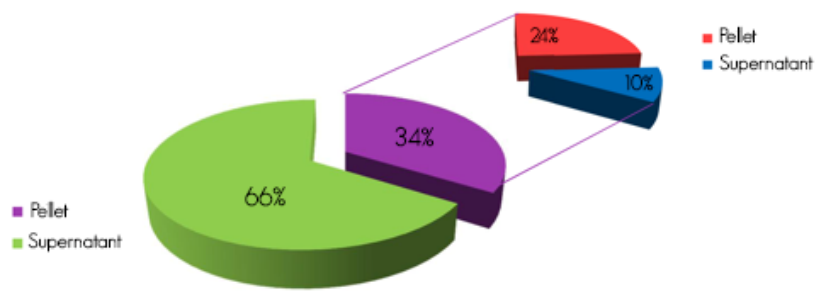

Figure 3.3 Encapsulation of paracetamol by the meal. The meal containing paracetamol was mixed with a hydrochloric acid solution $(\mathrm{pH} 2)$ in a test tube to mimic the conditions in the stomach. After centrifugation two thirds of the paracetamol was retrieved in the supernatant. Extraction of the pellet with buffer $(150 \mathrm{mM}$ sodium phosphate buffer, $\mathrm{pH} 7.4)$ released one tenth of the paracetamol.

\section{High and low caloric meals}

In our study, high and low caloric meals were given to the volunteers. The rate of gastric emptying of these meals was estimated from the plasma paracetamol concentration and the concentration of the first time point (10 minutes) was used for this. After 10 minutes a substantial amount of paracetamol, on average 35\%, was taken up. No difference was found in the 10 minutes paracetamol concentrations between the low and high caloric meal, suggesting that gastric emptying did not differ between both types of meal (Figure 3.4A). Also after 240 minutes, the plasma paracetamol data indicate that there is no difference in gastric emptying between the low and high caloric meals (Figure 3.4B). 
The time at which no paracetamol was detected in the stomach content is a direct measure for gastric emptying. These stomach data show that gastric emptying was statistically longer with the high caloric meal compared to the low caloric meal (Figure 3.4C). The lower gastric emptying of a high caloric meal compared to that of a low caloric meal is well documented, and this difference is confirmed by the longer retention of paracetamol in the stomach samples after the high caloric meal compared to that after the low caloric meal. The difference between the low and high caloric meal is not observed in the gastric emptying determined using the plasma paracetamol concentration (Figure 3.4). ${ }^{11}$

\section{Plasma data}

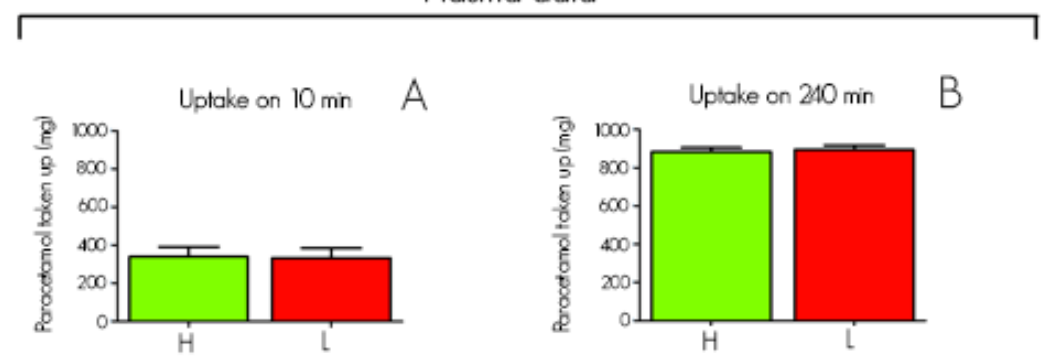

Stomach data

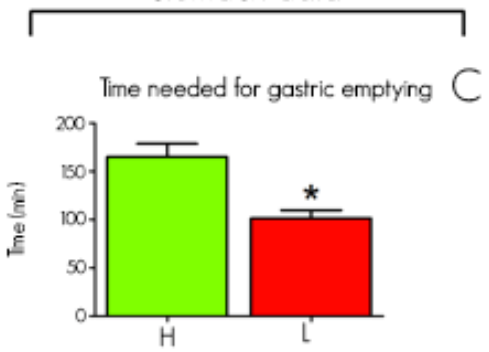

Figure 3.4 Discrepancy in gastric emptying deduced trom the paracetamol plasma data and deduced from content in the stomach. The amount of paracetamol taken up after $10 \mathrm{~min}$ (panel A) and $240 \mathrm{~min}$ (panel B) calculated form the concentration time curve of paracetamol in plasma do not show a difference between the high and low caloric meals. However, the time needed for gastric emptying determinded by the paracetamol content of the stomac (panel C), show a clear difference in gastric emptying between the high and low caloric meals. * different form the high caloric value $(P<0.05)$.

\section{Discussion}

In the present study, the use of paracetamol as tool for gastric emptying is evaluated. The nasogastric feeding tube which was used in our experimental set up, assured that the complete and homogeneous meal/paracetamol mixture was in the stomach at the start of 
the experiment. After administering the meal, a relatively steep initial increase in the plasma concentration of paracetamol was seen, indicative for a relatively quick uptake. After the quick initial rise, the plasma paracetamol concentration demonstrated a more prolonged, gradual uptake of paracetamol in the blood. Even when all paracetamol has left the stomach, based on analysis of the stomach content, there was still a slow but substantial rise uptake with all meals and in all volunteers. That there still is a substantial uptake of paracetamol when all of the paracetamol has left the stomach, already indicates that the plasma paracetamol levels do not only reflect gastric emptying.

The biphasic time course of the paracetamol curve can be explained by the division of paracetamol into two fractions after entering the stomach, a fraction that is encapsulated by the meal and a fraction that is dissolved in the liquid content of the stomach. The aqueous phase is expected to leave the stomach quickly and paracetamol in an aqueous phase is readily absorbed in the duodenum. Deduced from the relatively high and early peak in the plasma paracetamol time course, most paracetamol is in this aqueous phase. This is corroborated by the test tube experiment, whereby acid is added to the meal and only a small part of the paracetamol remains in the solid content of the meal that precipitates. Apparently, the steep initial increase of paracetamol plasma concentration should not be mistaken for a rapid gastric emptying which would give an overestimation of the rate of gastric emptying.

Part of the paracetamol is encapsulated by the meal and this fraction might be used to determine the gastric emptying. However, after all paracetamol has passed the stomach, still a slow uptake of paracetamol in the plasma is detectable. This indicates that the paracetamol is not instantaneously taken up once the meal containing paracetamol reaches the duodenum. Apparently, paracetamol encapsulated by the meal is only slowly released from the bolus and this slow release appears to be the bottleneck in paracetamol uptake in our experiment. This is also corroborated by the test tube experiment, paracetamol encapsulated by the meal after acid precipitation is only partial released by an extraction. The slow release of the encapsulated paracetamol is probably also the result of the relatively high viscosity of the bolus, which reduces the rate of absorption in the intestine. ${ }^{3,11-}$ 13 In fact, the bioaccessibility, i.e. the release of the compound from the matrix in the intestine, is the rate limiting factor for absorption. This was previously also observed for phenolic compounds in bread. ${ }^{14}$

It is well established that the rate of gastric emptying of high caloric meals is slower than that of low caloric meals. ${ }^{15}$ This is confirmed by the paracetamol concentration time course in the stomach in our study that does demonstrate a slower rate of gastric emptying for the high caloric meals. However, the plasma paracetamol data fail to reflect this difference in gastric emptying of low and high caloric meals. This confirms that for the meals studied, the plasma paracetamol concentration time curve is not a valid marker for gastric emptying. 
Although plasma paracetamol concentrations are an inappropriate measure of the gastric emptying, by definition the paracetamol concentration time curve does reflect the absorption of paracetamol. Actually, our study shows the effect of food on the rate of absorption of paracetamol. The absorption of a drug in the gastrointestinal tract bears major clinical relevance. Often it is advised to take medication in combination with or after a meal to avoid a peak concentration which would prevent side effects and possible toxicity. Our study shows that this is not necessarily the case. In case of enteral feeding still a relatively high peak concentration can arise. Moreover, the duration of the effect might be extended by encapsulation of the drug by the meal and the slow release of the drug from the drug-meal bolus.

Food-drug interactions are not limited to an effect of food on gastric emptying; apparently, it also includes separation of the drug into different fractions, which has an impact on both the onset and duration of the pharmacotherapeutic effect. However, in studying food-drug interactions, it is important to realize that a drug might be separated in two fractions which have a large difference in their rate of absorption. In the use of paracetamol plasma levels as a post prandial marker for gastric emptying, the food-drug interaction on absorption may lead to incorrect conclusions. 


\section{References}

1. Henderson" HRJRRFG. Rang \& Dale's Pharmacology: Churchill Livingstone. 201 1:792.

2. Hunt JN. A possible relation between the regulation of gastric emptying and food intake. Am J Physiol 1980;239:G1-4.

3. Cviiic S, Paroicic J, Langguth P. Viscosity-mediated negative food effect on oral absorption of poorlypermeable drugs with an absorption window in the proximal intestine: In vitro experimental simulation and computational verification. Eur J Pharm Sci. 2014;61:40-53

4. Paroicic J, Vasilievic D, Ibric S, Diuric Z. Tablet disintegration and drug dissolution in viscous media: paracetamol IR tablets. Int J Pharm. 2008;355: 93-99.

5. Welling PG. Effects of food on drug absorption. Annu Rev Nutr. 1996; 16:383-415.

6. Willems $M$, Quartero AO, Numans ME. How useful is paracetamol absorption as a marker of gastric emptying? A systematic literature study. Dig Dis Sci. 200 1;46:2256-2262.

7. Medhus AW, Lofthus CM, Bredesen J, Husebye E. Gastric emptying: the validity of the paracetamol absorption test adjusted for individual pharmacokinetics. Neurogastroenterol Motil. 200 1;13: 79-185.

8. Salden BN, Monserrat V, Troost FJ, et al. Randomised clinical study: Aspergillus niger-derived enzyme digests gluten in the stomach of healthy volunteers. Aliment Pharmacol Ther. 2015;42(3):273-85.

9. Di Pietra AM, Gatti R, Andrisano V, Cavrini V. Application of high-performance liquid chromatography with diode-array detection and on-line post-column photochemical derivatization to the determination of analgesics. J Chromatogr A. 1996;729:355-36 1.

10. Viswanathan CT, Bansal S, Booth B, et al. Quantitative bioanalytical methods validation and implementation: best practices for chromatographic and ligand binding assays. Pharm Res. 2007;24:1962-1973.

11. Heading RC, Nimmo J, Prescott LF, Tothill P. The dependence of paracetamol absorption on the rate of gastric emptying. Br J Pharmacol. 1973;47:415-421.

12. Lentz KA. Current methods for predicting human food effect. AAPS. 2008;10:282-288.

13. Holt $\mathrm{S}$, Heading RC, Carter DC, et al. Effect of gel fibre on gastric emptying and absorption of glucose and paracetamol. Lancet 1979; 1:636-639.

14. Mateo Anson N, Aura AM, Selinheimo E, et al. Bioprocessing of wheat bran in whole wheat bread increases the bioavailability of phenolic acids in men and exerts antiinflammatory effects ex vivo. The Journal of nutrition 2011 1:141:137-143.

15. Gonzalez A, Mugueta C, Parra D, et al. Characterisation with stable isotopes of the presence of a lag phase in the gastric emptying of liquids. Eur J Nutr. 2000;39:224-228. 


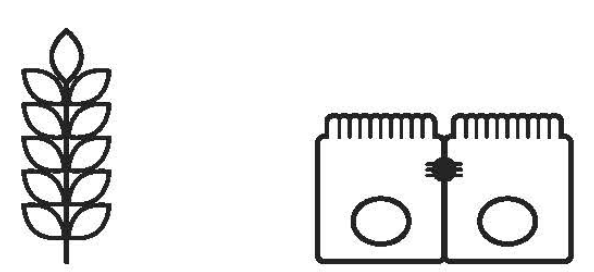

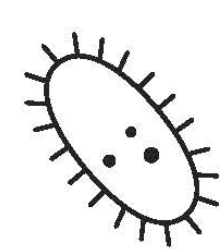




\section{chapter 4}

Reinforcement of intestinal epithelial barrier by arabinoxylans in overweight and obese subjects: a randomized controlled trial

Salden BN, Troost FJ, Wilms E, Truchado P, Vilchez-Vargas R,

Pieper D, Jáuregui R, Marzorati M, van de Wiele T,

Possemiers S, Masclee AA

Clinical Nutrition, article in press 


\section{Abstract}

\section{Background \& aims}

Obesity and metabolic diseases are associated with alterations in microbial composition and impaired gut barrier. Previous in vitro and animal studies have shown that arabinoxylans (AX) have the potential to modulate gut microbiota and gut barrier and therefore could have a protective role. Primary aim of the study was to investigate the effect of AX on intestinal permeability. Secondary aims included the effect of $A X$ on gene transcription and protein expression of tight junctions (TJ), intestinal microbiota composition and activity, immune response and metabolic markers in overweight and obese individuals.

\section{Methods}

In this randomized, double-blind, placebo-controlled trial, 47 overweight subjects were randomly assigned to groups receiving $7.5 \mathrm{~g} / \mathrm{d} A X(n=16), 15 \mathrm{~g} / \mathrm{d} A X(n=17)$ or $15 \mathrm{~g} / \mathrm{d}$ placebo $(n=14)$ for 6 wks. Intestinal permeability was investigated using a multi-sugar test. Sigmoid colon tissue was obtained from a subgroup ( $n=26$ ) for analyzing gene transcription and mucosal expression of TJ proteins. Fecal samples were collected to assess microbial composition and activity. Furthermore, the production of cytokines by stimulated peripheral blood mononuclear cells (PBMCs) was examined. Blood was also sampled for measuring metabolic markers.

\section{Results}

No significant changes in gastrointestinal permeability and TJ protein expression were observed after 6 wks AX supplementation compared to placebo. However, gene transcription of occludin was upregulated in the $7.5 \mathrm{~g} \mathrm{AX}$ group, and transcription of claudin-3 and claudin-4 were upregulated in the $15 \mathrm{~g}$ AX group compared to placebo. Furthermore, fecal microbiota diversity was decreased after 6 wks $15 \mathrm{~g}$ AX treatment, but no change in relative abundance of dominant phyla was observed. AX intake significantly decreased fecal $\mathrm{pH}$ and increased fecal concentrations of total SCFAs, acetate, propionate and butyrate, compared to placebo. Additionally, a decreased TNFa production by stimulated PBMCs was observed after $15 \mathrm{~g} \mathrm{AX}$ treatment. No changes in metabolic markers were detected.

\section{Conclusions}

Regular consumption of AX resulted in a more beneficial fermentation profile in overweight and obese individuals. Further studies are required to assess whether such fermentation profile will translate into improved gut barrier function and immune health. 


\section{Introduction}

The gastrointestinal (Gl) epithelial barrier is a complex system that includes an active local immune defense, a physical barrier comprised of a network of epithelial cells which are connected by tight junction (TJ) proteins to control permeability, a mucus layer, secretion of antimicrobial peptides, intestinal microbiota and luminal microbial metabolism.' Recent research has focused on intestinal microbiota and their effects on intestinal physiology, nutrient digestion, luminal metabolism and immune function. ${ }^{2}$ Short-chain fatty acids (SCFA) are the end products of fermentation of non-digestible carbohydrates by intestinal microbiota and have an important role in maintenance of intestinal homeostasis. Changes in gut microbiota composition may affect gut barrier function via changes in expression, localization and distribution of TJ proteins, thereby influencing gut permeability. ${ }^{3}$ Changes in gut barrier function are regarded as early events or triggers in the development of various intestinal diseases, and also in the development of systemic metabolic diseases, such as diabetes mellitus type 2 (DM2) and cardiovascular diseases (CVD). ${ }^{4}$

Modulation of gut microbial communities by prebiotics appears to be an interesting concept to enhance the gut barrier, to treat or even prevent the onset or aggravation of chronic diseases. Prebiotics are non-digestible food ingredients selectively stimulating growth and/or activity in the GI microflora conferring potential health benefits to the host. ${ }^{5} \mathrm{~A}$ limitation of most prebiotics is their rapid fermentation in the proximal colon. Arabinoxylans (AX), the most abundant non-digestible carbohydrates present in wheat, form a novel class of potential prebiotics. $^{6}$ Due to their structure, AX rely on a whole spectrum of (microbial) enzymes for degradation. They are more gradually fermented along the colon, resulting in more distal fermentation. ${ }^{6.7} A X$ exist in different forms, ranging from long-chains to enzymatically modified short-chain fractions. In vitro research has shown that the AX form affects the fermentation pattern and immune function, high-molecular weight $A X$ being most active. ${ }^{7.8}$ Promising outcomes of high-molecular weight $A X$ on the gut barrier, 9.10 gut microbiota, $9.11,12$ immune system ${ }^{9}$ and metabolic markers ${ }^{9}$ have been shown both in in vitro and in animal studies. To date, no human data are available on the combined effect of such $A X$ on intestinal environment, gut barrier and immune system.

Therefore, the purpose of this study was to investigate the effects of a high-molecular weight $A X$ concentrate on the gut barrier, intestinal microbiota, immune system and metabolic control in humans in overweight and obese individuals. We hypothesized that 6 wks AX supplementation ameliorates the gut barrier, improves colonic microbiota composition and its metabolic activities, improves the immune system, enhances metabolic control and is well-tolerated in overweight and obese individuals. We specifically chose to study an overweight and obese population, as overweight and obesity are associated with an impaired gut barrier function. Primary aim of this study was to investigate the effect of AX on intestinal permeability, measured by a multi-sugar test. Secondary, we aimed to 
investigate the effect of $A X$ intake on gene transcription and protein expression of $T J$, on fecal microbiota composition and fecal pH, ammonium and SCFA concentrations, on cytokine production by stimulated peripheral blood mononuclear cells (PBMCs), on blood concentrations of glucose, insulin and lipids, and on Gl tolerance.

\section{Materials and methods}

The Medical Ethics Committee of the Maastricht University Medical Center (MUMC+) approved the trial and the study was performed at the MUMC+ from March 2012 to December 2012 in full accordance with the principles of the Declaration of Helsinki of 1964 as amended in 2013 and with the Dutch Regulations on Medical Research involving Human Subjects (WMO, 1998). Before participation all participants provided written informed consent.

\section{Subjects}

Healthy overweight and obese volunteers aged 18-70 y with a BMI between 28-35 kg/m² were recruited by local advertisement. Key exclusion criteria were: any medical condition that might interfere with the study and might jeopardise the health status of the participant; smoking; abuse of alcohol (>20 consumptions/wk) and drugs; no consistently stable body weight for at least 6 months $( \pm 2 \mathrm{~kg}$ ); plans to lose weight or following an energy restriction diet during study period; use of medication, vitamin- or mineral supplements, consumption of pro-, pre- or synbiotics during study period; use of antibiotics in the 90 days prior to start of the study; pregnancy and lactation; history of any side effects towards the intake of pro-, pre-, or synbiotic supplements of any kind. Throughout the trial, subjects consumed their habitual diet. Before start of the study, subjects were informed about the prohibited pre-, pro- and synbiotics and the food products containing them.

To assess the difference in $G$ permeability between this study population and a group of age and sex matched healthy lean subjects, we used the Gl permeability data obtained with exactly the same protocol in healthy controls. These data have recently been published by our group. ${ }^{13}$

\section{Design and intervention}

This study was set up as a randomized, placebo-controlled, double-blind, parallel group study. Each subject was tested on three occasions, while a subset of these subjects additionally provided sigmoid mucosal tissue samples on two separate occasions. Participants were randomly assigned in a double-blind fashion to one of the three 
intervention arms: $7.5 \mathrm{~g}$ AX, $15 \mathrm{~g}$ AX or a placebo (15 g maltodextrin). An independent and blinded person generated the randomization list, using a computerized method. All participants and investigators remained blind to intervention until all analyses were finalized. Participants were instructed to refrain from consumption of alcohol and strenuous physical exercise on the day before each test day. After an overnight fast, subjects handed in a fecal sample on the first test day. Fecal samples were collected one day before or on a test day, and stored at $-20^{\circ} \mathrm{C}$ until arrival at the study site. Subsequently, venous blood samples were collected from an antecubital vein in the fore-arm. Then, subjects ingested a multisugar drink, to assess gastrointestinal permeability, and collected full urine output for $24 \mathrm{~h}$. A subset of the participants underwent a standard flexible sigmoidoscopy without bowel preparation on the second test day. Seven mucosal tissue samples of approximately $5 \mathrm{mg}$ each were obtained from the sigmoid colon by using a standard forceps (diameter: $2.8 \mathrm{~mm}$ ) and directly after collection frozen in liquid nitrogen. Two tissue samples were embedded in Tissue-tek (Sakura Finetek, Tokio, Japan) prior to freezing for immunohistochemical analyses. After completion of the baseline measurements, participants received the study product for the first 3 wks. After 3 wks of daily supplementation, the third test day was organized. Again, a fecal sample was handed in, blood samples were taken and study products for another 3 wks were provided. After 6 wks of daily administration of the study product, the fourth and fifth test days took place. Measurements were identical to the baseline measurements performed during the first and second test days, respectively. The same subgroup of subjects underwent again a sigmoidoscopy. Also, all participants had to complete a questionnaire at weekly intervals, to assess the presence of $\mathrm{Gl}$ symptoms, stool frequency and stool consistency. To assess compliance, participants were asked to collect the empty sachets and to return these at the last visit.

\section{AX concentrate and placebo}

BioActor BV (Maastricht, The Netherlands) supplied AX with a purity of $62 \%$, degree of substitution of 0.7 , and a varying degree of polymerization, with an average above 60; the composition of the batch used for the study was $67 \%$ non-starch polysaccharides (62\% AX), 18\% protein, 0.5\% lipids, 3.8\% ash. Maltodextrin (Glucidex IT 19; Roqette Frères, Lestrem Cedex, France) served as placebo. The study products were provided to subjects as a powder in sachets. The placebo group received per day two sachets, each containing $7.5 \mathrm{~g}$ Maltodextrin, the $7.5 \mathrm{~g}$ AX group received per day two sachets, each containing $3.75 \mathrm{~g} \mathrm{AX}$ combined with $3.75 \mathrm{~g}$ Maltodextrin and the $15 \mathrm{~g}$ AX group received per day two sachets, each containing $7.5 \mathrm{~g}$ AX. Subjects were asked to stir the content of one sachet in $200 \mathrm{ml}$ liquid (e.g. juice, water, milk) and ingest the solution twice daily (every morning and every evening) for 6 wks. 


\section{Gastrointestinal permeability}

Gl permeability was investigated using a validated multi-sugar test, quantifying 24-h urinary excretion of five different ingested sugars reflecting the permeability of 4 segments of the Gl tract. Firstly, fasted volunteers emptied their bladder and drank the multi-sugar drink, consisting of $1 \mathrm{~g}$ lactulose (Centrafarm, Etten-Leur, the Netherlands), $0.5 \mathrm{~g}$ L-rhamnose (Danisco, Copenhangen, Denmark), 1 g erythritol (Now Foods, Bloomindale, IL, USA), $1 \mathrm{~g}$ sucralose (Brenntag, Sittard, the Netherlands) and 1 g sucrose (Van Gilse, Dinteloord, the Netherlands) dissolved in $150 \mathrm{~mL}$ tap water. Participants were instructed to collect their 24 $\mathrm{h}$ urine output in two different fractions; one fraction containing the $0-5 \mathrm{~h}$ urine output and a second fraction containing the 5-24 h urine output. It was not allowed to ingest any foods or drinks, except for water, throughout the first urine fraction (0-5 h) collection. Gastroduodenal and small intestinal permeability were reflected by the $0-5 \mathrm{~h}$ urinary sucrose excretion and the lactulose and L-rhamnose (L/R) ratio, respectively. In the 5-24 $\mathrm{h}$ urine, the sucralose to erythritol (S/E) ratio represented colonic permeability, while in the O$24 \mathrm{~h}$ urine it indicated whole gut permeability. After collection of the $24 \mathrm{~h}$ urine output, total volume was quantified and urine aliquots were frozen at $-80^{\circ} \mathrm{C}$. Urinary sugars were analysed using HPLC-MS as reported earlier. ${ }^{14}$ The intestinal permeability test and the analysis of urine samples in the study of Mujagic et al. ${ }^{13}$ was performed in exactly the same way and done by the same research group as in our study.

\section{Gene transcription of TJ and associated proteins}

Gene transcription of TJ and associated proteins (claudin-3, claudin-4, occludin, myosin light-chain kinase (MLCK) and zonula occludens- 1 (ZO-1)) in sigmoid colon tissue samples was determined by quantitative real-time polymerase chain reaction, as earlier presented by Pijls et al. ${ }^{15}$ Housekeeping genes included GAPDH and 18S RNA. Data are shown as normalized expression ratios.

\section{Protein concentrations of claudin-3}

Sigmoid mucosal tissue was used for the determination of claudin-3 protein concentrations. Biopsy specimens were kept in liquid nitrogen and ground in a nitrogen cold mortar. The powder was gently scraped with a spatula of the auger and dissolved in $150 \mu$ phosphatebuffered saline (PBS) (Invitrogen 10010, pH 7.4) containing protease inhibitor cocktail (Sigma P8340) with a concentration from $10 \mu \mathrm{l} \mathrm{Pl/ml} \mathrm{PBS} \mathrm{and} \mathrm{centrifuged} \mathrm{for} 20$ min at $10,000 \mathrm{rpm}$ at $4^{\circ} \mathrm{C}$. Supernatant was stored at $-80^{\circ} \mathrm{C}$. The concentration of claudin-3 was determined with the sandwich ELISA kit for human claudin-3 ICloud Clone Corp., Houston USA Kit: SEF293Hu). The microtitre plate provided in this kit was precoated with an 
antibody specific to claudin-3. Standards of samples (samples were 1 to 5 diluted) were added to the suitable microtitre plate wells together with a claudin-3 specific biotin-conjugated antibody. Then, avidin conjugated to horseradish peroxidase was applied on each microplate well and incubated. Subsequently tetramethylbenzidine substrate solution was added, and only the wells containing claudin-3, biotin-conjugated antibody and enzyme-conjugated avidin exhibited a color change. Addition of sulphuric acid solution ended the enzyme-substrate reaction. The change in color was quantified using spectrophotometry (wavelength: $450 \mathrm{~nm} \pm 10 \mathrm{~nm}$ ). The concentrations of claudin-3 in the tissue was defined by comparison of the O.D. of the tissue sample to that of the standard curve. Total protein determination in the sigmoid tissue was done using Pierce ${ }^{\circledast}$ BCA Protein Assay Kit (ThermoFisher, Waltham, MA USA). Samples were 1 to 15 diluted. Data are presented as pg claudin-3 per ug total protein.

\section{Immunofluorescence staining of ZO- 1 and occludin}

Immunofluorescent staining of ZO- 1 and occludin was done as presented earlier. ${ }^{16}$ Leica TCS SPE confocal microscopy (Leica Micro systems GmbH, Mannheim, Germany) was used to analyse images. Obtained images were quantified by calculating the AUC. Briefly, to assess the fluorescent staining of both TJ proteins in the TJ region, mucosal tissue was exposed to confocal analysis of $Z$ regions perpendicular to the apical cell surface of the epithelium. Three randomly selected areas within 3 different $Z$ regions were analyzed. The middle part of the villi was used for analyzing the staining. Image J software created the plot profiles reflecting staining intensity and AUC was calculated using Matlab R2013a software (Natick, MA, USA).

\section{Microbiota composition and activity}

\section{DNA extraction}

To extract DNA from fecal samples $(100 \mathrm{mg}$ ) samples were resuspended in $1 \mathrm{~mL}$ of lysis buffer (Tris/HCl (100 mM pH 8.0), 100 mM EDTA, $100 \mathrm{mM} \mathrm{NaCl,} \mathrm{1 \%} \mathrm{(wt/vol)}$ polyvinylpyrrolidone and 2\% (wt/vol) SDS). Cells were lysed in a Fast Prep ${ }^{\circledR}$-24 instrument (MP Biomedicals, Solon, OH, USA) for $30 \mathrm{~s}$ at $1800 \mathrm{rpm}$ and extractions were performed using phenol-chloroform-isoamyl alcohol (25:24:1). DNA was precipitated with ice-cold isopropanol and resuspended in $100 \mu$ of TE buffer $110 \mathrm{mM}$ Tris-HCl, $1 \mathrm{mM}$ EDTA [pH 8.0]). The amount and quality of DNA was measured using an ND 1000 spectrophotometer (ThermoFisher, Waltham, MA, USA). 


\section{Gut microbiota composition}

We used 165 targeted DNA-based Illumina with MiSeq platform to assess the gut microbiome structure in the fecal samples. Primers for the 16S rRNA gene amplified the hypervariable region V 1-V2. After sequencing, quality filtering was performed as previously described and sequences were taxonomically annotated using RDP database with 80\% threshold. ${ }^{17}$ Sequencing depth was normalized to the minimum reads per sample, using the Phyloseq package from R. Rarefaction curves and biodiversity indices were calculated using the vegan package from R. Rarefaction curve analysis showed that the use of at least 10.000 reads was sufficient to sample the complete diversity of the library.

The following metrics of alpha diversity were determined: observed richness, Shannon diversity index and Simpson evenness. Beta diversity was assessed by Bray-Curtis (BC) dissimilarity. $\mathrm{BC}$ is an ecological diversity index representing the extent of change in microbiota composition. This index takes into consideration both the bacterial taxa detected in a specimen, and their relative presence.

\section{Fecal pH, ammonium and SCFA determination}

Fecal samples were collected by the participants and stored at $-20^{\circ} \mathrm{C}$ until handed in at the study site. For SCFA and ammonium analyses, aliquots of approximately $1 \mathrm{~g}$ of fecal samples were diluted and homogenized with $6 \mathrm{~mL}$ demineralized water. After removal of the particulate material by centrifugation ( $10 \mathrm{~min}, 500 \times \mathrm{g}$ ), the supernatants were stored at $-20^{\circ} \mathrm{C}$ prior to analysis. SCFA concentrations in the supernatants were determined using gas chromatography with flame ionization detector, based on the SCFA concentration analysis described by Possemiers et al.. ${ }^{18}$ The sum of acetate, propionate, butyrate, isobutyrate, valerate, isovalerate, caproate and isocaproate represent total SCFA. Ammonia concentrations in the supernatants were measured using the method previously presented. ${ }^{19}$ To determine fecal $\mathrm{pH}$, an aliquot of approximately $1 \mathrm{~g}$ feces was homogenized by mixing into $10 \%(\mathrm{w} / \mathrm{w})$ demineralized water. Directly after homogenization $\mathrm{pH}$ was assessed.

\section{Production of cytokines by stimulated peripheral blood mononuclear cells}

Blood was collected in sodium heparine tubes (Becton \& Dickinson, Franklin Lakes, NJ, USA) and used within $1 \mathrm{~h}$ for the isolation of PBMCs. To examine ex vivo cytokine production by PBMCs, cells were isolated from whole blood and stimulated with Phytohemagglutinin-M (PHA). PHA-stimulation is used to bind antigen-presenting cells and T-cells and thereby PHA induces T-cell proliferation. This technique is used to enlarge the current activities of the T-cells present in the culture. Lymphoprep gradient centrifugation was performed according to the manufacturer's instructions (Takeda Nederland B.V., Hoofddorp, The Netherlands). Freshly isolated cells were diluted in RPMI 1640 culture medium containing HEPES and 
L-glutamine (ThermoFisher, Waltham, MA USA), with 1\% added Penicillin/Streptomycin, Sodium Pyruvate, and heat inactivated human serum pool. Cells were seeded $1^{*} 10^{6}$ in 24-well flat bottom culture plates (Greiner Bio-One B.V., Alphen a/d Rijn, The Netherlands) and T-cell proliferation was stimulated with PHA (Roche Diagnostics Nederland B.V., Almere, The Netherlands). After $48 \mathrm{~h}$, the culture medium was harvested and stored at $-80^{\circ} \mathrm{C}$ until further analysis. Interferon-gamma (IFN $\gamma$ ) was measured using a sandwich ELISA according to the manufacturer's instructions (eBioscience, Vienna, Austria). IL-2, IL-10, IL-12p40 and TNFa were measured with a multiplex chemoluminescence assay Meso Scale Diagnostics, Rockville, Maryland USA).

\section{Blood lipids, glucose and insulin}

Blood samples were collected at baseline, after 3 wks of supplementation and at the end of the supplementation period. Serum concentrations of glucose, total cholesterol, LDL- and $\mathrm{HDL}$-cholesterol and triglycerides were measured using spectrophotometry. Plasma insulin was determined using a luminescence-enhanced immunoenzymatic assay. Insulin sensitivity was estimated by the quantitative insulin sensitivity check index (QUICKI) index as: 1 / (log insulin $\mathrm{O} \mathrm{h}+\log$ glucose $\mathrm{O} \mathrm{h}$ ).

\section{Gastrointestinal tolerance}

The gastrointestinal symptom rating scale (GSRS) was used to assess the presence of GI symptoms. This questionnaire consists of 15 seven-point graded items that can be combined into 5 symptom clusters reflecting reflux, abdominal pain, indigestion, diarrhea, and constipation. The absence of troublesome symptoms is represented by a score of 1 , while a score of 7 reflects very inconvenient $G$ symptoms. ${ }^{20}$ Defecation frequency and stool consistency were assessed using the Bristol stool chart. Subjects completed these questionnaires at weekly intervals during the study period.

\section{Statistical analyses}

The primary outcome of the study was the effect of 6 wks $7.5 \mathrm{~g} \mathrm{AX}$ and $15 \mathrm{~g} \mathrm{AX}$ supplementation on GI permeability, compared to placebo. Secondary outcomes include the effect of 6 wks $A X$ supplementation versus placebo on gene transcription and protein expression of TJ, fecal microbiota composition and activity, cytokine production by PBMCs, metabolic markers and on Gl tolerance. Sample size was determined for the primary outcome of the study, using a significance level alpha $=0.017$ and a power of $80 \%$. A study in diet-induced obese mice, investigating the effect of a dietary fiber on $\mathrm{Gl}$ permeability, ${ }^{21}$ was used for calculating the sample size. To detect a difference in intestinal permeability of 
$0.3 \pm 0.25 \mu \mathrm{g} / \mathrm{ml}$ (mean $\pm \mathrm{SD}$ ) a total of 45 subjects would be required. Baseline characteristics are displayed as mean (SD) for numerical variables. Baseline values in age and BMI between overweight and obese individuals and lean controls were compared using independent t-test, and baseline numbers in sex were evaluated with chi-square test. Differences in gut barrier (function), fecal pH, relative abundance of main phyla of the fecal microbiota, alpha diversity metrics, fecal SCFA concentrations, immune response, blood parameters and digestive tolerance between placebo and $7.5 \mathrm{~g} \mathrm{AX}$ and placebo and $15 \mathrm{~g}$ AX were assessed using linear mixed models with group (placebo, $7.5 \mathrm{~g} A X$ and $15 \mathrm{~g} \mathrm{AX}$ ), time 10,3 and 6 wks for $A X$ effect) and group*time as fixed factors, where an unstructured covariance structure was used for repeated measures. The linear mixed model accounts for the correlation between repeated measures and missing data, where a likelihood approach was used assuming data missing at random.

Gl permeability data were not normally distributed, as assessed by Shapiro-Wilk Test. Mann-Whitney U test was used to evaluate the difference in baseline $G$ l permeability between healthy lean and overweight and obese individuals. Statistical analysis was performed using IBM SPSS Statistics for Windows (version 21.0, Armonk, NY, USA).

$\mathrm{R}$ language for statistical computing [http://www.R-project.org] was used for normalizing sequencing depth, calculating rarefaction curves and biodiversity indices. It was also used to generate exploratory heat maps, using the heatmap. 2 function from the $R$ package gplots on logarithmic transformed data. To determine the effect of intervention on the shared diversity between samples, beta diversity metrics (BC dissimilarity) were calculated for placebo versus $7.5 \mathrm{~g} \mathrm{AX}$ and placebo versus $15 \mathrm{~g}$ AX. A Principal coordinate analysis (PCoA), based on the BC similarity distances, was used in order to visualize the microbial variation among placebo, $7.5 \mathrm{~g} \mathrm{AX}$ and $15 \mathrm{~g} \mathrm{AX}$. A two-way crossed analysis of similarity (ANOSIM) was used to determine if the microbial communities were significantly different between treatment groups. These analyses were performed using PAleontological STatistics version 3.11 (PAST, Øyvind Hammer, Natural History Museum, University of Oslo). For all comparisons, two-sided p-values $\leq 0.05$ were considered statistically significant.

\section{Results}

\section{Study subjects}

A total of 47 healthy volunteers were enrolled in the study; 14 subjects were assigned to the placebo group, 16 subjects to the AX $7.5 \mathrm{~g}$ group and 17 subjects to the AX $15 \mathrm{~g}$ group. Forty-five volunteers completed the entire study protocol. One participant dropped out during the intervention period because of a pneumonia and consequent antibiotic treatment. The second dropout developed gallstones during the study period and had to 
undergo surgery. From these participants only baseline characteristics were available Online Supplemental Material Figure S4.1). Sigmoidoscopy was performed in 9 participants in the placebo group, 9 participants in the $7.5 \mathrm{~g} \mathrm{AX}$ group and 8 participants in the $15 \mathrm{~g}$ AX group. Baseline characteristics are presented in Table 4.1.

Data of a group of 33 lean age and sex matched controls, derived from Mujagic et al., ${ }^{13}$ were used to evaluate differences in $G$ permeability between lean and overweight/obese subjects.

Table 4.1 Baseline characteristics in the overweight and obese study population, and lean controls.

\begin{tabular}{lccccc}
\hline & $\begin{array}{c}\text { Overall cohort } \\
(n=47)\end{array}$ & $\begin{array}{c}\text { Placebo } \\
(n=14)\end{array}$ & $\begin{array}{c}\text { AX 7.5 g } \\
(n=16)\end{array}$ & $\begin{array}{c}\text { AX 15 g } \\
(n=17)\end{array}$ & $\begin{array}{c}\text { Lean controls } \\
(n=33)\end{array}$ \\
\hline Age $(\mathrm{y})$ & $48 \pm 16$ & $49 \pm 17$ & $49 \pm 17$ & $47 \pm 15$ & $42 \pm 3$ \\
Sex $(\mathrm{M} / \mathrm{F})$ & $25 / 22$ & $8 / 6$ & $10 / 6$ & $7 / 10$ & $13 / 20$ \\
BMI $\left(\mathrm{kg} / \mathrm{m}^{2}\right)$ & $31.0 \pm 2.4$ & $31.4 \pm 3.1$ & $30.2 \pm 1.9$ & $31.5 \pm 2.2$ & $22.0 \pm 0.31$ \\
Sigmoidoscopy $(n)$ & 26 & 9 & 9 & 8 & $\mathrm{n} / \mathrm{a}$ \\
\hline
\end{tabular}

Differences in age and BMI between overall cohort and lean controls tested with independent t-test, differences in sex between overall cohort and lean controls tested with chi-square test. Data are given as mean $\pm \mathrm{SD}$. $P<0.001$ overall cohort vs. lean controls. AX, arabinoxylans.

\section{Gastrointestinal permeability}

Baseline Gl permeability was significantly increased in our healthy, but overweight population, compared to an age and sex matched healthy, lean population lall $P<0.001$, Table 4.2).

Table 4.2 Gastrointestinal permeability, reflected by urinary sugar excretion ( $\mu$ mol) and ratios of excreted sugars (in 0-5, 5-24 and 0-24 h fraction) for lean, and overweight and obese subjects.

\begin{tabular}{lccc}
\hline Sugar excretion & Lean $(n=33)$ & Overweight \& obese $(n=45)$ & $P$ value \\
\hline O-5 h sucrose & $2.06[0.51 ; 6.91]$ & $7.75[4.68 ; 13.91]$ & $<0.001$ \\
O-5 h L/R ratio & $0.016[0.009 ; 0.036]$ & $0.050[0.030 ; 0.108]$ & $<0.001$ \\
5-24 h S/E ratio & $0.009[0.006 ; 0.015]$ & $0.018[0.014 ; 0.023]$ & $<0.001$ \\
0-24 h S/E ratio & $0.010[0.005 ; 0.014]$ & $0.020[0.014 ; 0.024]$ & $<0.001$ \\
\hline
\end{tabular}

Differences between lean subjects and overweight and obese subjects tested with Mann-Whitney U test. Data are given as median [IQR, i.e. Q 1; Q3]. L/R, lactulose/l-rhamnose. S/E, sucralose/erythritol.

\section{Six weeks effect}

Table 4.3 presents the effect of 6 wks intervention on $G$ permeability. Gastroduodenal permeability, as indicated by the sucrose excretion in the 0-5 $\mathrm{h}$ urine fraction, did not significantly change after both $7.5 \mathrm{~g} A X$ and $15 \mathrm{~g} A X$ supplementation compared to placebo (all $P \geq 0.224$ ). Neither was the $0-5 \mathrm{~h}$ urinary L/R ratio, reflecting small intestine permeability, altered after the $A X$ interventions compared to placebo (all $P \geq 0.219$ ). 
Colonic and whole gut permeability, respectively reflected by the S/E ratio in 5-24 h and the $0-24$ h urine fractions, did not show significant differences among $A X$ and placebo groups after 6 wks supplementation (all $P \geq 0.257$ ).

Table 4.3 Gastrointestinal permeability, reflected by urinary sugar excretion ( $\mu$ mol) and ratios of excreted sugars (in 0-5, 5-24 and 0-24 h fraction) at baseline and after 6 wks supplementation.

\begin{tabular}{lcccc}
\hline Sugar excretion & Intervention & Baseline & End & $P$ value \\
\hline 0-5 h sucrose & Placebo & $5.43[3.97 ; 8.74]$ & $7.10[4.99 ; 11.75]$ & \\
& $7.5 \mathrm{~g} \mathrm{AX}$ & $10.76[8.39 ; 21.89]$ & $12.52[8.38 ; 17.55]$ & 0.256 \\
& $15 \mathrm{~g} \mathrm{AX}$ & $7.05[3.38 ; 13.24]$ & $7.88[4.99 ; 9.29]$ & 0.224 \\
0-5 h L/R ratio & Placebo & $0.045[0.030 ; 0.073]$ & $0.065[0.038 ; 0.170]$ & \\
& $7.5 \mathrm{~g} \mathrm{AX}$ & $0.070[0.040 ; 0.120]$ & $0.060[0.048 ; 0.135]$ & 0.464 \\
& $15 \mathrm{~g} \mathrm{AX}$ & $0.050[0.030 ; 0.115]$ & $0.065[0.040 ; 0.095]$ & 0.219 \\
$5-24 \mathrm{~h} \mathrm{S/E} \mathrm{ratio}$ & Placebo & $0.020[0.014 ; 0.025]$ & $0.018[0.016 ; 0.023]$ & \\
& $7.5 \mathrm{~g} \mathrm{AX}$ & $0.016[0.012 ; 0.020]$ & $0.020[0.013 ; 0.024]$ & 0.766 \\
& $15 \mathrm{~g} \mathrm{AX}$ & $0.020[0.014 ; 0.026]$ & $0.017[0.013 ; 0.024]$ & 0.810 \\
0-24 h S/E ratio & Placebo & $0.020[0.015 ; 0.025]$ & $0.017[0.015 ; 0.020]$ & \\
& $7.5 \mathrm{~g} \mathrm{AX}$ & $0.018[0.014 ; 0.022]$ & $0.018[0.013 ; 0.025]$ & 0.257 \\
& $15 \mathrm{~g} \mathrm{AX}$ & $0.020[0.014 ; 0.024]$ & $0.016[0.014 ; 0.024]$ & 0.986 \\
\hline
\end{tabular}

Differences between placebo, $7.5 \mathrm{~g} \mathrm{AX}$ and $15 \mathrm{~g} A X$ tested with linear mixed model with correction for baseline values. Data are given as median $[I Q R$, i.e. Q1; $Q 3]$. AX, arabinoxylans. L/R, lactulose/l-rhamnose. S/E, sucralose/erythritol.

\section{Gene transcription of TJ and associated proteins}

Results regarding the effects of $A X$ administration on gene transcription of TJ and associated proteins are given in Table 4.4. Transcription of claudin-3 and claudin-4 was significantly upregulated in participants receiving daily $15 \mathrm{~g}$ AX for $6 \mathrm{wks}$, compared to participants receiving placebo $(P=0.012$ and $P=0.046$ respectively). Furthermore, a trend towards upregulation of the TJ protein occludin was seen after $15 \mathrm{~g} \mathrm{AX}$ intake compared to placebo ( $P=0.057)$. In the $7.5 \mathrm{~g} A X$ group we observed a significant upregulation of occludin compared to placebo $(P=0.031)$. No significant changes in gene transcription for MLCK and ZO- 1 between both AX groups and the placebo group were observed lall $P \geq$ 0.087).

\section{Protein concentration of claudin-3, ZO- 1 and occludin}

At baseline, protein concentrations of claudin-3 were $6.10 \pm 0.61$ (mean \pm SEM), $4.92 \pm$ 0.41 and $7.02 \pm 1.49 \mathrm{pg}$ claudin-3 per ug protein for placebo, $7.5 \mathrm{~g} \mathrm{AX}$ and $15 \mathrm{~g} \mathrm{AX}$ respectively. Protein concentration of $\mathrm{ZO}-1$ and occludin in biopsies were quantified by calculating an AUC. At baseline, AUCs of ZO- 1 were $159 \pm 34$ (mean \pm SEM), $126 \pm 33$ and $177 \pm 41$ and of occludin $112 \pm 19,142 \pm 35$ and $140 \pm 29$ for placebo, $7.5 \mathrm{~g} \mathrm{AX}$ and $15 \mathrm{~g} A X$ respectively. No significant differences were observed in sigmoid protein 
concentrations of claudin-3, ZO- 1 and occludin between placebo and $A X$ interventions after 6 wks supplementation (all $P \geq 0.144$, data not shown).

Table 4.4 Gene transcription of TJ and associated proteins (expressed as normalised expression ratios) at baseline and after 6 wks supplementation.

\begin{tabular}{lcccccccc}
\hline $\begin{array}{l}\text { Gene } \\
\text { transcription }\end{array}$ & Intervention & \multicolumn{2}{c}{ Baseline } & \multicolumn{2}{c}{ End } & Difference & $95 \% \mathrm{Cl}$ & $P$ value \\
\hline Claudin-3 & Placebo & 1.13 & 0.06 & 1.12 & 0.04 & & & \\
& $7.5 \mathrm{~g} \mathrm{AX}$ & 1.09 & 0.04 & 1.13 & 0.03 & 0.02 & $-0.01,0.05$ & 0.225 \\
& $15 \mathrm{~g} \mathrm{AX}$ & 1.12 & 0.05 & 1.16 & 0.04 & 0.05 & $0.01,0.08$ & 0.012 \\
Claudin-4 & Placebo & 1.13 & 0.04 & 1.11 & 0.03 & & & \\
& $7.5 \mathrm{~g} \mathrm{AX}$ & 1.10 & 0.04 & 1.12 & 0.03 & 0.02 & $-0.01,0.05$ & 0.152 \\
& $15 \mathrm{~g} \mathrm{AX}$ & 1.10 & 0.05 & 1.13 & 0.04 & 0.03 & $0.00,0.06$ & 0.046 \\
MLCK & Placebo & 1.08 & 0.06 & 1.08 & 0.03 & & & \\
& $7.5 \mathrm{~g} \mathrm{AX}$ & 1.06 & 0.03 & 1.10 & 0.03 & 0.03 & $0.00,0.06$ & 0.087 \\
& $15 \mathrm{~g} \mathrm{AX}$ & 1.06 & 0.06 & 1.09 & 0.05 & 0.02 & $-0.01,0.05$ & 0.236 \\
& Placebo & 1.14 & 0.05 & 1.14 & 0.02 & & & \\
& $7.5 \mathrm{~g} \mathrm{AX}$ & 1.13 & 0.03 & 1.17 & 0.03 & 0.04 & $0.00,0.07$ & 0.031 \\
& $15 \mathrm{~g} \mathrm{AX}$ & 1.15 & 0.07 & 1.17 & 0.05 & 0.03 & $-0.01,0.07$ & 0.057 \\
& Zlacebo & 1.17 & 0.05 & 1.17 & 0.04 & & & \\
& $7.5 \mathrm{~g} \mathrm{AX}$ & 1.15 & 0.04 & 1.19 & 0.04 & 0.02 & $-0.01,0.05$ & 0.197 \\
& $15 \mathrm{~g} \mathrm{AX}$ & 1.14 & 0.05 & 1.17 & 0.04 & 0.01 & $-0.02,0.05$ & 0.516 \\
\hline
\end{tabular}

Differences between placebo, $7.5 \mathrm{~g}$ AX and $15 \mathrm{~g} \mathrm{AX}$ tested with linear mixed model. The presented difference in means is corrected for baseline differences. AX, arabinoxylans. MLCK, myosin light-chain kinase. OCLN, occludin. ZO- 1, zonula occludens.

\section{Gut microbiota composition}

From 37 study participants (placebo: $n=12 ; 7.5 \mathrm{~g} A X: n=9 ; 15 \mathrm{~g} A X: n=15)$ gut microbiota composition data were available. Data of 8 participants were not available because there was no amplification in sequencing or data were removed due to quality control issues.

\section{Phylum relative abundance}

165 targeted DNA-based Illumina with MiSeq platform allowed to obtain at least 30.000 usable reads per sample. The dominant phyla across all baseline fecal samples were firmicutes (mean 52.5\%, SD 10.5\%) and bacteroidetes (mean 36.0\%, SD 10.1\%), followed by proteobacteria (mean $7.7 \%$, SD 7.6\%) and actinobacteria (mean 3.0\%, SD 2.9\%). The relative abundance of these four dominant phyla did not change significantly after 6 weeks intake of $7.5 \mathrm{~g} \mathrm{AX}$ or $15 \mathrm{~g} \mathrm{AX}$, compared to placebo (all $P \geq 0.061$; Figure 4.1). 


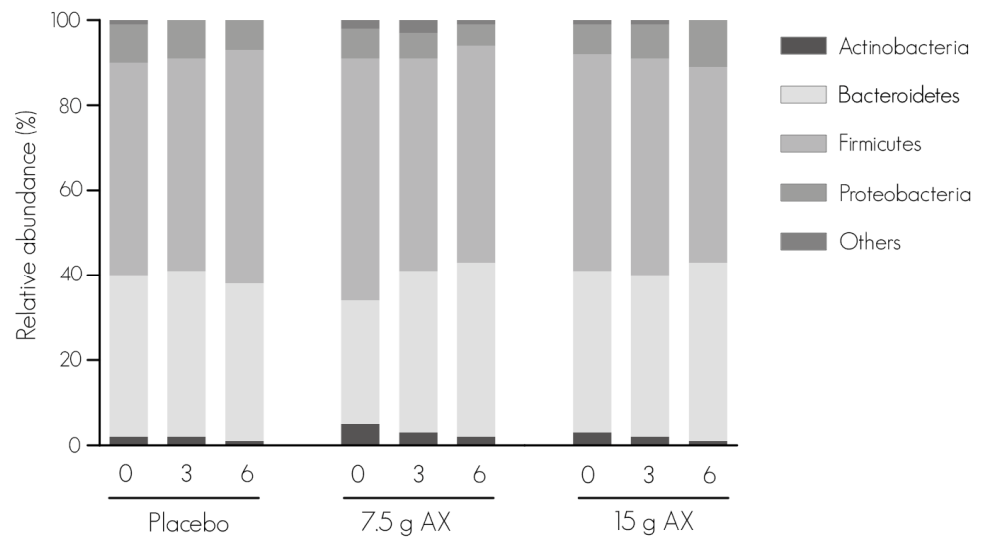

Figure 4.1 Relative abundance of the main phyla according the 16S-targeted Illumina data for placebo, $7.5 \mathrm{~g}$ $\mathrm{AX}$ and $15 \mathrm{~g} \mathrm{AX}$ at baseline (0), after 3 wks supplementation (3) and after 6 wks supplementation (6). No significant differences in relative abundance between placebo, $7.5 \mathrm{~g} A X$ and $15 \mathrm{~g} A X$ were during study period observed, as tested with linear mixed model with correction for baseline values. AX, arabinoxylans.

\section{Fecal samples alpha diversity}

Six wks treatment with the two dosages $A X$ did not change the Simpson evenness index, compared to placebo (all $P \geq 0.063$, Table 4.5). However, 6 wks intake of $15 \mathrm{~g}$ AX but not $7.5 \mathrm{~g}$ AX significantly reduced the observed richness and lowered Shannon diversity, compared to placebo $(P=0.029$ and $P=0.036$ respectively, Table 4.5). Six wks treatment with $7.5 \mathrm{~g} A X$ or $15 \mathrm{~g} A X$ versus placebo did not change the Simpson evenness index.

\section{Microbiota composition and taxon distribution}

The PCoA analyses, based on the BC (dis)similarity matrix of the bacterial community sequence data, per test day are shown as Online Supplemental Material Figure S4.2, S4.3 and S4.4. In order to see if treatment during study period significantly altered fecal microbial composition, a two-way ANOSIM was performed. Two-way ANOSIM applying the $\mathrm{BC}$ coefficient showed no significant differences for treatment $(P=0.746)$ using time as factor. The heat map analysis showed that variations observed were not linked to a specific dosage of the test product and were masked by the interindividual variability among subjects. The cluster dendrogram showed that, with a few exceptions mainly related to the $15 \mathrm{~g} \mathrm{AX}$, samples from the same donor cluster together at baseline, after 3 wks intervention and end of the study, irrespective of the treatment received (data not shown). 
Table 4.5 Observed richness, Shannon diversity and Simpson evenness indices at baseline and after 6 wks supplementation.

\begin{tabular}{lcccccccc}
\hline Indices & Intervention & \multicolumn{2}{c}{ Baseline } & \multicolumn{2}{c}{ End } & Difference & $95 \% \mathrm{Cl}$ & $P$ value \\
& & Mean & SD & Mean & SD & in means & & \\
\hline Observed richness & Placebo & 295.3 & 21.4 & 285.2 & 44.9 & & & \\
& $7.5 \mathrm{~g} \mathrm{AX}$ & 301.4 & 49.3 & 287.8 & 33.1 & -1.9 & $-39.1,35.3$ & 0.918 \\
& $15 \mathrm{~g} \mathrm{AX}$ & 272.4 & 40.3 & 235.8 & 62.5 & -36.7 & $-69.3,-4.0$ & 0.029 \\
Shannon diversity & Placebo & 4.13 & 0.28 & 4.05 & 0.33 & & & \\
& $7.5 \mathrm{~g} \mathrm{AX}$ & 4.28 & 0.37 & 4.03 & 0.31 & -0.11 & $-0.41,0.20$ & 0.473 \\
& $15 \mathrm{~g} \mathrm{AX}$ & 4.08 & 0.25 & 3.75 & 0.43 & -0.29 & $-0.55-0.02$ & 0.036 \\
Simpson evenness & Placebo & 0.96 & 0.02 & 0.96 & 0.02 & & & \\
& $7.5 \mathrm{~g} \mathrm{AX}$ & 0.97 & 0.02 & 0.94 & 0.03 & -0.01 & $-0.04,0.01$ & 0.278 \\
& $15 \mathrm{~g} \mathrm{AX}$ & 0.96 & 0.01 & 0.94 & 0.03 & -0.02 & $-0.04,0.00$ & 0.063 \\
\hline
\end{tabular}

Differences between placebo, $7.5 \mathrm{~g} \mathrm{AX}$ and $15 \mathrm{~g}$ AX tested with linear mixed model. The presented difference in means is corrected for baseline differences. AX, arabinoxylans.

\section{Fecal pH, ammonium and SCFA concentrations}

Data regarding fecal $\mathrm{pH}$, ammonium and SCFA concentrations are depicted in Table 4.6. Fecal pH was significantly reduced after 6 wks $7.5 \mathrm{~g}$ AX supplementation, compared to placebo $(P=0.012)$. Six wks daily intake of both $7.5 \mathrm{~g}$ and $15 \mathrm{~g} A X$ increased the concentration of total SCFA, compared to a decrease in the placebo group, the difference being statistically significant (7.5 g AX: $P=0.012 ; 15 \mathrm{~g} \mathrm{AX}: P=0.006)$. The same was observed for fecal concentrations of acetate $(7.5 \mathrm{~g} A X: P=0.038 ; 15 \mathrm{~g} A X: P=0.016)$ and butyrate (7.5 g AX: $P=0.001 ; 15 \mathrm{~g} \mathrm{AX:} P=0.030$ ). Fecal propionate concentrations significantly increased in the $15 \mathrm{~g} A X$ group, compared to placebo $(P=0.026)$. $A X$ intake versus placebo did not affect fecal ammonium concentrations (7.5 g AX: $P=0.111 ; 15 \mathrm{~g} A X$ : $P=0.370$ ). No significant differences between interventions were seen in fecal $\mathrm{pH}$, ammonium and SCFA concentrations after 3 wks supplementation (all $P \geq 0.082$, data not shown).

\section{Production of cytokines by stimulated peripheral blood mononuclear cells}

Production of the pro-inflammatory cytokine TNFa by stimulated PBMCs was significantly reduced after 6 weeks $15 \mathrm{~g} A X$ intake $(P=0.035$; Table 4.7) compared to placebo. No significant differences were found regarding the production of the other measured cytokines (all $P \geq 0.176$; Table 4.7). 
Table $4.6 \quad F e c a l \mathrm{pH}$, ammonium (mg/l) and short-chain fatty acid concentrations ( $\mu \mathrm{mol} / \mathrm{g}$ feces) at baseline and after 6 wks supplementation.

\begin{tabular}{lcccc}
\hline & Intervention & Baseline & End & $P$ value \\
\hline $\mathrm{pH}$ & Placebo & $7.3[7.0 ; 7.6]$ & $7.7[7.3 ; 8.1]$ & \\
& $7.5 \mathrm{~g} \mathrm{AX}$ & $7.1[6.8 ; 7.5]$ & $7.0[6.9 ; 7.4]$ & 0.013 \\
& $15 \mathrm{~g} \mathrm{AX}$ & $7.2[6.9 ; 7.9]$ & $7.4[6.9 ; 7.7]$ & 0.086 \\
Ammonium & Placebo & $58.5[46.1 ; 74.5]$ & $44.8[34.7 ; 59.9]$ & \\
& $7.5 \mathrm{~g} \mathrm{AX}$ & $53.8[45.8 ; 69.7]$ & $55.4[41.9 ; 91.4]$ & 0.111 \\
& $15 \mathrm{~g} \mathrm{AX}$ & $43.4[28.2 ; 60.8]$ & $51.8[31.5 ; 88.1]$ & 0.370 \\
SCFA: total & Placebo & $67.4[46.4 ; 97.0]$ & $59.1[31.7 ; 63.8]$ & \\
& $7.5 \mathrm{~g} \mathrm{AX}$ & $63.6[50.0 ; 86.2]$ & $71.6[54.7 ; 88.3]$ & 0.012 \\
& $15 \mathrm{~g} \mathrm{AX}$ & $49.3[33.7 ; 72.5]$ & $59.2[41.8 ; 80.3]$ & 0.006 \\
SCFA: acetate & Placebo & $34.8[29.2 ; 49.9]$ & $31.2[17.4 ; 35.4]$ & \\
& $7.5 \mathrm{~g} \mathrm{AX}$ & $35.6[28.6 ; 44.7]$ & $35.0[25.4 ; 53.3]$ & 0.038 \\
& $15 \mathrm{~g} \mathrm{AX}$ & $27.8[17.9 ; 40.1]$ & $34.0[23.0 ; 44.5]$ & 0.016 \\
SCFA: propionate & Placebo & $11.1[7.7 ; 18.4]$ & $9.8[6.1 ; 12.2]$ & \\
& $7.5 \mathrm{~g} \mathrm{AX}$ & $11.5[8.7 ; 17.0]$ & $12.5[9.0 ; 15.5]$ & 0.229 \\
& $15 \mathrm{~g} \mathrm{AX}$ & $10.2[6.4 ; 17.0]$ & $12.7[8.4 ; 17.6]$ & 0.026 \\
SCFA: butyrate & Placebo & $9.0[6.1 ; 27.4]$ & $6.7[3.7 ; 9.9]$ & $11.9[9.4 ; 17.0]$ \\
& $7.5 \mathrm{~g} \mathrm{AX}$ & $10.9[8.7 ; 14.3]$ & $7.4[5.2 ; 14.6]$ & 0.001 \\
& $15 \mathrm{~g} \mathrm{AX}$ & $6.9[4.5 ; 15.2]$ & 0.030 \\
\hline
\end{tabular}

Differences between placebo, $7.5 \mathrm{~g} \mathrm{AX}$ and $15 \mathrm{~g}$ AX tested with linear mixed model with correction for baseline values. Data are given as median [IQR, i.e. Q1; Q3]. AX, arabinoxylans. SCFA, short-chain fatty acids.

Table 4.7 Cytokine production by stimulated PBMCs $(\mathrm{ng} / \mathrm{mL})$ at baseline and after 6 wks supplementation.

\begin{tabular}{|c|c|c|c|c|}
\hline Cytokine production & Intervention & Baseline & End & $P$ value \\
\hline \multirow[t]{3}{*}{$\mathrm{IFN} \gamma$} & Placebo & 29.8 [5.0; 75.5] & 48.8 [12.6; 419.2] & \\
\hline & $7.5 \mathrm{~g} \mathrm{AX}$ & 14.2 [9.3; 59.3] & 38.7 [2.5; 104.5] & 0.176 \\
\hline & $15 \mathrm{gAX}$ & 54.9 [26.5; 85.3] & 49.5 [10.7; 87.7] & 0.211 \\
\hline \multirow[t]{3}{*}{$\|-2$} & Placebo & 15.2 [0.9; 38.2] & 7.5 [1.2; 23.6] & \\
\hline & $7.5 \mathrm{~g} \mathrm{AX}$ & $0.3[0.1 ; 7.3]$ & $5.3[0.2 ; 41.1]$ & 0.427 \\
\hline & $15 \mathrm{~g} \mathrm{AX}$ & $10.7[0.4 ; 53.4]$ & $1.5[0.2 ; 49.9]$ & 0.489 \\
\hline \multirow[t]{3}{*}{$11-10$} & Placebo & $6.7[2.6 ; 18.6]$ & 12.8 [ $11.4 ; 27.1]$ & \\
\hline & $7.5 \mathrm{~g} \mathrm{AX}$ & $3.8[1.5 ; 10.1]$ & 5.3 [3.2; 18.4] & 0.205 \\
\hline & $15 \mathrm{~g} \mathrm{AX}$ & 8.3 [5.0; 18.3] & 11.5 [5.6; 17.2] & 0.402 \\
\hline \multirow[t]{3}{*}{$\|-12 p 40$} & Placebo & $0.02[0.01 ; 0.16]$ & 0.05 [0.02; 0.17] & \\
\hline & $7.5 \mathrm{~g} \mathrm{AX}$ & 0.02 [0.01; 0.03] & 0.04 [0.02; 0.06] & 0.210 \\
\hline & $15 \mathrm{~g} \mathrm{AX}$ & 0.02 [0.01; 0.07] & 0.02 [0.00; 0.06] & 0.861 \\
\hline \multirow[t]{3}{*}{ TNFa } & Placebo & $72.9[44.5 ; 147.6]$ & 97.2 [52.4; 119.3$]$ & \\
\hline & $7.5 \mathrm{~g} \mathrm{AX}$ & 56.9 [28.6; 103.4] & 61.9 [34.0; 138.7] & 0.708 \\
\hline & $15 \mathrm{gAX}$ & $83.0[50.5 ; 96.0]$ & $60.9[27.4 ; 77.6]$ & 0.035 \\
\hline
\end{tabular}

Differences between placebo, $7.5 \mathrm{~g} \mathrm{AX}$ and $15 \mathrm{~g} A X$ tested with linear mixed model with correction for baseline values. Data are given as median [IQR, i.e. Q 1; Q3]. AX, arabinoxylans. IFN, interferon. TNF, tumor necrosis factor.

\section{Blood lipids, glucose and insulin}

At baseline, total cholesterol, LDL- and HDL-cholesterol, triglycerides, free fatty acids, glucose and insulin lied within normal blood value ranges. These parameters were not 
significantly altered by either 3 wks or 6 wks supplementation with $7.5 \mathrm{~g}$ or $15 \mathrm{~g} \mathrm{AX}$ compared to placebo (all $P \geq 0.083$; Table 4.8). Subsequently, no change between intervention groups was observed regarding insulin sensitivity (all $P \geq 0.768$, Table 4.8).

Table 4.8 Blood lipids (mmol/l), glucose $(\mathrm{mmol} / \mathrm{I})$, insulin $(\mathrm{pmol} / \mathrm{l})$ and QUICKI at baseline and after 6 wks supplementation.

\begin{tabular}{|c|c|c|c|c|c|c|c|c|}
\hline \multirow[t]{2}{*}{ Metabolic markers } & \multirow[t]{2}{*}{ Intervention } & \multicolumn{2}{|c|}{ Baseline } & \multicolumn{2}{|c|}{ End } & \multirow{2}{*}{$\begin{array}{l}\text { Difference } \\
\text { in means }\end{array}$} & \multirow[t]{2}{*}{$95 \% \mathrm{Cl}$} & \multirow[t]{2}{*}{$P$ value } \\
\hline & & Mean & SD & Mean & SD & & & \\
\hline \multirow[t]{3}{*}{ Total cholesterol } & Placebo & 5.39 & 1.00 & 5.37 & 1.24 & & & \\
\hline & $7.5 \mathrm{~g} \mathrm{AX}$ & 5.83 & 1.00 & 5.71 & 1.05 & -0.11 & $-0.49,0.27$ & 0.563 \\
\hline & $15 \mathrm{gAX}$ & 5.59 & 0.80 & 5.46 & 0.99 & -0.12 & $-0.49,0.25$ & 0.524 \\
\hline \multirow[t]{3}{*}{ LDL-C } & Placebo & 3.26 & 0.77 & 3.29 & 0.95 & & & \\
\hline & $7.5 \mathrm{~g} \mathrm{AX}$ & 3.67 & 0.83 & 3.56 & 0.99 & -0.14 & $-0.52,0.23$ & 0.442 \\
\hline & $15 \mathrm{gAX}$ & 3.39 & 0.75 & 3.16 & 0.90 & -0.26 & $-0.62,0.10$ & 0.148 \\
\hline \multirow[t]{3}{*}{ HDL-C } & Placebo & 1.42 & 0.31 & 1.46 & 0.35 & & & \\
\hline & $7.5 \mathrm{~g} \mathrm{AX}$ & 1.44 & 0.41 & 1.41 & 0.41 & -0.06 & $-0.20,0.08$ & 0.357 \\
\hline & $15 \mathrm{gAX}$ & 1.60 & 0.36 & 1.64 & 0.45 & 0.00 & $-0.14,0.13$ & 0.972 \\
\hline \multirow[t]{3}{*}{ Triglycerides } & Placebo & 1.57 & 0.58 & 1.37 & 0.51 & & & \\
\hline & $7.5 \mathrm{~g} \mathrm{AX}$ & 1.79 & 1.32 & 2.06 & 2.11 & 0.41 & $-0.08,0.90$ & 0.102 \\
\hline & $15 \mathrm{gAX}$ & 1.32 & 0.64 & 1.47 & 0.82 & 0.42 & $-0.06,0.89$ & 0.083 \\
\hline \multirow[t]{3}{*}{ Glucose } & Placebo & 5.38 & 0.49 & 5.39 & 0.66 & & & \\
\hline & $7.5 \mathrm{~g} \mathrm{AX}$ & 5.54 & 0.90 & 5.36 & 0.50 & -0.08 & $-0.45,0.28$ & 0.659 \\
\hline & $15 \mathrm{gAX}$ & 5.26 & 0.54 & 5.12 & 0.38 & -0.24 & $-0.59,0.12$ & 0.186 \\
\hline \multirow[t]{3}{*}{ Insulin } & Placebo & 75.8 & 29.8 & 62.4 & 26.3 & & & \\
\hline & $7.5 \mathrm{~g} \mathrm{AX}$ & 124.8 & 250.5 & 68.3 & 33.9 & 3.74 & $-25.4,32.9$ & 0.797 \\
\hline & $15 \mathrm{gAX}$ & 70.7 & 35.9 & 68.4 & 48.7 & 6.26 & $-22.4,34.9$ & 0.661 \\
\hline \multirow[t]{3}{*}{ QUICKI } & Placebo & 0.26 & 0.01 & 0.27 & 0.02 & & & \\
\hline & $7.5 \mathrm{~g} \mathrm{AX}$ & 0.26 & 0.02 & 0.27 & 0.02 & -0.0018 & $-0.01,0.01$ & 0.768 \\
\hline & $15 \mathrm{gAX}$ & 0.27 & 0.02 & 0.27 & 0.02 & 0.0003 & $-0.01,0.01$ & 0.963 \\
\hline
\end{tabular}

Differences between placebo, $7.5 \mathrm{~g} A X$ and $15 \mathrm{~g} A X$ tested with linear mixed model. The presented difference in means is corrected for baseline differences. AX, arabinoxylans. QUICKI, quantitative insulin sensitivity check index.

\section{Gastrointestinal tolerance}

No significant differences between intervention groups regarding reported $G$ symptoms were observed during the study (all $P \geq 0.118$, data not shown). Mean scores remained under 2 during all treatments, which means that the participants during intervention experienced no troublesome symptoms. Neither $7.5 \mathrm{~g}$ nor $15 \mathrm{~g}$ AX significantly affected stool frequency or consistency throughout the entire study period lall $P \geq 0.271$, data not shown). 


\section{Discussion}

In this study, we have shown that Gl permeability is compromised in overweight and obese individuals when compared to lean controls. Six wks intake of $7.5 \mathrm{~g}$ or $15 \mathrm{~g}$ AX per day did not affect $G$ permeability, as assessed by the multi-sugar test. Although no differences were found in the relative abundance of the dominant phyla, intake of $15 \mathrm{~g}$ AX resulted in reduced microbial richness and diversity, when compared to placebo. AX supplementation increased fecal SCFA concentrations. Furthermore, $7.5 \mathrm{~g} \mathrm{AX}$ intake significantly lowered fecal $\mathrm{pH}$. This modulation of the intestinal luminal environment may positively affect the gut barrier, as reflected by upregulation in the transcription of different TJ proteins in sigmoid biopsies, although we did not detect significant changes in TJ protein expression. The decreased TNFa production by stimulated PBMCs in the high dose (15 g) AX group points to downstream positive anti-inflammatory effects.

Evidence indicating that obesity is associated with an impaired gut barrier is mainly derived from animal models. ${ }^{3}$ Human studies on gut barrier and obesity are scarce, and results contradictory. ${ }^{22,23}$ Here, we showed that gut permeability is increased in obese compared to lean healthy volunteers. AX treatment did not affect gastroduodenal, small intestinal, colonic or whole gut permeability, as assessed with a multi-sugar test. However, interpretation of these results must be done with caution as permeability testing with sugars might be influenced by individual differences in gastric emptying and intestinal transit time, parameters we did not measure in these participants. Furthermore, although we aimed to examine a homogenous study population, possible confounders (i.e. lifestyle, sex) could have affected permeability results. Prebiotics, such as inulin, were shown to improve Gl permeability in obese subjects after dietary intervention for 9 wks. ${ }^{24}$ The discrepancy in results between our and above study might be explained by various factors, such as differences in method of measuring Gl permeability, sample size and duration of study product intake. Furthermore, we assessed the effects of $A X$ intake during weight maintenance, while they investigated the effect during a weight loss intervention. ${ }^{24}$ Some animal studies have shown beneficial effects of $A X$ on an impaired gut barrier. 9.10 This is the first human study analysing the effects of a (putative) prebiotic on the expression of TJ proteins in sigmoid mucosa in overweight subjects. From a subset of our study population we obtained mucosal samples from sigmoid colon and despite the small sample size, $7.5 \mathrm{~g} \mathrm{AX}$ showed to significantly upregulate gene transcription of the TJ protein occludin, while $15 \mathrm{~g}$ AX significantly upregulated claudin-3 and claudin-4. These proteins are structural transmembrane components, which organize complex TJ protein systems, determining paracellular permeability. These results are in line with the preclinical data in obese mice, where AX significantly increased mRNA concentrations of TJ proteins expression. ${ }^{9}$ No significant changes in sigmoid TJ protein concentrations were observed after $A X$ treatment. 
Modulating gut microbiota composition by prebiotic compounds, and thereby influencing its effect on intestinal homeostasis, appears to be a promising strategy to treat and prevent chronic diseases. This is the first human study investigating the effect of a high molecular weight $A X$ on the microbial community composition in overweight and obese individuals. In vitro and mice studies have shown that long-chain AX can be efficiently fermented, leading to specific stimulation of bifidobacterial species and beneficial fermentation profiles. 911 While in previous studies the effect of this particular AX concentrate on the microbiota was assessed by selective 9PCR protocols, ${ }^{10-12}$ in the current trial we made use of a metagenomic approach. Here, we observed a decrease in microbial richness and diversity after $15 \mathrm{~g}$ AX supplementation, but no change in overall microbiota composition was seen. Intestinal microbiota converts indigestible food components into metabolites, such as SCFA, that can be processed by the host. SCFA are able to exert antioxidant, anti-inflammatory and immunomodulating effects. ${ }^{25}$ Furthermore, the SCFA butyrate functions as the principal energy source for the proliferation and differentiation of colonic epithelial cells and is essential in establishing and maintaining the gut barrier by regulating the expression of TJ proteins. ${ }^{25}$ We demonstrated that the fecal concentration of the individual and total SCFA were increased upon intake of $A X$ compared to a decrease in the placebo group. Our findings confirm previous data from in vitro and in vivo studies. Two different in vitro models, often used to assess modulation of the gut microbiome (SHIME, TIM-2), demonstrated that long-chain AX specifically increase propionate concentrations. ${ }^{11}$ In germ-free rats, inoculated with human feces, long-chain AX increased the concentrations of acetate, propionate, butyrate and total amount of SCFA. ${ }^{12}$ In a human study, medium-chain AX significantly increased fecal concentrations of both propionic and butyric acid after 4 wks intake. $^{26}$ Other prebiotics, such as inulin and fructo-oligosaccharides, showed less pronounced effects on SCFA production in humans. ${ }^{27,28}$ Concomitantly, we found a significant decrease in fecal $\mathrm{pH}$ in the $7.5 \mathrm{~g}$ AX group. A more acidic luminal environment may contribute to the reduction of pathogenic bacteria and creates a more favourable environment for the growth of beneficial bacteria.

To assess the effect of $A X$ on immune response we applied an ex vivo model using PHAstimulated whole blood to evaluate the capacity to produce cytokines. We found a significant decrease in production of the pro-inflammatory cytokine TNFa after supplementation with $15 \mathrm{~g} \mathrm{AX}$, suggesting a capacity to reduce the intensity of an acute pro-inflammatory reaction. In obese mice $A X$ showed to have an anti-inflammatory effect. Human data regarding the effect of prebiotics on immune modulation, assessed via the current ex vivo model, are limited. One study in healthy subjects showed that a prebiotic mixture had the capacity to reduce the pro-inflammatory pathway and to stimulate antiinflammatory pathways. ${ }^{26}$ Pro-inflammatory cytokines are associated with the development of systemic IR, B-cell dysfunction and CVD. ${ }^{29}$ One important function of SCFA is suppressing the production of such cytokines. ${ }^{25}$ In the present human trial we observed an increase in 
fecal SCFA concentrations after $A X$ treatment, providing a possible explanation for the decrease in TNFa production by PBMC.

Obesity is typically associated with a wide cluster of metabolic alterations, including glucose homeostasis disorders and dyslipidemia. AX showed to improve glycemic control and blood triglycerides concentrations in subjects with an impaired glucose tolerance or DM2. ${ }^{29}$ In the present study, supplementation with $A X$ in healthy overweight and obese subjects did not affect systemic glucose, insulin and lipid concentrations. This can be explained by the fact that the participants had normal values from beginning of the study.

The daily ingestion of $A X$ did not influence bowel habits, confirming previous observations in prebiotics intervention studies. ${ }^{28}$ The low prevalence of $G$ complaints during $A X$ supplementation provides evidence that $A X$ is well-tolerated by humans.

This study followed a unique approach to assess the gut barrier, by combining functional analyses (multi-sugar test) with TJ analyses in mucosal tissue specimens. Some potential shortcomings should be considered. Firstly, we measured fecal SCFA concentrations to assess the microbiota fermentation capacity. SCFA are efficiently absorbed from the gut, with only $5 \%$ to $10 \%$ being excreted via the feces. Fecal SCFA concentrations hence do not accurately reflect the exact intestinal SCFA concentration. However, previous studies showed that the ratio between the respective SCFA, measured in feces and colon, is comparable to each other. ${ }^{31}$ Thus, changes in fecal SCFA concentration reflect relative changes in intestinal concentrations of the separate SCFA. Furthermore, as we choose to focus on the effects of $A X$ on gut barrier function and gut microbiota, we did not obtain information regarding hepatic health of our study subjects. Prebiotics target multiple metabolic impairments associated with obesity-related non-alcohol fatty liver disease, and thus the effects of $A X$ on hepatic health in this study population may be another interesting objective to assess. We did not control the diet and exercise pattern of our participants, as we aimed to evaluate the effects of $A X$ when supplementing the habitual diet, which is in line with future applications. Furthermore, in this study we observed potentially advantageous findings regarding gut barrier and immune function, however these findings are still theoretical and were not translated into clinically positive outcomes. Future research is required to assess whether these findings can improve gut barrier function and immune health.

In conclusion, we have shown that 6 wks AX supplementation resulted in a more beneficial fermentation (SCFA) profile in overweight and obese subjects. In addition, an increased gene transcription of several transmembrane proteins was observed, although no changes in TJ proteins expressions or Gl permeability were detected. Furthermore, we showed that $15 \mathrm{~g} \mathrm{AX}$ might have an anti-inflammatory potential and that the intake of $A X$ was welltolerated. 


\section{References}

1. Viggiano D, laniro G, Vanella $G$, et al. Gut barrier in health and disease: focus on childhood. Eur Rev Med Pharmacol Sci. 2015; 19(6): 1077-85.

2. Guinane CM, Cotter PD. Role of the gut microbiota in health and chronic gastrointestinal disease: understanding a hidden metabolic organ. Therap Adv Gastroenterol. 2013;6(4):295-308.

3. Cani PD, Possemiers $S$, Van de Wiele T, et al. Changes in gut microbiota control inflammation in obese mice through a mechanism involving GLP-2-driven improvement of gut permeability. Gut 2009:58(8): 1091-103.

4. Hotamisligil GS, Erbay E. Nutrient sensing and inflammation in metabolic diseases. Nat Rev Immunol. 2008:8(12):923-34.

5. Gibson GR, Roberfroid MB. Dietary modulation of the human colonic microbiota: introducing the concept of prebiotics. J Nutr. 1995; 125(6): 1401-12.

6. Grootaert C, Van den Abbeele P, Marzorati M, et al. Comparison of prebiotic effects of arabinoxylan oligosaccharides and inulin in a simulator of the human intestinal microbial ecosystem. FEMS Microbiol Ecol 2009:69(2):231-42.

7. Hughes SA, Shewry PR, Li L, et al. In vitro fermentation by human fecal microflora of wheat arabinoxylans. J Agric Food Chem. 2007:55(1 1):4589-95.

8. Monobe M, Ema K, Kato F, Maeda-Yamamoto M. Immunostimulating activity of a crude polysaccharide derived from green tea (Camellia sinensis) extract. J Agric Food Chem. 2008;56(4): 1423-7.

9. Neyrinck AM, Possemiers S, Druart $C$, et al. Prebiotic effects of wheat arabinoxylan related to the increase in bifidobacteria, Roseburia and Bacteroides/Prevotella in diet-induced obese mice. PloS One $2011 ; 6(6): e 20944$

10. Chen H, Wang W, Degroote J, et al. Arabinoxylan in wheat is more responsible than cellulose for promoting intestinal barrier function in weaned male piglets. J Nutr. 2015; 145(1):51-8.

11. Van den Abbeele P, Venema K, Van de Wiele $T$, et al. Different human gut models reveal the distinct fermentation patterns of Arabinoxylan versus inulin. J Agric Food Chem. 2013;61(41):9819-27.

12. Van den Abbeele P, Gerard P, Rabot S, et al. Arabinoxylans and inulin differentially modulate the mucosal and luminal gut microbiota and mucin-degradation in humanized rats. Environ Microbiol 201 1;13(10): 266780.

13. Mujagic $Z$, Ludidi $S$, Keszthelyi $D$, et al. Small intestinal permeability is increased in diarrhoea predominant IBS, while alterations in gastroduodenal permeability in all IBS subtypes are largely attributable to confounders. Aliment Pharmacol Ther. 2014;40(3):288-97.

14. van Wiick K, van Eijk HM, Buurman WA, et al. Novel analytical approach to a multi-sugar whole gut permeability assay. J Chromatogr B Analył Technol Biomed Life Sci. 201 1;879(26): 2794-801.

15. Piils $\mathrm{KE}$, Koek $\mathrm{GH}$, Elamin $\mathrm{EE}$, et al. Large intestine permeability is increased in patients with compensated liver cirrhosis. Am J Physiol Gastrointest Liver Physiol. 2014; 306(2):G147-53.

16. Keszthelyi D, Troost FJ, Jonkers DM, et al. Serotonergic reinforcement of intestinal barrier function is impaired in irritable bowel syndrome. Aliment Pharmacol Ther. 2014;40(4):392-402.

17. Verstraelen H, Vilchez-Vargas R, Desimpel F, et al. Characterisation of the human uterine microbiome in nonpregnant women through deep sequencing of the V1-2 region of the 16S rRNA gene. PeerJ 2016;4:e1602.

18. Possemiers S, Verthe K, Uyttendaele S, Verstraete W. PCR-DGGE-based quantification of stability of the microbial community in a simulator of the human intestinal microbial ecosystem. FEMS Microbiol Ecol. 2004;49(3):495-507.

19. Bremner JMaK, R.D. Steam distillation methods for determination of ammonium, nitrate and nitrite. Anal Chem Acta. 1965:32:485-95.

20. Svedlund J, Sjodin I, Dotevall G. GSRS - a clinical rating scale for gastrointestinal symptoms in patients with irritable bowel syndrome and peptic ulcer disease. Dig Dis Sci. 1988;33(2):129-34. 
21. Kim H, Bartley GE, Young SA, et al. HPMC supplementation reduces abdominal fat content, intestinal permeability, inflammation, and insulin resistance in diet-induced obese mice. Mol Nutr Food Res. 2012;56(9): $1464-76$

22. Teixeira TF, Souza NC, Chiarello PG, et al. Intestinal permeability parameters in obese patients are correlated with metabolic syndrome risk factors. Clin Nutr. 2012;31(5):735-40.

23. Brignardello J, Morales P, Diaz E, et al. Pilot study: alterations of intestinal microbiota in obese humans are not associated with colonic inflammation or disturbances of barrier function. Aliment Pharmacol Ther. 2010:32(1 1-12): 1307-14.

24. Xiao S, Fei N, Pang $X$, et al. A gut microbiota-targeted dietary intervention for amelioration of chronic inflammation underlying metabolic syndrome. FEMS Microbiol Ecol. 20 14;87(2):357-67.

25. Canani RB, Costanzo MD, Leone $L$, et al. Potential beneficial effects of butyrate in intestinal and extraintestinal diseases. World J Gastroenterol. 2011 ; 17(12): 1519-28.

26. Lecerf JM, Depeint F, Clerc E, et al. Xylo-oligosaccharide (XOS) in combination with inulin modulates both the intestinal environment and immune status in healthy subjects, while XOS alone only shows prebiotic properties. Br J Nutr. 2012;108(10): 1847-58.

27. van Dokkum W, Wezendonk B, Srikumar TS, van den Heuvel EG. Effect of nondigestible oligosaccharides on large-bowel functions, blood lipid concentrations and glucose absorption in young healthy male subjects. Eur J Clin Nutr. 1999;53(1): 1-7.

28. Slavin J, Feirtag J. Chicory inulin does not increase stool weight or speed up intestinal transit time in healthy male subjects. Food Funct. 201 1;2(1):72-7.

29. Goldberg RB. Cytokine and cytokine-like inflammation markers, endothelial dysfunction, and imbalanced coagulation in development of diabetes and its complications. J Clin Endocrinol Metab. 2009:94(9):317182.

30. Lu ZX, Walker KZ, Muir JG, O'Dea K. Arabinoxylan fibre improves metabolic control in people with Type II diabetes. Eur J Clin Nutr. 2004;58(4):621-8.

31. Macfarlane G.T. GG. Microbiological aspects of production of short-chain fatty acids in the large bowel. Cambridge: Cambridge University Press, 1999. 
Supplemental material

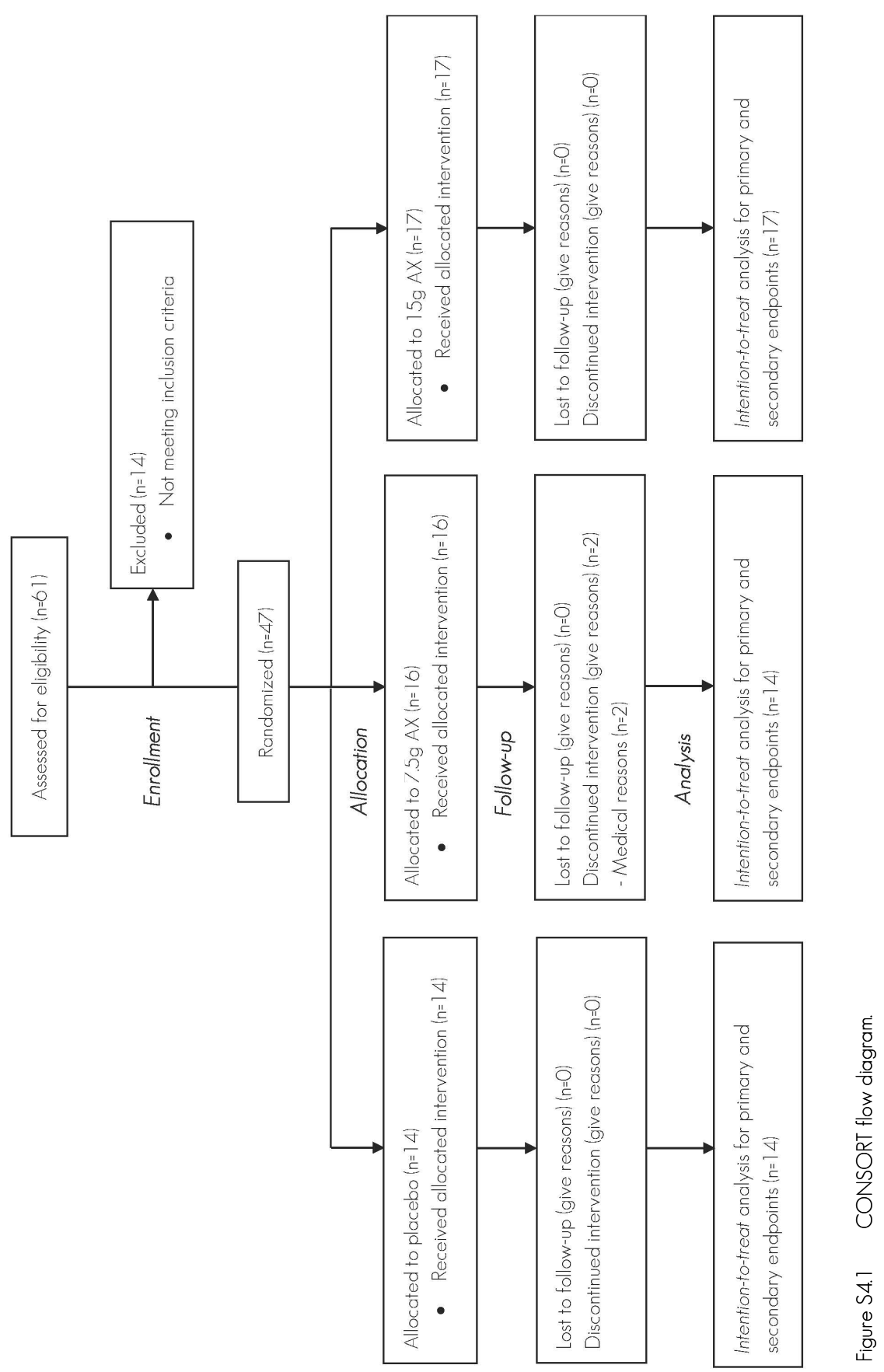




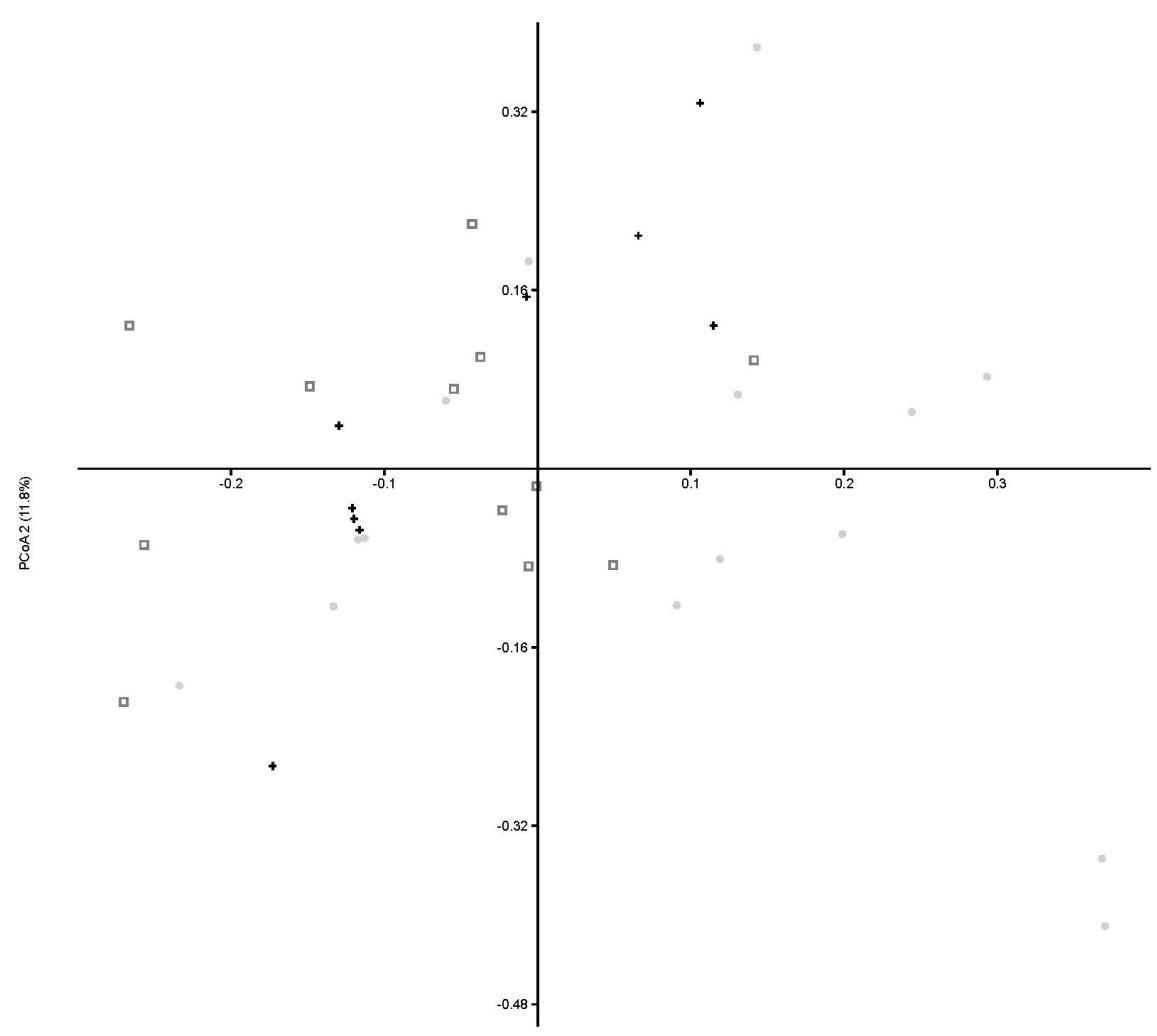

PCOA $1(21.8 \%)$

Figure S4.2 Clustering of samples due to intervention by PCoA, based on BC similarity distance, at baseline. Fecal samples collected from different treatment groups are represented by gray squares for placebo, black plusses for $7.5 \mathrm{~g}$ AX and lightgray dots for $15 \mathrm{~g}$ AX. No significant differences in microbial community composition between placebo, $7.5 \mathrm{~g}$ AX and $15 \mathrm{~g}$ AX during study period were observed, as assessed using two-way ANOSIM. ANOSIM, analysis of similarity. AX, arabinoxylans. BC, Bray-Curtis. PCoA, principle coordinate analysis. 


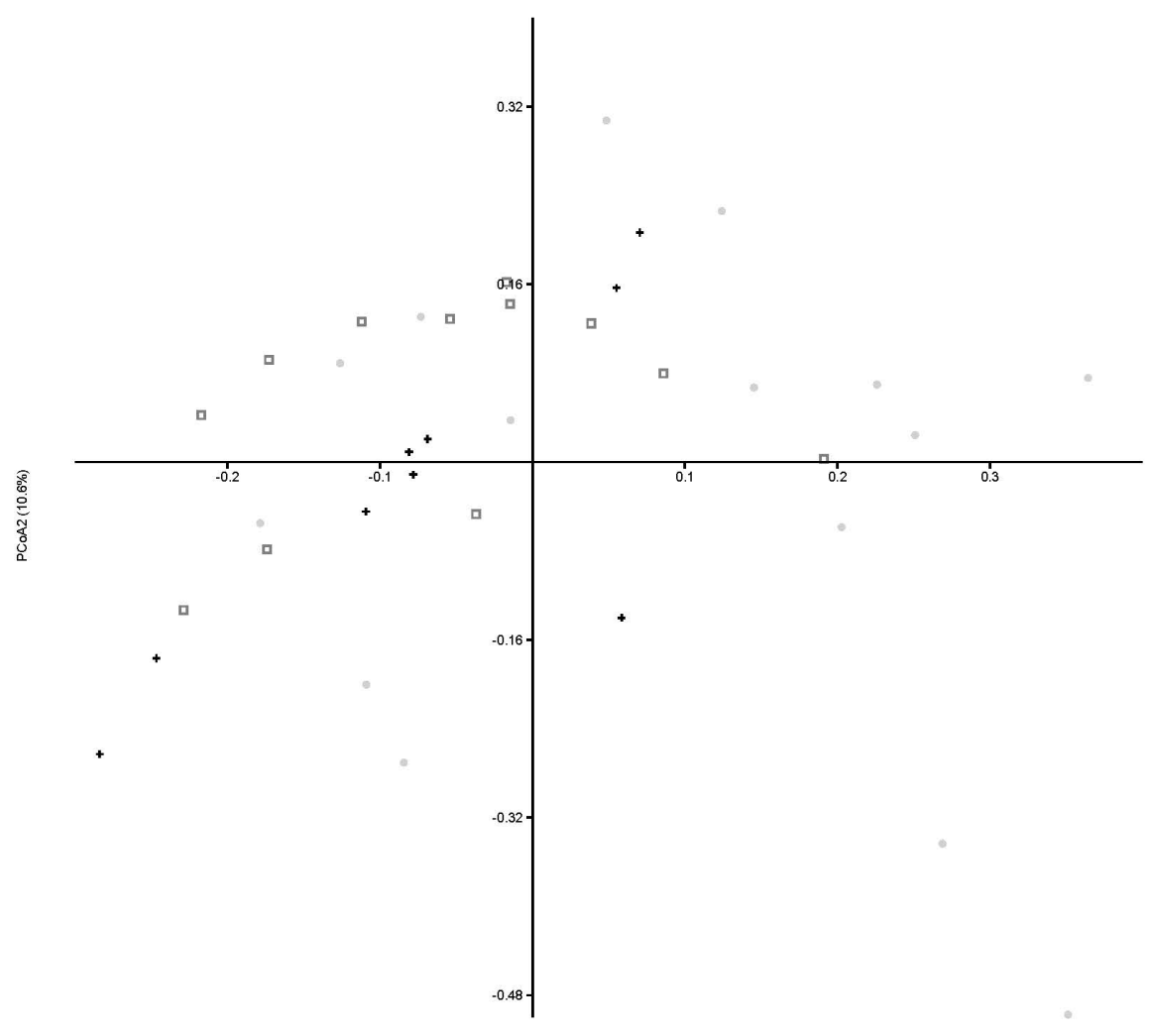

PCOA1 $(22.1 \%)$

Figure S4.3 Clustering of samples due to intervention by PCoA, based on BC similarity distance, after 3 wks intervention. Fecal samples collected from different treatment groups are represented by gray squares for placebo, black plusses for $7.5 \mathrm{~g} \mathrm{AX}$ and lightgray dots for $15 \mathrm{~g} \mathrm{AX}$. No significant differences in microbial community composition between placebo, $7.5 \mathrm{~g} A X$ and $15 \mathrm{~g} \mathrm{AX}$ during study period were observed, as assessed using two-way ANOSIM. ANOSIM, analysis of similarity. AX, arabinoxylans. BC, Bray-Curtis. PCoA, principle coordinate analysis. 


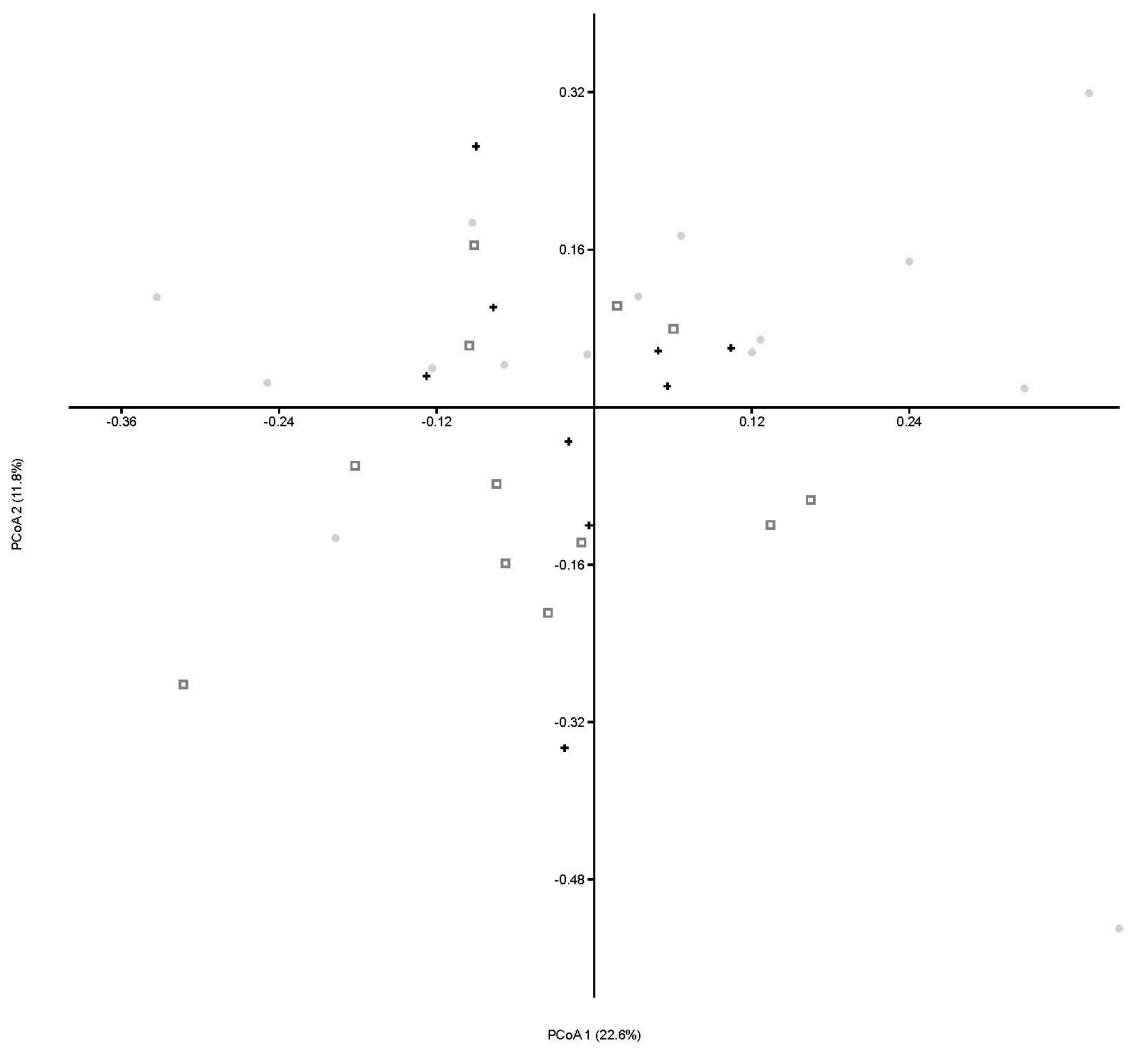

Figure S4.4 Clustering of samples due to intervention by PCoA, based on BC similarity distance, after 6 wks intervention. Fecal samples collected from different treatment groups are represented by gray squares for placebo, black plusses for $7.5 \mathrm{~g}$ AX and light gray dots for $15 \mathrm{~g} \mathrm{AX}$. No significant differences in microbial community composition between placebo, $7.5 \mathrm{~g} \mathrm{AX}$ and $15 \mathrm{~g} \mathrm{AX}$ during study period were observed, as assessed using two-way ANOSIM. ANOSIM, analysis of similarity. AX, arabinoxylans. BC, Bray-Curtis. PCoA, principle coordinate analysis. 
$\nabla m$ 


\section{chapter 5}

In vivo bioavailability of hesperidin $2 \mathrm{~S}$ is improved by micronization: a randomized, double-blind cross-over study

Salden BN, Troost FJ, Possemiers S, Bast A, Masclee AA 


\section{Abstract}

\section{Background}

The bioavailability of hesperidin in humans is low, partly due to its poor water solubility. Micronization is known to decrease particle size of a compound, thereby improving its solubility and thus absorption.

\section{Objective}

To investigate the bioavailability of a single dosage micronized hesperidin $2 S$ compared to an identical dosage standard non-micronized hesperidin in healthy individuals.

\section{Design}

In this double-blind, randomized, crossover exploratory study 11 healthy individuals attended to two test days. Subjects randomly consumed one dosage standard non-micronized hesperidin and one dosage micronized hesperidin 2S. Urine was collected for $24 \mathrm{~h}$. Blood was sampled at 8 different time points over a $10 \mathrm{~h}$ period.

\section{Results}

LC-MS/MS analysis of urine and plasma samples revealed the metabolites hesperetin-3-Oglucuronide, hesperetin-7-O-glucuronide and hesperetin-7-O-sulphate. The cumulative and relative excretion of total hesperetin $(P=0.032$ ) and individual metabolites (all $P \leq 0.044$ ) in $24 \mathrm{~h}$ was significantly higher after micronized hesperidin $2 S$ intake, compared to standard hesperidin. Also the plasma hesperetin $\mathrm{AUC}_{0-4 \mathrm{~h}}$ was higher after intake of the micronized product, compared to nonmicronized product $(P=0.032$.

\section{Conclusions}

The bioavailability of micronized hesperidin $2 \mathrm{~S}$ was higher than standard non-micronized hesperidin. This finding may beneficially influence the biological activity and systemic health effects of this formulation. 


\section{Introduction}

Flavonoids comprise a large group of polyphenolic compounds present in fruits and vegetables. Several large studies have shown that the intake of flavonoid-rich foods has positive effects on cardiovascular diseases (CVD) and their related risk factors. ${ }^{1,2}$ Hesperidin (HE; hesperetin-7-O-rutinose 2S), a naturally extracted and purified flavanone glycoside from sweet orange peels, has drawn attention due to its positive effects on metabolic and cardiovascular health. ${ }^{3-6}$ In order to exert health effects in vivo, it is essential that flavonoids are bioavailable, absorbed from the gastrointestinal (Gl) tract and reach the systemic circulation. Following oral administration, hesperidin is absorbed across the $\mathrm{Gl}$ tract, but its bioavailability is low in humans. ${ }^{7.8}$ Several factors limit the bioavailability of hesperidin after oral intake, one of them is its poor water solubility. Furthermore, for many flavonoid glycosides, after conversion to its aglycone, the small intestine is the major site of absorption. ${ }^{10}$ However, hesperidin is a flavonoid consisting of a flavonoid backbone (hesperetin) bound to rutinose. To release and enable the absorption of the hesperidin aglycone, hydrolysis of rutinose by $\beta$-glucosidase derived from gut microbiota is required." This process mainly occurs in the large intestine, where the aglycone hesperetin is released and further conjugated into glucuronidated and sulphated metabolites by enterocyte cells or by the liver, these metabolites may then be absorbed into the blood. 8.12 As a result, circulating levels of hesperetin are very low: hesperetin sulfates (3'- and 7-O-sulfate) and glucuronides (3'- and 7-O-glucuronide) are the main circulating metabolites upon hesperidin consumption.7.,13 An additional complex factor is related to the specific enantiomer configuration of hesperidin (i.e. S versus R). Hesperidin consists of a mixture of $S$ - and R-enantiomers, it has a $S: R$ ratio between $1: 1$ and $5: 1$, depending on its source. ${ }^{14,15}$ While in nature the 2S-enantiomer of hesperidin, and subsequently the S-hesperetin enantiomer, is dominant, commercially available hesperidin and hesperetin are a mixture of both stereoisomers. The two enantiomers may display distinct kinetic and dynamic properties, with stronger biological effects elicited by the S-enantiomer compared to the R-enantiomer. ${ }^{16,17}$ Several attempts have been made to improve the bioavailability and subsequent bioactivity of hesperidin. ${ }^{18-20}$ Here, we developed a micronized hesperidin $2 S$ formulation with a S:R ratio of 4:1 (Cordiart). Micronization has previously been used to increase the bioavailability of other polyphenols. ${ }^{21}$ The particle size of the micronized hesperidin $2 S$ is approximately 10 times smaller than the standard hesperidin extract.

Primary aim of this study was to investigate the bioavailability of a single dose of micronized hesperidin 2S, as measured by $24 \mathrm{~h}$ urinary excretion and plasma AUC of hesperidin metabolites, compared to a standard non-micronized hesperidin formulation (S:R ratio of 1.5:1). We hypothesized that micronized hesperidin $2 S$ has an improved bioavailability compared to a standard non-micronized hesperidin product, as shown by a higher $24 \mathrm{~h}$ urinary excretion and plasma AUC of hesperidin metabolites. 


\section{Subjects and methods}

The study was approved by the Medical Ethics Committee of the Maastricht University Medical Center + (MUMC+) and conducted in full accordance with the principles of the Declaration of Helsinki of 1975 as amended in 2013 and with the Dutch Regulations on Medical Research involving Human Subjects (WMO, 1998). The study was performed at the MUMC+ in June and July 2013. All participants gave written informed consent before participation.

\section{Subjects}

Healthy volunteers aged $18-75$ years with a BMI between $18-30 \mathrm{~kg} / \mathrm{m}^{2}$ and normal laboratory values were recruited by advertising in the local media. Laboratory values included haemoglobin ( $\mathrm{Hb}$, defined as: M 8.2- $11.0 \mathrm{mmol} / \mathrm{l}, \mathrm{F}$ 7.3-9.7 mmol//); haematocrit ( $\mathrm{Ht}$, defined as: M 0.41-0.52 I/I, F 0.36-0.48 I/I); serum alanine transaminase (ALT, defined as: $M<45 \mathrm{U} / \mathrm{l}, \mathrm{F}<34 \mathrm{U} / \mathrm{l}$ ); serum aspartate transaminase (AST, defined as: $M<35 \mathrm{U} / \mathrm{l}$, $\mathrm{F}<31 \mathrm{U} /$ /); gamma-glutamyl transferase (GGT, defined as: $M<55 \mathrm{U} / \mathrm{l}, \mathrm{F}<40 \mathrm{U} / \mathrm{l}$ ); bilirubin total (defined as: $<20 \mu \mathrm{mol} / \mathrm{l}$ ); serum creatinine (defined as: $M$ 60-115 $\mu \mathrm{mol} / \mathrm{l}$, F 50- $100 \mu \mathrm{mol} / \mathrm{l}$ ). Exclusion criteria were: any medical condition that might interfere with the study and might jeopardize the health status of the participant; smoking; use of medication / vitamin-, mineral-, or antioxidant supplements; pregnancy and lactation; history of any side effects towards the intake of flavonoids or citrus fruits; failure to comply with prohibited intake of hesperidin rich food products.

\section{Design and intervention}

In this double-blind, randomized, crossover exploratory study each subject attended to two test days with at least 1 week washout period in between. All participants and investigators remained blind to treatment until all analyses were completed. Participants were requested to abstain from strenuous physical exercise and consumption of alcohol the day prior to each test day. Furthermore, we instructed them to maintain their habitual diet during the study period, but to abstain from intake of hesperidin-rich foods starting 3 days prior to start of the study period. After an overnight fast, subjects arrived at the study site and an intravenous catheter was inserted into the antecubital vein of the arm. Blood sampling was performed at baseline and $15 \mathrm{~min}, 30 \mathrm{~min}, 1 \mathrm{~h}, 2 \mathrm{~h}, 3 \mathrm{~h}, 4 \mathrm{~h}$ and $10 \mathrm{~h}$ after intake of the study product. Furthermore, after intake of the study product, subjects collected full urine output for $24 \mathrm{~h}$. One hour after intake of the study product, the participants received a standard breakfast. During a test day they also received a standard snack, lunch and dinner. At the end of the test day the intravenous catheter was removed. 


\section{Micronized hesperidin $2 \mathrm{~S}$ and standard hesperidin}

Micronized hesperidin 2S (450 mg, supplied as 500 mg Cordiart, BioActor BV, Maastricht, The Netherlands) was extracted from the Citrus sinesis immature fruits, containing both the $S$ and $R$ enantiomers in the natural 4:1 S:R ratio of hesperidin. Standard hesperidin (450 mg) was provided by Ferrer HealthTech (Spain) and had a S:R molar ratio of 1.5:1. A total of $450 \mathrm{mg}$ hesperidin corresponds to $222.8 \mathrm{mg}$ hesperetin. The study products were formulated into capsules, each containing $250 \mathrm{mg}$ study product. Subjects were asked to ingest two capsules within 1 min with $200 \mathrm{ml}$ water.

\section{Urine collection}

Twenty-four hour urine was collected in $2 \mathrm{~L}$ bottles with added dibutylhydroxytolvene lantioxidant) to preserve the metabolites during the collection period. The total urine volume was weighed before samples were stored at $-80^{\circ} \mathrm{C}$ until further analysis.

\section{Blood collection}

During a test day blood samples were taken for the determination of hesperidin, hesperidin $2 S$ and its metabolites. These samples were collected in sodium heparin tubes and immediately centrifuged at $1300 \mathrm{~g}$ for $10 \mathrm{~min}$ at $4^{\circ} \mathrm{C}$. Thirty-six $\mu / / \mathrm{ml} 4 \mathrm{mM}$ hydrochloric acid $(\mathrm{HCl})$ was added to the plasma in support of the LC-MS measurements of the metabolites. All plasma samples were stored at $-80^{\circ} \mathrm{C}$ until further analysis.

\section{LC-MS/MS analysis of metabolites}

LC-MS/MS analysis of the samples revealed the following metabolites: hesperetin3-O-glucuronide $(\mathrm{Hp} 3 \mathrm{G})$, hesperetin-7-O-glucuronide $(\mathrm{Hp} 7 \mathrm{G})$ and hesperetin-7-O-sulphate (Hp7S). The optimum mass spectrometer parameters for the detection of the hesperetin metabolites were optimized, connecting the column inlet directly to a special ionization source called 'Jet Stream'. The source parameters were the following: capillary voltage $-3500 \mathrm{~V}$, charging potential $-500 \mathrm{~V}$, nebulizer pressure 40 (psi), auxiliary gas heated to $275^{\circ} \mathrm{C}$ and introduced at a flow rate of pure nitrogen $9 \mathrm{~L} / \mathrm{min}$. The multiple reaction monitoring (MRM) was the method of choice due to the high selectivity and sensitivity in LC-MS/MS, monitoring 4 transitions for each analysis: hesperetin, $\mathrm{m} / \mathrm{z} 301 \rightarrow 164$; hesperetin glucuronide, $\mathrm{m} / \mathrm{z} 477 \rightarrow 301$; hesperetin diglucuronide, $\mathrm{m} / \mathrm{z} 653 \rightarrow(477) \rightarrow 301$; hesperetin sulfoglucuronide, $\mathrm{m} / \mathrm{z} 557 \rightarrow(477) \rightarrow 301$; hesperetin sulfate, $\mathrm{m} / \mathrm{z} 381 \rightarrow 301$; with a dwell time for each transition of $8 \mathrm{~ms}$. Concentrations of hesperetin diglucuronide and hesperetin sulfoglucuronide metabolites were estimated by synthesized hesperetin 
glucuronide calibration curves $(10,5,1,0.5,0.1 \mu M)$. The cumulative excretion of total hesperetin in $24 \mathrm{~h}$ urine was calculated by adding the concentrations of the 3 different metabolites per test day.

\section{Statistical analyses}

Primary outcome of the study was the bioavailability of micronized hesperidin $2 \mathrm{~S}$, measured by the difference in cumulative excretion of hesperetin in $24 \mathrm{~h}$ urine and by the difference in the 240-min and 600-min area under the plasma concentration-time curves $\left(\mathrm{AUC}_{0-4 \mathrm{~h}}\right.$ and $\mathrm{AUC}_{0-10 \mathrm{~h}}$ ) of plasma total metabolite concentration between micronized hesperidin $2 \mathrm{~S}$ and standard hesperidin. As this is an exploratory study, no formal sample size calculation was made. Based on other studies investigating the bioavailability of polyphenols by measuring the urinary excretion and the plasma concentrations of the metabolites, we estimated that at least 8 participants would be sufficient for evaluation of the primary aim. ${ }^{19,22}$ Baseline characteristics are presented as mean (SD) for numerical variables. The AUCs were calculated using the trapezoidal method. Differences in urinary excretion and plasma AUCs of Hp3G, Hp7G and Hp7S and total hesperetin between intervention groups (micronized hesperidin $2 \mathrm{~S}$ or standard hesperidin) were assessed using linear mixed models with group (micronized hesperidin $2 \mathrm{~S}$ or standard hesperidin) as fixed factor, where an unstructured covariance structure was used for repeated measures. The linear mixed model accounts for the correlation between repeated measures and missing data, where a likelihood approach was used assuming data missing at random. The estimated means and SEM obtained from this model were presented for each group. Two-sided $P$-values $\leq 0.05$ were considered statistically significant. Statistical analysis was performed using IBM SPSS Statistics for Windows (version 21.0, Armonk, NY, USA).

\section{Results}

\section{Study subjects}

Initially, 11 healthy volunteers were enrolled in the study [38\% male; age $27 \pm 8$ yrs; BMI (in $\left.\mathrm{kg} / \mathrm{m}^{2}\right) 22 \pm 2$ ], of which 10 completed the entire study protocol. From the drop-out only baseline characteristics were available.

\section{Urinary metabolites}

The cumulative and relative excretion of hesperetin in $24 \mathrm{~h}$ urine is shown in Table 5.1 and Figure 5.1. The urinary excretion of the individual metabolites $\mathrm{Hp} 3 \mathrm{G}, \mathrm{Hp} 7 \mathrm{G}, \mathrm{Hp} 7 \mathrm{~S}$ and of 
total hesperetin was significantly higher after micronized hesperidin $2 S$ intake compared to standard hesperidin intake $(P=0.044, P=0.024, P=0.018, P=0.032$ respectively). Relative urinary excretion of total hesperetin as a percentage of intake was $3.73 \%$ for the standard hesperidin formulation compared to $5.62 \%$ for the micronized hesperidin $2 \mathrm{~S}$ formulation.

Table 5.1 Ingested dose, plasma AUCs and $24 \mathrm{~h}$ urinary excretion of hesperetin after intake of standard hesperidin and micronized $2 \mathrm{~S} \mathrm{HE}^{\prime}$

\begin{tabular}{lccc}
\hline & Standard HE & Micronized HE 2S & $P$ value \\
\hline Ingested dose $(\mathrm{mg})$ & 222.79 & 222.79 & - \\
$\mathrm{AUC}_{0-4 \mathrm{~h}}(\mu \mathrm{mol} / \mathrm{h} / \mathrm{l})$ & $0.4 \pm 0.1^{2}$ & $1.1 \pm 0.3$ & 0.032 \\
$\mathrm{AUC}_{0-10 \mathrm{~h}}(\mu \mathrm{mol} / \mathrm{h} / \mathrm{l})$ & $4.9 \pm 1.1$ & $7.1 \pm 1.3$ & 0.225 \\
Total urinary excretion $(24 \mathrm{~h})$ & & & \\
$\mu \mathrm{mol}$ & $27.52 \pm 4.53$ & $41.48 \pm 7.35$ & 0.032 \\
$\mathrm{mg}$ & $8.31 \pm 1.37$ & $12.53 \pm 2.22$ & \\
Relative urinary excretion & & & \\
percentage of intake & $3.73 \pm 0.61$ & $5.62 \pm 1.00$ & 0.032 \\
\hline
\end{tabular}

'Differences between standard hesperidin and micronized hesperidin $2 \mathrm{~S}$ tested with linear mixed model. HE, hesperidin. ${ }^{2}$ Estimated mean \pm SEM (all such values)

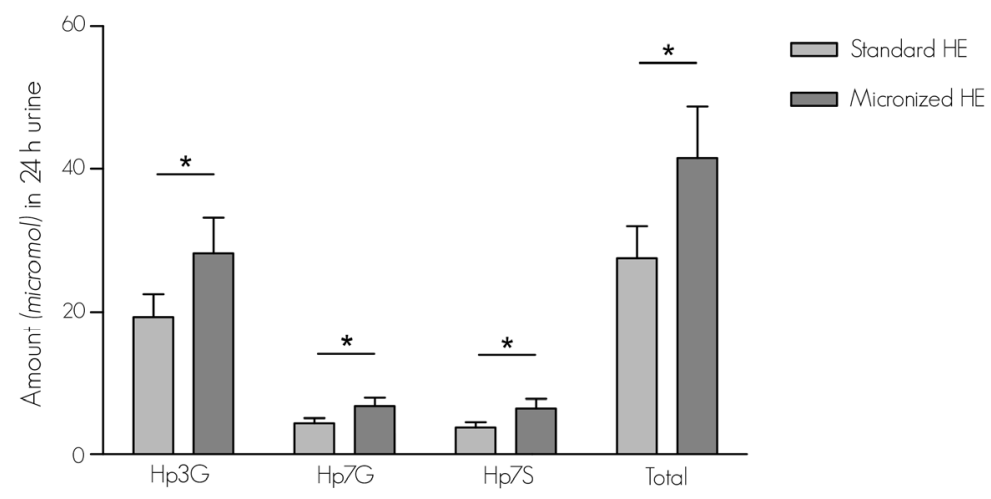

Figure 5.1 The cumulative excretion of $\mathrm{Hp} 3 \mathrm{G}, \mathrm{Hp} 7 \mathrm{G}, \mathrm{Hp} 7 \mathrm{~S}$ and total hesperetin in $24 \mathrm{~h}$ urine after intake of standard hesperidin and micronized hesperidin 2S. Data presented as: mean \pm SEM. Data of standard hesperidin are represented by light gray, data of micronized hesperidin $2 \mathrm{~S}$ by dark gray. Differences between standard hesperidin and micronized hesperidin $2 S$ tested with linear mixed model. * significant $(P \leq 0.050)$ difference between standard hesperidin and micronized hesperidin $2 \mathrm{~S}$.

\section{Plasma metabolites}

The $\mathrm{AUC}_{0-4 h}$ and $\mathrm{AUC}_{0-10 \mathrm{~h}}$ of plasma hesperetin are shown in Table 5.1 and Figure 5.2. The mean and individual time-vs.-plasma concentration curves for hesperetin are shown in Figure 5.3, Figure 5.4A and 5.4B. The $\mathrm{AUC}_{0-4 \mathrm{~h}}$ of plasma hesperetin was significantly higher after micronized hesperidin $2 S$ intake compared to standard hesperidin $(P=0.032)$. However, the 
$\mathrm{AUC}_{0-10 \mathrm{~h}}$ was not significantly different between interventions $(P=0.225)$. Also, there were no significant between-group differences at each measured time point (all $P \geq 0.194$ ).

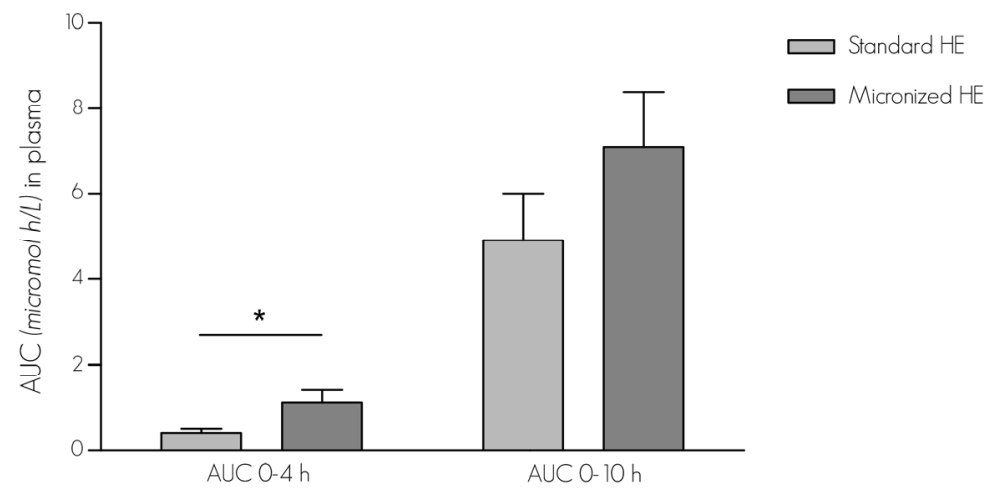

Figure 5.2 The $\mathrm{AUC}_{0-4 \mathrm{~h}}$ and $\mathrm{AUC}_{0-10 \mathrm{~h}}$ of plasma total hesperetin after intake of standard $\mathrm{HE}$ and micronized $2 \mathrm{~S}$ HE. Data presented as: mean \pm SEM. Data of standard HE are represented by light gray, data of micronized $2 \mathrm{~S}$ HE by dark gray. Differences between standard hesperidin and micronized hesperidin $2 S$ tested with linear mixed model. ${ }^{*}$ significant $(P \leq 0.050)$ difference between standard hesperidin and micronized hesperidin $2 \mathrm{~S}$.

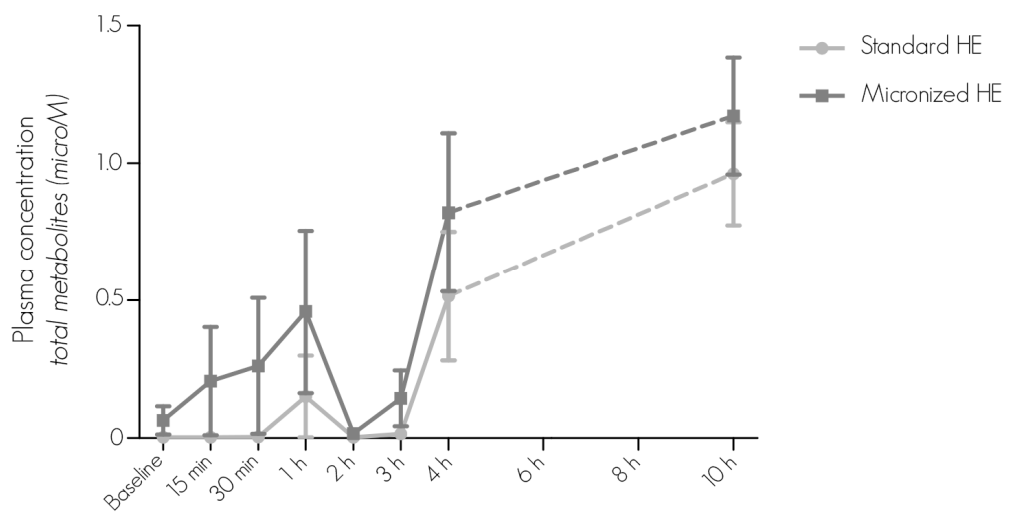

Figure 5.3 Change in plasma concentration of total hesperetin $(\mu M)$ from baseline to $10 \mathrm{~h}$ after intake of standard HE and micronized 2S HE. Data presented as: mean \pm SEM. Data of standard HE are represented by light gray dots, data of micronized $2 S$ HE by dark gray squares. Differences between standard hesperidin and micronized hesperidin $2 S$ tested with linear mixed model. No significant differences in plasma total hesperetin per time point between interventions were observed (all $P \geq 0.194$ ). 


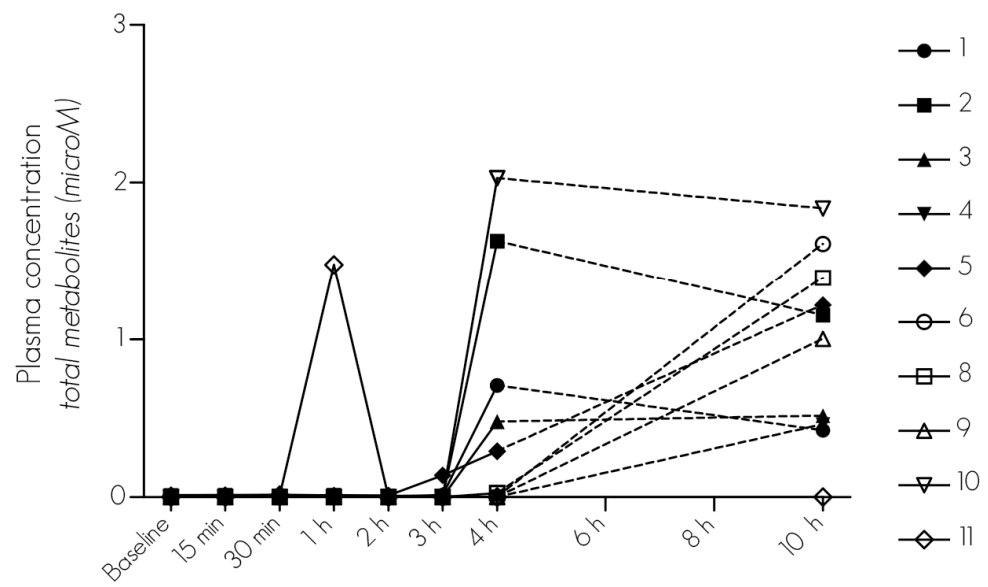

Figure 5.4A Individual plasma concentration curves for total hesperetin $(\mu \mathrm{M})$ from baseline to $10 \mathrm{~h}$ after intake of standard hesperidin. Subject numbers are shown.

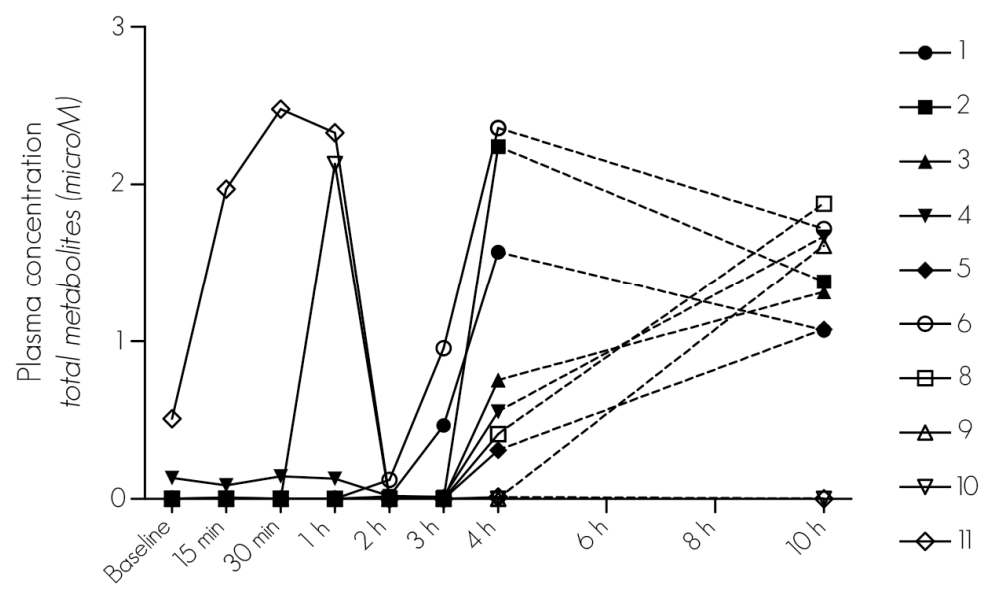

Figure 5.4B Individual plasma concentration curves for total hesperetin $(\mu \mathrm{M})$ from baseline to $10 \mathrm{~h}$ after intake of micronized hesperidin 2S. Subject numbers are shown.

\section{Discussion}

The present study demonstrates that micronized hesperidin $2 S$ is more bioavailable than standard hesperidin, as shown by a significant increase in the cumulative excretion of the individual metabolites $\mathrm{Hp} 3 \mathrm{G}, \mathrm{Hp} 7 \mathrm{G}$ and $\mathrm{Hp} 7 \mathrm{~S}$ and of total hesperetin in $24 \mathrm{~h}$ urine, after 
ingestion of one dose micronized hesperidin 2S, compared to standard hesperidin. Additionally, plasma hesperetin $\mathrm{AUC}_{0-4 \mathrm{~h}}$ and $\mathrm{AUC}_{0-10 \mathrm{~h}}$ increased after intake of micronized hesperidin 2S, compared to standard hesperidin, being significant for the $\mathrm{AUC}_{0-4 \mathrm{~h}}$.

It has been shown that the oral bioavailability of hesperidin is low in humans, due to a combination of factors. ${ }^{7.8}$ Hesperidin is highly water insoluble and it requires colonic microbiota to release and enable the absorption of its aglycone hesperetin. $8,9,11,12$ A way to improve the dissolution process of a product is to reduce the size of the product particles by micronization. ${ }^{23}$ Here, we compared the bioavailability of a micronized hesperidin $2 S$ formulation to that of a non-micronized hesperidin formulation. The micronized $2 \mathrm{~S}$ product showed to be more bioavailable than the non-micronized standard product, by excreting significantly higher amounts of the 3 main hesperidin metabolites in $24 \mathrm{~h}$ urine. Human studies investigating the bioavailability of hesperidin have reported lower relative urinary excretion over 24 h. $7,8,19,24,25$ To assess plasma bioavailability we calculated the $A \mathrm{CC}_{0-10 \mathrm{~h}}$ of the individual metabolites and of total hesperetin. Higher AUCs were observed for the individual metabolites and for total hesperetin after micronized hesperidin $2 S$ intake, compared to placebo, but it did not reach significance. Blood samples were taken at baseline and $15 \mathrm{~min}, 30 \mathrm{~min}, 1 \mathrm{~h}, 2 \mathrm{~h}, 3 \mathrm{~h}, 4 \mathrm{~h}$ and $10 \mathrm{~h}$ after study product intake. We particularly focused on the first $4 \mathrm{~h}$ after study product intake as we expected metabolites to be detected more rapidly in the plasma. We had two reasons to assume this: 1) we expected the micronized hesperidin $2 S$ to be absorbed more rapidly, and 2) we administered the study product while the participant was fasted. Indeed, the individual plasma concentration curves for hesperetin show that in a number of cases detection of metabolites occurs earlier after intake of the micronized hesperidin $2 S$ formulation, compared to the non-micronized hesperidin formulation. However, we have not determined plasma metabolite concentrations between $4 \mathrm{~h}$ and $10 \mathrm{~h}$ after study product intake. Based on literature data, a peak in plasma hesperetin is expected to occur between 5 to $7 \mathrm{~h}$ after study product intake. $7,19,24$ Therefore, the plasma $\mathrm{AUC}_{0-10 \mathrm{~h}}$ is probably not an accurate reflection of the real bioavailability. As hesperetin concentrations are higher at each measured time point after intake of micronized hesperidin $2 S$, it can be assumed that this is also the case between $4 \mathrm{~h}$ and $10 \mathrm{~h}$. Very likely, the plasma $\mathrm{AUC}_{0-10 \mathrm{~h}}$ is an underestimation of the true bioavailability of the micronized product. As we measured plasma metabolites regularly during the first $4 \mathrm{~h}$ after study product intake, we are able to calculate an

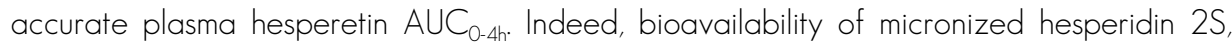
compared to standard hesperidin, showed to be significantly improved already during the first $4 \mathrm{~h}$ after study product intake.

In the current study we observed interindividual variation regarding plasma metabolite excretions. In most subjects the plasma concentration peak lies between 4 and $10 \mathrm{~h}$. The latter indicates that the compound is indeed absorbed from the distal parts of the small intestine or the colon where enzymes, capable of releasing the aglycone hesperetin, are 
present. $^{8,11,12}$ In some subjects, a peak in plasma metabolite concentration was observed within or at $1 \mathrm{~h}$ after study product intake. This might seem to be too short a time for a compound to reach the ileum or colon. However, subjects ingested the study product with water in a fasting state; this might have accelerated transit of the compound through the $G$ l tract. Also, micronizing the compound could have affected the rate of absorption. Interindividual variation in polyphenol bioavailability has also been found in other human intervention studies. ${ }^{7,26-28}$ Most likely, the observed variations are caused by interindividual differences in intestinal microbiota composition and activity. ${ }^{28,29}$ Also, the colonic conversion rate of polyphenols to metabolites can vary greatly between individuals due to differences in transit time and substrate availability. ${ }^{30}$

The present data show that the main circulating metabolites, formed after oral intake of hesperidin, were hesperetin glucuronides and sulphates. This is in line with other trials assessing the bioavailability of hesperidin in humans. ${ }^{3,18}$ Studies investigating the biological effects of these specific metabolites are limited. It has been reported that they beneficially contribute to cardiovascular health. ${ }^{31-33}$ An in vitro study demonstrated that hesperetin glucuronide increased nitric oxide $(\mathrm{NO})$ release from endothelial cells, indicating that it is able to improve vascular dilatation. ${ }^{3}$ A study in hypertensive rats showed that hesperetin glucuronide has blood pressure lowering effects, endothelium-dependent vasodilatory activity and anti-inflammatory properties. ${ }^{31}$ Furthermore, glucuronated and sulphated metabolites of hesperidin showed to reduce the adhesion of monocytes to endothelial cells and to modulate the expression of genes related to inflammation, two important aspects involved in atherogenesis. ${ }^{32}$ Finally, Giménez-Bastida et al. found a reduction of PAl-1, a thrombogenic protein involved in a wide range of CVD as well as in cell migration, by hesperitin and its metabolites. ${ }^{33}$ It should be noted that the above mentioned results regarding the beneficial effects of hesperidin metabolites in cardiovascular health have been obtained from in vitro or animal studies.

A limitation of the present study is the lack of available blood samples between $4 \mathrm{~h}$ and $10 \mathrm{~h}$ after study product intake for measuring hesperidin metabolites. In line with this, we were not able to calculate an accurate $\mathrm{AUC}_{0-10 \mathrm{~h}}$. However, as we regularly obtained blood samples between $\mathrm{O}$ h and $4 \mathrm{~h}$ after study product intake and collected full urine output over $24 \mathrm{~h}$, we could still reliably measure and interpret the bioavailability of hesperidin $2 \mathrm{~S}$ in this study.

In conclusion, the results of this study demonstrate that the bioavailability of micronized hesperidin $2 S$ is higher compared to standard non-micronized hesperidin in healthy individuals. The enhanced bioavailability may positively affect its biological activity. Owing to a great interindividual diversity in intestinal environment, the bioavailability, biological activity and systemic health promoting effects of a polyphenolic compound can be highly variable within the human population. 


\section{References}

1. Bazzano LA, He J, Ogden LG, et al. Fruit and vegetable intake and risk of cardiovascular disease in US adults: the first National Health and Nutrition Examination Survey Epidemiologic Follow-up Study. Am J Clin Nutr. 2002;76(1):93-9.

2. Hooper L, Kroon PA, Rimm EB, et al. Flavonoids, flavonoid-rich foods, and cardiovascular risk: a metaanalysis of randomized controlled trials. Am J Clin Nutr. 2008;88(1):38-50.

3. Takumi $H$, Nakamura $H$, Simizu $T$, et al. Bioavailability of orally administered water-dispersible hesperetin and its effect on peripheral vasodilatation in human subjects: implication of endothelial functions of plasma conjugated metabolites. Food Funct. 2012;3(4):389-98.

4. Rizza S, Muniyappa R, lantorno $M$, et al. Citrus polyphenol hesperidin stimulates production of nitric oxide in endothelial cells while improving endothelial function and reducing inflammatory markers in patients with metabolic syndrome. J Clin Endocrinol Metab. 201 1;96(5):E782-92.

5. Kurowska EM, Spence JD, Jordan J, et al. HDL-cholesterol-raising effect of orange juice in subjects with hypercholesterolemia. Am J Clin Nutr. 2000;72(5): 1095-100.

6. Mulvihill EE, Burke AC, Huff MW. Citrus flavonoids as regulators of lipoprotein metabolism and atherosclerosis. Annu Rev Nutr. 2016 Jul 17;36:275-99.

7. Erlund I, Meririnne E, Alfthan G, Aro A. Plasma kinetics and urinary excretion of the flavanones naringenin and hesperetin in humans after ingestion of orange juice and grapefruit juice. J Nutr. 2001;131(2):235-41.

8. Manach C, Morand C, Gil-lzquierdo A, et al. Bioavailability in humans of the flavanones hesperidin and narirutin after the ingestion of two doses of orange juice. Eur J Clin Nutr. 2003;57(2):235-42.

9. Gil-lzquierdo A, Gil MI, Tomas-Barberan FA, Ferreres F. Influence of industrial processing on orange juice flavanone solubility and transformation to chalcones under gastrointestinal conditions. J Agric Food Chem. 2003:5 1(10):3024-8.

10. Nemeth K, Plumb GW, Berrin JG, et al. Deglycosylation by small intestinal epithelial cell beta-glucosidases is a critical step in the absorption and metabolism of dietary flavonoid glycosides in humans. Eur J Nutr. 2003;42(1):29-42.

11. Amaretti A, Raimondi S, Leonardi A, et al. Hydrolysis of the rutinose-conjugates flavonoids rutin and hesperidin by the gut microbiota and bifidobacteria. Nutrients. 2015;7:2788-800.

12. Matsumoto $H$, Ikoma $Y$, Sugiura $M$, et al. Identification and quantification of the conjugated metabolites derived from orally administered hesperidin in rat plasma. J Agric Food Chem. 2004;52(21): 6653-9.

13. Tomas-Navarro M, Vallejo F, Borrego F, Tomas-Barberan FA. Encapsulation and micronization effectively improve orange beverage flavanone bioavailability in humans. J Agric Food Chem. 2014;62(39):9458-62.

14. Aturki Z, Brandi V, Sinibaldi M. Separation of flavanone-7-O-glycoside diastereomers and analysis in citrus juices by multidimensional liquid chromatography coupled with mass spectrometry. J Agric Food Chem. 2004;52(17):5303-8.

15. Yanez JA, Remsberg CM, Miranda ND, Vega-Villa KR, Andrews PK, Davies NM. Pharmacokinetics of selected chiral flavonoids: hesperetin, naringenin and eriodictyol in rats and their content in fruit juices. Biopharm Drug Dispos. 2008;29(2):63-82.

16. Ariens EJ. Stereochemistry, a basis for sophisticated nonsense in pharmacokinetics and clinical pharmacology. Eur J Clin Pharmacol. 1984;26(6):663-8

17. Brand W, Shao J, Hoek-van den Hil EF, et al. Stereoselective conjugation, transport and bioactivity of s- and R-hesperetin enantiomers in vitro. J Agric Food Chem. 2010;58(10):6 1 19-25.

18. Bredsdorff L, Nielsen IL, Rasmussen SE, et al. Absorption, conjugation and excretion of the flavanones, naringenin and hesperetin from alpha-rhamnosidase-treated orange juice in human subjects. $\mathrm{Br} J \mathrm{Nutr}$. 2010; 103( 1 1): 1602-9.

19. Nielsen IL, Chee WS, Poulsen L, et al. Bioavailability is improved by enzymatic modification of the citrus flavonoid hesperidin in humans: a randomized, double-blind, crossover trial. J Nutr. 2006; 136(2):404-8. 
20. Gonzalez-Barrio R, Trindade LM, Manzanares P, et al. Production of bioavailable flavonoid glucosides in fruit juices and green tea by use of fungal alpha-L-rhamnosidases. J Agric Food Chem. 2004;52(20): 6136-42.

21. Garner RC, Garner JV, Gregory S, et al. Comparison of the absorption of micronized (Daflon $500 \mathrm{mg}$ ) and nonmicronized 14C-diosmin tablets after oral administration to healthy volunteers by accelerator mass spectrometry and liquid scintillation counting. J Pharm Sci. 2002;91(1):32-40.

22. Ross JA, Kasum CM. Dietary flavonoids: bioavailability, metabolic effects, and safety. Annu Rev Nutr. 2002;22:19-34

23. Chaumeil JC. Micronization: a method of improving the bioavailability of poorly soluble drugs. Methods Find Exp Clin Pharmacol. 1998;20(3):21 1-5.

24. Silveira JQ, Cesar TB, Manthey JA, et al. Pharmacokinetics of flavanone glycosides after ingestion of single doses of fresh-squeezed orange juice versus commercially processed orange juice in healthy humans. J Agric Food Chem. 2014;62(52): 12576-84.

25. Vallejo $F$, Larrosa $M$, Escudero $E$, et al. Concentration and solubility of flavanones in orange beverages affect their bioavailability in humans. J Agric Food Chem. 2010;58(10): 6516-24.

26. Cerda B, Tomas-Barberan FA, Espin JC. Metabolism of antioxidant and chemopreventive ellagitannins from strawberries, raspberries, walnuts, and oak-aged wine in humans: identification of biomarkers and individual variability. J Agric Food Chem. 2005;53(2):227-35.

27. Lee MJ, Maliakal P, Chen L, et al. Pharmacokinetics of tea catechins after ingestion of green tea and (-)epigallocatechin-3-gallate by humans: formation of different metabolites and individual variability. Cancer Epidemiol Biomarkers Prev. 2002; 11 (10 Pt 1):1025-32.

28. Rowland IR, Wiseman H, Sanders TA, et al. Interindividual variation in metabolism of soy isoflavones and lignans: influence of habitual diet on equol production by the gut microflora. Nutr Cancer. 2000;36(1):27-32.

29. Bode $L M$, Bunzel $D$, Huch $M$, et al. In vivo and in vitro metabolism of trans-resveratrol by human gut microbiota. Am J Clin Nutr. 2013:97(2):295-309.

30. El Oufir L, Flourie B, Bruley des Varannes S, et al. Relations between transit time, fermentation products, and hydrogen consuming flora in healthy humans. Gut. 1996; 38(6):870-7.

31. Yamamoto $M$, Jokura $H$, Hashizume $K$, et al. Hesperidin metabolite hesperetin-7-O-glucuronide, but not hesperetin-3'-O-glucuronide, exerts hypotensive, vasodilatory, and anti-inflammatory activities. Food Funct. 2013;4(9): 1346-51.

32. Chanet A, Milenkovic D, Claude S, et al. Flavanone metabolites decrease monocyte adhesion to TNFalpha-activated endothelial cells by modulating expression of atherosclerosis-related genes. $\mathrm{Br} \mathrm{J}$ Nutr. 2013; 1 10(4):587-98

33. Gimenez-Bastida JA, Gonzalez-Sarrias A, Vallejo F, et al. Hesperetin and its sulfate and glucuronide metabolites inhibit TNF-alpha induced human aortic endothelial cell migration and decrease plasminogen activator inhibitor-1 (PAl-1) levels. Food Funct. 2016;7:1 18-26. 


$$
\text { (1) }
$$




\section{chapter 6}

Randomized clinical trial on the efficacy of hesperidin 2S on validated cardiovascular biomarkers in healthy overweight individuals

Salden BN, Troost FJ, de Groot E, Stevens YR, Garcés-Rimón M, Possemiers S, Winkens B, Masclee AA

American Journal of Clinical Nutrition 2016; 104: 1523-33 


\section{Abstract}

\section{Background}

Endothelial dysfunction (ED) is involved in the development of atherosclerosis. Hesperidin, a citrus flavonoid with antioxidant and other biological properties, potentially exerts beneficial effects on endothelial function (EF).

\section{Objective}

We investigated the effect of hesperidin $2 \mathrm{~S}$ supplementation on EF in overweight individuals.

\section{Design}

This was a randomized, double-blind, placebo-controlled study in which 68 individuals were randomly assigned to receive hesperidin $2 S(450 \mathrm{mg} / \mathrm{d})$ or a placebo for 6 wk. At baseline and after 6 wk of intervention, flow-mediated dilation (FMD), soluble vascular adhesion molecule-1 (sVCAM-1), soluble intracellular adhesion molecule-1 (sICAM-1), soluble P-selectin (sP-selectin), systolic blood pressure (SBP), and diastolic blood pressure (DBP) were assessed. Acute, reversible ED was induced by intake of a high-fat meal (HFM). A second FMD scan was performed $2 \mathrm{~h}$ postprandially, and adhesion molecules were assessed 2 and $4 \mathrm{~h}$ postprandially. An additional exploratory analysis was performed in subjects with baseline FMD $\geq 3 \%$.

\section{Results}

No significant change in fasting or postprandial FMD was observed after 6 wk of hesperidin intake compared with placebo intake. However, there was a trend for a reduction of sVCAM- 1, sICAM- 1, sP-selectin, SBP, and DBP after 6 wk of hesperidin treatment. In the FMD $\geq 3 \%$ group, hesperidin protected individuals from postprandial ED $(P=0.050)$ and significantly downregulated sVCAM- 1 and sICAM- 1 (all $P \leq 0.030$ ). The results reported in the current article were not adjusted for multiplicity.

\section{Conclusions}

Six weeks of consumption of hesperidin $2 S$ did not improve basal or postprandial FMD in our total study population. There was a tendency toward a reduction of adhesion molecules and a decrease in SBP and DBP. Further exploratory analyses revealed that, in subjects with baseline FMD $\geq 3 \%$, hesperidin $2 \mathrm{~S}$ improved ED after an HFM and reduced adhesion molecules. These results indicate the cardiovascular health benefits of hesperidin $2 S$ in overweight and obese individuals with a relatively healthy endothelium. 


\section{Introduction}

The vascular endothelium is a monolayer of cells between the lumen and vascular smooth muscle cells. The vascular endothelium plays a crucial role in the maintenance of vascular homeostasis by keeping a refined balance between vasodilation and vasoconstriction.' Nitric oxide (NO) is of pivotal importance in the regulation of the arterial tone and, thus, endothelial function (EF). ${ }^{2}$ Chronic exposure to cardiovascular disease (CVD) risk factors [i.e., obesity, ${ }^{3}$ hypertension, ${ }^{4}$ diabetes mellitus $\left.{ }^{5,6}\right]$ and to oxidative stress impairs the endothelium ${ }^{7}$ and may lead to endothelial dysfunction (ED). ED is often manifested by an impaired capacity of the vascular endothelium to dilate as result of insufficient $\mathrm{NO}$ bioavailability. ${ }^{8}$ ED has a key role in the development and progression of atherosclerosis, in which circulating leukocytes are recruited to the vascular endothelium and further migrate into subendothelial spaces, which are mediated by cellular adhesion molecules [i.e., soluble vascular adhesion molecule-1 (sVCAM-1), soluble intracellular adhesion molecule-1 (sICAM-1), soluble E-selectin (sE-selectin), and soluble P-selectin (sP-selectin)]. ${ }^{9}$ Hence, interventions aimed at improving ED may provide an attractive approach in the prevention of atherosclerosis and CVD.

Hesperidin, which is a flavonoid that is abundantly present in the peels of citrus fruit, may have such potential. Hesperidin (hesperetin-7-O-rutinoside), to a large extent, reaches the colon intact where it is subsequently deglycosylated by intestinal microbiota to produce the active aglycone hesperitin. ${ }^{11,12}$ Hesperitin has previously been shown to upregulate endothelial $\mathrm{NO}$-synthase activity and, therefore, has intrinsic potential to protectively affect EF. ${ }^{13}$ However, low solubility limits the bioavailability and resulting biological activity in humans. Furthermore, although natural products typically contain high concentrations of the most-active form of hesperidin (hesperitin-7-O-rutinoside 2S), typical hesperidin extracts mainly contain the less-active $2 R$ enantiomer. ${ }^{14,15}$ To overcome both the limited bioavailability and the typically suboptimal enantiomer composition, we studied a hesperidin $2 S$ extract (Cordiart; BioActor BV) that has a specific enantiomer configuration that is similar to natural hesperidin and was specifically developed for having improved bioavailability profiles.

The primary aim of our study was to investigate the efficacy of 6 wk of supplementation of the specific hesperidin $2 \mathrm{~S}$ formulation on EF as measured with the use of flow-mediated dilation (FMD) in a population of healthy but overweight individuals. We selected overweight individuals because this condition is associated with chronic low-grade inflammation, and in these subjects, mild ED regularly occurs. ${ }^{16}$ Because we recruited from the general population rather than from a selection of a population with proven ED, we induced temporary, reversible ED by providing participants with a high-fat meal (HFM) during test days. ${ }^{17,18}$ This method allowed us to further assess the effect of a single dosage of hesperidin $2 \mathrm{~S}$ on HFM-stressed EF. Our secondary aim was to investigate the 6-wk 
effects of this formulation on circulating adhesion molecules, blood pressure (BP), and metabolic markers. We hypothesized that 6 wk of supplementation with this specific hesperidin $2 \mathrm{~S}$ extract would improve EF, downregulate adhesion molecules, lower systolic blood pressure (SBP) and diastolic blood pressure (DBP), and enhance metabolic control.

\section{Methods}

The study was approved by the Medical Ethics Committee of the Maastricht University Medical Centert and was conducted in full accordance with the principles of the Declaration of Helsinki of 1975 as amended in 2013 and with the Dutch Regulations on Medical Research involving Human Subjects (1998). The study was performed at the Maastricht University Medical Centert from March 2014 to October 2014. All participants gave written informed consent before participation. This trial was registered at clinicaltrials.gov as NCTO2228291.

\section{Subjects}

Healthy volunteers who were aged $18-65$ y and had BMI (in $\mathrm{kg} / \mathrm{m}^{2}$ ) between 25 and 35 were recruited through advertisements in the local media. Key exclusion criteria were as follows: type 2 diabetes mellitus (defined as fasting plasma glucose concentration $\geq 7 \mathrm{mmo} / \mathrm{L}$ ); any medical condition that might have interfered with the study and/or jeopardized the health status of the participant; smoking; abuse of alcohol $1>20$ alcoholic $\mathrm{U} / \mathrm{wk}$ ) and recreational drugs; no consistently stable body weight for $\geq 3 \mathrm{mo}( \pm 3 \mathrm{~kg})$; plans to lose weight or follow an energy-restriction diet during the study period; any medication, vitamin supplements, mineral supplements, or antioxidant supplements during the study or in the $90 \mathrm{~d}$ before start of the study; use of antibiotics in the $90 \mathrm{~d}$ before the start of the study; pregnancy and lactation; history of any side effects toward intake of flavonoids or citrus fruit; and failure to comply with prohibited intake of hesperidin-rich food products and food products that influence EF. For the exploratory subgroup analysis, the following additional exclusion criterion was applied: baseline FMD $<3 \%$. The cutoff of $3 \%$ was based on data from a large meta-analysis on EF. ${ }^{19}$ In this study, it was illustrated that FMD values $<3 \%$ were associated with a Framingham Risk Score of $\sim 40 \%$, thereby making improvements of a shortterm intervention, as in our study, on a chronically impaired endothelium rather unlikely. In addition, the repeatability of low FMD values is decreased, thereby making results of statistical tests that evaluate FMD differences less robust. ${ }^{20,21}$ During the study period, subjects consumed their habitual diets. At the time of inclusion, all subjects were informed about the prohibited hesperidin-rich food products and food products that might potentially influence EF. 


\section{Design and intervention}

This study was designed as a randomized, placebo-controlled, double-blind, parallel-group study (Supplemental Figure S6.1). Each subject underwent 2 test days. Participants were randomly assigned in a double-blind fashion to one of the following 2 intervention arms: hesperidin $2 \mathrm{~S}$ or placebo (cellulose). An independent and blinded person generated the list of random assignment with the use of a computerized procedure. All participants and investigators remained blind to the treatment until all analyses were completed. Participants were requested to abstain from strenuous physical exercise, consumption of alcohol and caffeine, and intake of vitamin $C$ on the day before each test day. ${ }^{22}$ Assessments took place in a quiet, temperature-controlled $\left(20-24^{\circ} \mathrm{C}\right)$ room.

After an overnight fast, subjects arrived at the study site, and anthropometric measurements (height, body weight, and waist-to-hip circumference) were performed. After 30 min of rest in the supine position, subjects underwent BP measurements. Next, we assessed the first ultrasound FMD scan of the right brachial arterial lumen to test EF. After completion of the ultrasound scan, an intravenous catheter was inserted into the antecubital vein of the left arm, and blood samples were collected. Subjects consumed a standardized HFM to induce acute, mild, reversible ED. Synchronously with the HFM, one dosage of hesperidin $2 S$ or placebo was ingested. Exactly $2 \mathrm{~h}$ after meal ingestion, a second FMD scan was performed. Blood sampling was performed at 2 and $4 \mathrm{~h}$ after the meal. At the end of the test day, the intravenous catheter was removed, and participants received the study products for the entire study period. After 6 wk of daily supplementation, test day 2 was organized. Measurements were identical to the measurements performed on test day 1. Figure 6.1 shows the timeline of test days 1 and 2. To assess FMD variability and intrasonographer reproducibility in this specific study, a subset of $10 \%$ of participants underwent repeat FMD scans at study baseline and after 6 wk of supplementation. To assess compliance, participants were asked to save the empty and (partly) full blister packs and to return them at the last visit. Also, in the first week and last week of the study period, participants were asked to fill out a 3-d food record to assess whether they maintained their dietary habits throughout the study period.

The primary aim of our study was to investigate the b-wk effects of the specific hesperidin $2 S$ formulation on fasted and postprandial EF as measured with the use of FMD in healthy but overweight individuals. The secondary aim was to investigate the 6-wk effects of this formulation on circulating adhesion molecules, BP, and metabolic markers. In addition, an exploratory analysis was performed to assess the efficacy of this hesperidin $2 \mathrm{~S}$ extract on FMD with the exclusion of subjects with strongly decreased baseline FMD values $<3 \%$. Because plasma concentrations of adhesion molecules are closely related to FMD values ${ }^{23}$, and factors that influence BP have been proposed to operate via mechanisms that are 
linked to the release of substances from the endothelium ${ }^{24}$, the efficacy of hesperidin $2 S$ regarding these variables was assessed in the FMD $\geq 3 \%$ group.

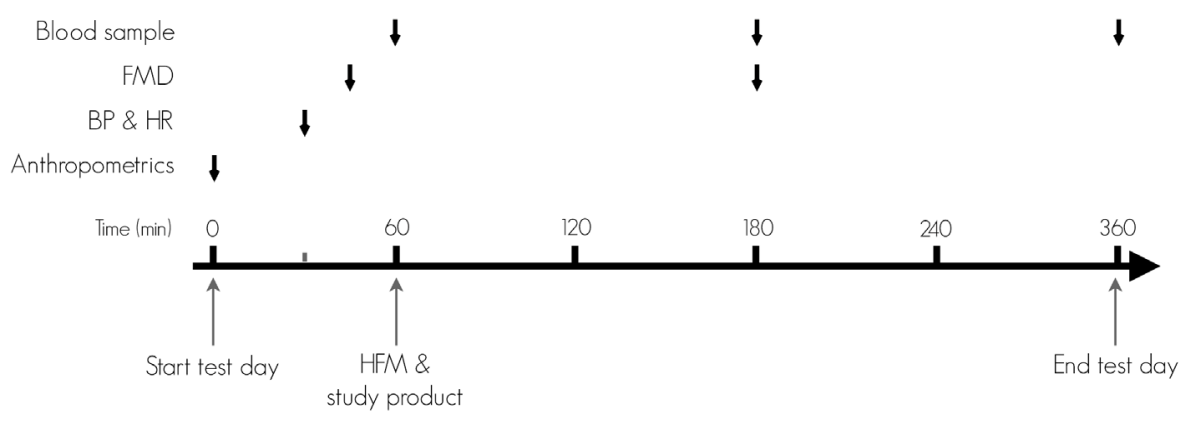

Figure 6.1 Timeline of test days 1 and 2. Blood samples, FMD, BP and HR values, and anthropometric measures were collected at different time points as indicated. BP, blood pressure; FMD, flowmediated dilation; HFM, high-fat meal; HR, heart rate.

\section{Hesperidin and placebo}

Hesperidin 2S (450 mg supplied as 500 mg Cordiart) was extracted from the Citrus sinesis peel, which contained both the $S$ and $R$ enantiomers in the natural 4:1 $S: R$ ratio of hesperidin. Cellulose $(500 \mathrm{mg}$ microcrystalline cellulose; Aminolabs) was administered as the placebo. The study products were formulated into capsules, each of which contained $250 \mathrm{mg}$ of study product or placebo. Subjects were asked to ingest 2 capsules each morning with $200 \mathrm{~mL} \mathrm{H} \mathrm{H}_{2} \bigcirc$ just before the consumption of breakfast for 6 wk.

\section{HFM}

The meal provided during test days was a standardized HFM (2.6 MJ; energy divided as $61 \%$ fat, 33\% carbohydrates, and 6\% protein). The meal consisted of $125 \mathrm{~g}$ whole milk (Friesche Vlag; FrieslandCampina), $15 \mathrm{~g}$ sucrose (Van Gilse), $50 \mathrm{~g}$ whipped cream (Friesche Vlag; FrieslandCampina), and $150 \mathrm{~g}$ vanilla ice cream (Hertog; Unilever Nederland).

\section{FMD}

EF was assessed with the use of B-mode ultrasound brachial artery FMD as described previously. ${ }^{25}$ Two trained and certified sonographers performed the strictly standardized scan protocol. The effect of the intervention on FMD was assessed both without and with an HFM challenge. For quality-control (OC) purposes, on top of FMD measurements that were performed during both study visits, in $\sim 30 \%$ of participants, repeat-fasting @C scans 
were performed at both visits (e.g., 6 scans in total). In a given participant, all FMD measurements were done by the same sonographer. Thus, the sonographer performed both FMD measurements on test days 1 and 2 and, if applicable, the QC FMD measurements of the participant. B-mode ultrasound scans of the diameter of the right brachial artery lumen were obtained with the sue of a Sonix Touch ultrasound machine (Ultrasonix) that was equipped with a 7.5- $\mathrm{MHz}$ linear array probe as well as a study-specific, ultrasoundinstrument scan protocol and settings as applied by the imaging core laboratory (Imagelabonline \& Cardiovascular). A special probe-holder arm rest was used to optimize the stability and standardization of the position of the ultrasound probe. A BP cuff was placed around the forearm $\sim 1 \mathrm{~cm}$ below the antecubital fossa. At rest, measurements were obtained during 1 min after which the BP cuff was inflated to $250 \mathrm{~mm} \mathrm{Hg}$ to occlude the brachial artery. After 5 min of forearm ischemia, the cuff was released, and brachial diameter measurements were continuously recorded for 3 min after cuff release. Image acquisition was electrocardiogram gated on the $\mathrm{R}$ wave, and the ultrasound images were saved in a Digital Imaging and Communications in Medicine clip. For $\bigcirc$ C purposes and to allow for repeat scans in the case of a methodologic failure, all study scans, together with their completed web-based scan forms, were transferred securely to the core laboratory after finishing a scan. For all scans, the imaging technicians of the core laboratory instantly provided an evaluation and feedback of the sonographer scan quality. To be accepted, a precuff occlusion difference diameter of the brachial artery between follow-up study scans had to be visualized in the $\leq 0.2-\mathrm{mm}$ range.

\section{Image analysis}

Accepted scans were securely stored locally on the hard-disk drive of the ultrasound instrument and automatically backed-up on the Imagelabonline \& Cardiovascular core laboratory server. Available scans were batched in a per-subject fashion. The imageanalysis technician was dedicated to analyze all scans of a subject and was blind to the clinical information, date of the scan, and intervention. Validated brachial FMD software (Brachial Analyzer version 6.2.3; Medical Imaging Applications) was used. In summary, the technician selected a region of interest in the longitudinal image of the brachial artery walls and lumen. The Brachial Analyzer software performed an automated tracing of the lumenwall interfaces, ${ }^{26}$ quantifying the brachial artery lumen diameter before cuff occlusion and after cuff release. Mean preocclusion diameters and the maximum after-cuff-release diameters were used to calculate ${ }^{22}$ the absolute (peak diameter minus baseline diameter) and FMD [lpeak diameter - baseline diameter)/baseline diameter] ${ }^{*} 100 \%$. After the first batched series of 30 subjects, the remaining scans of subjects were analyzed. 


\section{Adhesion molecules}

During a test day, blood samples were collected in K2EDTA-coated evacuated tubes for the measurement of adhesion molecules when subjects were fasted and at 2 and $4 \mathrm{~h}$ after HFM intake, respectively. These samples were centrifuged at $1100 \times \mathrm{g}$ for $15 \mathrm{~min}$ at $4^{\circ} \mathrm{C}$ to obtain the plasma, which was divided into aliquots and kept frozen at $-80^{\circ} \mathrm{C}$ until further analysis. Circulating sVCAM-1, sICAM-1, and sE-selectin in plasma were determined by commercially available ELISA kits and standards, according to the manufacturer's protocol (R\&D System Europe Ltd.). Circulating plasma sP-selectin was measured by a quantitative sandwich immunoassay technique (R\&D System Europe Ltd.).

\section{Blood pressure measurements}

BP was monitored with the use of a semicontinuous BP-monitoring device (Omron) on the upper left arm. At each occasion, in total, 4 BP measurements were performed. The first measurement was discarded, and the remaining 3 measurements were averaged. Measurements took place at baseline and at the end of the study period. During a test day, BP was measured before HFM consumption.

\section{Blood lipids, glucose and insulin analysis}

During a test day, blood samples were taken for the assessment of metabolic variables when subjects had fasted. Serum concentrations of glucose, total cholesterol, LDL cholesterol, HDL cholesterol, and triglycerides were measured with the use of spectrophotometry (Cobas 6000 analyzer series; Roche Diagnostics). Plasma insulin was determined with the use of a luminescence-enhanced immune-enzymatic assay IImmulite 2000 immunoassay system; Siemens Healthcare). Insulin sensitivity was estimated with the use of a quantitative insulin-sensitivity check index ${ }^{27}$ as: $1 /(\log$ insulin $\mathrm{O} h+\log$ glucose $\mathrm{O} h$ ).

\section{Statistical analyses}

The primary outcome of the study was the effect of 6 wk of hesperidin $2 S$ supplementation on FMD compared with that of a placebo. As our secondary outcome, we investigated the effect of 6 wk of hesperidin $2 S$ supplementation compared with that of placebo on adhesion molecules, BP, and metabolic variables. In addition, the postprandial effect of hesperidin $2 \mathrm{~S}$ on EF was investigated. The sample size was determined for the primary outcome of the study with a significance-level $\alpha=0.05$ and a power of $80 \%$. On the basis of previous work, ${ }^{28}$ we calculated that a sample size of 62 subjects would be required to detect a mean \pm SD difference in FMD of $1.45 \% \pm 2.0 \%$. Baseline characteristics are 
presented as means \pm SDs for numerical variables and numbers (percentages) for categorical variables. Differences in FMD, BP, and blood variables between intervention groups (hesperidin $2 \mathrm{~S}$ or placebo) were assessed with the use of linear mixed models with the group (placebo and hesperidin 2S), time 10 and 6 wk for the 6 -wk hesperidin effect; 0 , 2 , and $4 \mathrm{~h}$ for the postprandial hesperidin effect), and group $\mathrm{x}$ time as fixed factors, whereby a marginal model with an unstructured covariance structure was used for repeated measures (no random effects). The linear mixed model accounts for the correlation between repeated measures and missing data for which a likelihood approach was used with the assumption of data missing at random. The estimated means \pm SEMs that were obtained from this model were presented for each group and each time point. A 2-sided $P \leq 0.05$ was considered statistically significant. The statistical analysis was performed with the use of IBM SPSS Statistics for Windows software (version 21.0; IBM).

\section{Results}

\section{Study subjects}

Initially, 68 healthy volunteers were enrolled in the study of whom 63 subjects completed the entire study protocol. Three participants were included and randomly assigned to a treatment but never started participation because of personal reasons. From these participants, only baseline characteristics were available. Another participant terminated the study prematurely because of an occurrence of a skin rash, which disappeared after stopping the study product. We excluded 1 participant during the study period because of overt noncompliance. The FMD $\geq 3 \%$ group entailed 48 subjects with baseline FMD $\geq 3 \%$. Baseline characteristics in the total study population and in the FMD $\geq 3 \%$ group are presented in Table 6.1. Dietary habits of the participants were maintained throughout the entire study period.

Table 6.1 Baseline characteristics in the total study population and FMD $\geq 3 \%$ group.'

\begin{tabular}{lcccccc}
\hline & $\begin{array}{c}\text { Total population } \\
(n=68)\end{array}$ & $\begin{array}{c}\text { Placebo } \\
(n=34)\end{array}$ & $\begin{array}{c}\text { Hesperidin } \\
(n=34)\end{array}$ & $\begin{array}{c}F M D \geq 3 \% \text { group } \\
(n=48)\end{array}$ & $\begin{array}{c}\text { Placebo } \\
(n=24)\end{array}$ & $\begin{array}{c}\text { Hesperidin } \\
(n=24)\end{array}$ \\
\hline Age, y & $53 \pm 14^{2}$ & $53 \pm 14$ & $54 \pm 15$ & $50 \pm 15$ & $50 \pm 14$ & $50 \pm 16$ \\
Sex, M/F, $n$ & $29 / 39$ & $12 / 22$ & $17 / 17$ & $17 / 31$ & $5 / 19$ & $12 / 12$ \\
WHR & $0.94 \pm 0.06$ & $0.95 \pm 0.06$ & $0.94 \pm 0.06$ & $0.94 \pm 0.06$ & $0.94 \pm 0.06$ & $0.95 \pm 0.05$ \\
$\mathrm{BMI}, \mathrm{kg} / \mathrm{m}^{2}$ & $29.0 \pm 2.6$ & $29.7 \pm 2.8$ & $28.2 \pm 2.2$ & $29.1 \pm 2.8$ & $29.8 \pm 3.0$ & $28.5 \pm 2.4$ \\
\hline
\end{tabular}

${ }^{1} F M D$, flow-mediated dilation; WHR, waist-to-hip-ratio. ${ }^{2}$ Mean \pm SD (all such values) 


\section{Effect of HFM}

As shown in Table 6.2, a nonsignificant decrease in FMD was observed after the ingestion of an HFM in both the placebo and the hesperidin group. The size of the decrease in postprandial FMD did not significantly differ between the 2 intervention groups $(P=0.670)$.

\section{Effects of HFM in subjects with FMD $\geq 3 \%$}

In the FMD $\geq 3 \%$ group, the ingestion of an HFM reduced FMD in both the placebo and hesperidin groups. The size of the decrease in postprandial FMD did not significantly differ between the 2 intervention groups $(P=0.618$ ) (Table 6.2)

Table 6.2 Postprandial FMD after a single dosage in the total study population (placebo: $n=32$; hesperidin: $n=33$ ) and in the FMD $\geq 3 \%$ group (placebo: $n=24$; hesperidin: $n=24$ ) at baseline (test day 1 ). ${ }^{1}$

\begin{tabular}{lccccc}
\hline & \multicolumn{2}{c}{ Placebo } & \multicolumn{2}{c}{ Hesperidin } & $P$ value \\
\cline { 2 - 4 } & Baseline fasting & 2h after HFM & Baseline fasting & 2h after HFM & \\
\hline FMD (\%) & $5.57 \pm 0.51$ & $5.08 \pm 0.53$ & $4.50 \pm 0.51$ & $4.23 \pm 0.51$ & 0.670 \\
Total study population & $7.03 \pm 0.48$ & $6.37 \pm 0.55$ & $5.37 \pm 0.48$ & $5.04 \pm 0.54$ & 0.618 \\
\hline $3 \%$ group &
\end{tabular}

${ }^{1}$ All values are estimated means \pm SEMs. Differences between placebo and hesperidin were tested with the use of a linear mixed model with correction for baseline values. There was no significant FMD decrease over time within intervention groups. FMD, flow-mediated dilation; HFM, high-fat meal.

\section{Six-week effect: fasted}

Fasted FMD did not change after 6 wk of hesperidin $2 S$ supplementation compared with placebo intake $(P=0.881$ ) (Table 6.3).

\section{Six-week effect: fasted, in subjects with FMD $\geq 3 \%$}

No significant difference was observed in fasted brachial FMD between both interventions after 6 wk of supplementation $(P=0.872$ ) (Table 6.3).

Table 6.3 Fasted FMD at baseline and after 6 wk of supplementation in total study population (placebo: $n=32$; hesperidin: $n=33$ ) and in the FMD $\geq 3 \%$ group (placebo: $n=24$; hesperidin: $n=24$ ).

\begin{tabular}{lccccc}
\hline & \multicolumn{2}{c}{ Placebo } & \multicolumn{2}{c}{ Hesperidin } & $P$ value \\
\cline { 2 - 4 } & Baseline & End & Baseline & End & \\
\hline FMD $(\%)$ & & & & & \\
Total study population & $5.57 \pm 0.51$ & $5.43 \pm 0.47$ & $4.50 \pm 0.51$ & $4.29 \pm 0.47$ & 0.881 \\
FMD $\geq 3 \%$ group & $7.03 \pm 0.48$ & $6.47 \pm 0.49$ & $5.37 \pm 0.48$ & $4.89 \pm 0.50$ & 0.872 \\
\hline
\end{tabular}

${ }^{1}$ All values are estimated means \pm SEMs. Differences between placebo and hesperidin were tested with the use of a linear mixed model with correction for baseline values.. FMD, flow-mediated dilation. 
Six-week effect: postprandial

Postprandial FMD at 6 wk also did not significantly differ compared with the placebo $(P=$ 0.207) (Table 6.4).

\section{Six-week effect: postprandial, in subjects with FMD $\geq 3 \%$}

After inducing ED by an HFM, hesperidin $2 S$ significantly protected postprandial FMD from impairment compared with the effect of the placebo $(P=0.050$ ) (Table 6.4). However, this effect was nonsignificant after any correction for multiple testing (e.g., Bonferroni correction for 8 tests performed as shown in Table 6.4).

\section{Variability assessment}

In total, 34 OC FMD scans were available of 19 study participants. The overall (mean) absolute difference between study FMD measurements and OC FMD measurements of complete FMD measurement sets was $0.87 \%$.

Table 6.4 Postprandial FMD after 6 wk of supplementation (test day 2 ) in the total study population (placebo: $n=32$; hesperidin: $n=33$ ) and in the FMD $\geq 3 \%$ group (placebo: $n=24$; hesperidin: $n=24)^{1}$

\begin{tabular}{lccccc}
\hline & \multicolumn{2}{c}{ Placebo } & \multicolumn{2}{c}{ Hesperidin } & $P$ value \\
\cline { 2 - 4 } & Baseline fasting & 2h after HFM & Baseline fasting & 2h after HFM & \\
\hline FMD \% & & & & & \\
Total study population & $5.57 \pm 0.51$ & $5.08 \pm 0.53$ & $4.21 \pm 0.48$ & $4.38 \pm 0.51$ & 0.207 \\
FMD $\geq 3 \%$ group & $6.47 \pm 0.49$ & $5.81 \pm 0.56$ & $4.85 \pm 0.51$ & $5.28 \pm 0.59$ & 0.050 \\
\hline
\end{tabular}

${ }^{1}$ All values are estimated means \pm SEMs. Differences between placebo and hesperidin were tested with the use of a linear mixed model with correction for baseline values.. FMD, flow-mediated dilation; HFM, high-fat meal.

\section{Adhesion molecules}

\section{Six-week effect: fasted}

Changes in basal circulating adhesion molecules after 6 wk of intervention are shown in Table 6.5. A borderline significant downregulation of the adhesion molecules sVCAM- 1 $(P=0.052)$ and sICAM- $1(P=0.056)$ was observed after 6 wk of supplementation with hesperidin $2 \mathrm{~S}$ compared with the placebo. Furthermore, a downregulation of sP-selectin was seen in the hesperidin group, but this effect was NS ( $P=0.086)$. No significant differences in sE-selectin concentrations were observed between the 2 treatments $(P=0.246)$. 
Six-week effect: fasted, in subjects with FMD $\geq 3 \%$

Although a significant downregulation of basal circulating sVCAM-1 $(P=0.030)$ and sICAM- $1(P=0.017)$ was observed after 6 wk of hesperidin $2 S$ supplementation compared with placebo intake, these effects were no longer significant after any multiple-testing correction (e.g., Bonferroni correction with 5 tests performed as shown in Table 6.5). Concentrations of sE-selectin and sP-selectin were not significantly different between the 2 intervention groups $(P=0.211$ and $P=0.936$, respectively)

Table 6.5 Basal circulating adhesion molecules at baseline and after 6 wk of supplementation in the total study population (placebo: $n=32$; hesperidin: $n=33$ ) and in the FMD $\geq 3 \%$ group (placebo: $n=24$; hesperidin: $n=24)^{1}$

\begin{tabular}{|c|c|c|c|c|c|}
\hline & \multicolumn{2}{|c|}{ Placebo } & \multicolumn{2}{|c|}{ Hesperidin } & \multirow[t]{2}{*}{$P$ value } \\
\hline & Baseline & End & Baseline & End & \\
\hline \multicolumn{6}{|c|}{ Total study population, $\mathrm{ng} / \mathrm{mL}$} \\
\hline sVCAM- 1 & $214 \pm 10$ & $215 \pm 10$ & $210 \pm 10$ & $190 \pm 10$ & 0.052 \\
\hline sICAM- 1 & $107 \pm 5$ & $107 \pm 5$ & $110 \pm 5$ & $100 \pm 5$ & 0.056 \\
\hline sE-selectin & $12 \pm 1$ & $11 \pm 2$ & $11 \pm 1$ & $12 \pm 2$ & 0.246 \\
\hline sP-selectin & $78 \pm 5$ & $83 \pm 5$ & $94 \pm 5$ & $83 \pm 5$ & 0.086 \\
\hline \multicolumn{6}{|c|}{$\mathrm{FMD} \geq 3 \%$ group, $\mathrm{ng} / \mathrm{mL}$} \\
\hline sVCAM-1 & $210 \pm 11$ & $215 \pm 12$ & $208 \pm 11$ & $184 \pm 12$ & 0.030 \\
\hline sICAM- 1 & $104 \pm 5$ & $107 \pm 5$ & $114 \pm 5$ & $103 \pm 5$ & 0.017 \\
\hline sE-selectin & $12 \pm 2$ & $12 \pm 2$ & $11 \pm 2$ & $11 \pm 2$ & 0.936 \\
\hline sP-selectin & $76 \pm 5$ & $84 \pm 6$ & $94 \pm 5$ & $87 \pm 6$ & 0.211 \\
\hline
\end{tabular}

${ }^{1}$ All values are estimated means \pm SEMs. Differences between placebo and hesperidin were tested with the use of a linear mixed model with correction for baseline values.. FMD, flow-mediated dilation; sE-selectin, soluble Eselectin; sICAM-1, soluble intercellular adhesion molecule-1; sP-selectin, soluble P-selectin; sVCAM-1, soluble vascular cell adhesion molecule- 1 .

\section{Six-week effect: postprandial}

The effect on postprandial adhesion molecule concentrations at 6 wk was not significantly different between both interventions (all $P \geq 0.154$ ) (Table 6.6).

Six-week effect: postprandial, in subjects with FMD $\geq 3 \%$

In the subgroup analysis, no significant differences in postprandial adhesion molecule concentrations were observed between both interventions (all $P \geq 0.123$ ) (Table 6.6). 
Table 6.6 Postprandial circulating adhesion molecules after 6 wk of supplementation (test day 2) in the total study population (placebo: $n=32$; hesperidin: $n=33$ ) and in the FMD $\geq 3 \%$ group (placebo: $n=24$; hesperidin: $n=24)^{1}$

\begin{tabular}{|c|c|c|c|c|c|c|c|c|}
\hline & \multicolumn{3}{|c|}{ Placebo } & \multicolumn{3}{|c|}{ Hesperidin } & \multirow[t]{2}{*}{$P_{1}$} & \multirow[t]{2}{*}{$P_{2}$} \\
\hline & $\begin{array}{c}\text { Baseline } \\
\text { fasting }\end{array}$ & $\begin{array}{c}2 \mathrm{~h} \text { after } \\
\text { HFM }\end{array}$ & $\begin{array}{c}\text { 4h after } \\
\text { HFM }\end{array}$ & $\begin{array}{l}\text { Baseline } \\
\text { fasting }\end{array}$ & $\begin{array}{c}2 \mathrm{~h} \text { after } \\
\text { HFM }\end{array}$ & $\begin{array}{c}\text { 4h after } \\
\text { HFM }\end{array}$ & & \\
\hline \multicolumn{9}{|c|}{ Total study population, ng/mL } \\
\hline sVCAM- $1^{2}$ & $215 \pm 10$ & $208 \pm 9$ & $200 \pm 10$ & $190 \pm 10$ & $185 \pm 9$ & $180 \pm 10$ & 0.686 & 0.462 \\
\hline sICAM- $1^{2}$ & $107 \pm 5$ & $105 \pm 5$ & $102 \pm 5$ & $100 \pm 5$ & $98 \pm 5$ & $96 \pm 5$ & 0.857 & 0.976 \\
\hline sE-selectin ${ }^{2}$ & $11 \pm 2$ & $9 \pm 2$ & $10 \pm 2$ & $12 \pm 2$ & $11 \pm 2$ & $11 \pm 2$ & 0.154 & 0.358 \\
\hline sP-selectin & $83 \pm 5$ & - & $94 \pm 5$ & $83 \pm 5$ & - & $89 \pm 5$ & - & 0.479 \\
\hline \multicolumn{9}{|c|}{$\mathrm{FMD} \geq 3 \%$ group, $\mathrm{ng} / \mathrm{mL}$} \\
\hline sVCAM- $7^{2}$ & $216 \pm 12$ & $211 \pm 10$ & $203 \pm 11$ & $184 \pm 12$ & $179 \pm 10$ & $171 \pm 11$ & 0.999 & 0.988 \\
\hline sICAM- $1^{2}$ & $107 \pm 5$ & $106 \pm 6$ & $103 \pm 5$ & $103 \pm 5$ & $101 \pm 6$ & $97 \pm 5$ & 0.841 & 0.756 \\
\hline sE-selectin ${ }^{2}$ & $12 \pm 2$ & $10 \pm 2$ & $10 \pm 2$ & $11 \pm 2$ & $11 \pm 2$ & $11 \pm 2$ & 0.182 & 0.123 \\
\hline sP-selectin & $84 \pm 6$ & - & $96 \pm 6$ & $87 \pm 6$ & - & $95 \pm 6$ & - & 0.555 \\
\hline
\end{tabular}

${ }^{1}$ All values are estimated means \pm SEMs. Differences between placebo and hesperidin were tested with the use of a linear mixed model with correction for baseline values.. $P 1$ represents $P$ values for the analysis of baseline compared with $2 \mathrm{~h}$ after the HFM between placebo and hesperidin. $P 2$ represents $P$ values for the analysis of baseline compared 4 h after the HFM between placebo and hesperidin. FMD, flow-mediated dilation; HFM, highfat meal; sE-selectin, soluble E-selectin; sICAM-1, soluble intercellular adhesion molecule-1; sP-selectin, soluble Pselectin; sVCAM-1, soluble vascular cell adhesion molecule- 1. ${ }^{2}$ Time by treatment interaction, $P>0.050$.

\section{Acute effect}

After intake of the HFM with one dosage of hesperidin 2S, there was a trend (P between 0.05 and 0.10 ) that was observed for a downregulation of the adhesion molecule sICAM- 1 at both $2 \mathrm{~h}(P=0.088$ ) (Table 6.7) and $4 \mathrm{~h}(P=0.092$ ) (Table 6.7). No significant postprandial differences were observed regarding sVCAM-1, sE-selectin, and sP-selectin between both study groups (all $P \geq 0$. 193) (Table 6.7).

\section{Acute effect in subjects with FMD $\geq 3 \%$}

At baseline, after intake of an HFM with one dosage of hesperidin 2S (Table 6.7), we observed a significant downregulation of sICAM- 1 after both $2 \mathrm{~h}(P=0.035)$ and $4 \mathrm{~h}(P=$ $0.050)$ and a trend for a downregulation of sE-selectin after $4 \mathrm{~h}(P=0.062)$. Again, after any multiple testing correction (e.g., Bonferroni correction with 8 tests performed as shown in Table 6.7), these effects were no longer significant. No significant postprandial differences were observed regarding sVCAM- 1 and sP-selectin between both study groups lall $P \geq$ 0.1111 . 
Table 6.7 Postprandial adhesion molecules after a single dosage in the total study population (placebo: $n=32$; hesperidin: $n=33$ ) and in the FMD $\geq 3 \%$ group (placebo: $n=24$; hesperidin: $n=24$ ) at baseline (test day 1).

\begin{tabular}{|c|c|c|c|c|c|c|c|c|}
\hline & \multicolumn{3}{|c|}{ Placebo } & \multicolumn{3}{|c|}{ Hesperidin } & \multirow[t]{2}{*}{$P_{1}$} & \multirow[t]{2}{*}{$P_{2}$} \\
\hline & $\begin{array}{c}\text { Baseline } \\
\text { fasting }\end{array}$ & $\begin{array}{c}2 \mathrm{~h} \text { after } \\
\text { HFM }\end{array}$ & $\begin{array}{c}\text { 4h after } \\
\text { HFM }\end{array}$ & $\begin{array}{c}\text { Baseline } \\
\text { fasting }\end{array}$ & $\begin{array}{c}\text { 2h after } \\
\text { HFM }\end{array}$ & $\begin{array}{c}\text { 4h after } \\
\text { HFM }\end{array}$ & & \\
\hline \multicolumn{9}{|c|}{ Total study population, ng/mL } \\
\hline sVCAM- $1^{2}$ & $214 \pm 10$ & $213 \pm 9$ & $206 \pm 10$ & $210 \pm 10$ & $207 \pm 9$ & $209 \pm 10$ & 0.818 & 0.489 \\
\hline sICAM- $1^{2}$ & $107 \pm 5$ & $105 \pm 4$ & $109 \pm 5$ & $110 \pm 5$ & $100 \pm 4$ & $104 \pm 5$ & 0.088 & 0.092 \\
\hline sE-selectin ${ }^{2}$ & $12 \pm 1$ & $12 \pm 2$ & $12 \pm 2$ & $11 \pm 1$ & $10 \pm 2$ & $11 \pm 2$ & 0.356 & 0.998 \\
\hline sP-selectin & $78 \pm 5$ & - & $83 \pm 4$ & $94 \pm 5$ & - & $90 \pm 4$ & - & 0.193 \\
\hline \multicolumn{9}{|c|}{$\mathrm{FMD} \geq 3 \%$ group, $\mathrm{ng} / \mathrm{mL}$} \\
\hline sVCAM- $1^{2}$ & $210 \pm 11$ & $215 \pm 10$ & $206 \pm 11$ & $208 \pm 11$ & $199 \pm 10$ & $202 \pm 11$ & 0.139 & 0.863 \\
\hline sICAM- $1^{2}$ & $104 \pm 5$ & $103 \pm 5$ & $108 \pm 6$ & $114 \pm 5$ & $102 \pm 5$ & $107 \pm 6$ & 0.035 & 0.050 \\
\hline sE-selectin ${ }^{2}$ & $12 \pm 2$ & $12 \pm 2$ & $12 \pm 2$ & $11 \pm 2$ & $10 \pm 2$ & $10 \pm 2$ & 0.111 & 0.062 \\
\hline sP-selectin & $76 \pm 5$ & - & $83 \pm 5$ & $94 \pm 5$ & - & $91 \pm 5$ & - & 0.263 \\
\hline
\end{tabular}

${ }^{1}$ All values are estimated means \pm SEMs. Differences between placebo and hesperidin were tested with the use of a linear mixed model with correction for baseline values.. $P 1$ represents $P$ values for the analysis of baseline compared with $2 \mathrm{~h}$ after the HFM between placebo and hesperidin. $P 2$ represents $P$ values for the analysis of baseline compared th after the HFM between placebo and hesperidin. FMD, flow-mediated dilation; HFM, highfat meal; sE-selectin, soluble E-selectin; sICAM-1, soluble intercellular adhesion molecule-1; sP-selectin, soluble P-selectin; sVCAM- 1, soluble vascular cell adhesion molecule- 1. ${ }^{2}$ Time by treatment interaction, $P>0.050$.

\section{Blood pressure}

\section{Six-week effect: fasted}

Results regarding the effect of hesperidin $2 \mathrm{~S}$ administration on BP are given in Table 6.8. In the hesperidin 2S group, we observed a mean reduction of $5 \mathrm{~mm} \mathrm{Hg}$ in SBP after 6 wk of intake, whereas in the placebo group, we observed a mean reduction of $2 \mathrm{~mm} \mathrm{Hg}$ in SBP. DBP was reduced by $2 \mathrm{~mm} \mathrm{Hg}$ after hesperidin $2 S$ supplementation, whereas DBP increased by $1 \mathrm{~mm} \mathrm{Hg}$ after placebo supplementation. A trend ( $P$ between 0.05 and 0.10 ) toward a lower SBP $(P=0.095)$ and DBP $(P=0.095)$ was observed after 6 wk of hesperidin $2 \mathrm{~S}$ supplementation compared with placebo intake.

\section{Six-week effect: fasted, in subjects with FMD $\geq 3 \%$}

After 6 wk of hesperidin $2 S$ intake, a trend (P between 0.05 and 0.10 ) toward a reduction in both SBP $(P=0.051)$ and DBP $(P=0.069)$ in the FMD $\geq 3 \%$ group was observed compared with in the placebo group. 
Table 6.8 BP and HR at baseline and after 6 wk of supplementation in the total study population (placebo: $n=32$; hesperidin: $n=33$ ) and in the FMD $\geq 3 \%$ group (placebo: $n=24$; hesperidin: $n=24$ ). ${ }^{1}$

\begin{tabular}{lccccc}
\hline & \multicolumn{2}{c}{ Placebo } & \multicolumn{2}{c}{ Hesperidin } & P value \\
\cline { 2 - 4 } & Baseline & End & Baseline & End & \\
\hline Total study population & $131 \pm 3$ & $129 \pm 2$ & $135 \pm 2$ & $130 \pm 2$ & 0.095 \\
Systolic BP, mmHg & $80 \pm 2$ & $81 \pm 2$ & $83 \pm 1$ & $81 \pm 2$ & 0.095 \\
Diastolic BP, mmHg & $62 \pm 1$ & $61 \pm 1$ & $61 \pm 1$ & $62 \pm 1$ & 0.220 \\
HR, beats/min & $128 \pm 2$ & $127 \pm 2$ & $135 \pm 2$ & $129 \pm 2$ & 0.051 \\
FMD $\geq 3 \%$ group & $79 \pm 2$ & $79 \pm 2$ & $84 \pm 2$ & $81 \pm 2$ & 0.069 \\
Systolic BP, mmHg & $62 \pm 2$ & $61 \pm 2$ & $62 \pm 2$ & $63 \pm 2$ & 0.256 \\
Diastolic BP, mmHg & & & & \\
HR, beats/min & & & & & \\
\hline
\end{tabular}

${ }^{1}$ All values are estimated means \pm SEMs. Differences between placebo and hesperidin were tested with the use of a linear mixed model with correction for baseline values.. BP, blood pressure; FMD, flow-mediated dilation; HR, heart rate.

\section{Blood lipids, glucose and insulin (six-week effect: fasted)}

Baseline total cholesterol, LDL cholesterol, HDL cholesterol, triglycerides, glucose, and insulin were all within normal blood value ranges. These variables were not significantly altered by 6 wk of supplementation with hesperidin $2 S$ compared with the placebo lall $P \geq$ 0.097 ) (Table 6.9). Also, no significant change in insulin sensitivity was observed between intervention groups $(P=0.225)$ (Table 6.9).

Table 6.9 Blood lipids, glucose, insulin, and QUICKI at baseline and after 6 wk of supplementation in the total study population (placebo: $n=32$; hesperidin: $n=33)^{1}$

\begin{tabular}{lccccc}
\hline & \multicolumn{2}{c}{ Placebo } & \multicolumn{2}{c}{ Hesperidin } & $P$ value \\
\cline { 2 - 4 } & Baseline & End & Baseline & End & \\
\hline Total cholesterol, $\mathrm{mmol} / \mathrm{L}$ & $5.7 \pm 0.2$ & $5.6 \pm 0.2$ & $5.5 \pm 0.2$ & $5.4 \pm 0.2$ & 0.752 \\
$\mathrm{LDL} \mathrm{cholesterol,} \mathrm{mmol} / \mathrm{L}$ & $3.6 \pm 0.2$ & $3.6 \pm 0.2$ & $3.5 \pm 0.2$ & $3.4 \pm 0.2$ & 0.585 \\
$\mathrm{HDL} \mathrm{cholesterol,} \mathrm{mmol} / \mathrm{L}$ & $1.5 \pm 0.1$ & $1.5 \pm 0.1$ & $1.5 \pm 0.1$ & $1.5 \pm 0.1$ & 0.884 \\
Triglyceride, $\mathrm{mmol} / \mathrm{L}$ & $1.3 \pm 0.1$ & $1.3 \pm 0.1$ & $1.3 \pm 0.1$ & $1.3 \pm 0.1$ & 0.620 \\
Glucose, $\mathrm{mmol} / \mathrm{L}$ & $5.0 \pm 0.1$ & $5.0 \pm 0.1$ & $4.9 \pm 0.1$ & $5.0 \pm 0.1$ & 0.348 \\
Insulin, $\mathrm{pmol} / \mathrm{L}$ & $58 \pm 21$ & $53 \pm 20$ & $74 \pm 21$ & $81 \pm 20$ & 0.097 \\
OUICKI & $0.42 \pm 0.01$ & $0.23 \pm 0.01$ & $0.34 \pm 0.01$ & $0.42 \pm 0.01$ & 0.225 \\
\hline
\end{tabular}

${ }^{1}$ All values are estimated means \pm SEMs. Differences between placebo and hesperidin were tested with the use of a linear mixed model with correction for baseline values.. QUICKI, quantitative insulin sensitivity check index.

\section{Discussion}

In this trial, the 6-wk effect of hesperidin $2 \mathrm{~S}$ on EF and CVD risk markers were studied in overweight and obese but healthy individuals. To investigate the effect of treatment on EF in 
this healthy population, we performed a stress test and induced reversible ED through the consumption of an HFM. After 6 wk of daily intake of hesperidin 2S, no significant changes in fasted or postprandial FMD were observed. However, a nonsignificant (but nearly significant) downregulation of adhesion molecules sVCAM- 1, sICAM-1, and sP-selectin was observed after 6 wk of hesperidin $2 S$ supplementation compared with after placebo intake. Furthermore, hesperidin $2 S$ tended to reduce both SBP and DBP. The deterioration in FMD after consumption of an HFM was attenuated by hesperidin $2 S$ in a group of subjects with baseline FMD $\geq 3 \%$. In addition, in this subgroup, the downregulation in SVCAM-1 and sICAM- 1 was significant. However, all significant results were no longer significant after correction for multiple testing.

Atherosclerosis is a complex process involving a number of factors and inflammatory cells interacting throughout different stages of development. This process is initiated by circulating plasma LDL entering the subendothelial space in the blood vessel. LDL is oxidized by reactive oxygen species and upregulates adhesion molecules (e.g., sVCAM- 1, sICAM-1, sEselectin and sP-selectin) on the endothelium and induces the expression of chemotactic agents in endothelial cells. ${ }^{29}$ In addition, optimal functioning of the endothelium itself is essential for undisturbed functioning of the cardiovascular system. To study this endpoint, FMD is the gold-standard method for noninvasive EF assessment. ${ }^{30}$ FMD is considered a direct and reliable measure of the vascular reactivity of the macrocirculatory system. ${ }^{31,32}$ In the current study, we aimed to assess the effect of a specific hesperidin $2 S$ formulation in a generally healthy population. Because this population was not screened for impaired FMD variables, it would have been unlikely that major effects on FMD could have been observed in an unchallenged condition. This unlikelihood was confirmed by the fact that no significant effect was observed on basal FMD after 6 wk of daily hesperidin $2 S$ intake. However, after inducing temporary ED through the consumption of an HFM, hesperidin $2 S$ attenuated the postprandial impairment of FMD in a subgroup of subjects with baseline $F M D \geq 3 \%$. The current data support a promising preventive role for hesperidin in mitigating fed-state oxidative stressors that are known to contribute to early, preclinical atherogenesis in subjects with a relatively healthy endothelium. In the literature, one other human intervention trial, to our knowledge, investigated the effect of hesperidin on FMD and showed a significant increase in basal FMD after 3 wk of hesperidin supplementation. The study was conducted in individuals with metabolic syndrome who were specifically included for moderately impaired FMD. ${ }^{13}$ Results from human trials that have examined the effect of other polyphenols on basal FMD have been contradicting, with some trials showing an improvement, ${ }^{28,33,34}$ whereas other trials did not observe any effect ${ }^{35,36}$ after several weeks of supplementation. Only a few studies investigated the effect on postprandial FMD in humans. Results of some studies ${ }^{37-41}$ were in line with ours with some investigators indicating that a change in FMD over time may even be more closely related to a benefit of the intervention and prognosis than of a single FMD measurement. ${ }^{42}$

Plasma adhesion molecule concentrations reflect the degree of $\mathrm{EF}^{25}$ In the current study, a borderline significant downregulation of SVCAM- 1 and sICAM- 1 and a nonsignificant (but nearly significant) downregulation of sP-selectin was observed in the total study population 
after 6 wk of hesperidin $2 S$ intake. In the FMD $\geq 3 \%$ group, the reduction in plasma sVCAM-1 and sICAM-1 was significant. However, after correction for multiple testing, these results were no longer significant. A reduction of sVCAM- 1 and sICAM- 1 may reduce plaque formation, whereas the downregulation of sP-selectin may result in a more-balanced control of platelet aggregation. The combination of both effects may result in a synergistic protection of the endothelium against atherosclerosis. Other human trials that have investigated the effect of hesperidin on adhesion molecules have been scarce. Rizza et al. ${ }^{13}$ showed a downregulation of the adhesion molecule sE-selectin after 3 wk of hesperidin supplementation in metabolic syndrome individuals. However, another study in overweight men did not observe significant differences in circulating adhesion molecules after 4 wk of supplementation with hesperidin. ${ }^{43}$ Human trials that have investigated the effect of other polyphenols on adhesion molecules have shown conflicting results. ${ }^{34,44-48}$ We anticipated seeing the effects of repeated intake of hesperidin for a certain period. The study design allowed us to also assess the effect of a single dosage of hesperidin $2 \mathrm{~S}$. In the FMD $\geq 3 \%$ group, the postprandial downregulation of $\mathrm{sICAM-1}$ was observed after 1 dose of hesperidin $2 \mathrm{~S}$ in response to a fat load. This observation is in line with other literature.47,49-51 The BP-lowering effect of hesperidin, and of polyphenols more generally, has been shown in several human-intervention studies ${ }^{28,34,43,52-54}$ although the effect has not been confirmed in all studies. ${ }^{13,33,45}$ Possible mechanisms by which polyphenol-rich food products lower BP involve an increase in the $\mathrm{NO}$-synthase concentration ${ }^{55.56}$ or the inhibition of the angiotensin-converting enzyme. ${ }^{57,58}$ As previously discussed, the mitigation of fed-state oxidative stressors and the downregulation of molecules by 6 wk of hesperidin $2 S$ use might reflect an improved functionality of the endothelium, which is capable of producing $\mathrm{NO}$, with a consequent vasodilatation and decrease in BP. In this study, a nonsignificant (but nearly significant) reduction in both SBP and DBP was observed in the total study population and in the subgroup. There is a linear relation between BP and mortality from stroke and ischemic heart disease that underscores the importance of BP control. ${ }^{59}$ The hesperidin-induced lowering of SBP $(5 \mathrm{~mm} \mathrm{Hg}$ ) may be of clinical relevance because reductions of this magnitude are associated with a decrease in mortality of $14 \%$ from stroke and $9 \%$ from coronary heart disease..$^{60}$ In addition, we observed a hesperidin-induced reduction of $2 \mathrm{~mm} \mathrm{Hg}$ in DBP, which has been calculated to result in a $17 \%$ decrease in the prevalence of hypertension, a 15\% reduction in risks of stroke and transient ischemic attacks, and a $6 \%$ reduction in risk of coronary heart disease in a general population. ${ }^{61}$

Obesity is typically associated with a wide cluster of metabolic alterations including dyslipidemia and glucose homeostasis disorders. Both in vitro and in vivo studies have shown beneficial effects of hesperidin treatment on cholesterol ${ }^{62-65}$ and glucose metabolism. ${ }^{63,66,67}$ In the current study, supplementation with hesperidin $2 S$ in overweight but otherwise healthy volunteers did not affect systemic glucose, insulin, and lipid concentrations. This finding was not unexpected, because the participants had completely normal values at the start of the trial.

Because we aimed to work with a broad, representative population rather than screening for a specific suboptimal population, we applied a unique test design to evaluate both the acute and b-wk effects of hesperidin 2S. Some potential shortcomings should be considered. First, brachial FMD was used for measuring EF; an extrapolation of our findings to the coronary circulation should be made with caution. However, there has been extensive 
evidence on the correlation of EF in the 2 vascular beds. ${ }^{68,69}$ Second, to assess the postprandial effect of hesperidin 2S, we chose to perform the postprandial FMD $2 \mathrm{~h}$ after intake of the HFM. It is possible that we missed an effect between the time of intake and 2 $h$ after intake. Furthermore, we did not fully control the diets of our participants. We instructed the subjects to maintain their habitual diets during the study period but to abstain from intakes of hesperidin-rich foods. We aimed to assess the effect of hesperidin $2 S$ when supplementing the habitual diet, which is in line with future applications.

In conclusion, 6 wk of hesperidin 2S supplementation in healthy, overweight individuals did not improve basal or postprandial FMD. However, the supplementation appeared to reduce circulating adhesion molecules and tended to lower both SBP and DBP. In a subset of subjects with baseline FMD $\geq 3 \%$, 6 wk of hesperidin $2 S$ intake protected them from a deterioration in FMD after intake of an HFM. In addition, in this subgroup, the reduction in adhesion molecules was significant. Further research to confirm these exploratory findings is warranted. However, these results reflect the potential preventive therapeutic properties of hesperidin 2S. These properties were not observed when looking at the population as a whole, probably because a number of participants had such a deteriorated endothelium that could not be affected by an intervention. Our exploratory findings suggest that hesperidin $2 S$ might be beneficial in individuals with a relatively healthy and, therefore, favorably modifiable endothelium. This possibility underlines the need for early diagnosis and reveals the opportunities for the cardiovascular health benefits of hesperidin $2 S$ in preventive medicine. 


\section{References}

1. Kasprzak JD, Klosinska M, Drozdz J. Clinical aspects of assessment of endothelial function. Pharmacol Rep. 2006;58 Suppl:33-40.

2. Mudau M, Genis A, Lochner A, Striidom H. Endothelial dysfunction: the early predictor of atherosclerosis. Cardiovasc J Afr. 2012;23(4):222-31.

3. Campia U, Tesauro M, Cardillo C. Human obesity and endothelium-dependent responsiveness. $\mathrm{Br} J$ Pharmacol. 2012; 165(3):561-73.

4. Vanhoutte PM, Feletou M, Taddei S. Endothelium-dependent contractions in hypertension. Br J Pharmacol. 2005; 1 44(4):449-58.

5. Johnstone MT, Creager SJ, Scales KM, et al. Impaired endothelium-dependent vasodilation in patients with insulin-dependent diabetes mellitus. Circulation. 1993;88(6): 2510-6.

6. Hsueh WA, Lyon CJ, Quinones MJ. Insulin resistance and the endothelium. Am J Med. 2004; 1 17(2): $109-17$.

7. Forstermann U, Munzel T. Endothelial nitric oxide synthase in vascular disease: from marvel to menace. Circulation. 2006; 1 13(13): 1708-14.

8. Deanfield JE, Halcox JP, Rabelink TJ. Endothelial function and dysfunction testing and clinical relevance. Circulation. 2007; 1 15(10): 1285-95.

9. Jublanc C, Beaudeux JL, Aubart F, et al. Serum levels of adhesion molecules ICAM-1 and VCAM-1 and tissue inhibitor of metalloproteinases, TIMP-1, are elevated in patients with autoimmune thyroid disorders: relevance to vascular inflammation. Nutr Metab Cardiovasc Dis. 201 1;21(10):817-22.

10. Ridker PM, Buring JE, Rifai N. Soluble P-selectin and the risk of future cardiovascular events. Circulation. 200 1; 103(4):491-5.

11. Cermak R, Breves GM. In vitro degradation of the flavonol quercetin and of quercetin glycosides in the porcine hindgut. Arch Anim Nutr. 2006;60(2): 180-9.

12. Erlund I, Meririnne E, Alfthan G, Aro A. Plasma kinetics and urinary excretion of the flavanones naringenin and hesperetin in humans after ingestion of orange juice and grapefruit juice. J Nutr. 2001; 131(2):235-41.

13. Rizza S, Muniyappa R, lantorno $M$, et al. Citrus polyphenol hesperidin stimulates production of nitric oxide in endothelial cells while improving endothelial function and reducing inflammatory markers in patients with metabolic syndrome. J Clin Endocrinol Metab. 201 1;96(5):E782-92.

14. Marder M, Viola H, Wasowski C, et al. 6-methylapigenin and hesperidin: new valeriana flavonoids with activity on the CNS. Pharmacol Biochem Behav. 2003;75(3):537-45.

15. Brand W, Shao J, Hoek-van den Hil EF, et al. Stereoselective conjugation, transport and bioactivity of s- and R-hesperetin enantiomers in vitro. J Agric Food Chem. 2010;58(10):61 19-25.

16. lantorno M, Campia U, Di Daniele N, et al. Obesity, inflammation and endothelial dysfunction. J Biol Regul Homeost Agents. 2014;28(2): 169-76.

17. Vogel RA, Corretti MC, Plotnick GD. Effect of a single high-fat meal on endothelial function in healthy subjects. Am J Cardiol. 1997;79(3):350-4.

18. Gosmanov AR, Smiley DD, Robalino $G$, et al. Effects of oral and intravenous fat load on blood pressure, endothelial function, sympathetic activity, and oxidative stress in obese healthy subjects. Am J Physiol Endocrinol Metab. 2010;299(6):E953-8.

19. Witte DR, Westerink J, de Koning EJ, et al.. Is the association between flow-mediated dilation and cardiovascular risk limited to low-risk populations? J Am Coll Cardiol. 2005; 45: 1987-93.

20. Donald AE, Halcox JP, Charakida M, et al. Methodological approaches to optimize reproducibility and power in clinical studies of flow-mediated dilation. J Am Coll Cardiol. 2008;51:1959-64.

21. Benetos A, Waeber B, Izzo J, et al. Influence of age, risk factors, and cardiovascular and renal disease on arterial stiffness: clinical applications. Am J Hypertens. 2002; 15(12):1 101-8. 
22. Corretti MC, Anderson TJ, Benjamin EJ, et al. Guidelines for the ultrasound assessment of endothelialdependent flow-mediated vasodilation of the brachial artery: a report of the International Brachial Artery Reactivity Task Force. J Am Coll Cardiol. 2002;39(2):257-65.

23. Brevetti G, Martone VD, de Cristofaro T, et al. High levels of adhesion molecules are associated with impaired endothelium-dependent vasodilation in patients with peripheral arterial disease. Thromb Haemost. 2001;85(1):63-6.

24. Clark JL, Zahradka P, Taylor CG. Efficacy of flavonoids in the management of high blood pressure. Nutr Rev. 2015;73(12):799-822.

25. Sankatsing RR, de Groot E, Jukema JW, et al. Surrogate markers for atherosclerotic disease. Curr Opin Lipidol. 2005; 16(4):434-41.

26. Donald AE, Charakida M, Cole TJ, et al. Non-invasive assessment of endothelial function: which technique? J Am Coll Cardiol. 2006;48(9): 1846-50

27. Katz A, Nambi SS, Mather K, et al. Quantitative insulin sensitivity check index: a simple, accurate method for assessing insulin sensitivity in humans. J Clin Endocrinol Metab. 2000;85(7):2402-10.

28. Grassi D, Desideri G, Necozione S, et al. Blood pressure is reduced and insulin sensitivity increased in glucose-intolerant, hypertensive subjects after 15 days of consuming high-polyphenol dark chocolate. J Nutr. 2008; 138(9): 1671-6.

29. Palmefors H, DuttaRoy S, Rundqvist B, Borjesson M. The effect of physical activity or exercise on key biomarkers in atherosclerosis--a systematic review. Atherosclerosis. 2014;235(1):150-61.

30. Frolow M, Drozdz A, Kowalewska A, et al. Comprehensive assessment of vascular health in patients; towards endothelium-guided therapy. Pharmacol Rep. 2015;67(4):786-92.

31. Inaba Y, Chen JA, Bergmann SR. Prediction of future cardiovascular outcomes by flow-mediated vasodilatation of brachial artery: a meta-analysis. Int J Cardiovasc Imaging. 2010;26(6):63 1-40.

32. Ras RT, Streppel MT, Draijer R, Zock PL. Flow-mediated dilation and cardiovascular risk prediction: a systematic review with meta-analysis. Int J Cardiol. 2013;168(1):344-51.

33. Choi EY, Lee H, Woo JS, et al. Effect of onion peel extract on endothelial function and endothelial progenitor cells in overweight and obese individuals. Nutrition. 2015;31(9): 1131 -5.

34. Barona J, Aristizabal JC, Blesso CN, et al. Grape polyphenols reduce blood pressure and increase flowmediated vasodilation in men with metabolic syndrome. J Nutr. 2012; 142(9): 1626-32.

35. Mellen PB, Daniel KR, Brosnihan KB, et al. Effect of muscadine grape seed supplementation on vascular function in subjects with or at risk for cardiovascular disease: a randomized crossover trial. J Am Coll Nutr. 2010;29(5):469-75.

36. Dohadwala MM, Holbrook M, Hamburg NM, et al. Effects of cranberry juice consumption on vascular function in patients with coronary artery disease. Am J Clin Nutr. 201 1:93(5):934-40.

37. Volek JS, Judelson DA, Silvestre R, et al. Effects of carnitine supplementation on flow-mediated dilation and vascular inflammatory responses to a high-fat meal in healthy young adults. Am J Cardiol. 2008; 102(10): 1413-7.

38. Fuentes F, Lopez-Miranda J, Perez-Martinez P, et al. Chronic effects of a high-fat diet enriched with virgin olive oil and a low-fat diet enriched with alpha-linolenic acid on postprandial endothelial function in healthy men. Br J Nutr. 2008; 100(1): 159-65.

39. Plotnick GD, Corretti MC, Vogel RA, et al. Effect of supplemental phytonutrients on impairment of the flowmediated brachial artery vasoactivity after a single high-fat meal. J Am Coll Cardiol. 2003;41(10): 1744-9.

40. Katz DL, Evans MA, Chan W, et al. Oats, antioxidants and endothelial function in overweight, dyslipidemic adults. J Am Coll Nutr. 2004;23(5):397-403.

41. Nakayama H, Tsuge N, Sawada H, Higashi Y. Chronic intake of onion extract containing quercetin improved postprandial endothelial dysfunction in healthy men. J Am Coll Nutr. 2013;32(3): 160-4.

42. Suessenbacher A, Frick M, Alber HF, et al. Association of improvement of brachial artery flow-mediated vasodilation with cardiovascular events. Vasc Med. 2006; 1 1(4):239-44. 
43. Morand C, Dubray C, Milenkovic D, et al. Hesperidin contributes to the vascular protective effects of orange juice: a randomized crossover study in healthy volunteers. Am J Clin Nutr. 20 1 1;93(1):73-80.

44. Basu A, Du M, Sanchez K, et al. Green tea minimally affects biomarkers of inflammation in obese subjects with metabolic syndrome. Nutrition. 201 1;27(2):206-13.

45. Monagas $M$, Khan N, Andres-Lacueva C, et al. Effect of cocoa powder on the modulation of inflammatory biomarkers in patients at high risk of cardiovascular disease. Am J Clin Nutr. 2009:90(5): 1 144-50.

46. Chiva-Blanch G, Urpi-Sarda M, Llorach R, et al. Differential effects of polyphenols and alcohol of red wine on the expression of adhesion molecules and inflammatory cytokines related to atherosclerosis: a randomized clinical trial. Am J Clin Nutr. 2012;95(2):326-34

47. Pacheco YM, Lopez S, Bermudez B, et al. A meal rich in oleic acid beneficially modulates postprandial sICAM- 1 and sVCAM- 1 in normotensive and hypertensive hypertriglyceridemic subjects. J Nutr Biochem. 2008; 19(3):200-5.

48. Schoen C, Schulz A, Schweikart J, et al. Regulatory effects of a fermented food concentrate on immune function parameters in healthy volunteers. Nutrition. 2009;25(5):499-505.

49. Pacheco YM, Bemudez B, Lopez S, et al. Minor compounds of olive oil have postprandial anti-inflammatory effects. Br J Nutr. 2007;98(2):260-3.

50. Vazquez-Agell M, Urpi-Sarda M, Sacanella E, et al. Cocoa consumption reduces NF-kappaB activation in peripheral blood mononuclear cells in humans. Nutr Metab Cardiovasc Dis. 2013;23(3):257-63.

51. Masson CJ, Mensink RP. Exchanging saturated fatty acids for ( $n$ - 6 ) polyunsaturated fatty acids in a mixed meal may decrease postprandial lipemia and markers of inflammation and endothelial activity in overweight men. J Nutr. $2011 ; 141(5): 816-21$.

52. Edwards RL, Lyon T, Litwin SE, et al. Quercetin reduces blood pressure in hypertensive subjects. J Nutr. 2007; $137(11$ 1):2405- 11

53. Basu A, Du M, Leyva MJ, et al. Blueberries decrease cardiovascular risk factors in obese men and women with metabolic syndrome. J Nutr. 2010; 140(9): 1582-7.

54. Novotny JA, Baer DJ, Khoo C, et al. Cranberry juice consumption lowers markers of cardiometabolic risk, including blood pressure and circulating C-reactive protein, triglyceride, and glucose concentrations in adults. J Nutr. 2015; 145(6): 11 185-93.

55. Xu JW, Ikeda K, Yamori Y. Upregulation of endothelial nitric oxide synthase by cyanidin-3-glucoside, a typical anthocyanin pigment. Hypertension. 2004;44(2):217-22.

56. Lazze MC, Pizzala R, Perucca $P$, et al. Anthocyanidins decrease endothelin- 1 production and increase endothelial nitric oxide synthase in human endothelial cells. Mol Nutr Food Res. 2006;50 (1):44-51.

57. Hansen K, Adsersen A, Smitt UW, et al. Angiotensin converting enzyme (ACE) inhibitory flavonoids from Erythroxylum laurifolium. Phytomedicine. 1996;2(4): 313-7.

58. Actis-Goretta L, Ottaviani II, Keen CL, Fraga CG. Inhibition of angiotensin converting enzyme (ACE) activity by flavan-3-ols and procyanidins. FEBS Lett. 2003;555(3):597-600.

59. Lewington S, Clarke R, Qizilbash N, et al, Prospective Studies C. Age-specific relevance of usual blood pressure to vascular mortality: a meta-analysis of individual data for one million adults in 61 prospective studies. Lancet. 2002;360(9349): 1903-13.

60. Whelton PK, He J, Appel LJ, et al. Primary prevention of hypertension: clinical and public health advisory from The National High Blood Pressure Education Program. JAMA. 2002;288(15):1882-8.

61. Cook NR, Cohen J, Hebert PR, et al. Implications of small reductions in diastolic blood pressure for primary prevention. Arch Intern Med. 1995; 155(7):701-9.

62. Bok SH, Lee SH, Park YB, et al. Plasma and hepatic cholesterol and hepatic activities of 3-hydroxy-3-methylglutaryl-CoA reductase and acyl CoA: cholesterol transferase are lower in rats fed citrus peel extract or a mixture of citrus bioflavonoids. J Nutr. 1999; 129:1 182-5.

63. Jung UJ, Lee MK, Park YB, et al. Effect of citrus flavonoids on lipid metabolism and glucose-regulating enzyme mRNA levels in type-2 diabetic mice. Int J Biochem Cell Biol. 2006;38(7): 1 134-45. 
64. Kim HK, Jeong TS, Lee MK, et al. Lipid-lowering efficacy of hesperetin metabolites in high-cholesterol fed rats. Clin Chim Acta. 2003;327(1-2):129-37.

65. Kurowska EM, Spence JD, Jordan J, et al. HDL-cholesterol-raising effect of orange juice in subjects with hypercholesterolemia. Am J Clin Nutr. 2000;72(5): 1095-100

66. Zhang B, Chen T, Chen Z, et al. Synthesis and anti-hyperglycemic activity of hesperidin derivatives. Bioorg Med Chem Lett. 2012;22(23):7194-7.

67. Jung UJ, Lee MK, Jeong KS, Choi MS. The hypoglycemic effects of hesperidin and naringin are partly mediated by hepatic glucose-regulating enzymes in C57BL/KsJ-db/db mice. J Nutr. 2004;134(10):2499503.

68. Anderson TJ, Uehata A, Gerhard MD, et al. Close relation of endothelial function in the human coronary and peripheral circulations. J Am Coll Cardiol. 1995;26(5):1235-41.

69. Flammer AJ, Luscher TF. Three decades of endothelium research: from the detection of nitric oxide to the everyday implementation of endothelial function measurements in cardiovascular diseases. Swiss Med Wkly. 2010; 140:w13122. 
Supplemental material

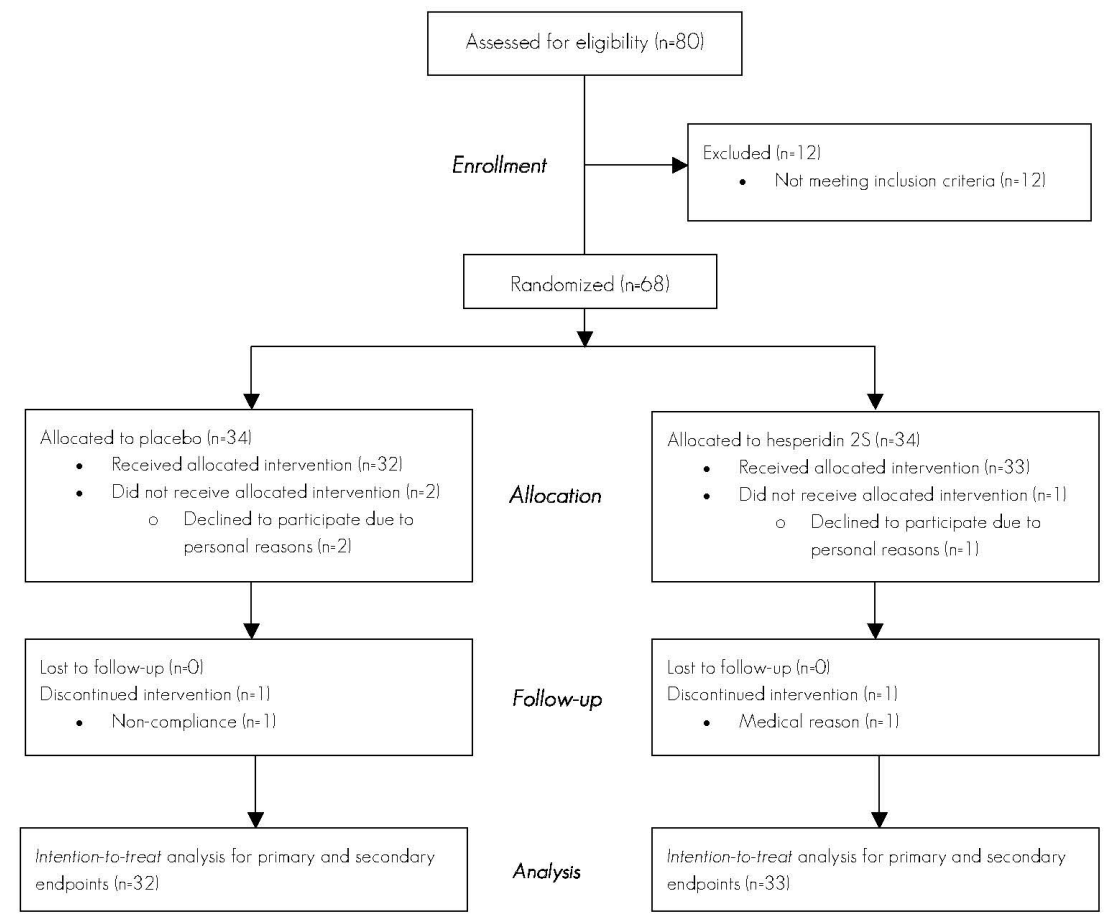

Figure S6.1 CONSORT flow diagram. 
$9 \hat{\xi}$ 


\section{chapter 7}

Effect of hesperidin 2S supplementation on systemic and intestinal metabolic parameters in subjects at risk for metabolic syndrome: a randomized controlled trial Salden BN, Troost FJ, Possemiers S, Stevens YR, Masclee AA 


\section{Abstract}

\section{Background}

The intestinal ecosystem plays an important role in host homeostasis. Obesity-related changes in gut microbiota composition and microbial activity may promote the development of metabolic disturbances, such as insulin resistance. Hesperidin, a citrus polyphenol with antioxidant and other biological properties, is poorly absorbed in the small intestine and therefore reaches the colon, where it interacts with the colonic microbiota. In line with this, it may therefore exert beneficial effects on gastrointestinal $(G \mid)$ and systemic metabolic disturbances.

\section{Objective}

Primary aim of the study was to investigate the effect of 12 wks hesperidin $2 S$ supplementation on glucose regulation. Secondly, we aimed to investigate the effect of hesperidin $2 S$ on modulation of the intestinal environment and on systemic metabolic parameters in subjects with features of metabolic syndrome (MetS).

\section{Design}

In this randomized, double-blind, placebo-controlled study, 50 individuals were randomly assigned to groups receiving hesperidin $2 \mathrm{~S}$ (Citrus sinensis extract; $450 \mathrm{mg} / \mathrm{d}$ ) or placebo for $12 \mathrm{wks}$. Measurements at baseline and after 12 wks intervention included oral glucose tolerance test (OGTT), blood lipids, blood pressure (BP), fecal short-chain fatty acids (SCFA) and fecal calprotectin. Additionally, after 6 wks intervention fasting glucose, insulin, blood lipids and BP were measured.

Results

No significant changes in fasting glucose and insulin and in $\mathrm{AUC}_{0-120 \mathrm{~min}}$ glucose and $\mathrm{AUC}_{0-120 \mathrm{~min}}$ insulin were observed after 12 wks hesperidin intake, compared to placebo. While hesperidin $2 S$ did not quantitatively affect total fecal SCFA concentrations, it beneficially altered the SCFA profile by inducing a shift from acetate to butyrate $(P=0.020)$. Fecal calprotectin showed a near-significant reduction after 12 wks hesperidin supplementation, compared to placebo $(P=0.058)$. No significant changes in blood lipids and BP were observed after hesperidin intake, compared to placebo.

\section{Conclusions}

Twelve weeks consumption of hesperidin 2S beneficially altered the fecal SCFA profile and calprotectin concentrations, without affecting glucose regulation, blood lipids or BP in subjects with features of MetS. 


\section{Introduction}

The rapidly growing prevalence of obesity, and its metabolic and gastrointestinal (GI) consequences, is a major public health concern. 'Hence, developing new preventive and therapeutic strategies is important. In this respect, the use of polyphenols appears to be an attractive approach as these compounds are potent antioxidants exerting anti-inflammatory, anti-diabetic, anti-hypertensive and antihyperlipidemic effects. ${ }^{2}$ Hesperidin (hesperetin-7-Orutinoside), a polyphenol abundantly present in the peels of citrus fruits, is such a promising compound. It showed to decrease the gene expression of cyclooxygenase-2, demonstrating its anti-inflammatory potential. ${ }^{3}$ Furthermore, it significantly improved hyperglycemia by increasing glucose utilization in a diabetic mice model. ${ }^{4}$ In humans, three weeks daily supplementation of hesperidin caused a trend towards improving insulin resistance in MetS patients. ${ }^{5}$ Additionally, it showed to exert lipid lowering activity in hyperlipidemic rats ${ }^{6}$ and hyperlipidemic humans. 'Also blood pressure (BP) was reduced after hesperidin treatment, both in spontaneously hypertensive rats $^{8}$ and in humans. ${ }^{9}$ Obesity and metabolic syndrome (MetS) have also been associated with changes in gut health, mainly caused by alterations in the composition of gut microbiota, microbial and metabolic activity. 10,11 Interestingly, recent data suggest that different polyphenolic compounds, including hesperidin, are able to modulate the gut microbiome structure and function, thereby beneficially affecting gut health. ${ }^{12,13}$ As hesperidin is poorly absorbed in the small intestine, a major fraction reaches the colon where it indeed can interact with the intestinal microbiota. ${ }^{14}$ However, low solubility of hesperidin limits its bioavailability and biological activity in vivo, including local effects in the intestine. ${ }^{15}$ Furthermore, while natural products contain high concentrations of the most active form of hesperidin (hesperitin-7-O-rutinoside 2S), typical hesperidin extracts on the market mainly contain the less active $2 \mathrm{R}$ enantiomer. ${ }^{16}$

This study was conducted to investigate the effect of 12 wks supplementation of a hesperidin 2S extract (Cordiart), a hesperidin enantiomer which has an improved bioavailability and biological activity, on $G$ and on systemic metabolic parameters in individuals with features of MetS. We hypothesized that 12 wks hesperidin 2S supplementation will exert a) gastrointestinal effects with changes in metabolic activity of gut microbiota and intestinal inflammation, and b) systemic effects consisting of improvement in glucose regulation, lowering of systolic and diastolic BP, and improvement of blood lipid profile in individuals with features of MetS. Primary aim of our study was to investigate the effect of 12 wks supplementation of hesperidin $2 S$ on glucose regulation, assessed via an oral glucose tolerance test (OGTT). Secondary, we aimed to investigate: 1) gastrointestinal effects, by measuring fecal short-chain fatty acids (SCFA) concentrations and fecal calprotectin and 2) systemic effects, by measuring BP and blood lipids. 


\section{Materials and methods}

The study was approved by the Medical Ethics Committee of the Maastricht University Medical Center (MUMC+) and conducted in full accordance with the principles of the Declaration of Helsinki of 1975 as amended in 2013 and with the Dutch Regulations on Medical Research involving Human Subjects (WMO, 1998). The study was performed at the MUMC+ from February 2015 to November 2015. All participants gave written informed consent before participation. The trial has been registered in the Clinical Trials register (NCTO2610491).

\section{Subjects}

Volunteers were recruited by advertising in the local media. Inclusion criteria were age between 18 and 66 yrs, and two of the following: waist circumference $>102 \mathrm{~cm}$ (males) or $>88 \mathrm{~cm}$ (females), abnormal fasting serum triglycerides $\geq 1.7 \mathrm{mmol} / /$, abnormal fasting serum $\mathrm{HDL}$-cholesterol $\leq 1.0 \mathrm{mmol} / /$ (males) or $\leq 1.3 \mathrm{mmol} / \mathrm{l}$ (females), systolic BP (SBP) $\geq 130 \mathrm{mmHg}$ and/or diastolic BP (DBP) $\geq 85 \mathrm{mmHg}$, elevated fasting plasma glucose $\geq 6.1 \mathrm{mmol} / \mathrm{I}^{17}$ Exclusion criteria were: diabetes mellitus (defined as fasting plasma glucose of $\geq 7 \mathrm{mmol} / \mathrm{l}$ ); any medical condition that might interfere with the study and might jeopardise the health status of the participant; smoking; abuse of alcohol (>20 alcoholic units / week) and drugs; no consistently stable body weight for at least 3 months $( \pm 3 \mathrm{~kg})$; plans to lose weight or following energy restriction diet during study period; use of medication / vitamin-, mineralor antioxidant supplements; use of antibiotics in the 90 days prior to start of the study; pregnancy and lactation; history of any side effects towards the intake of flavonoids or citrus fruits; failure to comply with prohibited intake of hesperidin rich food products. During the study period, subjects consumed their habitual diet. During the trial intake of hesperidinrich food products was prohibited.

\section{Design and intervention}

This study was designed as a randomized, placebo-controlled, double-blind, parallel group study (Online Supplemental Material Figure S7.1). Each subject underwent 3 test days. Participants were randomly assigned in a double-blind fashion to one of the two intervention arms: hesperidin $2 \mathrm{~S}$ or placebo (cellulose). An independent person generated the randomization list, using a computerized procedure. All participants and investigators remained blind to treatment until all analyses had been completed. From 4 days prior to start of the study until the last test day, participants were instructed to refrain from consuming any hesperidin rich food products. Furthermore, participants were requested to 
abstain from strenuous physical exercise and consumption of alcohol on the day before each test day. Assessments took place in a quiet, temperature-controlled $\left(20-24^{\circ} \mathrm{C}\right.$ ) room. After an overnight fast, subjects collected a fecal sample on the first test day. Subsequently, anthropometric measurements (height, body weight, waist-to-hip circumference) were performed and BP was measured. Subsequently, an intravenous catheter was inserted into the antecubital vein of the arm and blood samples were collected. Next, the participant was instructed to ingest $75 \mathrm{~g}$ glucose (OranGluc75, Novolab, Geraardsbergen, Belgium) within 5 minutes. Blood samples for glucose and insulin concentrations were collected before and at 30, 60, 90 and 120 minutes after ingestion of the glucose load, respectively. At the end of the test day the intravenous catheter was removed and participants received the study products for the first 6 wks. After 6 wks of daily supplementation, test day 2 was organized. After an overnight fast, BP was measured and blood samples were taken. Again, study products for another 6 wks were provided. Test day 3 was conducted at the end of the supplementation period of 12 wks. Measurements were identical to the measurements performed on test day 1. To assess compliance, participants were asked to save the empty and (partly) full blister packs and to return these at the last visit. Also, in the first and last week of the study period participants were asked to fill out a 3-day food record to assess whether they maintained their dietary habits throughout the study period.

\section{Hesperidin 2S and placebo}

Hesperidin 2S $(450$ mg, supplied as 500 mg Cordiart, BioActor BV, Maastricht, The Netherlands) was extracted from the Citrus sinesis immature fruit, containing both the $S$ and R enantiomers in the natural 4:1 S:R ratio of hesperidin. Cellulose $1500 \mathrm{mg}$ microcrystalline cellulose; Aminolabs; Hasselt; Belgium) served as placebo. The study products were formulated into capsules, each containing $250 \mathrm{mg}$ study product or placebo. Subjects were asked to ingest two capsules each morning with $200 \mathrm{ml}$ water, just before consuming breakfast, for 12 wks.

\section{Fecal SCFA determination}

Fecal samples were collected by the participants and stored at $-20^{\circ} \mathrm{C}$ until handed in at the study site. For SCFA analysis, aliquots of approximately $1 \mathrm{~g}$ of fecal samples were diluted and homogenized with $6 \mathrm{ml}$ demineralized water. After removal of the particulate material by centrifugation $(10 \mathrm{~min}, 500 \times \mathrm{g})$, the supernatants were stored at $-20^{\circ} \mathrm{C}$ prior to analysis. ${ }^{18}$ SCFA concentrations in the supernatants were determined using gas chromatography with flame ionization detector. Total SCFA was defined as the sum of acetate, propionate, butyrate, isobutyrate, valerate, isovalerate, caproate and isocaproate. The analysis was identical to the SCFA analysis described by Possemiers et al. ${ }^{19}$ 


\section{Fecal calprotectin}

Fecal calprotectin concentration levels were determined using an ELISA for human calprotectin (Hycult Biotechnology, Uden, Netherlands), according to the manufacturer's protocol. Briefly, $5 \mathrm{ml}$ of extraction buffer $(0.1 \mathrm{M}$ Tris, $0.15 \mathrm{M} \mathrm{NaCl}, 1.0 \mathrm{M}$ Urea, $10 \mathrm{mM}$ $\mathrm{CaCl}_{2} \mathrm{H}_{2} \mathrm{O}, 0.1 \mathrm{M}$ Citric Acid and 0.5\% BSA, pH 8) was added to $100 \mathrm{mg}$ of feces. The suspension was vortexed and shaken for 30 minutes with a rotating shaker at $4^{\circ} \mathrm{C}$. After shaking, the suspension was centrifuged at $10,000 \mathrm{rpm}$ for 20 minutes at $4^{\circ} \mathrm{C}$. The supernatant was stored at $-20^{\circ} \mathrm{C}$ until analysis.

\section{Glucose / insulin metabolism}

\section{Oral glucose tolerance test (OGTT)}

Blood samples for measuring glucose and insulin concentrations were collected before and at 30, 60, 90 and 120 minutes after ingestion of the glucose load, respectively. Serum concentrations of glucose were measured by spectrophotometry (cobas 6000 analyzer series, Roche Diagnostics, Indianapolis, USA). Plasma insulin was determined using a luminescence-enhanced immune-enzymatic assay (Immulite 2000 immunoassay system, Siemens Healthcare, Erlangen, Germany). Results are presented as glucose and insulin 120-min AUC ( $\mathrm{AUC}_{0-120 \mathrm{~min}}$. The $\mathrm{AUC}_{0-120 \mathrm{~min}}$ was calculated by the trapezoidal method.

\section{Homeostasis model assessment of insulin resistance (HOMA-IR)}

OGTT values were used for calculating HOMA-IR index, an index of insulin resistance. ${ }^{20}$ It was calculated as the product of fasting plasma glucose (mmol//) and insulin (mU/I) concentrations divided by 22.5 .

\section{Quantitative insulin sensitivity check index (QUICKI)}

OGTT values were also used for calculating the QUICKI, an index of insulin sensitivity. ${ }^{21}$ It was calculated as: 1 / (log insulin $\mathrm{O} \mathrm{h}+\log$ glucose $\mathrm{O} \mathrm{h})$.

Insulin sensitivity index (ISI) - Matsuda ${ }^{22}$

OGTT values were used for calculation the composite ISI, an index of insulin sensitivity. It was calculated as: 10,000 / ( $\left(\text { glucose } 0 \mathrm{~h}{ }^{*} \text { insulin } \mathrm{O} \mathrm{h}\right)^{*}$ V(glucose mean ${ }^{*}$ insulin mean)). 


\section{Blood lipids}

Blood samples were collected at baseline, after 6 wks supplementation and at completion of the study at 12 wks. Serum concentrations of total cholesterol, LDL- and HDL-cholesterol and triglycerides were measured by spectrophotometry (cobas 6000 analyzer series, Roche Diagnostics, Indianapolis, USA).

\section{Blood pressure measurements}

BP was monitored using a semi-continuous BP monitoring device 1 Omron, Hoofddorp, The Netherlands) at the upper left arm. On each occasion, after 30 minutes of rest in the supine position, 4 consecutive BP measurements were performed. The first measurement was discarded and the remaining 3 measurements were averaged. Measurements took place at baseline, after 6 wks supplementation and at the end of the study period.

\section{Statistics}

The primary outcome of the study was the effect of 12 wks hesperidin $2 S$ supplementation on glucose regulation, assessed via an OGTT, compared to placebo. Secondly, we investigated the effects of 12 wks hesperidin $2 S$ supplementation on local $G$ parameters: fecal SCFA concentrations and fecal calprotectin, and on systemic metabolic parameters: blood lipids and BP. Sample size was determined for the primary outcome of the study, using a significance level alpha $=0.05$ and a power of $80 \%$. Based on a study investigating the effect of polyphenols on insulin sensitivity in overweight individuals, ${ }^{23}$ we calculated that a sample size of 50 subjects would be required to detect a difference in ISI index of $0.98 \pm$ $1.2 \%$ (mean \pm SD). Baseline characteristics are presented as mean (SD) for numerical variables and number (\%) for categorical variables. Differences in fecal SCFA, fecal calprotectin, glucose regulation, blood lipids and BP between intervention groups (hesperidin $2 \mathrm{~S}$ and placebo) were assessed using mixed models with group (placebo and hesperidin 2S), time (0,6 wks and 12 wks) and group*time as fixed factors, where an unstructured covariance structure was used for repeated measures. The linear mixed model accounts for the correlation between repeated measures and missing data, where a likelihood approach was used assuming data missing at random. The estimated means and SEM obtained from this model were presented for each group and each time point. Twosided P-values $\leq 0.05$ were considered statistically significant. Statistical analysis was performed using IBM SPSS Statistics for Windows (version 21.0, Armonk, NY, USA). 


\section{Results}

\section{Participants}

Initially, 53 healthy volunteers were enrolled in the study, of which 50 completed the entire study protocol. Three participants were included and randomized to a treatment but never started participation due to private reasons. Baseline characteristics of the study participants are presented in Table 7.1.

Table 7.1 Baseline characteristics of study participants.'

\begin{tabular}{lccc}
\hline & $\begin{array}{c}\text { Total population } \\
(n=50)\end{array}$ & $\begin{array}{c}\text { Placebo } \\
(n=27)\end{array}$ & $\begin{array}{c}\text { Hesperidin 2S } \\
(n=23)\end{array}$ \\
\hline Age, $y r$ & $51 \pm 13^{2}$ & $50 \pm 13$ & $52 \pm 11$ \\
Sex, M/F & $18 / 32$ & $9 / 18$ & $9 / 14$ \\
WHR & $0.93 \pm 0.07$ & $0.91 \pm 0.07$ & $0.94 \pm 0.07$ \\
BMl, $\mathrm{kg} / \mathrm{m}^{2}$ & $30.8 \pm 3.80$ & $31.4 \pm 4.20$ & $30.0 \pm 3.20$ \\
\hline
\end{tabular}

${ }^{1}$ WHR, waist-to-hip-ratio. BMI, body mass index. ${ }^{2}$ Mean \pm SD (all such values).

\section{Fecal SCFA}

Data on fecal SCFA concentrations are shown in Table 7.2. Twelve wks daily intake of hesperidin $2 S$ did not significantly change fecal concentrations of total SCFA, branched SCFA, and of acetate, propionate and butyrate, compared to placebo. However, the butyrate proportion of the total SCFA significantly increased after 12 wks hesperidin 2S intake $(P=0.031)$. Furthermore, the butyrate over acetate ratio significantly increased in the hesperidin $2 \mathrm{~S}$ group, compared to placebo $(P=0.020)$.

\section{Fecal calprotectin}

Fecal calprotectin showed a trend towards a reduction after 12 wks hesperidin 2S supplementation, compared to placebo ( $P=0.058$, Table 7.2).

\section{Glucose / insulin metabolism}

Baseline glucose and insulin concentrations were all within normal blood value ranges. No significant differences were observed in $A \cup C_{0-120 m i n}$ for both glucose and insulin between intervention groups (Table 7.3). Neither were significant effects of the intervention found in glucose and insulin concentrations at baseline and at $30 \mathrm{~min}, 60 \mathrm{~min}, 90 \mathrm{~min}$ and $120 \mathrm{~min}$ after glucose load, respectively (data not shown). Consistent with these findings, hesperidin 
$2 S$ supplementation did not improve insulin sensitivity or reduce insulin resistance after 6 or 12 wks supplementation, compared to placebo.

Table 7.2 Gastrointestinal parameters at baseline and after 12 wks supplementation with placebo or hesperidin 2S.?

\begin{tabular}{|c|c|c|c|c|c|}
\hline & \multicolumn{2}{|c|}{ Placebo } & \multicolumn{2}{|c|}{ Hesperidin $2 \mathrm{~S}$} & \multirow[t]{2}{*}{$P$ value } \\
\hline & Baseline & 12 wks & Baseline & 12 wks & \\
\hline \multicolumn{6}{|l|}{ Proteins } \\
\hline Calprotectin (ng/ml) & $32.9 \pm 7.8^{2}$ & $42.5 \pm 7.6$ & $47.5 \pm 8.7$ & $37.1 \pm 8.4$ & 0.058 \\
\hline \multicolumn{6}{|l|}{ Short-chain fatty acids } \\
\hline Total ( $\mu \mathrm{mol} / \mathrm{g}$ feces) & $59.8 \pm 5.1$ & $68.2 \pm 5.9$ & $72.9 \pm 5.5$ & $79.4 \pm 6.4$ & 0.806 \\
\hline Branched ( $\mu \mathrm{mol} / \mathrm{g}$ feces) & $3.1 \pm 0.3$ & $3.2 \pm 0.4$ & $3.6 \pm 0.4$ & $3.9 \pm 0.4$ & 0.807 \\
\hline Acetate ( $\mu \mathrm{mol} / \mathrm{g}$ feces) & $32.9 \pm 3.0$ & $38.4 \pm 3.4$ & $39.8 \pm 3.3$ & $42.9 \pm 3.6$ & 0.625 \\
\hline Propionate ( $\mu \mathrm{mol} / \mathrm{g}$ feces) & $11.1 \pm 1.3$ & $12.9 \pm 1.6$ & $13.9 \pm 1.4$ & $14.1 \pm 1.7$ & 0.465 \\
\hline Butyrate ( $\mu \mathrm{mol} / \mathrm{g}$ feces) & $10.4 \pm 1.1$ & $10.9 \pm 1.3$ & $12.4 \pm 1.2$ & $15.0 \pm 1.4$ & 0.249 \\
\hline Acetate / total SCFA ratio & $0.61 \pm 0.01$ & $0.62 \pm 0.01$ & $0.61 \pm 0.01$ & $0.60 \pm 0.02$ & 0.079 \\
\hline Propionate / total SCFA ratio & $0.20 \pm 0.01$ & $0.20 \pm 0.01$ & $0.21 \pm 0.01$ & $0.20 \pm 0.01$ & 0.812 \\
\hline Butyrate / total SCFA ratio & $0.19 \pm 0.01$ & $0.18 \pm 0.01$ & $0.18 \pm 0.01$ & $0.20 \pm 0.01$ & 0.031 \\
\hline Butyrate / acetate ratio & $0.32 \pm 0.02$ & $0.29 \pm 0.02$ & $0.31 \pm 0.03$ & $0.35 \pm 0.02$ & 0.020 \\
\hline
\end{tabular}

1 Differences between placebo and hesperidin tested with linear mixed model with correction for baseline values. $P$ value represents the $\mathrm{p}$-value for the analysis of baseline versus $12 \mathrm{wks}$ intervention between placebo and hesperidin $2 \mathrm{~S}$. ${ }^{2}$ Estimated mean \pm SEM (all such values).

\section{Blood lipids}

Baseline total cholesterol, LDL- and HDL-cholesterol and triglycerides were all within normal blood value ranges. These parameters were not significantly altered by 6 or 12 wks supplementation with hesperidin 2S, compared to placebo (Table 7.3).

\section{Blood pressure}

Results regarding the effect of hesperidin $2 \mathrm{~S}$ administration on BP are given in Table 7.3. No significant changes in SBP, DBP and HR were observed after 6 or after 12 wks supplementation between intervention groups. 


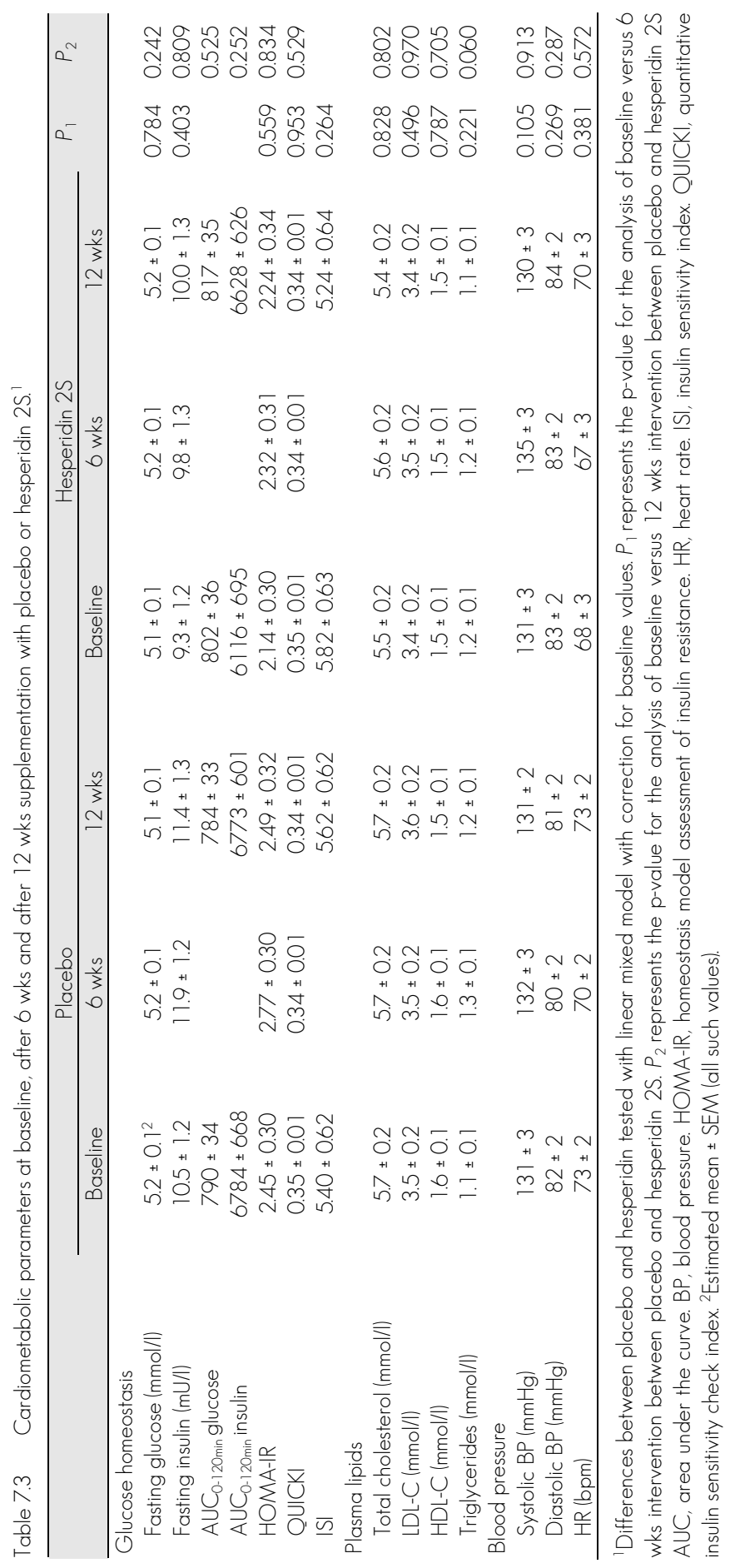




\section{Discussion}

The effects of hesperidin $2 S$ on glucose regulation, local $G$ parameters and systemic metabolic parameters were assessed in subjects with features of MetS. After 12 wks daily intake of hesperidin 2S, no significant changes were observed in glucose regulation, compared to placebo. Interestingly, although the total SCFA production did not change, hesperidin $2 S$ significantly altered the SCFA profile by inducing a shift from acetate to butyrate. Furthermore, hesperidin $2 S$ supplementation resulted in a reduction in fecal calprotectin that just failed to reach statistical significance. No differences in blood lipids or in BP were observed between the intervention groups.

It has been suggested that dietary polyphenols lower the risk of developing DM2. Several mechanisms of action have been described explaining through which the blood glucose lowering effects of hesperidin are mediated. First, polyphenols were shown to inhibit the digestion of dietary carbohydrates by inhibition of key enzymes involved in carbohydrate digestion. ${ }^{24}$ Second, absorption of glucose in the intestine can be impeded by polyphenols. ${ }^{25}$ Third, enhanced insulin-mediated tissue uptake of glucose has been observed with different polyphenols. ${ }^{26}$ Fourth, another mechanism includes the potential of polyphenols to protect against cytokines induced pancreatic $\beta$-cell damage, thereby maintaining insulin secretion. ${ }^{27}$ Additionally, by increasing hepatic glucokinase activity, which augments glucose utilization, and by suppressing hepatic glucose production (i.e. gluconeogenesis) antidiabetic effects can be exerted by several polyphenols. ${ }^{28}$ Finally, polyphenol-mediated modulation of inflammation was shown to improve insulin sensitivity in obese mice and rats. ${ }^{29}$

Another mechanism through which polyphenols were shown to induce hypoglycemic effects is by modulating gut microbiota composition and their metabolic activity. ${ }^{13,30}$ It has become evident that the gut microbiota fulfill an important role in host homeostasis, being involved in many processes such as metabolism of nutrients and drugs, regulation of several metabolic pathways, maintaining epithelial integrity, modulating $\mathrm{Gl}$ motility, stimulation and maturation of both systemic and mucosal immunity, and production of vitamins and micronutrients. ${ }^{31}$ SCFA are produced by bacterial fermentation of mainly undigested dietary carbohydrates. Many of the beneficial physiological properties of the gut microbiota can be attributed to the production of SCFA as these metabolites showed to exert antioxidant, anti-inflammatory and immune modulating effects. ${ }^{32}$ SCFA are able to bind and activate G-protein-coupled receptors in gut epithelial cells. Activation of these receptors induces gut hormone secretion, such as glucagon-like peptide (GLP)-1, GLP-2 and peptide YY (PYY), which increase insulin sensitivity and decrease inflammation. ${ }^{33}$ It has also been suggested that SCFA are able to modulate insulin sensitivity by altering fatty acid flux. Lowering postprandial free fatty acids (FFA) appeared to improve insulin sensitivity and insulin secretion. ${ }^{34}$ The SCFA butyrate beneficially affects oxidative stress in the colonic mucosa, by increasing expression levels of the antioxidant glutathione and decreasing expression levels of uric acid, a predictor in the 
development of insulin resistance ${ }^{35}$ In humans, increasing intestinal butyrate-producing microbes concomitantly improved insulin sensitivity. ${ }^{36}$

In this intervention study, we measured fecal SCFA concentrations to assess microbial activity. We did not observe differences in the quantity of acetate, propionate or butyrate production between the intervention groups. However, we did observe a hesperidininduced alteration in SCFA profile. A significant shift from acetate towards the healthpromoting SCFA butyrate was identified, possibly via an intestinal stimulation of butyrateproducing bacteria. This finding is in line with recent evidence, suggesting that a number of polyphenols and their metabolites are able to influence microbiota composition and microbial activity. ${ }^{12,13}$ Butyrate not only improves insulin sensitivity, but also serves as an energy source for epithelial cells, exerts anticarcinogenic, anti-inflammatory and antioxidant effects, and is essential in maintaining the colonic defence barrier. ${ }^{37}$ We did not observe any change in glucose regulation (both circulating concentrations of glucose and insulin) in the present study. It should be noted that virtually all participants had normal fasting blood glucose values and OGTT values at start of the trial. Results might have been different when glucose-intolerant subjects had been studied. However, even in glucose-intolerant subjects controversial results have been reported. Dark chocolate polyphenols showed to improve insulin sensitivity, ${ }^{38}$ while epigallocatechin-3-gallate supplementation did not. ${ }^{39}$

Calprotectin, a protein secreted by neutrophils infiltrating the intestinal mucosa, is considered a marker of whole gut inflammation. Elevated concentrations of fecal calprotectin are found in patients with chronic intestinal inflammation such as inflammatory bowel disease, but also in obese individuals. ${ }^{40}$ We found fecal calprotectin levels within the normal range, but higher than those reported in healthy controls. After the intervention, fecal calprotectin was reduced in the hesperidin $2 S$ treated group vs. placebo, although this difference just failed to reach statistical significance. It is tempting to speculate on the mechanism of this reduction: whether it is related to changes in gut microbiota and gut metabolism. ${ }^{41}$ It has previously been shown that pre- and probiotics can reduce fecal calprotectin levels, probably through alterations in the gut microbiome. ${ }^{42,43}$

The presence of dyslipidemia is common in obesity. ${ }^{44}$ Hesperidin is known to lower blood lipids in vitro and in vivo, possibly by affecting enzymes involved in lipogenesis, fatty acid biosynthesis, triglyceride synthesis, cholesterol synthesis and cholesterol absorption, and by facilitating fecal triglyceride and cholesterol excretion. ${ }^{28,45-47}$ Here, we did not observe alterations in systemic lipid concentrations after 6 or 12 wks hesperidin $2 S$ intake. This finding was not unexpected as participants had completely normal values at start of the trial. In the present study, hesperidin 2S did not improve systolic or diastolic BP. Most of the studies reporting a polyphenol-induced BP reduction have included hypertensive subjects, while in our study BP values at baseline were within normal ranges. Putative methods by which polyphenols lower BP include increasing nitric oxide synthase concentrations ${ }^{48}$ or 
inhibiting angiotensin-converting enzyme. ${ }^{49}$ Hesperidin and other polyphenols showed to have the potential to reduce BP in vivo, 9,38 although other studies have not observed this. 5,50 A few limitations of our study should be considered. First, we used the OGTT, a surrogate marker for insulin sensitivity instead of the euglycemic hyperinsulinemic clamp, which is considered the gold standard method. However, that method is an expensive, time consuming, labor intensive method requiring an experienced operator. Second, to assess the microbiota fermentation capacity we measured fecal SCFA concentrations. As these metabolites are efficiently absorbed from the gut - with only $5 \%$ to $10 \%$ being excreted via the feces - they may not accurately reflect the exact intraluminal SCFA concentrations. However, previous studies have shown that the ratio between the respective SCFA concentrations, measured in feces and colon, is comparable. ${ }^{51}$ Thus, changes in fecal SCFA concentration reflect relative changes in intestinal concentrations of the separate SCFA. Furthermore, although we included subjects at risk for MetS, most baseline parameters were already within normal ranges at start of the study. Inducing a systemic beneficial effect in a generally healthy population through a dietary intervention is challenging. Finally, we did not fully control the diet of our participants. We instructed them to maintain their habitual diet during the study period, but to abstain from intake of hesperidin-rich foods. We aimed to assess the effect of hesperidin $2 S$ when supplementing the habitual diet, which is in line with future applications.

In conclusion, 12 weeks oral supplementation with hesperidin 2S did not significantly influence glucose regulation in subjects with features of MetS. Interestingly, it modulated the $\mathrm{Gl}$ environment as reflected by a shift in fermentation (SCFA) profile from acetate to butyrate, and a near-significant reduction in fecal calprotectin levels. These changes were not accompanied with changes in blood lipids or BP. These results reinforce the hypothesis that polyphenolic compounds are able to modulate gut microbiota composition and function, thereby promoting gut and host health. 


\section{References}

1. Eckel RH, Alberti KG, Grundy SM, Zimmet PZ. The metabolic syndrome. Lancet 2010;375(9710): 181-3.

2. Cherniack EP. Polyphenols: planting the seeds of treatment for the metabolic syndrome. Nutrition 201 1;27(6):6 17-23.

3. Hirata A, Murakami $Y$, Shoji $M$, et al. Kinetics of radical-scavenging activity of hesperetin and hesperidin and their inhibitory activity on COX-2 expression. Anticancer Res 2005;25(5):3367-74.

4. Jung UJ, Lee MK, Jeong KS, Choi MS. The hypoglycemic effects of hesperidin and naringin are partly mediated by hepatic glucose-regulating enzymes in C57BL/KsJ-db/db mice. J Nutr 2004;134(10): 2499-503.

5. Rizza S, Muniyappa R, lantorno $M$, et al. Citrus polyphenol hesperidin stimulates production of nitric oxide in endothelial cells while improving endothelial function and reducing inflammatory markers in patients with metabolic syndrome. J Clin Endocrinol Metab 201 1;96(5):E782-92.

6. Monforte MT, Trovato A, Kirjavainen S, et al. Biological effects of hesperidin, a Citrus flavonoid. (note II): hypolipidemic activity on experimental hypercholesterolemia in rat. Farmaco 1995;50(9):595-9.

7. Miwa Y, Mitsuzumi H, Sunayama T, et al. Glucosyl hesperidin lowers serum triglyceride level in hypertriglyceridemic subjects through the improvement of very low-density lipoprotein metabolic abnormality. J Nutr Sci Vitaminol (Tokyo) 2005;51(6):460-70.

8. Yamamoto M, Suzuki A, Hase T. Short-term effects of glucosyl hesperidin and hesperetin on blood pressure and vascular endothelial function in spontaneously hypertensive rats. J Nutr Sci Vitaminol (Tokyo) 2008; 54(1):95-8.

9. Morand C, Dubray C, Milenkovic D, et al. Hesperidin contributes to the vascular protective effects of orange juice: a randomized crossover study in healthy volunteers. Am J Clin Nutr 201 1:93( 1):73-80.

10. Musso G, Gambino R, Cassader M. Interactions between gut microbiota and host metabolism predisposing to obesity and diabetes. Annu Rev Med 201 1;62:361-80.

11. Tilg H, Kaser A. Gut microbiome, obesity, and metabolic dysfunction. J Clin Invest 201 1; 121(6): $2126-32$.

12. Unno T, Hisada T, Takahashi S. Hesperetin Modifies the Composition of Fecal Microbiota and Increases Cecal Levels of Short-Chain Fatty Acids in Rats. J Agric Food Chem 2015;63(36):7952-7.

13. Queipo-Ortuño MI, Boto-Ordóñez M, Murri M, et al. Influence of red wine polyphenols and ethanol on the gut microbiota ecology and biochemical biomarkers. Am J Clin Nutr 2012;95(6):1323-34.

14. Possemiers S, Bolca S, Verstraete W, Heyerick A. The intestinal microbiome: a separate organ inside the body with the metabolic potential to influence the bioactivity of botanicals. Fitoterapia 201 1:82(1):53-66.

15. Erlund I, Meririnne E, Alfthan G, Aro A. Plasma kinetics and urinary excretion of the flavanones naringenin and hesperetin in humans after ingestion of orange juice and grapefruit juice. J Nutr 200 1; 13 1(2):235-41.

16. Brand W, Shao J, Hoek-van den Hil EF, et al. Stereoselective conjugation, transport and bioactivity of s- and R-hesperetin enantiomers in vitro. J Agric Food Chem 2010;58(10):61 19-25.

17. Grundy SM, Cleeman II, Daniels SR, et al. Diagnosis and management of the metabolic syndrome: an American Heart Association/National Heart, Lung, and Blood Institute scientific statement. Curr Opin Cardiol 2006; 21(1): 1-6

18. De Boever P, Deplancke B, Verstraete W. Fermentation by gut microbiota cultured in a simulator of the human intestinal microbial ecosystem is improved by supplementing a soygerm powder. J Nutr 2000; 130(10):2599-606.

19. Possemiers S, Verthé K, Uyttendaele S, Verstraete W. PCR-DGGE-based quantification of stability of the microbial community in a simulator of the human intestinal microbial ecosystem. FEMS Microbiol Ecol 2004;49(3):495-507.

20. Bonora E, Kiechl S, Willeit J, et al. Prevalence of insulin resistance in metabolic disorders: the Bruneck Study. Diabetes 1998;47(10): 1643-9.

21. Katz A, Nambi SS, Mather K, et al. Quantitative insulin sensitivity check index: a simple, accurate method for assessing insulin sensitivity in humans. J Clin Endocrinol Metab 2000;85(7):2402-10.

22. Matsuda M, DeFronzo RA. Insulin sensitivity indices obtained from oral glucose tolerance testing: comparison with the euglycemic insulin clamp. Diabetes Care 1999;22(9): 1462-70. 
23. de Bock M, Derraik JG, Brennan CM, et al. Olive (Olea europaea L.) leaf polyphenols improve insulin sensitivity in middle-aged overweight men: a randomized, placebo-controlled, crossover trial. PLoS One 2013;8(3): e57622.

24. Williamson G. Possible effects of dietary polyphenols on sugar absorption and digestion. Mol Nutr Food Res 2013:57(1):48-57.

25. Johnston K, Sharp P, Clifford M, Morgan L. Dietary polyphenols decrease glucose uptake by human intestinal Caco-2 cells. FEBS Lett 2005;579(7): 1653-7.

26. Qin B, Nagasaki $M$, Ren $M$, et al. Cinnamon extract (traditional herb) potentiates in vivo insulin-regulated glucose utilization via enhancing insulin signaling in rats. Diabetes Res Clin Pract 2003;62(3): 139-48.

27. Kim EK, Kwon KB, Song MY, et al. Flavonoids protect against cytokine-induced pancreatic beta-cell damage through suppression of nuclear factor kappaB activation. Pancreas 2007;35(4):e 1-9.

28. Jung UJ, Lee MK, Park YB, Kang MA, Choi MS. Effect of citrus flavonoids on lipid metabolism and glucoseregulating enzyme mRNA levels in type-2 diabetic mice. Int J Biochem Cell Biol 2006;38(7): 1 134-45.

29. Rivera L, Morón R, Sánchez M, Zarzuelo A, Galisteo M. Quercetin ameliorates metabolic syndrome and improves the inflammatory status in obese Zucker rats. Obesity (Silver Spring) 2008; 16(9):208 1-7.

30. Cani PD, Neyrinck AM, Fava F, et al. Selective increases of bifidobacteria in gut microflora improve highfat-diet-induced diabetes in mice through a mechanism associated with endotoxaemia. Diabetologia 2007; 50( 1 1): 2374-83

31. Guinane CM, Cotter PD. Role of the gut microbiota in health and chronic gastrointestinal disease: understanding a hidden metabolic organ. Therap Adv Gastroenterol 2013;6(4):295-308.

32. den Besten G, van Eunen K, Groen AK, et al. The role of short-chain fatty acids in the interplay between diet, gut microbiota, and host energy metabolism. J Lipid Res 2013;54(9):2325-40.

33. Tolhurst $G$, Heffron H, Lam YS, et al. Short-chain fatty acids stimulate glucagon-like peptide- 1 secretion via the G-protein-coupled receptor FFAR2. Diabetes 2012;61(2):364-71.

34. Boden G, Shulman GJ. Free fatty acids in obesity and type 2 diabetes: defining their role in the development of insulin resistance and beta-cell dysfunction. Eur J Clin Invest 2002;32 Suppl 3: 14-23.

35. Hamer HM, Jonkers DM, Bast A, et al. Butyrate modulates oxidative stress in the colonic mucosa of healthy humans. Clin Nutr 2009;28(1):88-93.

36. Vrieze A, Van Nood E, Holleman F, et al. Transfer of intestinal microbiota from lean donors increases insulin sensitivity in individuals with metabolic syndrome. Gastroenterology 2012;143(4):913-6 e7.

37. Hamer HM, Jonkers D, Venema K, et al. Review article: the role of butyrate on colonic function. Aliment Pharmacol Ther 2008;27(2): 104-19.

38. Grassi D, Desideri G, Necozione S, et al. Blood pressure is reduced and insulin sensitivity increased in glucose-intolerant, hypertensive subjects after 15 days of consuming high-polyphenol dark chocolate. J Nutr 2008; 138(9): 1671-6.

39. Brown AL, Lane J, Coverly J, et al. Effects of dietary supplementation with the green tea polyphenol epigallocatechin-3-gallate on insulin resistance and associated metabolic risk factors: randomized controlled trial. Br J Nutr 2009; 101(6):886-94.

40. Mortensen $\mathrm{OH}$, Nielsen AR, Erikstrup C, et al. Calprotectin--a novel marker of obesity. PLoS One 2009:4(10):e7419.

41. Soldavini J, Kaunitz JD. Pathobiology and potential therapeutic value of intestinal short-chain fatty acids in gut inflammation and obesity. Dig Dis Sci 2013;58(10):2756-66.

42. Campeotto F, Suau A, Kapel N, et al. A fermented formula in pre-term infants: clinical tolerance, gut microbiota, down-regulation of faecal calprotectin and up-regulation of faecal secretory lgA. $\mathrm{Br} J$ Nutr 201 1; 105(12): 1843-51.

43. Vulevic J, Juric A, Tzortzis G, Gibson GR. A mixture of trans-galactooligosaccharides reduces markers of metabolic syndrome and modulates the fecal microbiota and immune function of overweight adults. J Nutr 2013; 143(3): 324-31.

44. Franssen R, Monajemi H, Stroes ES, Kastelein JJ. Obesity and dyslipidemia. Med Clin North Am 201 1:95(5):893-902.

45. Bok SH, Lee SH, Park YB, et al. Plasma and hepatic cholesterol and hepatic activities of 3-hydroxy-3-methylglutaryl-CoA reductase and acyl CoA: cholesterol transferase are lower in rats fed citrus peel extract or a mixture of citrus bioflavonoids. J Nutr 1999; 129(6): 1 182-5. 
46. Kim HK, Jeong TS, Lee MK, et al. Lipid-lowering efficacy of hesperetin metabolites in high-cholesterol fed rats. Clin Chim Acta 2003;327(1-2): 129-37.

47. Kurowska EM, Spence JD, Jordan J, et al. HDL-cholesterol-raising effect of orange juice in subjects with hypercholesterolemia. Am J Clin Nutr 2000;72(5): 1095-100.

48. Xu JW, Ikeda K, Yamori Y. Upregulation of endothelial nitric oxide synthase by cyanidin-3-glucoside, a typical anthocyanin pigment. Hypertension 2004;44(2):217-22.

49. Actis-Goretta L, Ottaviani II, Keen CL, Fraga CG. Inhibition of angiotensin converting enzyme (ACE) activity by flavan-3-ols and procyanidins. FEBS Lett 2003;555(3):597-600.

50. Monagas M, Khan N, Andres-Lacueva C, et al. Effect of cocoa powder on the modulation of inflammatory biomarkers in patients at high risk of cardiovascular disease. Am J Clin Nutr 2009;90(5): 1 144-50.

51. Macfarlane GT, et al. Microbiological aspects of production of short-chain fatty acids in the large bowel. Physiological and Clinical Aspects of Short-Chain Fatty Acids. 1999, Cambridge: Cambridge University Press. 


\section{Supplemental material}

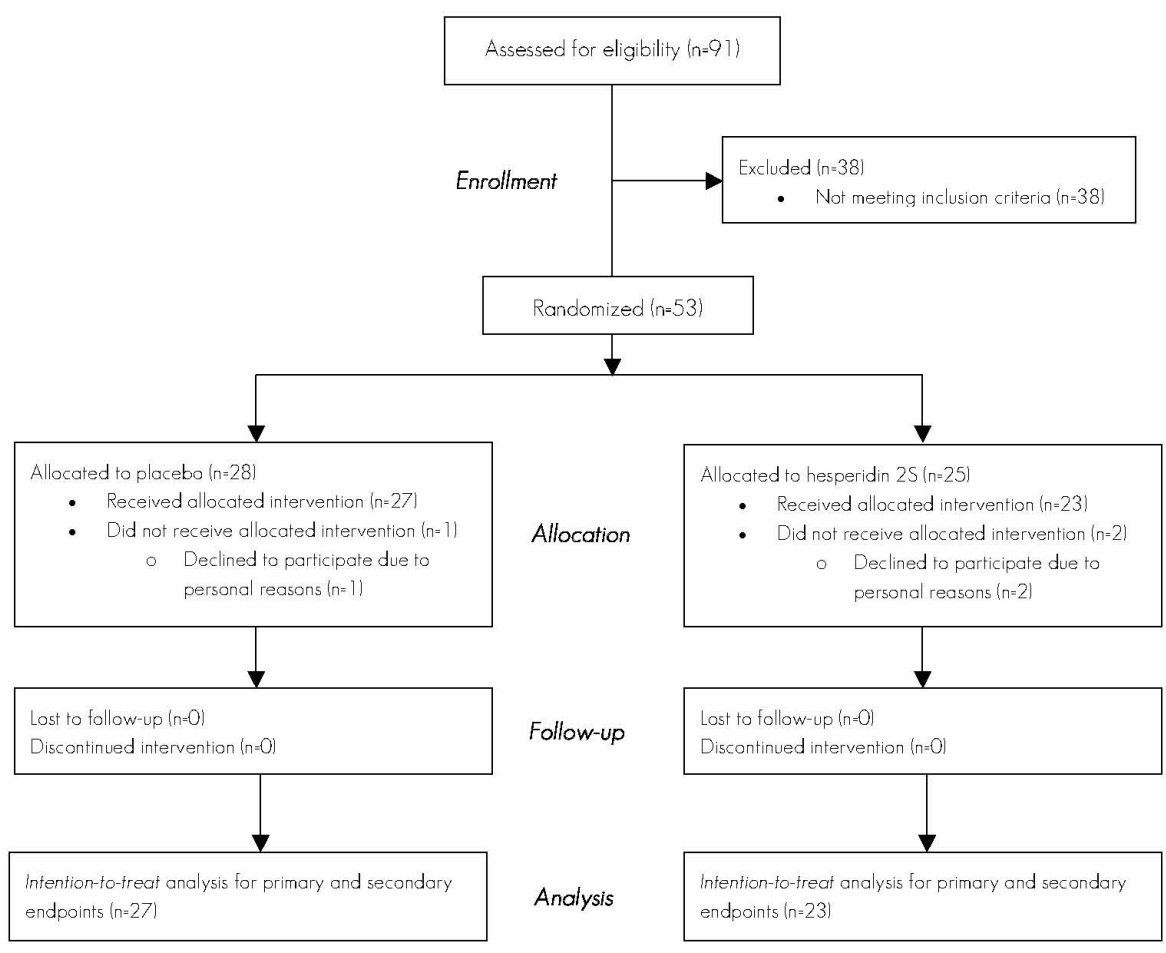

Figure S7.1 CONSORT flow diagram. 


$$
\text { 当 (0) (0) }
$$




\section{chapter 8}

Randomised controlled trial on the intestinal fate and effects of the carotenoid-producing Bacillus strain PDO1 on gastrointestinal and systemic parameters in overweight individuals

Salden BN, Stevens YR, Troost FJ, Possemiers S, Masclee AA 


\section{Abstract}

\section{Background}

Bacillus indicus PDO1 is a spore-forming bacterium, which naturally produces high levels of gastricstable carotenoids with superior bioavailability and antioxidant properties as compared to plant carotenoids. In combination with potential direct probiotic properties of PDO1 itself, the intestinal release of carotenoids may both have local effects in the intestine and systemic effects on cardiovascular or metabolic parameters. Until now no reports have been described confirming these properties in humans.

\section{Objective}

In this explorative study we aimed to confirm the intestinal fate of PDO 1 and the bioavailability of its carotenoids and to investigate the effect of 6 wks PDO 1 supplementation on both gastrointestinal (GI) and systemic parameters related to oxidative stress in overweight individuals.

\section{Design}

In this randomized, double-blind, placebo-controlled study, 68 overweight yet healthy individuals were randomly assigned to groups receiving PDO1 or placebo for 6 wks. On each test day, blood was sampled to measure bacterial carotenoids, antioxidant activity, oxidative stress biomarkers, adhesion molecules and metabolic markers. Fecal samples were obtained to assess the intestinal fate of PDOI and its effect on gut microbiota composition, microbial activity and calprotectin levels. Urine was collected to assess oxidative stress and $G$ permeability markers. Also blood pressure (BP) was measured, and questionnaires were filled out.

\section{Results}

PDO 1 was able to germinate efficiently $(73 \%$ vegetative cells in feces) and to release its carotenoids as shown by the detection of PDO 1 carotenoids in blood. In this explorative study no significant effects on intestinal barrier function nor microbiota composition nor intestinal or systemic effects could be confirmed. No adverse events were reported in relation to PDO1 intake, confirming that repeated intake of PDOI was well tolerated. Additionally, PDO1 intake was associated with a significant reduction in symptoms of indigestion.

\section{Conclusions}

This study provides the first evidence in humans that PDO1 is able to germinate and release bacterial carotenoids, which are absorbed and detected in human blood. No intestinal or systemic effects were observed but Gl symptoms improved significantly. These encouraging results warrant further largescale research in specific target populations to investigate the specific bioactivities of PDOl at intestinal and systemic level. 


\section{Introduction}

The incidence of cardiovascular diseases (CVD) varies worldwide, but is notably lower in countries where a 'Mediterranean diet' predominates. This diet contains relatively large amounts of fruit, vegetables and olive oil.' Carotenoids, the pigments responsible for the yellow to red color of some fruits and vegetables, have been indicated as one of the beneficial components of the Mediterranean Diet. ${ }^{2}$ Associations between high carotenoid intake and protection against oxidative stress, inflammation, endothelial dysfunction, hypertension and atherosclerotic progression have been demonstrated. ${ }^{3.4}$ The protective effects of carotenoids are related, at least in part, to their potent antioxidant capacity, which has been well demonstrated in in vitro assays. ${ }^{5}$ However, the relevance of their antioxidant properties in vivo is still debated. It is likely that other biological effects of carotenoids also contribute to their health protective effects. ${ }^{6}$

Carotenoids are lipophilic antioxidants, synthesized mainly in plants. Humans are not able to synthesize carotenoids and therefore are dependent on dietary sources. The majority of commercially available natural carotenoids are derived from plant-based materials. However, the quality and consistency of these carotenoids varies considerably. Interestingly, carotenoid-like molecules are also synthesized in nature by a number of bacterial species. In this respect, carotenoids produced by a variety of spore-forming bacillus strains are of specific interest. ${ }^{7.8}$ In vitro studies have shown that these bacterial carotenoids are stable in the stomach, are equally or even more bioaccessible and bioavailable than dietary carotenoids currently on the market, and exert a tenfold higher antioxidant activity compared to lycopene, one of the most potent antioxidants known. Furthermore, the carotenoid-producing bacillus strain itself may also possess probiotic properties. ${ }^{9}$

Previous in vitro and animal studies have provided evidence that a specific Bacillus indicus strain, named PDO 1, has excellent intestinal survival and can germinate and replicate under intestinal conditions (unpublished data). Furthermore, its carotenoids are released in the intestine and they were shown to have a high bioavailability and a promising biological activity profile with strong antioxidant properties and effects both on CVD risk markers and on the intestinal environment (unpublished data). As such effects have not yet been confirmed in humans, the present human intervention study was undertaken to investigate whether PDO 1 is able to survive transit through the gastrointestinal (GI) tract and thereby is able to release systemically absorbable carotenoids in an in vivo setting. Additionally, the $\mathrm{Gl}$ and systemic effects of the bacillus strain and its bacterial carotenoids were examined. We specifically chose to study a healthy, yet overweight-obese population, as this condition has previously been associated with higher risk of a wide range of $G$ l and systemic metabolic alterations including gut microbiota, ${ }^{10}$ intestinal barrier, ${ }^{11} \mathrm{Gl}$ and systemic lowgrade inflammation, ${ }^{12,13}$ endothelial function, ${ }^{14}$ hypertension ${ }^{15}$ and glucose and lipid homeostasis. ${ }^{16,17}$ We hypothesized that PDO1 is able to survive transit through the human Gl 
tract and that it is able to release bacterial carotenoids that can be absorbed and detected in human plasma. Furthermore, we hypothesized that 6 weeks daily PDO 1 supplementation beneficially affects 1) Gl microbiota, barrier and metabolic function and 2) systemic antioxidant activity and metabolic function and 3) is well-tolerated in overweight individuals. Primary aim of our study was to investigate the efficacy of 6 wks daily supplementation of PDOl on oxidative stress, as measured by $24 \mathrm{~h}$ urinary excretion of $\mathrm{F}_{2}$-isoprostanes, in healthy overweight individuals. Secondary, we aimed to assess the plasma bioavailability of the bacterial carotenoids during 6 wks daily PDO 1 supplementation, and to investigate: 1) Gl effects, by assessing gut microbiota composition, fecal short-chain fatty acids (SCFA) concentrations, Gl permeability, fecal calprotectin, digestive tolerance and 2) systemic effects, by measuring total antioxidant capacity, plasma malondialdehyde (MDA), plasma adhesion molecules, fasting blood glucose, insulin and lipids markers and blood pressure.

\section{Subjects and methods}

This study was approved by the Medical Ethics Committee of the Maastricht University Medical Center + (MUMC+) and conducted in full accordance with the principles of the Declaration of Helsinki of 1975 as amended in 2013 and with the Dutch Regulations on Medical Research involving Human Subjects (WMO, 1998). The study was performed at the MUMC+ from August 2015 to December 2015. All participants gave written informed consent before participation. The trial has been registered in the Clinical Trials register (NCTO2622425).

\section{Subjects}

Healthy, overweight or obese volunteers aged 18-70 years with a BMI between $25-35 \mathrm{~kg} / \mathrm{m}^{2}$ were recruited by advertisement. Exclusion criteria were: any medical condition that might interfere with the study and might jeopardize the health status of the participant; smoking; high intake of fruits and vegetables (> $75^{\text {th }}$ percentile of dietary intake of fruits and vegetables in general Dutch population); abuse of alcohol (> 20 alcoholic units / $w k$ ) and drugs; no consistently stable body weight for at least 3 months ( $\pm 3 \mathrm{~kg}$ ); plans to lose weight or following hypocaloric diet during study period; use of medication / vitamin-, mineral- or antioxidant supplements; consumption of pro-, pre- or synbiotics during study period and in 30 days prior to start of the study; use of antibiotics in the 90 days prior to start of the study; pregnancy and lactation; history of any side effects towards the intake of pro-, pre-, synbiotic supplements or carotenoids; failure to comply with prohibited intake of carotenoid-rich food products. During the study, the subjects consumed their habitual diet. At 
the time of inclusion, all subjects were informed about the prohibited carotenoid-rich food products, pro-, pre- and synbiotics.

\section{Design and intervention}

This study was designed as a randomized, placebo-controlled, double-blind, parallel-group study. Each subject underwent three test days. Participants were randomly assigned in a double-blind fashion to one of the two intervention arms: PDO1 or placebo (maltodextrin). An independent and blinded person generated the randomization list, using a computerized procedure. All participants and investigators remained blind to treatment until all analyses were completed. Participants were instructed to abstain from strenuous physical exercise, consumption of alcohol and carotenoid-rich food products on the day prior to each test day. Assessments were performed in a quiet, temperature-controlled $\left(20-24^{\circ} \mathrm{C}\right.$ ) room. Subjects handed in a fecal sample on the first test day. Then, anthropometric measurements (height, body weight, waist-to-hip circumference) and BP measurements were performed. Subsequently, venous blood samples were collected from an antecubital vein in the forearm. Then, subjects ingested a multi-sugar drink and collected full urine output for $24 \mathrm{~h}$ for measurement. Finally, the subject completed a questionnaire to assess the presence of $\mathrm{Gl}$ symptoms, stool frequency and stool consistency. After completion of the baseline measurements, participants received the study product for the following 6 wks. After 3 wks of daily supplementation, the second test day was organized. Measurements were identical to the baseline measurements performed during the first test day, with the exception of the multi-sugar drink with $24 \mathrm{~h}$ urine collection, which was not performed after 3 wks of intervention. After 6 wks of daily administration of the study product, the third test day was organized, which was identical to the first test day. Furthermore, a 3-day food record was completed prior to start of the study (during screening) and in the last week of the intervention period, to assess whether the participants maintained their dietary habits throughout the study period. To assess compliance, participants were asked to collect the empty sachets and to return these at the last visit.

\section{PDO1 and placebo}

PDO1 (ProDigest, Ghent, Belgium) is a food-grade bacterial formulation containing carotenoid-producing Bacillus indicus spores, which were grown and lyophilized on a maltodextrin (Pineflow; Matsutani Chemical Industry, Hyogo, Japan) carrier by Biopolis (Paterna, Valencia, Spain). The study products were provided as a powder in sachets. The PDO1 group received per day one sachet containing each $5^{*} 10^{9} \mathrm{CFU}$ PDO 1 with maltodextrin $(3 \mathrm{~g}$ ) as carrier material. The PDO1 carotenoid content of the clinical batch was quantified as $2.81 \mu \mathrm{g}$ per $10^{9} \mathrm{CFU}$, thus participants received in total $14.05 \mu \mathrm{g}$ PDO 1 
carotenoids per day. The placebo group received one sachet containing $3 \mathrm{~g}$ maltodextrin per day. Subjects were asked to stir the content of one sachet in $150 \mathrm{ml}$ whole fat milk and ingest the solution each morning, just before consuming breakfast, for 6 wks.

\section{Fecal PD01 quantification}

\section{Plate counting}

Prior to analysis, fecal samples were thawed for 20 minutes and homogenized in sterile distilled water. Homogenized fecal samples were serially diluted in PBS buffer. Part of the dilutions was pasteurized $\left(30 \mathrm{~min}\right.$ at $\left.65^{\circ} \mathrm{C}\right)$ to discriminate spores and viable cells. Both pasteurized and non-pasteurized samples were plated on agar plates, and incubated overnight at $37^{\circ} \mathrm{C}$. Yellow to orange colonies were counted as these represent PDO 1.

\section{Blood sample pretreatment}

\section{Plasma and serum isolation}

After blood collection, plasma was directly isolated by centrifugation ( $10 \mathrm{~min}, 1400 \mathrm{~g}, 4^{\circ} \mathrm{C}$ ) and stored at $-80^{\circ} \mathrm{C}$. For serum isolation, blood samples were first kept at room temperature for 30 minutes. Then, serum was isolated by centrifugation $\left(10 \mathrm{~min}, 1400 \mathrm{~g}, 21^{\circ} \mathrm{C}\right.$ ) and stored at $-80^{\circ} \mathrm{C}$.

\section{Bioavailability bacterial carotenoids}

After blood collection, blood samples were kept in an ice-water bath and light exposure was avoided. Plasma was isolated by centrifugation (10 min, $1400 \mathrm{~g}, 4^{\circ} \mathrm{C}$ ) within $2 \mathrm{~h}$ following collection and stored at $-80^{\circ} \mathrm{C}$.

\section{Bioavailability of bacterial carotenoids in plasma}

\section{Carotenoid extraction}

In a glass tube, $1 \mathrm{ml}$ of methanol containing internal standard (echinenone at $0.5 \mathrm{mg} / \mathrm{l}$ ) was added to $1 \mathrm{ml}$ of plasma sample. The mixture was vortexed for 10 minutes and $1 \mathrm{ml}$ of dichloromethane was added. After 10 additional minutes of vortexing, the mixture was centrifuged for 10 minutes at $10^{\circ} \mathrm{C}$ at $800 \mathrm{~g}$. The organic phase was collected and evaporated under argon gas. 


\section{Lipolysis of carotenoid esters}

The dry extract was dissolved in $500 \mu$ of $0.1 \mathrm{M}$ phosphate buffer at $\mathrm{pH} 7.5$ containing $30 \mathrm{mg} / \mathrm{ml}$ of bile salts. $250 \mu \mathrm{l}$ of lipase from Candida sp. and $250 \mu$ l of lipase from Thermomyces lanuginosis were added, and the solution was incubated for 1 hour at $37^{\circ} \mathrm{C}$ while stirring. After incubation, $500 \mu \mathrm{l}$ of methanol was added, the mixture was vortexed for 10 minutes, and $2 \mathrm{ml}$ of methyl tert-butyl ether (MTBE) was added. After 10 additional minutes of vortexing, the mixture was centrifuged for 10 minutes at $10^{\circ} \mathrm{C}$ at $800 \mathrm{~g}$. The organic phase was collected and evaporated under argon gas.

\section{HPLC analysis}

After evaporation to dryness, all extracts were dissolved in $200 \mu$ of methanol:MTBE (1:1, v:v) and carotenoids were quantified by reverse-phase HPLC using an HP 1100 Agilent system equipped with a YMC C30 column as described by Gleize et al. ${ }^{18}$ The nonesterified carotenoids were identified based on retention time and UV-Visible spectra (Figure 8.1).

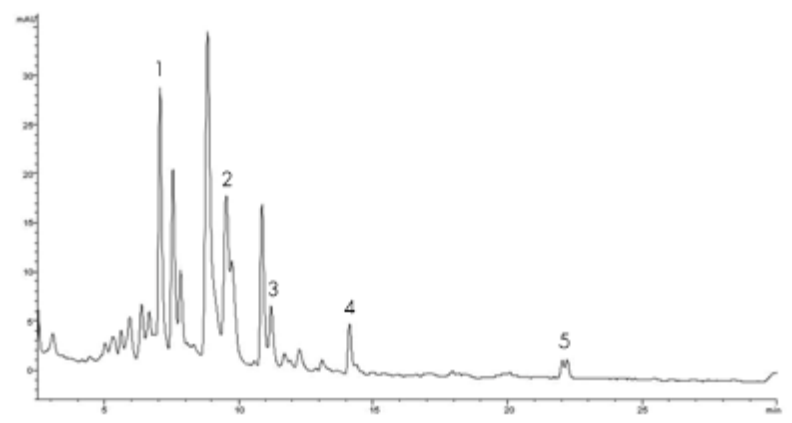

Figure 8.1 HPLC-UV VIS chromatogram of a lipophilic human plasma extract spiked with PDO1 carotenoids to validate the method (1: Lutein, 2: Methyl-glycosyl-apo-8'-lycopenoate (non-esterified form of orange carotenoid produced by PDO1 strain), 3: Glycosyl-apo-8'-lycopene (non-esterified form of yellow carotenoid produced by PDO 1 strain), 4: $\beta$-Carotene, 5: Lycopene).

\section{Fecal microbiota composition and microbial activity}

\section{DNA extraction}

Total genomic DNA suitable for PCR analysis was extracted from frozen human fecal samples using the OIAamp ${ }^{\circledast}$ DNA Stool Mini Kit (OIAGEN) following manufacturer's 
instructions. The PCR products were purified, a metagenomics library prepared, sequenced and analyzed, as previously described by Hevia et al. ${ }^{19}$

\section{Gut microbiota composition}

Partial 16S rRNA gene sequences (V3 and V4 region) were amplified and sequenced as previously reported by Milani et al. ${ }^{20}$ The obtained individual sequence reads were filtered to remove low quality and polyclonal sequences, trimmed, and exported as fastq files. These fasta files were processed using a Ql|ME-based custom script. ${ }^{21}$ Quality control retained reads with a length between 140 and 400 bp, mean sequence quality scores > 20, and allowed truncation at the first base if a low- quality moving $10 \mathrm{bp}$ window was found. Presence of homopolymers $>7 \mathrm{bp}$, and sequences with mismatched primers were omitted. In order to calculate downstream diversity measures (alpha and beta diversity indices, Unifrac analysis), 165 rRNA Operational Taxonomic Units (OTUs) were defined at $\geq 97 \%$ sequence homology using uclust. ${ }^{22}$ All reads were classified to the lowest possible taxonomic rank using QIIME and a reference dataset from the SILVA database. ${ }^{23}$ Similarities between samples were calculated by unweighted uniFrac. ${ }^{24}$ The range of similarities was calculated between 0 and 1 .

\section{Fecal pH, SCFA, lactate and B-glucuronidase activity}

Prior to analysis, fecal samples were thawed for $20 \mathrm{~min}$ and homogenized in sterile distilled water. Stool pH was determined for each sample upon tenfold dilution in sterile distilled water. pH was measured with a standard pH meter (Senseline F40; ProSense, Oosterhout, The Netherlands) with a conventional pH electrode (OP2 111 T; ProSense).

SCFA concentrations were determined as described previously by $V$ an de Wiele et al. ${ }^{25}$ Homogenized stool samples were centrifuged at $7600 \mathrm{~g}$ for 5 minutes. Cell free supernatant $(50 \mu \mathrm{l})$ was used for lactate measurement using a commercially available enzymatic assay kit (R-Biopharm, Darmstadt, Germany). Furthermore, cell free supernatant was diluted 1:1 (v:v) in sterile distilled water and transferred (50 $\mu$ ) into a 96-well plate containing $50 \mu \mathrm{l} /$ well of a $5 \mathrm{mM}$ solution of 4-nitrophenyl $\beta$-D-glucuronide (Sigma-Aldrich, St-Louis, Missouri, USA). After 30 minutes of incubation at $37^{\circ} \mathrm{C}$, the release of $\mathrm{p}$-nitrophenol was quantified based on the absorbance at $405 \mathrm{~nm}$ using a Synergy HT multiplate reader (BioTek Instruments, Winooski, Vermont, USA). The results were expressed in $\mu$ mol p-nitrophenol released per minute and per gram feces.

\section{Metabolomic analysis on fecal samples}

Metabolomic analysis was applied as previously described in detail by Vanden Bussche et al. ${ }^{26}$ 


\section{Gastrointestinal permeability}

Gastrointestinal permeability was assessed by using a validated multi-sugar test, measuring the $24 \mathrm{~h}$ urinary excretion of ingested sugar probes reflecting permeability of 4 segments of the $\mathrm{Gl}$ tract. The method of measurement has been described previously by Mujagic et al.. ${ }^{27}$ Urinary sugar probes were determined by HPLC-MS as previously described. ${ }^{28}$

\section{Fecal calprotectin}

Fecal calprotectin concentration levels were determined using an ELISA for human calprotectin (Hycult Biotechnology, Uden, Netherlands), according to the manufacturer's protocol.

\section{Antioxidant activity and biomarkers of oxidative stress}

\section{Total antioxidant capacity (TAC)}

Plasma TAC was measured by a commercially available quantitative colorimetric assay kit, according to the manufacturer's instructions (BioAssay Systems, Hayward, USA). Briefly, $\mathrm{Cu}^{2+}$ was reduced by the available antioxidants in the sample to $\mathrm{Cu}_{+}$. The resulting $\mathrm{Cu}^{+}$ formed a colored complex with a dye reagent, which was quantified colorimetrically (Bio-Tek, Synergy HT, Winooski, USA). This is proportional to the TAC in the sample. The total antioxidant capacity was expressed in $\mathrm{m} M$ Trolox equivalents.

\section{Urinary F2-isoprostanes}

Urinary $F_{2}$-isoprostane concentration was measured by a commercially available enzyme immune assay (EIA) kit, according to the manufacturer's instructions (Cayman Chemical Co., Ann Arbor, MI). Briefly, $500 \mu$ urine was acidified to $\mathrm{pH} 4$ and samples were applied to prewashed C-18 SPE cartridges (Cayman Chemical Co, Ann Arbor, MI), after which the eluate was added. The ethyl acetate/methanol elvate from the SPE cartridges was subsequently dried using vacuum centrifugation and reconstituted in EIA buffer. The samples were assayed in duplicate with standards (0-3000 pg/ml of 8-iso-PGF2a), and blanks. After an incubation period of $1 \mathrm{~h}$ the plate was washed and pNPP reagent was added. The resultant color reaction was read after approximately $1 \mathrm{~h}$ using a Bio-Rad plate reader at $420 \mathrm{~nm}$, the color development being inversely proportional to the concentration of 8-isoPGF2a measured. To optimize the assay, it was necessary to use ultrapure water for all aqueous reagents and to manually wash the plates. 


\section{Malondialdehyde (MDA)}

Plasma MDA was quantified by a commercially available microplate assay kit, according to the manufacturer's protocol (Cohesion Biosciences Limited, London, United Kingdom). Briefly, the MDA in the sample reacted with Thiobarbituric Acid (TBA) to generate the MDA-TBA adduct. The MDA-TBA adduct was quantified colorimetrically (Bio-Tek, Synergy HT, Winooski, USA).

\section{Adhesion molecules}

Circulating soluble vascular cell adhesion molecule-1 (sVCAM-1), soluble intercellular adhesion molecule-1 (s|CAM-1), soluble E-selectin (sE-selectin) and soluble P-selectin (sP-selectin) were determined by commercially available ELISA and standards, according to the manufacturer's protocol (Bender MedSystems GmbH, Vienna, Austria).

\section{Blood glucose, insulin and lipids}

Serum concentrations of glucose, total cholesterol, low-density lipoprotein (LDL)- and highdensity lipoprotein (HDL)-cholesterol and triglycerides were measured by spectrophotometry (cobas 6000 analyzer series, Roche Diagnostics, Indianapolis, USA). Plasma insulin was determined using a luminescence-enhanced immune-enzymatic assay (Immulite 2000 immunoassay system, Siemens Healthcare, Erlangen, Germany). Insulin resistance (IR) was estimated by the homeostasis model assessment of insulin resistance (HOMA-IR) index. It was calculated as the product of fasting plasma glucose (mmol/L) and insulin (mU/l) concentrations divided by 22.5. Insulin sensitivity (IS) was assessed using the quantitative insulin sensitivity index (OUICKI), calculated as: 1 / (log insulin $\mathrm{O}$ h + log glucose $\mathrm{Oh}$.

\section{Blood pressure and heart rate measurements}

BP was monitored using a semi-continuous BP monitoring device 1 Omron, Hoofddorp, The Netherlands) at the upper right arm. At each occasion, after 30 minutes of rest in the supine position, 4 consecutive BP measurements were performed. The first measurement was discarded and the remaining 3 measurements were averaged.

\section{Digestive tolerance}

The occurrence of $\mathrm{Gl}$ symptoms was assessed using the validated gastrointestinal symptom rating scale (GSRS), consisting of 15 items combined into five symptom clusters describing reflux, abdominal pain, indigestion, diarrhea, and constipation. ${ }^{29}$ In the seven-point graded 
Likert-type GSRS, a score of 1 represents absence of troublesome symptoms and a score of 7 represents very troublesome symptoms. Defecation frequency and stool consistency were assessed using the Bristol Stool Form Scale Chart. ${ }^{30}$

\section{Statistical analysis}

The primary outcome of the study was the effect of 6 wks PDO 1 supplementation on $24 \mathrm{~h}$ urinary excretion of $F_{2}$-isoprostanes, compared to placebo. Sample size was determined for the primary outcome of the study, using a significance level alpha $=0.05$ and a power of $90 \%$. Based on previous research," we calculated that a sample size of 60 subjects would be required to detect a difference in $24 \mathrm{~h}$ urinary $F_{2}$-isoprostanes of $104 \pm 85 \mathrm{pg} / \mathrm{ml}$ (mean \pm SD). Baseline characteristics are presented as mean (SD) for numerical variables and number $(\%)$ for categorical variables. Differences in gut microbiota composition, microbial activity, Gl permeability, GI inflammation, antioxidant activity, oxidative stress, adhesion molecules, metabolic markers, BP, and questionnaires between intervention groups (PDO1 or placebo) were assessed using linear mixed models with group (placebo and PDO 1), time (1, 3 and 6 wks for 3 and 6 wks PDO 1 effect) and group*time as fixed factors, where an unstructured covariance structure was used for repeated measures. The linear mixed model accounts for the correlation between repeated measures and missing data, where a likelihood approach was used assuming data missing at random. The estimated means and SEM obtained from this model were presented for each group and each time point. In case of asymmetrical distribution of data, medians with interquartile ranges were displayed. The Friedman test with post-hoc Wilcoxon Signed Rank test was applied to compare the presence of PDOI in feces and bacterial carotenoids in plasma between baseline samples and samples obtained after 3 and 6 wks PDO1 supplementation. The Wilcoxon Singed Rank test was used to compare the fecal microbiota composition at genus level between baseline samples and samples obtained after 6 wks supplementation in a treatment group. These statistical analyses were performed using IBM SPSS Statistics for Windows (version 21.0, Armonk, NY, USA).

For the metabolomics analysis, chemometric data analysis was applied. First, extensive data pre-processing was conducted on the obtained full scan HRMS data files with Sieve ${ }^{\text {TM }} 2.2$ software (Thermo Fischer Scientific). Next, normalization of the dataset was performed to correct for instrumental drift. Next, a logarithmic transformation and a Pareto scaling $(1 / \sqrt{ } S D$, where SD is the standard deviation) were performed for inducing normality and standardizing the range of independent X-variables, respectively. Finally, multivariate regression techniques (Simca 13.5.0, Umetrics, Sweden) were used to differentiate between the obtained fingerprints. Principal component analysis (PCA) was used to reveal outliers, groups and trends, whereas (orthogonal) partial least square-discriminant analysis (IO)PLSDA) was used to construct a prediction model that could predict the treatment from the 
fecal metabolic fingerprint to explore possible suppressed metabolic effects. For all analyses, two-sided $P$ values $\leq 0.05$ were considered statistically significant.

\section{Results}

\section{Subjects}

In total 67 healthy volunteers were enrolled in the study, of which 62 completed the entire study protocol. One participant never started participation for a personal reason. Four participants dropped out during the intervention period; one due to the need for antibiotic treatment, another for unspecified, private reasons, while two participants experienced mild Gl complaints. These complaints were present already at the start of the study and not associated to PDO1 intake. From these participants only baseline characteristics were available. Baseline characteristics are presented in Table 8.1.

Table 8.1 Baseline characteristics of study participants.

\begin{tabular}{lccc}
\hline & $\begin{array}{c}\text { Total population } \\
(n=67)\end{array}$ & $\begin{array}{c}\text { Placebo } \\
(n=34)\end{array}$ & $\begin{array}{c}\text { PD01 } \\
(n=33)\end{array}$ \\
\hline Age, $y r$ & $53 \pm 13^{2}$ & $50 \pm 15$ & $56 \pm 10$ \\
Sex, $/ \mathrm{F}$ & $29 / 38$ & $12 / 22$ & $17 / 16$ \\
WHR & $0.91 \pm 0.08$ & $0.90 \pm 0.08$ & $0.93 \pm 0.08$ \\
BMl, $\mathrm{kg} / \mathrm{m}^{2}$ & $29.9 \pm 3.4$ & $30.3 \pm 3.6$ & $29.5 \pm 3.2$ \\
\hline
\end{tabular}

'BMI, body mass index. WHR, waist-to-hip-ratio. ${ }^{2}$ Mean \pm SD (all such values).

\section{Fecal PDO 1 quantification}

PDO1 was not detected in any of the fecal samples of subjects receiving placebo. In the PDO1 group, PDO1 was not detected in any of the fecal samples of any of the subjects at baseline, but was present in fecal samples of all subjects after 3 wks of supplementation and in 27 of the 29 subjects after 6 wks of supplementation. There was a statistically significant difference in PDO 1 total cells $\left(\chi^{2}(2)=39.38, P=0.000\right)$ and spores $\left(\chi^{2}(2)=42.74\right.$, $P=0.000$ ) in feces after PD0 1 supplementation. Post hoc analysis with Wilcoxon signed-rank tests was conducted with a Bonferroni correction applied, resulting in a significance level set at $P<0.017$. As shown in Table 8.2, significantly higher amounts of PDO 1 total cells were found in feces both after 3 wks and 6 wks supplementation, compared to baseline lall $P<0.0011$. No significant differences could be observed between 3 and 6 wks supplementation both for total cells and spores (both $P \geq 0.039$ ), pointing to a steady state after 3 wks of PDO 1 supplementation. The total amount of viable PDO 1 cells in human feces after 6 wks of PDO 1 supplementation was quantified as $2.26^{*} 10^{7} \mathrm{CFU} / \mathrm{g}$, of which $3.43^{*} 10^{6}$ 
CFU/g are PDO1 spores. While administered as spores, PDO1 was therefore mainly present in feces as vegetative cells $\left(1.99 * 10^{7} \mathrm{CFU} / \mathrm{g} ; 73 \%\right.$ of total counts), indicating effective germination under intestinal conditions.

Table $8.2 \quad$ PDO total cells and spores (CFU/g) in fecal samples at baseline, after 3 wks and after 6 wks supplementation with PDO1 ( $n=29)^{\text {? }}$

\begin{tabular}{|c|c|c|c|}
\hline & Baseline & 3 wks & 6 wks \\
\hline Total cells ${ }^{3,4}$ & $\mathrm{O}[0 ; \mathrm{O}]^{2}$ & $2.50 \mathrm{E7}$ [1.1 1E7; 3.98E7] & 2.26E7 [1.06E7; 4.19E7] \\
\hline Spores 3.4 & $0[0 ; 0]$ & 5.77E6 [1.28E6; 1.56E7] & 3.43E6 [1.85E6; 1.06E7] \\
\hline Percentage of total & 0 & $30.2[11.1 ; 52.9]$ & $26.6[6.7 ; 39.5]$ \\
\hline Vegetative cells $s^{3,4}$ & $\mathrm{O}[0 ; \mathrm{O}]$ & 1.70E7 [4.20E6; 3.95E7] & 1.99E7 [5.65E6; 2.93E7] \\
\hline Percentage of total & 0 & $69.8[47.1 ; 88.9]$ & 73.4 [60.5; 93.3] \\
\hline
\end{tabular}

'Differences between baseline and 3 wks and 6 wks supplementation tested with Friedman test with post hoc Wilcoxon signed rank test. ${ }^{2}$ Medians [Q1; @3] (all such values). ${ }^{3}$ Significant difference between baseline vs. 3 wks intervention. ${ }^{4}$ Significant difference between baseline vs. 6 wks intervention.

\section{Bioavailability of bacterial carotenoids in plasma}

PDO1 carotenoids were detected in fasted plasma samples of all subjects after 3 wks and after 6 wks of PDOl supplementation. The plasma concentration of PDOl carotenoids significantly increased during the 6 wks of daily supplementation $10.000 \mu \mathrm{M}$ at baseline, $0.044 \mu \mathrm{M}$ at 3 wks, $0.076 \mu \mathrm{M}$ at 6 wks, respectively; all $P \leq 0.009$, Table 8.3). The presence of (bacterial) carotenoids in plasma samples of subjects of the placebo group was not assessed. PDO1 supplementation did not induce significant changes in the plasma content of lutein, $\beta$-carotene or lycopene during the study period (all $P \geq 0.121$, Table 8.3).

Table 8.3 Carotenoid concentrations $(\mu M)$ in fasted plasma at baseline and after 3 wks and 6 wks supplementation with PDO1 ( $n=29)$.

\begin{tabular}{lccc}
\hline & Baseline & 3 wks & 6 wks \\
\hline PDO 1 carotenoids & $0[0 ; .4 .5]^{2}$ & $0.044[0.002-0.167]^{1}$ & $0.076[0.019-0.233]$ \\
Lutein & $0.126[0.038-0.267]$ & $0.124[0.034-0.283]$ & $0.121[0.035-0.237]$ \\
$\beta$-Carotene & $0.279[0.029-0.887]$ & $0.316[0.036-0.786]$ & $0.317[0.031-0.786]$ \\
Lycopene & $0.181[0.018-0.461]$ & $0.182[0.026-0.445]$ & $0.177[0.018-0.392]$ \\
\hline
\end{tabular}

'Differences between baseline and 3 wks and 6 wks supplementation tested with Friedman test with post hoc Wilcoxon signed rank test. ${ }^{2}$ Medians [Q1; @3] (all such values). ${ }^{3}$ Significant difference between baseline vs. 3 wks intervention. ${ }^{4}$ Significant difference between baseline vs. 6 wks intervention. ${ }^{5}$ Significant difference between 3 wks and 6 wks intervention. 


\section{Fecal microbiota and metabolic activity}

\section{Relative abundance at phylum and genus level}

The dominant phyla across all baseline fecal samples were firmicutes (mean 80.3\%, SD 10.9\%) and bacteroidetes (mean 10.3\%, SD 10.0\%), followed by actinobacteria (mean $6.3 \%$, SD 5.4\%) and proteobacteria (mean 0.9\%, SD 3.6\%). The relative abundance of these 4 dominant phyla did not change significantly during 6 wks intake of PDO 1, compared to placebo (Figure 8.2). The obtained sequence reads were analyzed to obtain information on the bacterial composition of each sample at genus level. No significant changes in bacterial composition at genus level between the two treatments were observed during intervention. When analyzing the bacterial composition at genus level within the PDO1 group, we observed significant increases in the fecal abundance of Alloprevotella $(P=0.044)$, Blautio $(P=0.020)$, Butyrivibrio $(P=0.017)$, Dorea $(P=0.033)$, Flavonifractor $(P=0.037)$, and a species belonging to the ruminococcaceae family $(P=0.010)$ after 6 wks intervention, while in the placebo group the fecal abundance of none of these bacteria significantly altered (all $P \geq 0.163$ ). These effects are, however, no longer significant after any multiple testing correction (e.g. Bonferroni).

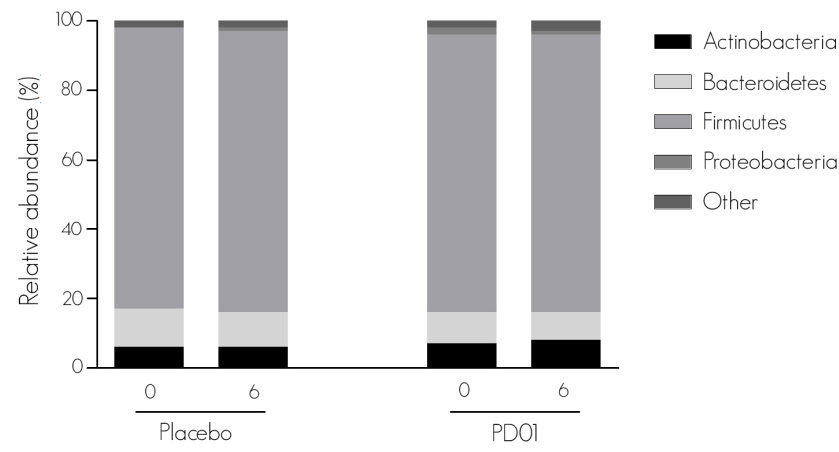

Figure 8.2 Relative abundance of the main phyla according the 16S-targeted Illumina data for placebo and PDO 1 at baseline (O) and after 6 wks supplementation (6). No significant differences in relative abundance between placebo and PDO1 were observed during study period, as tested with linear mixed model with correction for baseline values.

\section{Fecal samples alpha diversity}

Alpha diversity was calculated through the alpha_diversity.py script using different metrics (Chao, Shannon diversity index) to take into account the species evenness and richness in each sample (Figure 8.3A and Figure 8.3B). These alpha diversity plots show that the 
microbial diversity was very similar between the two interventions at baseline and after 6 wks of supplementation and, therefore, that intervention did not have major effects on the overall microbial diversity of the subjects.
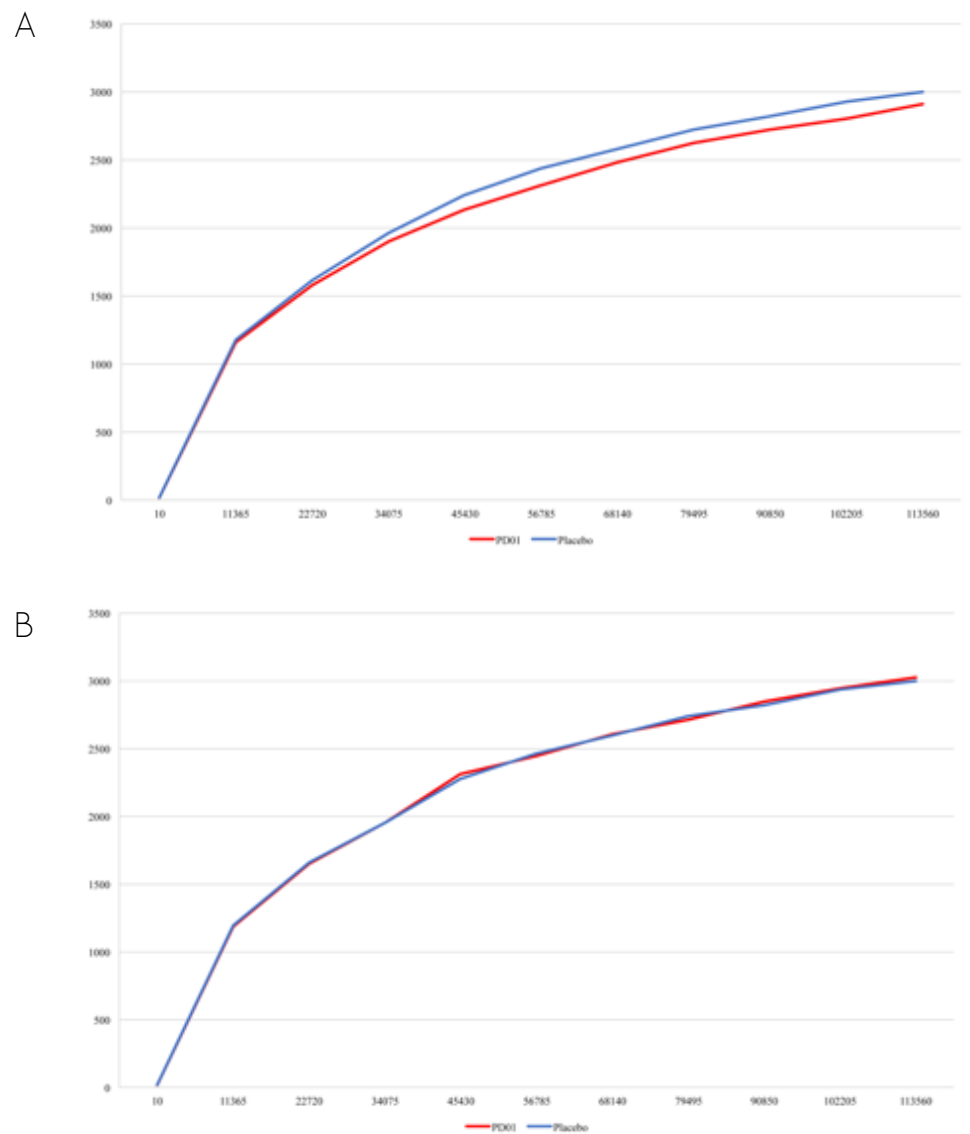

Figure 8.3 Alpha-diversity plots obtained using the Chao 1 index at baseline (A) and after 6 wks of treatment (B). (red: PDO 1; blue: placebo).

\section{Fecal samples beta diversity}

The beta diversity index was evaluated in order to analyze the microbial diversity between samples. As shown in Figure 8.4, the sequences from the various samples were not organized into clusters but were evenly distributed, indicating that the treatment did not have major effects on the overall microbial diversity of the human gut. 


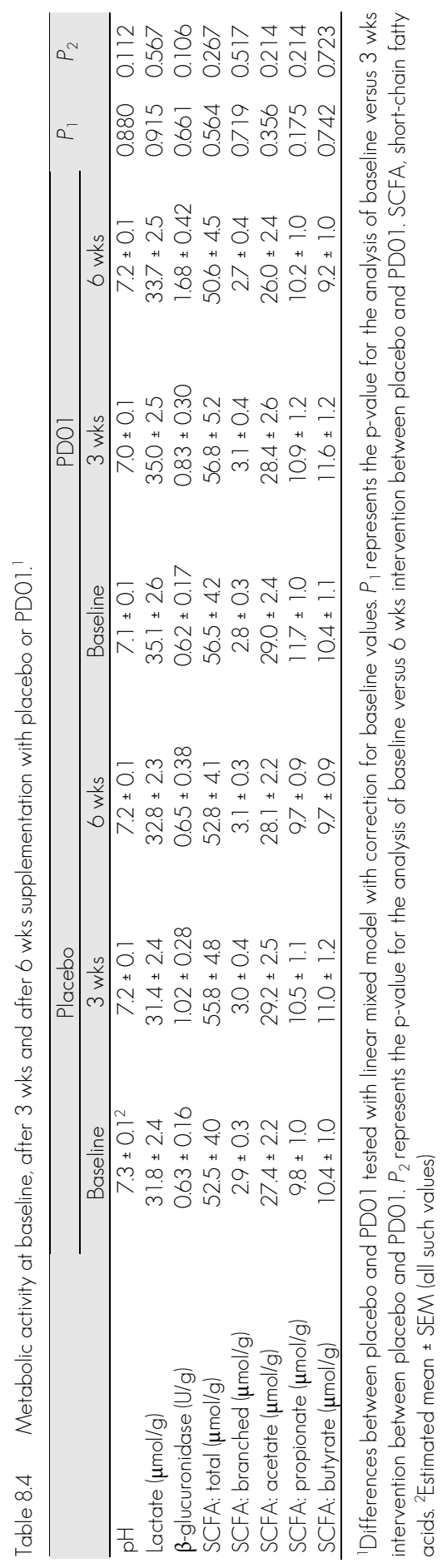



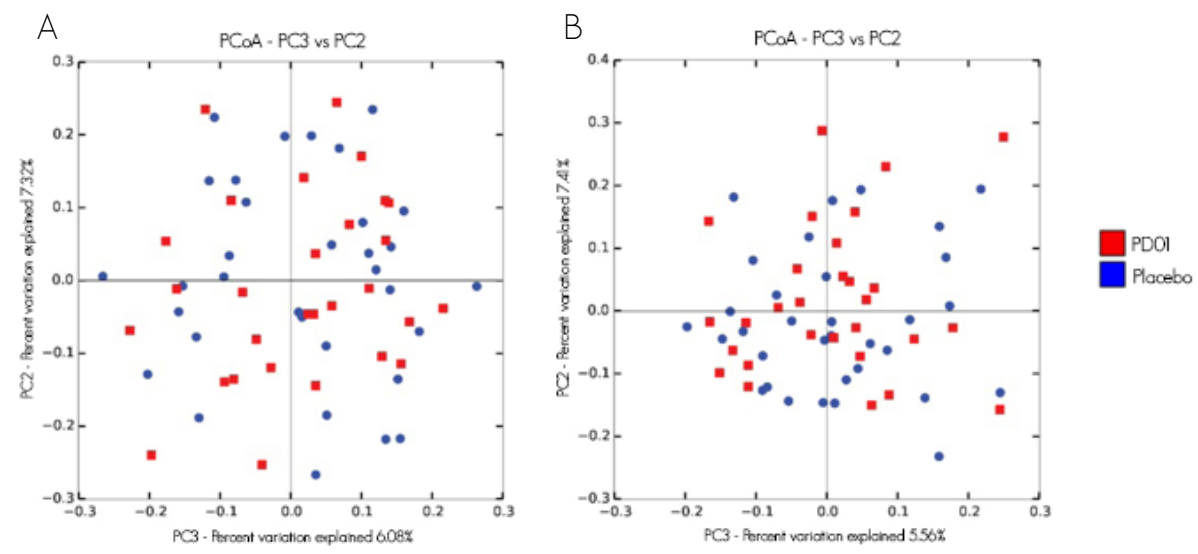

Figure 8.4 Example of beta-diversity plots comparing samples from participants receiving placebo (blue symbols) and PDO 1 (red symbols), collected at baseline (A) and after 6 wks supplementation (B).

\section{Fecal pH, SCFA, lactate and $\beta$-glucuronidase activity}

Results on gut metabolic activity are shown in Table 8.4. Stool pH and lactate did not significantly alter during PDO 1 supplementation, compared to placebo. Intake of PDO1 did not significantly change the total level of SCFA, branched SCFA and the levels of the individual SCFA, compared to placebo. Neither were differences in $\beta$-glucuronidase activity after 3 wks and after 6 wks of treatment between the two intervention groups observed.

\section{Metabolomics analysis of fecal samples}

Figure 8.5 represents the PCA score plot for samples obtained at baseline and after 6 wks PDO1 supplementation. No effect of intervention was observed. To investigate more in depth the metabolic effects induced by PDO1, supervised OPLS-DA models were constructed per ionization mode. For the datasets, the OPLS-DA score plot (Figure 8.6) also showed no significant effect of PDOI intervention. Individuals tend to cluster as other variables (e.g. genetic variation, lifestyle) induced more pronounced metabolic changes than the type of intervention. To reduce the inter-individual effect, the datasets were categorized according to the combination of treatment and time of sampling, resulting in 6 categories. In this way, it was possible to compare the different time points within the PDOI treated individuals. No significant changes in metabolomics between interventions were observed throughout the study. The PCA score plots displayed a good clustering of all quality control samples, but no clear trend could be observed. 


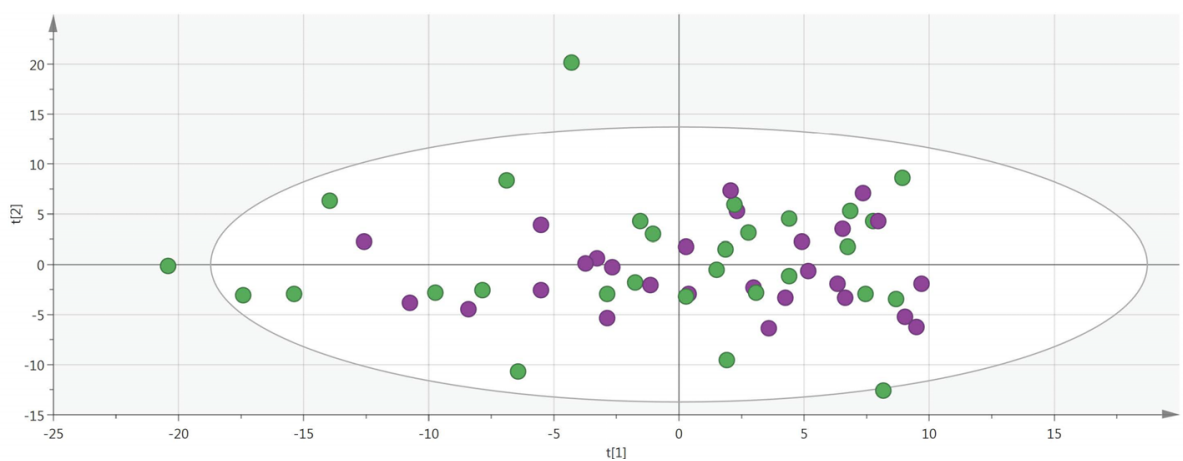

Figure 8.5 PCA score plot for the dataset categorized according to a combination of PDOI and time of sampling, where comparison is made between the human fecal metabolome at baseline (green) and after 6 wks of PDO 1 supplementation (purple).

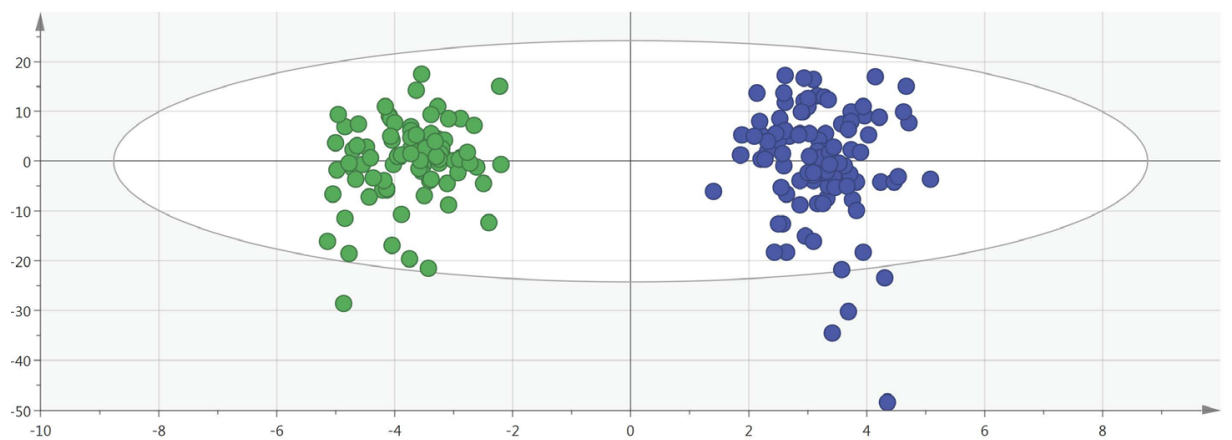

Figure 8.6 OPLS-DA score plot for the positively charged ions detected in the human feces by means of UHPLC-HRMS. (green: PDO1 treatment including test day 1, 2 and 3; blue: Placebo including test day 1, 2 and 3).

\section{Gastrointestinal permeability}

Table 8.5 presents the $G$ permeability data. No statistically significant changes in gastroduodenal, small intestinal or colonic and whole gut permeability were observed after 6 wks PDO1 supplementation compared to placebo. When analyzing the results in more detail it is apparent that a $37 \%$ decrease in gastroduodenal permeability was observed in response to PDO1 compared to a $5 \%$ decrease in response to placebo $(P=0.131)$. In addition, a 33\% decrease in colonic permeability was observed in the PDO1 group compared to no decrease in the placebo group $(P=0.104)$. 
Table 8.5 Permeability test: sugar excretion ( $\mu$ mol) and ratios of excreted sugars as measured in urine (in 0-2, 0-5, 5-24 and 0-24 h fraction). ${ }^{1}$

\begin{tabular}{|c|c|c|c|c|c|}
\hline & \multicolumn{2}{|c|}{ Placebo } & \multicolumn{2}{|c|}{ PDO1 } & \multirow[t]{2}{*}{$P$ value } \\
\hline & Baseline & 6 wks & Baseline & 6 wks & \\
\hline 0-5 h sucrose & $\begin{array}{c}5.290 \\
{[2.930 ; 11.625]^{2}}\end{array}$ & $\begin{array}{c}5.030 \\
{[3.460 ; 12.520]}\end{array}$ & $\begin{array}{c}6.975 \\
{[2.140 ; 19.248]}\end{array}$ & $\begin{array}{c}4.370 \\
{[2.540 ; 9.575]}\end{array}$ & 0.131 \\
\hline 0-5 h L/R ratio & $\begin{array}{c}0.030 \\
{[0.020 ; 0.040]}\end{array}$ & $\begin{array}{c}0.030 \\
{[0.020 ; 0.040]}\end{array}$ & $\begin{array}{c}0.035 \\
{[0.020 ; 0.050]}\end{array}$ & $\begin{array}{c}0.030 \\
{[0.020 ; 0.040]}\end{array}$ & 0.842 \\
\hline 5-24 h S/E ratio & $\begin{array}{c}0.010 \\
{[0.010 ; 0.020]}\end{array}$ & $\begin{array}{c}0.010 \\
{[0.010 ; 0.015]}\end{array}$ & $\begin{array}{c}0.015 \\
{[0.010 ; 0.020]}\end{array}$ & $\begin{array}{c}0.010 \\
{[0.010 ; 0.020]}\end{array}$ & 0.104 \\
\hline 0-24 h S/E ratio & $\begin{array}{c}0.010 \\
{[0.010 ; 0.020]}\end{array}$ & $\begin{array}{c}0.010 \\
{[0.010 ; 0.010]}\end{array}$ & $\begin{array}{c}0.010 \\
{[0.010 ; 0.020]}\end{array}$ & $\begin{array}{c}0.010 \\
{[0.010 ; 0.020]}\end{array}$ & 0.266 \\
\hline
\end{tabular}

'Differences between placebo and PDO1 tested with linear mixed model. L/R, lactulose/l-rhamnose. S/E, sucralose/erythritol. ${ }^{2}$ Median $[I Q R$, i.e. Q1; @3] (all such values).

\section{Fecal calprotectin}

Fecal calprotectin did not significantly change after 6 wks PDO1 supplementation, compared to placebo (placebo: $32.6 \mathrm{ng} / \mathrm{ml}$ to $36.2 \mathrm{ng} / \mathrm{ml}$; PDO 1: $46.6 \mathrm{ng} / \mathrm{ml}$ to $42.9 \mathrm{ng} / \mathrm{ml}$; $P=0.3411$.

\section{Antioxidant activity and biomarkers of oxidative stress}

Plasma TAC and MDA, and 24 h urinary excretion of $F_{2}$-isoprostanes were not significantly changed during 6 wks PDO1 supplementation, compared to placebo (Table 8.6).

Table 8.6 Antioxidant activity and biomarkers of oxidative stress at baseline and after 6 wks supplementation with placebo or PD01.'

\begin{tabular}{|c|c|c|c|c|c|}
\hline & \multicolumn{2}{|c|}{ Placebo } & \multicolumn{2}{|c|}{ PDO1 } & \multirow[t]{2}{*}{ Pvalue } \\
\hline & Baseline & $6 w k s$ & Baseline & 6 wks & \\
\hline Total antioxidant capacity $(\mu M)$ & $366 \pm 10^{2}$ & $351 \pm 9$ & $375 \pm 10$ & $363 \pm 9$ & 0.777 \\
\hline $\mathrm{F}_{2}$-isoprostanes $(\mathrm{pg} / \mathrm{mL})$ & $\begin{array}{c}169 \\
{[100 ; 242]^{3}}\end{array}$ & $\begin{array}{c}142 \\
{[82 ; 290]}\end{array}$ & $\begin{array}{c}171 \\
{[108 ; 312]}\end{array}$ & $\begin{array}{c}157 \\
\text { [99; 289] }\end{array}$ & 0.323 \\
\hline Malondialdehyde (nmol/mL) & $\begin{array}{c}0.21 \\
{[0.15 ; 0.28]}\end{array}$ & $\begin{array}{c}0.21 \\
{[0.15 ; 0.26]}\end{array}$ & $\begin{array}{c}0.21 \\
{[0.15 ; 0.26]}\end{array}$ & $\begin{array}{c}0.21 \\
{[0.15 ; 0.26]}\end{array}$ & 0.435 \\
\hline
\end{tabular}

'Differences between placebo and PDO1 tested with linear mixed model with correction for baseline values. ${ }^{2}$ Estimated mean \pm SEM (all such values). ${ }^{3}$ Median [IOR, i.e. O 1; @ 3 ] (all such values).

\section{Adhesion molecules}

Basal circulating adhesion molecules at baseline and after 6 wks intervention for both the placebo and the PDO1 groups are presented in Table 8.7. No significant differences in sVCAM, sICAM, sE-selectin and sP-selectin concentrations were observed between the two interventions. 


\section{Blood glucose, insulin and lipids}

Baseline total cholesterol, LDL- and HDL-cholesterol, triglycerides, glucose and insulin were all within normal blood value ranges. These parameters were not significantly altered by 3 wks or 6 wks supplementation with PDOl compared to placebo (Table 8.7). Also, no significant changes in insulin resistance and insulin sensitivity, expressed by the HOMA-IR and QUICKI indices, were observed between intervention groups (Table 8.7).

\section{Blood pressure and heart rate}

Baseline systolic BP, diastolic BP and HR were all within normal value ranges at baseline. No significantly changes regarding these parameters were observed between intervention groups both after 3 wks and 6 wks supplementation (data not shown).

\section{Digestive tolerance}

Participants receiving daily PDO1 scored lower on the GSRS subdimension for indigestion syndrome after 3 wks supplementation $(P=0.061$, Figure 8.7$)$, becoming significant at the end of the study period ( $P=0.045$, Figure 8.7). Neither PDO 1 nor placebo did affect stool frequency or consistency throughout the study period (data not shown).

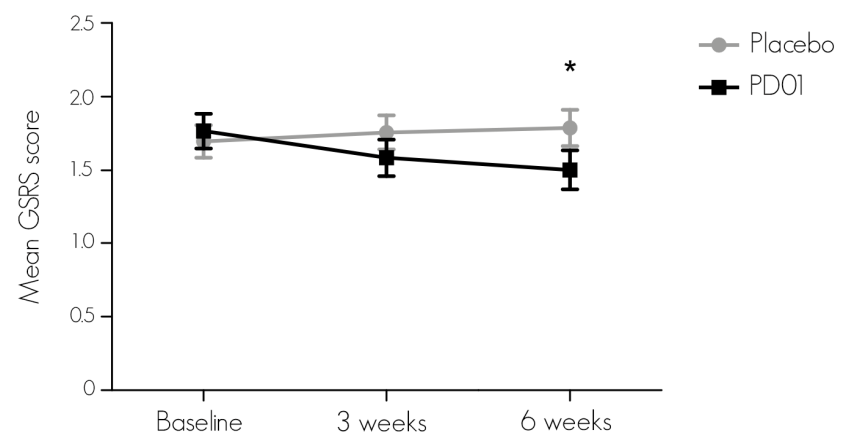

Figure 8.7 Change in GSRS score for indigestion syndrome from baseline to end of the study period. Values are means with their standard errors represented by vertical bars. ${ }^{*} P \leq 0.050$. 


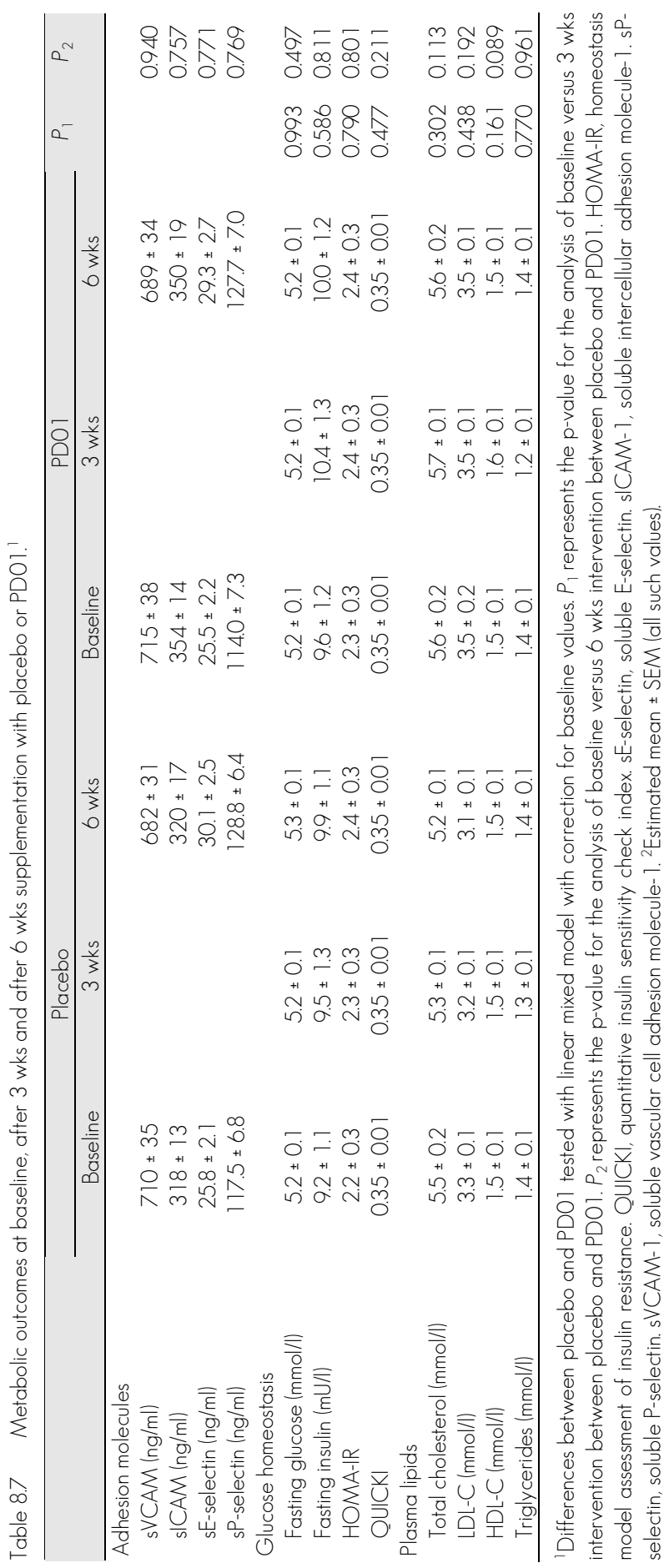




\section{Discussion}

This is the first human study evaluating the intestinal fate of a carotenoid-producing bacillus strain as well as its potential Gl and systemic effects. We have shown that PDO1 survived transit through the $\mathrm{G}$ tract and was able to germinate into vegetative cells under intestinal conditions and release carotenoids in situ. The latter were shown to have excellent bioavailability, with levels in blood at the end of the study in similar ranges as plant carotenoids. Repeated intake of PDO1 was well tolerated and was even associated with a significant improvement of indigestion symptoms. In this first explorative study with obese, yet healthy volunteers, no significant effects on gut microbiota and barrier function, nor systemic effects on antioxidant activity, oxidative stress, adhesion molecules, glucose and insulin homeostasis, blood lipids and BP could be observed.

PDO1 survived passage through the human GI tract and daily PDO1 supplementation over a period of 6 wks led to a significant accumulation of PDO1 carotenoids in plasma. PDO 1 carotenoids were detected in all subjects supplemented with PDO1, demonstrating absorption and transport to the systemic circulation. Plasma concentrations of PDO1 carotenoids were lower in comparison with those of some of the dietary carotenoids, but it should be taken into account that intake of these dietary carotenoids is of a much higher magnitude. On the other hand, intestinal absorption of bacterial carotenoids appears to be more efficient than that of dietary carotenoids. More specifically, the average lutein intake is estimated at 1-3 mg/day, ${ }^{32}$ with plasma lutein concentrations of $0.12-0.13 \mu \mathrm{M}$. The calculated intake of PDO 1 carotenoids was only $0.014 \mathrm{mg} /$ day with plasma concentrations of 0.04-0.08 $\mu \mathrm{M}$. Despite the almost 100-fold lower intake of PD01 carotenoids, plasma levels were therefore only 2-fold lower as compared to lutein. No changes in plasma concentrations of dietary carotenoids were observed, indicating that these novel PDO1 carotenoids do not compete with other carotenoids for absorption and transport.

In vitro, PDO 1 was shown to modulate the microbial intestinal environment by increasing the presence of two bacterial groups, namely the firmicutes and Clostridium coccoides/Eubacterium rectale groups. In this human intervention study we did not observe a significant effect of PDO1, compared to placebo, on gut microbiota composition. No significant changes in the relative abundance of the dominant phyla or on the microbial composition at genus level were observed between groups. However, when analyzing the microbial composition over time separately in the PDO1 group, significant increases in the fecal presence of several bacteria belonging to the lachnospiraceae and ruminococcaceae (part of the firmicutes) were observed, while in the placebo group no alterations in these species were detected. The lachnospiraceae and ruminococcaceae groups include many butyrate-producers, and an increase might be beneficial as butyrate plays a key role in maintaining gut homeostasis and epithelial integrity. ${ }^{33}$ 
In vitro, PDO I also altered SCFA profiles by inducing a shift from acetate to butyrate and by increasing lactate production. Here, no significant alterations were observed after PDO 1 vs. placebo supplementation. In both treatment groups, baseline stool pH was within normal ranges and did not significantly change after treatment. Additionally, SCFA production, monitored as a marker of saccharolytic fermentation, was stable throughout the study period and between groups. We also determined $\beta$-glucuronidase activity in the fecal samples, but found no between-group differences after 6 wks supplementation. High microbial $\beta$-glucuronidase activity has been associated with development of colon cancer as it may transform pro-carcinogenic substances carcinogenic. ${ }^{34}$ On the other hand, high microbial $\beta$-glucuronidase activity is considered beneficial as it is associated with an increase in butyrate-producing microorganisms from the firmicutes phylum, particularly within clostridial clusters XIVa and IV.34

With respect to PDO 1-induced effects on the gut metabolome: PCA score plots revealed that interindividual variation caused by genomic variation and exposome influence le.g. lifestyle factors ${ }^{35}$ ) were more dominant than potential bacillus-induced metabolomic effects, as analyzed by hierarchical clustering analysis. The best option to visualize the bacillusinduced changes would be to correct for the confounding factors lgenetics, dietary factors, etc.) and to create a plot per individual. One could question the coverage of the detected metabolome but this proved more than adequate, as over $30 \%$ of the detected metabolites proved significantly altered when implementing the metabolomics fingerprinting approach on digestive fluid samples derived from in vitro gut simulations (unpublished data).

The above-discussed results of PDO1 supplementation suggest that the current human study lacked sufficient resolution to confirm previously obtained in vitro and in vivo data. On the other hand, the obtained data should be interpreted as positive because a metabolic effect has been established (carotenoid production) without significantly disturbing microbiota composition or intestinal homeostasis of gut metabolomics. Indeed, the low incidence of Gl complaints underpins the excellent tolerability of PDO 1 in our healthy obese individuals.

Furthermore, in addition to being well tolerated, the daily intake of PDO1 for 6 wks even resulted in a significant decrease in $G$ symptoms of indigestion, such as borborygmi, abdominal distension, eructation and increased flatulation. This is in line with literature reports on (potential) pre-, pro- or sybiotics in healthy individuals or individuals with irritable bowel syndrome (IBS). ${ }^{36-38}$ A possible explanation for a decrease in such symptoms is a $\mathrm{Gl}$ decrease in bacterial groups with gas-producing abilities. ${ }^{39,40}$

Probiotics are suggested to improve Gl permeability by stimulation of the intestinal mucosal defence. $^{41}$ The only available human intervention trial that evaluated the effect of a probiotic on $\mathrm{Gl}$ permeability in similar subjects did not provide evidence for an improvement. ${ }^{42}$ Interestingly, our findings suggest that $\mathrm{Gl}$ effects of PDOl extend to improvement of $\mathrm{Gl}$ permeability. While between group effects in $\mathrm{Gl}$ permeability did not 
reach significance, an indication towards improvement in gastroduodenal and colonic gut permeability was noted, as assessed with a validated multi-sugar test.

As PDO1 produces carotenoids that have high structural similarity to lycopene, our study findings related to CVD risk markers should be compared with studies on lycopene.

Human intervention studies have shown that daily consumption of lycopene (20-30 mg/day; a dose 1000-fold higher than PDO1 in this study) exerts beneficial effects on biomarkers of oxidative stress in healthy volunteers. ${ }^{43}$ In our study, the bacterial carotenoids did not induce significant changes in TAC, urinary $F_{2}$-isoprostanes excretion or MDA. Baseline TAC values of our study population were however found to be higher than expected for overweight and obese subjects, ${ }^{44}$ potentially masking beneficial effects of PDO 1.

The presence of PDO1 carotenoids did not influence circulating adhesion molecules concentrations. These proteins, expressed on the vascular endothelium and on circulating leukocytes, mediate inflammatory processes and play an important role in the development and stability of atherosclerotic plaques. ${ }^{45}$ Plasma concentrations reflect the degree of endothelial function. In our study, baseline plasma adhesion molecules values were comparable to those reported for similar study populations using the same analysis. ${ }^{46}$ Human data among the effects of lycopene on plasma adhesion molecules are scarce and contradictory. ${ }^{47,48}$

Obesity may be associated with a wide cluster of metabolic alterations, including glucose homeostasis disorders and dyslipidemia. In animal studies, lycopene proved to decrease serum glucose, reduce total and LDL-cholesterol and to increase HDL-cholesterol. ${ }^{49,50}$ In humans, results regarding the beneficial effects of lycopene on these parameters are not uniform. $^{51,52}$ Supplementing the carotenoid-producing bacillus strain PDO1 to overweight and obese, but otherwise healthy volunteers, did not affect systemic glucose, insulin and lipid levels. In retrospect, since the participants had completely normal values at baseline, no significant improvement through dietary intervention was to be anticipated.

The BP lowering effect of lycopene, and of carotenoids in general, is supported by several, ${ }^{53,54}$ yet not all, ${ }^{55}$ human intervention trials. Carotenoids have been demonstrated to scavenge free radicals and to lower levels of reactive oxidative species, thus lowering oxidative stress. This may prevent oxidation of LDL-cholesterol, which in turn can trigger a cascade of events associated with atherogenesis. ${ }^{56}$ Furthermore, oxidized LDL particles impair normal endothelial function by inhibiting nitric oxide release, an important vasodilator, and thus affect BP. ${ }^{57}$ In our intervention study, no effects of PDO 1 treatment on systolic and diastolic BP were observed compared to placebo.

Some potential shortcomings of our study design should be mentioned. Firstly, with most baseline parameters within normal ranges, the induction of systemic beneficial changes in generally healthy subjects through dietary supplementation is challenging and when present often only marginal. Another possible limitation is the lack of a well-controlled diet. We instructed participants to maintain their habitual dietary intake throughout the study period, 
as we aimed to assess the effects of PDOI as supplement to their habitual diet, which is in line with future applications. Based on the unchanged plasma concentrations of lutein, lycopene, and $\beta$-carotene it can be assumed that the habitual diet was indeed maintained. We cannot exclude that the presence of dietary carotenoids may have masked the additive effect of the bacterial carotenoids.

In conclusion, this study provides the first evidence in humans that PDOI survives transit through the $\mathrm{Gl}$ tract and is able to germinate and release bacterial carotenoids, which are absorbed and detected in human blood plasma. Furthermore, even though systemic effects of the carotenoids could not be confirmed in this explorative study, repeated intake improved local Gl parameters. These promising results warrant further research in specific target populations to investigate the specific bioactivities of PDO 1 in the intestine and on systemic parameters. 


\section{References}

1. Serra-Majem L., Roman B, Estruch R. Scientific evidence of interventions using the Mediterranean diet: a systematic review. Nutr Rev. 2006;64(2 Pt 2):S27-47.

2. Krinsky $\mathrm{NI}$, Johnson EJ. Carotenoid actions and their relation to health and disease. Mol Aspects Med. 2005;26(6):459-516.

3. Hozawa A, Jacobs DR Jr, Steffes MW, et al. Relationships of circulating carotenoid concentrations with several markers of inflammation, oxidative stress, and endothelial dysfunction: the Coronary Artery Risk Development in Young Adults (CARDIA)/Young Adult Longitudinal Trends in Antioxidants (YALTA) study. Clin Chem. 2007;53(3):447-55

4. Rao AV, Rao LG. Carotenoids and human health. Pharmacol Res. 2007;55(3):207-16.

5. Krinsky NI. The antioxidant and biological properties of the carotenoids. Ann N Y Acad Sci. 1998;854: 443-7.

6. Rice-Evans CA, Sampson J, Bramley PM, Holloway DE. Why do we expect carotenoids to be antioxidants in vivo? Free Radic Res. 1997;26(4):38 1-98.

7. Khaneja R, Perez-Fons L, Fakhry S, et al., Carotenoids found in Bacillus. J Appl Microbiol, 2010. 108(6): p. 1889-902.

8. Duc le H(1), Fraser PD, Tam NK, Cutting SM. Carotenoids present in halotolerant Bacillus spore formers. Fems Microbiology Letters. 2006;255(2):215-224.

9. Hong HA, Huang JM, Khaneja R, et al. The safety of Bacillus subtilis and Bacillus indicus as food probiotics. J Appl Microbiol. 2008; 105(2):510-20.

10. Ley RE. Obesity and the human microbiome. Curr Opin Gastroenterol. 2010;26(1):5- 11.

11. Turner JR. Intestinal mucosal barrier function in health and disease. Nat Rev Immunol. 2009;9(1 1): 799-809.

12. Cani PD, Amar J, Iglesias MA, et al. Metabolic endotoxemia initiates obesity and insulin resistance. Diabetes. 2007;56(7): 176 1-72.

13. de La Serre CB, Ellis CL, Lee J, et al. Propensity to high-fat diet-induced obesity in rats is associated with changes in the gut microbiota and gut inflammation. Am J Physiol Gastrointest Liver Physiol. 2010;299(2):G440-8.

14. Campia U, Tesauro M, Cardillo C. Human obesity and endothelium-dependent responsiveness. Br J Pharmacol. 2012; 165(3):561-73.

15. Kang YS. Obesity associated hypertension: new insights into mechanism. Electrolyte Blood Press. 2013; $11(2): 46-52$.

16. Kahn SE, Hull RL, Utzschneider KM. Mechanisms linking obesity to insulin resistance and type 2 diabetes. Nature. 2006;444(7121):840-6.

17. Franssen R, Monajemi H, Stroes ES, Kastelein JJ. Obesity and dyslipidemia. Med Clin North Am. 2011 ; 95(5):893-902.

18. Gleize B, Steib M, André M, Reboul E. Simple and fast HPLC method for simultaneous determination of retinol, tocopherols, coenzyme $\mathrm{Q}(10)$ and carotenoids in complex samples. Food Chem. 2012;134(4): 2560-4.

19. Hevia A, Bernardo D, Montalvillo E, et al. Human colon-derived soluble factors modulate gut microbiota composition. Front Oncol. 2015;5:86.

20. Milani C, Hevia A, Foroni E, et al. Assessing the fecal microbiota: an optimized ion torrent 165 rRNA genebased analysis protocol. PLoS One. 2013;8(7):e68739.

21. Caporaso JG, Kuczynski J, Stombaugh J, et al. OlIME allows analysis of high-throughput community sequencing data. Nature Methods. 2010;7(5):335-6.

22. Edgar, R.C. Search and clustering orders of magnitude faster than BLAST. Bioinformatics. 2010; 26(19):2460-1. 
23. Quast C, Pruesse E, Yilmaz P, et al. The SILVA ribosomal RNA gene database project: improved data processing and web-based tools. Nucleic Acids Res. 2013;41(Database issue):D590-6.

24. Lozupone C, Knight R. Unifrac: a new phylogenetic method for comparing microbial communities. Applied and Environmental Microbiology. 2005;71(12):8228-8235.

25. de Wiele TV, Boon N, Possemiers S, et al. Prebiotic effects of chicory inulin in the simulator of the human intestinal microbial ecosystem. FEMS Microbiol Ecol. 2004;51 11):143-53.

26. Vanden Bussche J, Marzorati M, Laukens D, Vanhaecke L. Validated High Resolution Mass SpectrometryBased Approach for Metabolomic Fingerprinting of the Human Gut Phenotype. Anal Chem. 2015;87(21): 10927-34

27. Mujagic Z, Ludidi S, Keszthelyi D, Hesselink MA, et al. Small intestinal permeability is increased in diarrhoea predominant IBS, while alterations in gastroduodenal permeability in all IBS subtypes are largely attributable to confounders. Aliment Pharmacol Ther. 2014;40(3):288-97.

28. van Wijck K, van Eijk HM, Buurman WA, et al. Novel analytical approach to a multi-sugar whole gut permeability assay. J Chromatogr B Analyt Technol Biomed Life Sci. 201 1;879(26):2794-801.

29. Svedlund J, Siodin I, Dotevall G. GSRS--a clinical rating scale for gastrointestinal symptoms in patients with irritable bowel syndrome and peptic ulcer disease. Dig Dis Sci. 1988;33(2):129-34.

30. Riegler G, Esposito I. Bristol scale stool form. A still valid help in medical practice and clinical research. Tech Coloproctol. 2001;5(3): 163-4.

31. Visioli F, Riso P, Grande S, et al. Protective activity of tomato products on in vivo markers of lipid oxidation. Eur J Nutr. 2003;42(4):201-6.

32. Abdel-Aal el-SM, Akhtar H, Zaheer K, Ali R. Dietary sources of lutein and zeaxanthin carotenoids and their role in eye health. Nutrients. 2013;5(4): $1169-85$

33. Hamer HM, Jonkers D, Venema K, et al. Review article: the role of butyrate on colonic function. Aliment Pharmacol Ther, 2008;27(2): 104-19.

34. Dabek M, McCrae SI, Stevens VJ, et al. Distribution of beta-glucosidase and beta-glucuronidase activity and of beta-glucuronidase gene gus in human colonic bacteria. FEMS Microbiol Ecol. 2008;66(3):487-95.

35. Wild CP, Scalbert A, Herceg Z. Measuring the exposome: a powerful basis for evaluating environmental exposures and cancer risk. Environ Mol Mutagen. 2013;54(7):480-99,

36. Kalman DS, Schwartz HI, Alvarez P, et al. A prospective, randomized, double-blind, placebo-controlled parallel-group dual site trial to evaluate the effects of a Bacillus coagulans-based product on functional intestinal gas symptoms. BMC Gastroenterol. 2009:9:85.

37. Cappello C, Tremolaterra F, Pascariello A, et al. A randomised clinical trial (RCT) of a symbiotic mixture in patients with irritable bowel syndrome (IBS): effects on symptoms, colonic transit and quality of life. Int J Colorectal Dis. 2013;28(3): 349-58.

38. Silk DB, Davis A, Vulevic J, et al. Clinical trial: the effects of a trans-galactooligosaccharide prebiotic on faecal microbiota and symptoms in irritable bowel syndrome. Aliment Pharmacol Ther. 2009;29(5):508- 18.

39. Johansson ML, Molin G, Jeppsson B, et al. Administration of different Lactobacillus strains in fermented oatmeal soup: in vivo colonization of human intestinal mucosa and effect on the indigenous flora. Appl Environ Microbiol. 1993;59(1): 15-20

40. Quigley EM. From comic relief to real understanding; how intestinal gas causes symptoms. Gut. 2003; 52(12): 1659-61.

41. Gionchetti P, Rizzello F, Campieri M. Probiotics in gastroenterology. Curr Opin Gastroenterol. 2002; 18(2):235-9.

42. Leber B, Tripolt NJ, Blattl D, et al. The influence of probiotic supplementation on gut permeability in patients with metabolic syndrome: an open label, randomized pilot study. Eur J Clin Nutr. 2012;66(10): 1110 -5.

43. Devarai S, Mathur S, Basu A, et al. A dose-response study on the effects of purified lycopene supplementation on biomarkers of oxidative stress. J Am Coll Nutr. 2008;27(2):267-73.

44. Chrysohoou C, Panagiotakos DB, Pitsavos C, et al. The implication of obesity on total antioxidant capacity in apparently healthy men and women: the ATTICA study. Nutr Metab Cardiovasc Dis. 2007; 17(8):590-7. 
45. Palmefors $H$, DuttaRoy S, Rundqvist B, Böriesson $M$. The effect of physical activity or exercise on key biomarkers in atherosclerosis--a systematic review. Atherosclerosis. 2014;235(1): 150-61.

46. Basu A, Betts NM, Nguyen A, et al. Freeze-dried strawberries lower serum cholesterol and lipid peroxidation in adults with abdominal adiposity and elevated serum lipids. J Nutr. 2014; 144(6):830-7.

47. Blum A, Monir M, Khazim K, et al. Tomato-rich (Mediterranean) diet does not modify inflammatory markers. Clin Invest Med. 2007;30(2):E70-4.

48. García-Alonso FJ, Jorge-Vidal V, Ros G, Periago MJ. Effect of consumption of tomato juice enriched with n-3 polyunsaturated fatty acids on the lipid profile, antioxidant biomarker status, and cardiovascular disease risk in healthy women. Eur J Nutr. 2012;51(4):415-24.

49. Ali MM, Agha FG. Amelioration of streptozotocin-induced diabetes mellitus, oxidative stress and dyslipidemia in rats by tomato extract lycopene. Scand J Clin Lab Invest. 2009;69(3):371-9.

50. Lorenz M, Fechner M, Kalkowski J, et al. Effects of lycopene on the initial state of atherosclerosis in New Zealand White (NZW) rabbits. PLoS One. 2012;7(1):e30808.

51. Thies $F$, et al. Effect of a tomato-rich diet on markers of cardiovascular disease risk in moderately overweight, disease-free, middle-aged adults: a randomized controlled trial. Am J Clin Nutr. 2012; 95(5): 1 13-22.

52. Collins JK, Arimandi BH, Claypool PL, et al. Lycopene from two food sources does not affect antioxidant or cholesterol status of middle-aged adults. Nutr J. 2004;3:15.

53. Kim JY, Paik JK, Kim OY, et al. Effects of lycopene supplementation on oxidative stress and markers of endothelial function in healthy men. Atherosclerosis. 201 1;215(1): 189-95.

54. Engelhard YN, Gazer B, Paran E. Natural antioxidants from tomato extract reduce blood pressure in patients with grade- 1 hypertension: a double-blind, placebo-controlled pilot study. Am Heart J. 2006; 151(1): 100.

55. Ried K, Frank OR, Stocks NP. Dark chocolate or tomato extract for prehypertension: a randomised controlled trial. BMC Complement Altern Med. 2009:9:22.

56. Basu A, Imrhan V. Tomatoes versus lycopene in oxidative stress and carcinogenesis: conclusions from clinical trials. Eur J Clin Nutr. 2007;61(3):295-303.

57. Willcox JK, Catignani GL, Lazarus S. Tomatoes and cardiovascular health. Crit Rev Food Sci Nutr. 2003; 43(1): 1- 18 


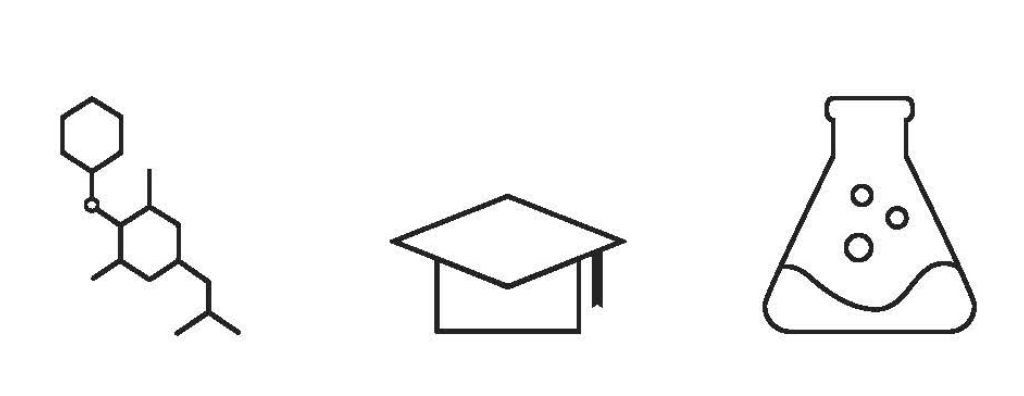




\section{chapter 9}

\section{General discussion}




\section{Background}

The relationship between food and health is complex, being multifactorial and multidimensional. Food contributes to human health and enhances the health potential, but can also be a key actor negatively influencing health and initiating or contributing to illness. The primary role of food is to provide sufficient nutrients to meet the nutritional requirements of an individual. Furthermore, food supplies the body with materials that can be used for the construction and repair of body tissues. This allows an individual to grow and thrive. Recently, there has been a growing interest in the health-enhancing role of specific foods in addition to their nutritional value. Efforts to identify functional and bioactive components from many natural sources have been intensified and have eventually led to a new group of products, the so-called functional foods. A food can be classified as "functional" when it contains a component (whether a nutrient or not) that affects one or more functions in the body in a targeted way so that positive effects on health' are to be expected, or when the component has a physiological or psychological effect beyond the traditional nutritional effect. ${ }^{2}$ Functional foods may originate from plant and animal sources, from microorganisms or marine organisms.

In many low- and middle income countries access to food, availability and quality of food/nutrition are major concerns. To date, worldwide, approximately 800 million individuals are plagued by undernourishment and food insecurity. ${ }^{3}$ These individuals, often children, consume insufficient protein and energy, and the adverse health effects of this are often compounded by deficiencies of vitamins and minerals. In these countries, easy-to-remedy nutritional deficiencies prevent 1 of 38 newborns from reaching an age of 5 years. ${ }^{4}$ In contrast, the prevalence of overweight and obesity, linked to excess food intake and overnutrition, has more than doubled since 1980. In 2014, worldwide, more than 1.9 billion adults aged 18 years and older were overweight, and of these, over 600 million were obese. $^{5}$ Overweight and obesity are associated with alterations in gut microbiota composition and activity, , impaired gut barrier function, $^{8-10}$ gastrointestinal and systemic low-grade inflammation, ${ }^{11-14}$ impaired vascular functions, 15,16 and glucose and lipid homeostasis disorders. ${ }^{17,18}$ These gastrointestinal and systemic metabolic abnormalities are major risk factors for the development of diabetes mellitus type 2 (DM2) and cardiovascular diseases (CVD). ${ }^{19-21}$ Worldwide, overweight and obesity now cause more deaths than underweight. Consequently, obesity and related illnesses have an enormous health and economic impact on society. 22 


\section{Nutritional interventions in healthy, overweight and obese individuals}

The aim of this thesis was to investigate the effects of several nutritional interventions on gastrointestinal and metabolic health in healthy, overweight and obese individuals. This thesis presents the results of six randomized-controlled trials. The nutritional interventions we studied included a prolyl endoprotease, a putative prebiotic supplement, a putative probiotic species, and two different phytonutrients.

\section{Gastrointestinal gluten degradation by a prolyl endoprotease}

Ingestion of gluten - a storage protein present in wheat, barley and rye - can induce a wide range of gluten related disorders with different pathogenic pathways, globally affecting a large proportion of the population. ${ }^{23,24}$ In subjects suffering from celiac disease and wheat allergy, the presence of gluten peptides in the small intestine triggers an abnormal immune response resulting in intestinal inflammation and ultimately villous atrophy and crypt hyperplasia. ${ }^{25}$ The mechanisms by which gluten induce symptoms in subjects with non-celiac gluten sensitivity remain largely unknown, but involvement of the innate immune system has been well established. ${ }^{26,27}$ For all conditions, strict adherence to a gluten-free diet is the only effective treatment. However, the high cost, poor availability and poor palatability of gluten-free products makes it difficult to strictly adhere to such diet on the longer term. ${ }^{28}$ In addition, gluten-free food products available on the market often contain trace amounts of gluten. ${ }^{29}$ When maintained appropriately, a gluten-free diet is not nutritionally optimal; it may predispose to an undesirable high intake of fat and carbohydrates and lead to poor vitamin status and detrimental weight gain or weight loss. ${ }^{30}$ Due to the consequences of gluten-free dieting, an unmet need for alternative therapies exists. Several options have been investigated. One approach is to use compounds that do not have the immunogenic proteins found in wheat derived gluten. Several wheat substitutes are available, including sorghum, almonds, rice, corn and legumes. ${ }^{25,31}$ Another option is selecting and breeding wheat products that only contain low amounts of T-cell stimulatory sequences, thereby reducing the immunotoxic effects. ${ }^{32}$ Furthermore, developing genetically modified gluten with reduced immunogenicity might be a potential future option for celiac disease patients. By blocking glutamine residues, the affinity of gluten peptides to bind to DO2 and DQQ molecules is reduced, resulting in a reduced T-cell-mediated inflammatory response. ${ }^{33}$ Wheat fermentation with sourdough lactobacilli and fungal proteases has also been shown to reduce wheat toxicity. ${ }^{34-36}$ However, as gluten protein is important in baking due to its strengthening and binding properties, it will be difficult to alter or select a variety of wheat completely devoid of gluten without compromising its mechanical properties. Binding glutens to polymers in the gastrointestinal tract may also prevent degradation, absorption and 
triggering of an immunologic response. ${ }^{37}$ Another interesting alternative treatment is oral enzyme supplementation to degrade gluten peptides. Food-derived gluten contains considerable amounts of proline and glutamine amino acids, which are highly resistant to enzymatic proteolysis within the human digestive tract. ${ }^{38}$ Prolyl endoproteases are enzymes that are able to target and destroy proline and glutamine residues into small nontoxic gluten peptides before they enter the intestinal epithelium. ${ }^{39}$ In Chapter 2 we studied the in vivo gluten-degrading potential of Aspergillus Niger-derived prolyl endoprotease (AN-PEP). ANPEP showed to efficiently degrade gluten from a meal in the stomach of healthy volunteers, within the time period a meal normally resides in the stomach. These in vivo results confirmed previously obtained in vitro observations. ${ }^{40,41}$ In addition, we hypothesized that increasing the caloric density of a meal enhances gluten degradation by delaying gastric emptying rate, thereby prolonging exposure time of gluten proteins to AN-PEP, endogenous proteases and gastric acid. Indeed, increasing meal caloric density delayed gastric emptying and prolonged the gastric residence time of AN-PEP, but it did not affect its efficiency in degrading gluten. To standardize each meal intake we intragastrically administered the test meal via a triple lumen nasogastroduodenal tube, this obviously does not represent a fully physiological meal setting. Thus, data on the gluten-degrading potential of AN-PEP in an actual meal setting in gluten-intolerant subjects are needed. Also microbial prolyl endopeptidases from other species, as well as germinating cereal enzymes, have shown to possess the capacity to hydrolyze gluten peptides in vitro ${ }^{42-44}$ and in vivo. ${ }^{45-47} \mathrm{~A}$ combination of both, known as ALVOO3, already proved that it is able to attenuate gluteninduced mucosal injury in celiac disease patients. ${ }^{46}$

In summary, enzyme supplementation therapy targeted at degradation of immunogenic gluten peptides appears to be a promising approach. Development of such a supplement as alternative for a gluten-free diet is still in its early phase of validations and subsequent implementation. It is anticipated that enzymes first will be marketed as a supplement 1) when ingested in combination with a gluten-free diet, 2) when ingested with a normal gluten containing meal, for social reasons or 3) in conditions where persons report gluten hypersensitivity in absence of celiac disease.

\section{Food-drug interaction}

To exert a systemic effect, a compound has to be absorbed from the gastrointestinal tract. The onset of a systemic effect heavily depends on the rate of absorption, which is in part determined by the gastric emptying rate. In Chapter 3 we critically evaluated a wellestablished method of measuring gastric emptying, namely by measuring plasma concentrations of paracetamol. It is assumed that paracetamol is absorbed almost immediately after reaching the small intestine and that the transit time of paracetamol through the stomach is identical to that of the water phase of a meal. Thus, gastric emptying 
rate might be derived from the time course of plasma paracetamol appearance. ${ }^{48,49}$ However, in Chapter 3, we demonstrated that paracetamol when added to a meal and mixed, was divided in two fractions. One fraction was dissolved in the liquid content of the meal which quickly left the stomach and was absorbed in the duodenum, reflected by an early, high plasma peak. A second paracetamol fraction was encapsulated by the meal, leaving the stomach more slowly and was even not taken up instantaneously after reaching the intestine. Both fractions did not accurately reflect gastric emptying rate. Additionally, this showed us that the rate-limiting factor for absorption was actually the bioaccessibility, i.e. release of paracetamol from the food matrix. The food matrix and structure can have both positive and negative effects on bioaccessibility. ${ }^{50}$ Thus, it is important to realize that fooddrug interactions are not limited to the effect of food on gastric emptying alone.

\section{The human gut microbiome}

The human gut microbiome harbors several trillion microbes residing in the gut. ${ }^{51}$ It serves a wide array of functions, including regulating normal functioning of the gastrointestinal tract, harvesting energy from indigestible parts of the diet, maintaining intestinal epithelial integrity, metabolizing indigestible polysaccharides, absorbing short-chain fatty acids (SCFA) produced by bacterial fermentation and regulating intestinal transit. $10,52-55$ The colonization of the human gut by micro-organisms starts at gestation, during birth and immediately afterwards. Bacteria from the mother and surrounding environment colonize the infants gut. It is affected by several factors such as genetic background, gestational age, mode of delivery, type of feeding, exposure to antimicrobials and, very important, the diet throughout life. ${ }^{56}$ Long-term perturbations to the intestinal ecosystem can induce changes in the microbiota, contributing to the development and progression of a number of gastrointestinal disorders ${ }^{57}$ such as irritable bowel syndrome, celiac disease, inflammatory bowel disease and colorectal cancer, as well as systemic diseases such as allergic diseases, ${ }^{58}$ non-alcoholic steatohepatitis, ${ }^{59}$ obesity ${ }^{54,60,61}$ and diabetes. ${ }^{62}$ Thus, targeting the microbiome seems to offer a promising approach to potentially ameliorate above mentioned gastrointestinal and metabolic diseases. A number of dietary strategies are available for modulating the composition and function of the intestinal microbiota; pre- and probiotics are among the most well established.

\section{Gut microbiota, gut barrier and immune modulation by prebiotics}

Prebiotics are non-digestible compounds that selectively stimulate growth and/or activity of one or a limited number of microbial species in the gut with potential health benefits to the host. $^{63}$ Many animal studies showed positive results of prebiotics in the prevention or treatment of gastrointestinal and chronic metabolic diseases, but human data remain 
ambiguous. ${ }^{64}$ SCFA are produced by bacterial fermentation of non-digestible compounds. These metabolites display several important functions; they maintain energy homeostasis, are able to affect cell proliferation and differentiation, have anti-inflammatory and immunomodulating effects, serve as an energy source for colonocytes and are essential in establishing and maintaining the gut barrier by regulating expression of tight junction proteins. $^{65-67}$ Furthermore, high amounts of intestinal SCFA lower intestinal pH, affect microbiota composition by inhibiting the growth of pathogenic bacteria, and creating a more favourable environment for the growth of beneficial bacteria. ${ }^{68,69}$ Most studied prebiotics are fructooligosaccharides, galactooligosaccharides, inulin and resistant starch. These prebiotics are predominantly fermented in the more proximal colon and are limited in reaching the distal colon. ${ }^{70,71}$ However, several chronic colonic diseases originate from the distal colon; consequently, there is a great interest in finding prebiotics that are able to reach, are fermented, and act in the more distal regions of the colon. Arabinoxylans (AX) form an interesting novel class of such type prebiotics, as they rely on a whole spectrum of microbial enzymes for their degradation, resulting in a more distal fermentation and action. $^{72-74}$ In Chapter 4 we have described the results of a human study supplementing an AX extract to overweight and obese individuals for several weeks. Previous in vitro and animal studies already had shown that this specific extract was able to modulate gut microbiota composition and microbial activity and, as a consequence, gut barrier function. $70,75-77$ In our in vivo study the overall microbiota composition of participants did not significantly change following AX treatment, but increases in fecal SCFA concentrations and a decrease in fecal $\mathrm{pH}$ were observed.

Modulation of gut microbiota by prebiotics has been associated with improvements in gut barrier function. The exact underlying mechanisms are poorly understood. An increased villus height and crypt depth, a thickened mucosal layer, increased transcription and consequently increased synthesis of tight junction proteins, and a change in the distribution and localization of tight junction proteins may contribute. ${ }^{9,78-81}$ The integrity of the gastrointestinal epithelium depends on formation of adhesive contacts between neighbouring epithelial cells. This is mediated by the apical junctional complex, which is composed of the tight junction and subjacent adherence junction. These multi-protein complexes are considered critical regulators of the gastrointestinal barrier function. Adherence junctions are required for assembly of the tight junction, which seals the paracellular space. Tight junction proteins consist of transmembrane proteins, peripheral membrane (scaffolding) proteins and regulatory molecules. The two major tight junction transmembrane proteins are the claudins and occludins. Peripheral membrane proteins, such as members of the zonula occludens family, function as scaffolding proteins between transmembrane and cytoplasmic proteins. In addition, it has been proposed that these proteins link adherence junctions to tight junctions through protein linkages. ${ }^{82,83}$ In Chapter 4, AX showed to affect gut barrier function; as upregulation in the transcription of the tight 
junction proteins claudin-3, claudin-4 and occludin in sigmoid biopsies was observed. The changes in tight junction gene expression were not accompanied with changes in mucosal tight junction protein expression or in gastrointestinal permeability as assessed with a functional test.

Another important function of the colonic microbiota is its role in the development and maturation of the immune system. The gut contains an important component of the body's immune system, called gut-associated lymphoid tissue. The immune system must establish an appropriate balance between tolerance to the gut commensal microbiota and vigilance to guard against infectious agents and opportunistic pathogens. The cross-talk between the intestinal microbiota and host involves both the innate and adaptive immune system. Imbalances in gut microbiota and consequently in immune homeostasis may lead to a number of pathologies, such as obesity, DM2, inflammatory bowel disease and colorectal cancer. ${ }^{84}$ Previous studies indicate that prebiotics can exert direct immunomodulatory effects $^{85,86}$ and indirect immunomodulatory effects through changing the gut microbiome. ${ }^{87}$ Microbiota-dependent changes affecting the immune system include stimulation of the growth and metabolism of protective commensal intestinal bacteria. An increase in the presence of beneficial bacteria, such as bifidobacteria and lactobacilli, will provide antimicrobial effects by direct competition with pathogenic bacteria for available binding sites on the intestinal epithelium and for nutrients. ${ }^{88,89}$ Furthermore, some bacterial species are able to produce antibacterial substances that can inhibit the growth and survival of pathogens. $^{90}$ In addition, SCFA might have direct immunomodulatory properties. ${ }^{91-93}$ To assess the effects of $A X$ on immune response, we applied an ex vivo model using PHAstimulated whole blood to evaluate the capacity to produce cytokines (Chapter 4). With AX treatment we observed a significantly decreased production of the pro-inflammatory cytokine TNFa, suggesting a capacity to reduce the intensity of an acute pro-inflammatory reaction. As SCFA are able to suppress the production of such cytokines, the decrease in TNFa production might be linked to the observed increase in fecal SCFA.

Altogether, the data presented in Chapter 4 suggest that $A X$ modulate gut microbial activity and thereby may positively affect gut barrier and immune function.

\section{Gut microbiota and gut barrier modulation by probiotics}

Probiotics are live microorganisms that, when administered in adequate amounts, confer a health benefit on the host. ${ }^{94}$ Most commercial probiotic products contain lactic acid bacteria, such as lactobacilli, streptococci and bifidobacteria. ${ }^{95}$ For a probiotic it is important that it survives the acidic intestinal environment, not being degraded before reaching the site where it is presumed to be active. As with prebiotics, despite convincing and reproducible results from animal studies showing beneficial effects of different probiotic strains on gastrointestinal and metabolic health, the data in humans remain equivocal. ${ }^{64,96} \mathrm{In}$ 
Chapter 8 we studied the effects of a Bacillus indicus strain on the gut microbiota composition and activity in overweight and obese individuals. Bacillus species are grampositive bacteria able to form endospores, i.e. uniquely robust entities that are able to survive extremes of temperature, irradiation and long-term storage. ${ }^{97}$ While some sporeforming species play a role in food poisoning, several species of bacillus are consumed as food ingredients and regarded safe for human consumption. ${ }^{98}$ Bacillus spores are able to pass the gastric barrier without loss of function, are stable to many food processing steps and can be stored at room temperature, making them attractive as a probiotic. Previous (unpublished) in vitro research showed that this carotenoid-producing Bacillus indicus strain was able to survive and germinate in the gastrointestinal tract and to modulate the intestinal environment by increasing the presence of two bacterial groups, namely the firmicutes and Clostridium coccoides/Eubacterium rectale group. Furthermore, it was shown to alter SCFA profiles by inducing a shift from acetate to butyrate, and it increased the lactate production. This shift may provide health benefits for the host. Indeed, in Chapter 8 we demonstrated that in vivo the Bacillus indicus strain was able to survive passage throughout the digestive tract as viable PDOI cells and spores were found in the feces of all individuals supplemented with the strain. However, no significant treatment effects regarding intestinal microbiota composition and microbial activity were observed. We also did not observe modulation of gut barrier function by the Bacillus indicus treatment. The only significant improvement with this Bacillus indicus strain we observed was a decrease in reported gastrointestinal symptoms such as borborygmi, abdominal distension, eructation and increased flatulation. The occurrence of gastrointestinal symptoms was already low at baseline of the study, but significantly reduced after 6 weeks PDOI supplementation compared to placebo. In IBS patients, it has been shown that a difference in gut microbiota composition and activity causes more intestinal gas production, impairs the transit and tolerance of gas, resulting in gastrointestinal symptoms such as bloating. It was also shown that modulation of the gut microbiome by probiotics caused a relief in these symptoms, suggesting that these products are able to induce a quantitative and qualitative gastrointestinal decrease of bacterial groups with gas-producing abilities, such as clostridium species, in the colonic mucosa. ${ }^{99-101}$ Our findings are in line with previous literature reports on effects of (potential) pre-, pro- or synbiotics on symptoms or gastrointestinal function in healthy individuals or individuals with irritable bowel syndrome. ${ }^{102-104}$

\section{Metabolic consequences of gut microbiota modulation}

Alterations in gut microbiota composition have been shown to underlie or influence the development of metabolic disorders. ${ }^{7}$ 105,106 Both in Chapter 4 and Chapter 8 we explored the effect of putative pre- and probiotic products on metabolic markers. Data with respect to beneficial effects on glucose homeostasis and lipid metabolism in animals are widely 
available, ${ }^{75,107-110}$ while human studies investigating these parameters report conflicting results. ${ }^{11-119}$ Both the $A X$ extract and the Bacillus indicus strain did not affect fasting glucose, insulin and lipid concentrations in our study population. However, baseline values were already within the normal ranges, making it difficult to improve these values. Long-term intervention studies in people with pre-diabetes, metabolic syndrome or hypercholesterolemia are required to determine whether pre- or probiotics exert beneficial effects in high-risk individuals.

\section{In vivo bioavailability of hesperidin}

Large epidemiological studies have shown that the intake of fruits and vegetables exerts positive effects on metabolic and cardiovascular health. ${ }^{120-123}$ These effects are partly due to the large amount of polyphenols that these products contain. Hesperidin is such a type polyphenol. It is abundantly present in the peels of citrus fruits. ${ }^{124-129}$ In order to exert systemic health effects, it is essential that a compound is bioavailable and absorbed from the gastrointestinal tract into the systemic circulation. It has been demonstrated that the bioavailability of hesperidin is low in humans, ${ }^{130,131}$ due to a combination of factors. Hesperidin is poorly water soluble $e^{132}$ and it requires to be fermented by colonic microbiota to release and enable the absorption of its aglycone. ${ }^{131,133,134}$ It has been hypothesized that glycosyl hydrolases from the genus bifidobacterium are involved in the hydrolysis of glycolconjugate forms of phytochemicals. ${ }^{135}$ Commercially available hesperidin supplements often elicit less biological effects than natural hesperidin due to a different enantiomer configuration. As a result, several attempts have been made to improve the bioavailability of hesperidin. ${ }^{136-138}$ One way to improve bioavailability is by micronization of this poorly soluble compound. Micronization decreases the particle size resulting in an improved rate of dissolution and thus absorption from the gastrointestinal tract into the systemic circulation. ${ }^{139}$ In Chapter 5 we demonstrated that the bioavailability of a micronized hesperidin $2 \mathrm{~S}$ extract, which has a specific enantiomer configuration similar to natural hesperidin, was improved over that of a non-micronized hesperidin extract with an enantiomer configuration similar to commercially available hesperidin extracts. Improving the bioavailability of hesperidin is important to fully exploit its beneficial properties concerning vascular and metabolic health, as studied in the paragraphs below. Furthermore, we observed considerable inter-individual differences regarding plasma metabolite concentrations. This finding is supported by other human trials investigating the bioavailability of polyphenols. ${ }^{130,140,141}$ As differences in gut microbiota composition between subjects are common, significant inter-individual differences in the microbial transformation of polyphenols will exist. ${ }^{142-144}$ This may serve as an explanation for interindividual differences with respect to biological activity and systemic health effects of a polyphenolic compound. 


\section{The role of hesperidin in vascular health}

Based on the results of Chapter 5, we have chosen the micronized hesperidin 2S formulation to study hesperidin-induced effects on cardiovascular and metabolic parameters in overweight and obese individuals (Chapter 6 and Chapter 7). Hesperidin is considered a strong antioxidant. ${ }^{145,146}$ It has been demonstrated that it increases the production of nitric oxide (NO) in the vascular endothelium by phosphorylation and activation of endothelial $\mathrm{NO}$ synthase (eNOS). ${ }^{126} \mathrm{NO}$ participates in highly active metabolic and regulatory functions including the regulation of endothelial function and blood pressure, and plays a protective role in the development of atherosclerosis. ${ }^{147-150}$ Both in vitro and animal studies have reported consistent, beneficial effects of hesperidin on endothelial function and blood pressure, 126,151,152 while human data is limited and contradicting. ${ }^{125,126}$ As demonstrated in Chapter 6, six weeks daily hesperidin supplementation significantly reduced the levels of circulating adhesion molecules, reflecting an improvement in endothelial function. ${ }^{153}$ Basal flow-mediated dilation (FMD), the gold standard method for measuring endothelial function, ${ }^{154}$ was not improved by hesperidin. However, after inducing temporary endothelial dysfunction, hesperidin protected from postprandial deterioration in FMD in a subgroup of subjects. We excluded subjects with a very low FMD value as it is unlikely that short-term dietary interventions are able to improve such chronically impaired endothelial function ${ }^{155}$ and additionally, the FMD measurement becomes inaccurate. ${ }^{156,157}$ In addition, hesperidin treatment appeared to reduce both systolic and diastolic blood pressure, but this was only significant in the subgroup. As shown in Chapter 7, we did not find any effect of the same hesperidin product on blood pressure after six or twelve weeks daily intake. These contradictory findings reflect the current situation of discrepant results of hesperidin, but also of other polyphenols, on vascular function in humans. ${ }^{142,158}$

\section{Impact of hesperidin on metabolic parameters}

Hesperidin is able to induce glucose lowering effects through altering the transcription of genes encoding regulatory enzymes of glycolysis, gluconeogenesis and glucose utilization in the liver. In addition, it is able to change the expressions of hepatic glucose transporter 2 and adipocyte glucose transporter 4, both playing a critical role in glucose and insulin homeostasis. Furthermore, hesperidin has blood lipid lowering properties, possibly by affecting enzymes involved in lipogenesis, fatty acid biosynthesis, triglyceride synthesis, cholesterol synthesis and cholesterol absorption, and by facilitating fecal triglyceride and cholesterol excretion. ${ }^{159}$ Both in vitro and animal studies have indeed shown beneficial effects of hesperidin on lipids ${ }^{159-162}$ and glucose metabolism. 159,162-164 Positive human results also exist, but only in (pre)diabetic and hyperlipidemic subjects. ${ }^{126,128,165}$ Both in Chapter 6 
and Chapter 7 no blood glucose- or lipid lowering effects of hesperidin treatment were observed. This can be explained by the fact that the participants had normal values from start of the trial. Long-term intervention studies in subjects with (pre)diabetes, metabolic syndrome or hypercholesterolemia are needed to establish and confirm beneficial hesperidin-induced effects on systemic metabolic health markers.

\section{Gut-microbiota dependent effects of polyphenols}

As noted above, the gut microbiome plays a critical role in transforming dietary polyphenols into absorbable biologically active species. Recent data suggests that different types of polyphenolic compounds, including hesperidin, are also able to modulate the gut microbiota composition and function towards a more "health promoting profile.'.66-171 In Chapter 7 we demonstrated that hesperidin did not affect the quantity of SCFA production, but was able to alter the SCFA profile. Hesperidin induced a significant shift from acetate to the healthpromoting SCFA butyrate, possibly via intestinal stimulation of butyrate-producing bacteria. Butyrate functions as an important energy source for colonocytes, exerts anticarcinogenic, anti-inflammatory and antioxidant effects, reinforces the colonic defence barrier, and is able to modulate gut motility and satiety. ${ }^{172}$ These results strengthen the hypothesis that polyphenols are able to modulate gut microbiota composition and function, in turn promoting gut and host health. However, more human intervention studies with a large number of subjects are needed to establish the potential prebiotic-like effects of hesperidin and other polyphenols.

\section{Antioxidant activity of carotenoids}

The incidence of CVD is notably lower in countries with a 'Mediterranean diet', containing a large amount of fruit, vegetables and olive oil. ${ }^{173,174}$ These foods are rich in carotenoids, and a link between carotenoid intake and protection against oxidative stress, atherosclerotic progression, hypertension, inflammation and endothelial dysfunction has been shown. ${ }^{175-177}$ The protection by carotenoids is mainly through their antioxidant activity. They act as antioxidants via several mechanisms. Firstly, they are able to quench highly reactive oxygen species, that have the capacity to oxidize nucleic acids, unsaturated fatty acids or amino acids. ${ }^{178}$ Furthermore, they are able to inhibit free radical reactions, impeding the process of lipid peroxidation, with repair of vitamin $E$ and $C$ radicals. ${ }^{179,180}$ Besides intervening in radical reactions, carotenoids have also been shown to upregulate the antioxidant response element, resulting in another way of protecting cells against highly reactive oxygen species. ${ }^{181}$ The antioxidant capacity of carotenoids is well demonstrated in vitro, ${ }^{178,182}$ but in humans evidence is still lacking that carotenoids act as antioxidants. ${ }^{183}$ Other mechanisms explaining the beneficial effects of carotenoids include their influence on 
gap-junction communication, gene function regulation, hormone and immune modulation, and modulation of drugs metabolism. ${ }^{184-186}$

\section{In vivo bioavailability of bacterial carotenoids}

Humans lack the capacity to synthesize carotenoids by themselves, and therefore depend on dietary intake and subsequent absorption. The majority of commercially available natural carotenoids are derived from plant-based materials, but also some bacterial species are able to synthesize these compounds. The quality and consistency of plant-based carotenoids are dependent on plant growth conditions, which vary tremendously. ${ }^{187,188}$ Furthermore, these carotenoids are degraded in the acidic environment of the stomach. ${ }^{187,188}$ Bacterial carotenoids, produced by spore-forming bacillus strains, deliver gastric-stable carotenoids which are equally or more bioaccessible and bioavailable than plant-based carotenoids. In Chapter 8 we studied the in vivo bioavailability of bacterial carotenoids in overweight and obese subjects. After supplementing the participants with a carotenoid-producing bacillus strain for six weeks, we were able to confirm that bacterial carotenoids were present in the plasma and levels increased throughout the study period. This indicates that the bacillus strain produces in vivo bacterial carotenoids, which are absorbed and transported to the systemic circulation. Absorption of plant-based carotenoids occurs once carotenoids are released from the food matrix. Dissolution of released carotenoids in bulk lipid droplets is followed by the formation of micelles and uptake of these micelles by duodenal mucosal cells occurs through passive diffusion. The micellular carotenoids are then incorporated into chylomicrons and via the lymphatics released into the circulation. ${ }^{189}$ The mechanism of absorption of these bacterial carotenoids has not been elucidated up to now. Possibly, the mechanism is the same as for lycopene due to the high structural similarity of the bacterial carotenoids with lycopene. ${ }^{190}$ Furthermore, uptake of the bacterial carotenoids did not impede the presence of lutein, $\beta$-carotene and lycopene in plasma. It has been suggested that carotenoids may compete with each other or with other lipid-soluble nutrients for their absorption and metabolism, but there is discrepancy in results from the various studies in humans. $^{191-195}$

\section{Carotenoids in vascular and metabolic health}

Compared to plant-based carotenoids, the bacterial carotenoids were shown to have a ten-fold higher in vitro antioxidant activity. As noted before, the relevance of the antioxidant properties of plant-based carotenoids in vivo remains a matter of debate. The effects of carotenoids on oxidative stress, ${ }^{196-199}$ endothelial function, ${ }^{196,197,200}$ glucose homeostasis, ${ }^{201}$ lipid metabolism ${ }^{197-199,201,202}$ and blood pressure ${ }^{199,201,203}$ in humans also remain contradictory. Most of the beneficial effects were obtained in individuals at risk for 
developing CVD or DM2. In Chapter 8 we also explored the antioxidant effects, and effects on cardiovascular and metabolic parameters of the bacterial carotenoids. No cardiovascular or metabolic effects were observed, probably due to the fact that participants had normal values at start of the supplementation period for most parameters. Furthermore, other dietary carotenoids may have masked the effects of the bacterial carotenoids, or the production and/or absorption of the bacterial carotenoids was not high enough to induce systemic health effects.

In summary, the use of bacterial carotenoids over plant-based carotenoids may be preferred for several reasons. In vitro studies have shown that these bacterial carotenoids deliver gastric-stable carotenoids, which have equal or larger bioaccessibility and bioavailability levels than other, well-established sources of dietary carotenoids, and exert a higher antioxidant capacity. Furthermore, the carotenoid-producing bacillus strain also possesses the ability to act as a probiotic product. ${ }^{204}$ In addition, a microbial fermentation using standard optimized conditions allows for producing a high-quality product with a consistent purity. However, more human in vivo studies are required to confirm the improved bioavailability, antioxidant capacity and systemic health effects of bacterial carotenoids over plant-based carotenoids.

\section{Implications, suggestions for future research}

Food provides taste, aroma, nutritive value and the elements for an individual to grow and live. Recently, functional and bioactive components from foods, inducing positive health effects, have been identified, the so-called functional foods. In this thesis, we have described the results of six randomized controlled trials investigating different nutritional interventions in healthy, overweight and obese individuals. All of the studied nutritional interventions may affect one or more functions in the body in a positive way and thus may be considered a functional food.

In summary, we examined food-drug interactions on the intestinal absorption of a compound, using paracetamol. In addition, the in vivo bioavailability of a polyphenol and of bacterial carotenoids was explored. The efficacy of a prolyl endoprotease in gastrointestinal gluten degradation was investigated in healthy humans. In overweight and obese individuals, the gut microbiota and gut barrier modulating capacities of a potential pre- and probiotic product were assessed. Furthermore, the effects of a polyphenol (hesperidin) and of bacterial carotenoids on cardiovascular and metabolic health parameters have been studied.

The potential of the gluten-degrading enzyme AN-PEP is obvious; it efficiently degrades gluten from a meal, within the time period a meal usually resides in the stomach. From a clinical perspective, additional data are needed from randomized controlled trials, 
exploring the efficacy of AN-PEP in an actual meal setting in subjects intolerant to gluten. Targeting the gut microbiota by pre- and probiotics seems to be a promising approach to prevent the development or to ameliorate many gastrointestinal and metabolic diseases. In vitro and animal studies are widely available, showing convincing and reproducible data of pre- and probiotic products on gastrointestinal and metabolic health. However, human data are scarce. In this thesis we found clear AX-related effects on gut microbial activity, limited AX-related effects on the gut barrier and immune system, and no effects regarding gut microbiota composition. The putative probiotic, which produces carotenoids, did not induce any effects on the gut microbiota composition and function or on gut barrier, which might be considered positive: stimulating a metabolic effect (carotenoid production) without further affecting microbiota composition or intestinal homeostasis. In this thesis we also studied the effects of two different phytochemicals in overweight and obese individuals. Both hesperidin and carotenoids act as antioxidants, and many in vitro and animal studies have reported consistent, beneficial effects of these compounds on cardiovascular and metabolic parameters. Again, data in humans are contradictory. In our human studies, hesperidin showed to have beneficial influences endothelial function and blood pressure, while the carotenoids did not induce significant improvements. In order to exert systemic health effects it is essential that a compound is bioavailable and absorbed from the gastrointestinal tract into the systemic circulation. We have observed that several factors affect bioavailability and absorption of a compound including structure, solubility and particle size of the compound, gut microbiota composition and activity, and bioaccessibility. The different compounds, studied in this thesis, all showed to have beneficial effects in in vitro and in animal studies, but their efficacy in humans remains equivocal. Several explanations can be given for this.

In most studies we investigated the effects of the nutritional intervention in overweight and obese individuals. We consider overweight and obesity as "mild disease", associated with a broad spectrum of well-recognized risk factors for the development of chronic metabolic diseases such as cardiovascular diseases and diabetes mellitus type 2. However, usually these individuals were healthy at baseline. Therefore, an improvement of an already 'stable and excellent' onset condition is not to be anticipated after giving an intervention. Furthermore, a great variation exists between individuals with regard to general health, genetic make-up and expression, gut microbiome, environmental factors, lifestyle and diet. Also, large inter-individual variability exists regarding metabolite excretion of the compounds that have been described. ${ }^{140,205}$ In addition, components of the habitual diet can impact on the bioavailability of a compound. We could not control these factors in our studies. This leads to large inter-individual differences regarding the bioavailability, absorption and biological activity of a compound.

The development of nutritional therapies that target on either prevention or on improvement of an already existing derangement of chronic gastrointestinal, cardiovascular and 
metabolic diseases is important and is considered an attractive alternative for medication use. First, we need large, well-designed, placebo-controlled randomized trials investigating the safety and efficacy in well-defined or phenotyped high-risk populations. Another approach is to apply a stressor in order to induce a disturbance in homeostasis and evaluate the nutritional intervention either before or during application of the stressor. It is important that the international scientific community reaches consensus on standardized study designs for nutritional interventions in human beings. In addition, it is mandatory to study nutritional interventions in well-defined study populations to clarify in which people the intervention is actually effective. 


\section{References}

1. Bellisle F, Blundell JE, Dye L, et al. Functional food science and behaviour and psychological functions. The British journal of nutrition. 1998;80 Suppl 1:S173-93.

2. Clydesdale FM. A proposal for the establishment of scientific criteria for health claims for functional foods. Nutrition reviews. 1997;55(12):413-22.

3. Nations FaAOotU. The state of food insecurity in the world 20152015 [Available from: http://www.fao.org/hunger/en/.

4. Organization WH. Global health risks: mortality and burden of disease attributable to selected major risks. 2009 [Available from:

http://www.who.int/healthinfo/global_burden_disease/GlobalHealthRisks_report_full.pdf.

5. Organization WH. Obesity and overweight. Fact sheet No. 211. http://www.who.int/mediacentre/ factsheets/fs 31 1/en/: World Health Organization Media Centre; 2015

6. Ley RE. Obesity and the human microbiome. Current opinion in gastroenterology. 2010;26(1):5- 11 .

7. Cani PD, Neyrinck AM, Fava F, et al. Selective increases of bifidobacteria in gut microflora improve highfat-diet-induced diabetes in mice through a mechanism associated with endotoxaemia. Diabetologia. 2007:50( 1 1):2374-83.

8. Sekirov I, Russell SL, Antunes LC, et al. Gut microbiota in health and disease. Physiological reviews. 2010;90(3):859-904.

9. Cani PD, Possemiers S, Van de Wiele T, et al. Changes in gut microbiota control inflammation in obese mice through a mechanism involving GLP-2-driven improvement of gut permeability. Gut. 2009;58(8): 1091-103.

10. Turner JR. Intestinal mucosal barrier function in health and disease. Nature reviews Immunology. 2009;9(1 1):799-809.

11. Cani PD, Amar J, Iglesias MA, et al. Metabolic endotoxemia initiates obesity and insulin resistance. Diabetes. 2007;56(7): 176 1-72

12. Cani PD, Bibiloni R, Knauf $C$, et al. Changes in gut microbiota control metabolic endotoxemia-induced inflammation in high-fat diet-induced obesity and diabetes in mice. Diabetes. 2008;57(6): 1470-81.

13. Hotamisligil GS. Inflammation and metabolic disorders. Nature. 2006;444(7121):860-7.

14. de La Serre CB, Ellis CL, Lee J, et al. Propensity to high-fat diet-induced obesity in rats is associated with changes in the gut microbiota and gut inflammation. American journal of physiology Gastrointestinal and liver physiology. 2010;299(2):G440-8.

15. Campia U, Tesauro M, Cardillo C. Human obesity and endothelium-dependent responsiveness. British journal of pharmacology. 2012;165(3):561-73

16. Kang YS. Obesity associated hypertension: new insights into mechanism. Electrolyte \& blood pressure : E \& BP. 2013; 11 (2):46-52.

17. Kahn SE, Hull RL, Utzschneider KM. Mechanisms linking obesity to insulin resistance and type 2 diabetes. Nature. 2006;444(7121):840-6.

18. Franssen R, Monajemi H, Stroes ES, et al. Obesity and dyslipidemia. The Medical clinics of North America. 201 1:95(5):893-902.

19. Van Gaal LF, Mertens IL, De Block CE. Mechanisms linking obesity with cardiovascular disease. Nature. 2006;444(7121):875-80.

20. Wilson PW, D'Agostino RB, Parise H, et al. Metabolic syndrome as a precursor of cardiovascular disease and type 2 diabetes mellitus. Circulation. 2005; 11 2(20):3066-72.

21. Gunderson EP, Lewis CE, Tsai AL, et al. A 20-year prospective study of childbearing and incidence of diabetes in young women, controlling for glycemia before conception: the Coronary Artery Risk Development in Young Adults (CARDIA) Study. Diabetes. 2007;56(12):2990-6. 
22. Organization WH. Obesity: preventing and managing the global epidemic. Report of a WHO Consultation. WHO Technical Report Series, No. 894: World Health Organization; 2000 [Available from: http://www.who.int/nutrition/publications/obesity/en/index.html.

23. Catassi C, Bai JC, Bonaz B, et al. Non-Celiac Gluten sensitivity: the new frontier of gluten related disorders. Nutrients. 2013;5(10):3839-53.

24. Aziz I, Dwivedi K, Sanders DS. From coeliac disease to noncoeliac gluten sensitivity; should everyone be gluten free? Current opinion in gastroenterology. 2016;32(2): 120-7.

25. Green PH, Cellier C. Celiac disease. The New England journal of medicine. 2007;357(17): 1731-43.

26. Sapone A, Lammers KM, Mazzarella G, et al. Differential mucosal IL-17 expression in two gliadin-induced disorders: gluten sensitivity and the autoimmune enteropathy celiac disease. International archives of allergy and immunology. 2010; 152(1):75-80.

27. Sapone A, Lammers KM, Casolaro $V$, et al. Divergence of gut permeability and mucosal immune gene expression in two gluten-associated conditions: celiac disease and gluten sensitivity. BMC medicine. 201 1;9:23.

28. Lee AR, $\mathrm{Ng} \mathrm{DL}$, Zivin J, et al. Economic burden of a gluten-free diet. Journal of human nutrition and dietetics: the official journal of the British Dietetic Association. 2007;20(5):423-30

29. Collin P, Thorell L, Kaukinen K, et al. The safe threshold for gluten contamination in gluten-free products. Can trace amounts be accepted in the treatment of coeliac disease? Alimentary pharmacology \& therapeutics. 2004; 19(12): 1277-83.

30. Wild D, Robins GG, Burley VJ, et al. Evidence of high sugar intake, and low fibre and mineral intake, in the gluten-free diet. Alimentary pharmacology \& therapeutics. 2010;32(4):573-81.

31. Ciacci C, Maiuri L, Caporaso N, et al. Celiac disease: in vitro and in vivo safety and palatability of wheatfree sorghum food products. Clinical nutrition. 2007;26(6):799-805.

32. Spaenii-Dekking L, Kooy-Winkelaar $Y$, van Veelen $P$, et al. Natural variation in toxicity of wheat: potential for selection of nontoxic varieties for celiac disease patients. Gastroenterology. 2005; 129(3):797-806.

33. Gianfrani C, Siciliano RA, Facchiano AM, et al. Transamidation of wheat flour inhibits the response to gliadin of intestinal T cells in celiac disease. Gastroenterology. 2007; 133(3):780-9.

34. Di Cagno R, Barbato M, Di Camillo C, et al. Gluten-free sourdough wheat baked goods appear safe for young celiac patients: a pilot study. Journal of pediatric gastroenterology and nutrition. 2010;51(6):777-83.

35. Di Cagno R, De Angelis M, Auricchio $S$, et al. Sourdough bread made from wheat and nontoxic flours and started with selected lactobacilli is tolerated in celiac sprue patients. Applied and environmental microbiology. 2004;70(2): 1088-96.

36. Greco L, Gobbetti M, Auricchio R, et al. Safety for patients with celiac disease of baked goods made of wheat flour hydrolyzed during food processing. Clin Gastroenterol Hepatol. 201 1; 9(1):24-9.

37. Pinier M, Fuhrmann G, Galipeau HJ, et al. The copolymer P(HEMA-co-SS) binds gluten and reduces immune response in gluten-sensitized mice and human tissues. Gastroenterology. 2012; 142(2):316-25 e 1- 12.

38. Shan L, Molberg $\bigcirc$, Parrot I, et al. Structural basis for gluten intolerance in celiac sprue. Science. 2002;297(5590):2275-9.

39. Kaukinen K, Lindfors K. Novel treatments for celiac disease: glutenases and beyond. Digestive diseases. 2015;33(2):277-81.

40. Stepniak D, Spaenii-Dekking L, Mitea C, et al. Highly efficient gluten degradation with a newly identified prolyl endoprotease: implications for celiac disease. American journal of physiology Gastrointestinal and liver physiology. 2006;291(4):G621-9.

41. Mitea C, Havenaar R, Drijfhout JW, et al. Efficient degradation of gluten by a prolyl endoprotease in a gastrointestinal model: implications for coeliac disease. Gut. 2008;57( 1):25-32.

42. Gass J, Vora H, Bethune MT, et al. Effect of barley endoprotease EP-B2 on gluten digestion in the intact rat. The Journal of pharmacology and experimental therapeutics. 2006;318(3):1 178-86.

43. Gass J, Bethune MT, Siegel M, et al. Combination enzyme therapy for gastric digestion of dietary gluten in patients with celiac sprue. Gastroenterology. 2007; 133(2):472-80. 
44. Matysiak-Budnik T, Candalh C, Cellier C, et al. Limited efficiency of prolyl-endopeptidase in the detoxification of gliadin peptides in celiac disease. Gastroenterology. 2005; 129(3):786-96.

45. Siegel M, Garber ME, Spencer AG, et al. Safety, tolerability, and activity of ALVOO3: results from two phase 1 single, escalating-dose clinical trials. Digestive diseases and sciences. 2012;57(2):440-50.

46. Lahdeaho ML, Kaukinen K, Laurila K, et al. Glutenase ALVOO3 attenuates gluten-induced mucosal injury in patients with celiac disease. Gastroenterology. 2014; 146(7): 1649-58.

47. Bethune MT, Ribka E, Khosla C, et al. Transepithelial transport and enzymatic detoxification of gluten in gluten-sensitive rhesus macaques. PloS one. 2008;3(3):e1857.

48. Hunt JN. A possible relation between the regulation of gastric emptying and food intake. The American journal of physiology. 1980;239(1):G1-4.

49. Welling PG. Effects of food on drug absorption. Annual review of nutrition. 1996; 16:383-415.

50. Sensoy I. A review on the relationship between food structure, processing, and bioavailability. Critical reviews in food science and nutrition. 2014;54(7):902-9.

51. Baquero F, Nombela C. The microbiome as a human organ. Clinical microbiology and infection : the official publication of the European Society of Clinical Microbiology and Infectious Diseases. 2012;18 Suppl 4:2-4.

52. Backhed F, Ding H, Wang $T$, et al. The gut microbiota as an environmental factor that regulates fat storage. Proceedings of the National Academy of Sciences of the United States of America. 2004; 101(44): 15718-23.

53. Nieuwdorp M, Gilijamse PW, Pai N, et al. Role of the microbiome in energy regulation and metabolism. Gastroenterology. 2014; 146(6): 1525-33.

54. Turnbaugh PJ, Ley RE, Mahowald MA, et al. An obesity-associated gut microbiome with increased capacity for energy harvest. Nature. 2006;444(7122):1027-31.

55. Vandeputte D, Falony G, Vieira-Silva $S$, et al. Stool consistency is strongly associated with gut microbiota richness and composition, enterotypes and bacterial growth rates. Gut. 2016;65(1):57-62.

56. Chan YK, Estaki M, Gibson DL. Clinical consequences of diet-induced dysbiosis. Annals of nutrition \& metabolism. 2013:63 Suppl 2:28-40.

57. Nagao-Kitamoto $\mathrm{H}$, Kitamoto $\mathrm{S}, \mathrm{Kuffa} \mathrm{P}$, et al. Pathogenic role of the gut microbiota in gastrointestinal diseases. Intestinal research. 2016; 14(2): 127-38.

58. McLoughlin RM, Mills KH. Influence of gastrointestinal commensal bacteria on the immune responses that mediate allergy and asthma. The Journal of allergy and clinical immunology. 2011;127(5):1097-107; quiz 108-9.

59. Abu-Shanab A, Quigley EM. The role of the gut microbiota in nonalcoholic fatty liver disease. Nature reviews Gastroenterology \& hepatology. 2010;7(12):691-701.

60. Okeke F, Roland BC, Mullin GE. The role of the gut microbiome in the pathogenesis and treatment of obesity. Global advances in health and medicine: improving healthcare outcomes worldwide. 2014;3(3): 44-57.

61. Turnbaugh PJ, Hamady M, Yatsunenko T, et al. A core gut microbiome in obese and lean twins. Nature. 2009:457(7228):480-4

62. Wen L, Ley RE, Volchkov PY, et al. Innate immunity and intestinal microbiota in the development of Type 1 diabetes. Nature. 2008;455(7216): 1 109-13

63. Gibson GR, Roberfroid MB. Dietary modulation of the human colonic microbiota: introducing the concept of prebiotics. The Journal of nutrition. 1995; 125(6):1401-12.

64. Marchesi JR, Adams DH, Fava F, et al. The gut microbiota and host health: a new clinical frontier. Gut. 2016;65(2):330-9.

65. Canani RB, Costanzo MD, Leone L, et al. Potential beneficial effects of butyrate in intestinal and extraintestinal diseases. World journal of gastroenterology. 201 1; 17(12): 1519-28.

66. Zhong Y, Teixeira C, Marungruang N, et al. Barley malt increases hindgut and portal butyric acid, modulates gene expression of gut tight junction proteins and Toll-like receptors in rats fed high-fat diets, but high advanced glycation end-products partially attenuate the effects. Food \& function. 2015;6(9):3165-76. 
67. Peng L, Li ZR, Green RS, et al. Butyrate enhances the intestinal barrier by facilitating tight junction assembly via activation of AMP-activated protein kinase in Caco-2 cell monolayers. The Journal of nutrition. 2009; 139(9): $1619-25$.

68. Topping DL, Fukushima M, Bird AR. Resistant starch as a prebiotic and synbiotic: state of the art. The Proceedings of the Nutrition Society. 2003;62(1):171-6.

69. den Besten G, van Eunen K, Groen AK, et al. The role of short-chain fatty acids in the interplay between diet, gut microbiota, and host energy metabolism. Journal of lipid research. 2013;54(9):2325-40.

70. Van den Abbeele P, Gerard P, Rabot S, et al. Arabinoxylans and inulin differentially modulate the mucosal and luminal gut microbiota and mucin-degradation in humanized rats. Environmental microbiology. $2011 ; 13(10): 2667-80$

71. Kolida S, Tuohy K, Gibson GR. Prebiotic effects of inulin and oligofructose. Brit J Nutr. 2002;87:S193-S7.

72. Hughes SA, Shewry PR, Li L, et al. In vitro fermentation by human fecal microflora of wheat arabinoxylans. Journal of agricultural and food chemistry. 2007;55(1 1):4589-95.

73. Vardakou M, Palop CN, Christakopoulos $P$, et al. Evaluation of the prebiotic properties of wheat arabinoxylan fractions and induction of hydrolase activity in gut microflora. Int J Food Microbiol. 2008; 123(1-2): 166-70.

74. Grootaert C, Van den Abbeele P, Marzorati M, et al. Comparison of prebiotic effects of arabinoxylan oligosaccharides and inulin in a simulator of the human intestinal microbial ecosystem. FEMS microbiology ecology. 2009;69(2):23 1-42.

75. Neyrinck AM, Possemiers S, Druart C, et al. Prebiotic effects of wheat arabinoxylan related to the increase in bifidobacteria, Roseburia and Bacteroides/Prevotella in diet-induced obese mice. PloS one. $2011 ; 6(6): e 20944$.

76. Chen H, Wang W, Degroote J, et al. Arabinoxylan in wheat is more responsible than cellulose for promoting intestinal barrier function in weaned male piglets. The Journal of nutrition. 2015; 145(1):51-8.

77. Van den Abbeele P, Venema K, Van de Wiele T, et al. Different human gut models reveal the distinct fermentation patterns of Arabinoxylan versus inulin. Journal of agricultural and food chemistry. 2013:61(41):9819-27.

78. Kleessen B, Hartmann L, Blaut M. Fructans in the diet cause alterations of intestinal mucosal architecture, released mucins and mucosa-associated bifidobacteria in gnotobiotic rats. The British journal of nutrition. 2003;89(5):597-606

79. Muccioli GG, Naslain D, Backhed F, et al. The endocannabinoid system links gut microbiota to adipogenesis. Molecular systems biology. 2010;6:392.

80. Toden S, Bird AR, Topping DL, et al. Dose-dependent reduction of dietary protein-induced colonocyte DNA damage by resistant starch in rats correlates more highly with caecal butyrate than with other short chain fatty acids. Cancer biology \& therapy. 2007;6(2):253-8.

81. Mariadason JM, Barkla DH, Gibson PR. Effect of short-chain fatty acids on paracellular permeability in Caco-2 intestinal epithelium model. The American journal of physiology. 1997;272(4 Pt 1):G705- 12

82. Hartsock A, Nelson WJ. Adherens and tight junctions: structure, function and connections to the actin cytoskeleton. Biochimica et biophysica acta. 2008; 1778(3):660-9.

83. Anderson JM, Van Itallie CM. Physiology and function of the tight junction. Cold Spring Harbor perspectives in biology. 2009; 1(2):a002584.

84. Peterson CT, Sharma V, Elmen L, et al. Immune homeostasis, dysbiosis and therapeutic modulation of the gut microbiota. Clinical and experimental immunology. 2015; 179(3):363-77.

85. Capitan-Canadas F, Ortega-Gonzalez M, Guadix E, et al. Prebiotic oligosaccharides directly modulate proinflammatory cytokine production in monocytes via activation of TLR4. Molecular nutrition \& food research. 2014;58(5): 1098-110.

86. Ortega-Gonzalez M, Ocon B, Romero-Calvo I, et al. Nondigestible oligosaccharides exert nonprebiotic effects on intestinal epithelial cells enhancing the immune response via activation of TLR4-NFkappaB. Molecular nutrition \& food research. 2014;58(2):384-93. 
87. Macfarlane GT, Steed H, Macfarlane S. Bacterial metabolism and health-related effects of galactooligosaccharides and other prebiotics. Journal of applied microbiology. 2008; 104(2):305-44.

88. Boehm G, Stahl B, Jelinek J, et al. Prebiotic carbohydrates in human milk and formulas. Acta paediatrica. 2005;94(449): 18-21.

89. Langlands SJ, Hopkins MJ, Coleman N, et al. Prebiotic carbohydrates modify the mucosa associated microflora of the human large bowel. Gut. 2004;53(1 1):1610-6.

90. Gibson GR, Wang X. Enrichment of bifidobacteria from human gut contents by oligofructose using continuous culture. FEMS microbiology letters. 1994; 1 18(1-2):121-7.

91. Pratt VC, Tappenden KA, McBurney MI, et al. Short-chain fatty acid-supplemented total parenteral nutrition improves nonspecific immunity after intestinal resection in rats. JPEN Journal of parenteral and enteral nutrition. 1996;20(4):264-71.

92. Bohmig GA, Krieger PM, Saemann MD, et al. n-butyrate downregulates the stimulatory function of peripheral blood-derived antigen-presenting cells: a potential mechanism for modulating T-cell responses by short-chain fatty acids. Immunology. 1997;92(2):234-43

93. Vinolo MA, Rodrigues HG, Nachbar RT, et al. Regulation of inflammation by short chain fatty acids. Nutrients. $2011 ; 3(10): 858-76$

94. Caselli M, Cassol F, Calo G, et al. Actual concept of "probiotics": is it more functional to science or business? World J Gastroenterol. 2013; 19(10): 1527-40

95. Penner R, Fedorak RN, Madsen KL. Probiotics and nutraceuticals: non-medicinal treatments of gastrointestinal diseases. Curr Opin Pharmacol. 2005;5(6):596-603

96. Nagpal R, Kumar A, Kumar M, et al. Probiotics, their health benefits and applications for developing healthier foods: a review. FEMS microbiology letters. 2012;334(1): 1-15

97. Nicholson WL. Roles of Bacillus endospores in the environment. Cellular and molecular life sciences: CMLS. 2002;59(3):410-6.

98. Hong HA, Duc le H, Cutting SM. The use of bacterial spore formers as probiotics. FEMS microbiology reviews. 2005;29(4):813-35

99. Quigley EM. From comic relief to real understanding; how intestinal gas causes symptoms. Gut. 2003;52(12): 1659-61.

100. Johansson ML, Molin G, Jeppsson B, et al. Administration of different Lactobacillus strains in fermented oatmeal soup: in vivo colonization of human intestinal mucosa and effect on the indigenous flora. Applied and environmental microbiology. 1993;59(1):15-20.

101. Nobaek S, Johansson ML, Molin G, et al. Alteration of intestinal microflora is associated with reduction in abdominal bloating and pain in patients with irritable bowel syndrome. The American journal of gastroenterology. 2000;95(5): 1231-8.

102. Kalman DS, Schwartz HI, Alvarez P, et al. A prospective, randomized, double-blind, placebo-controlled parallel-group dual site trial to evaluate the effects of a Bacillus coagulans-based product on functional intestinal gas symptoms. BMC gastroenterology. 2009:9:85.

103. Cappello C, Tremolaterra F, Pascariello A, et al. A randomised clinical trial (RCT) of a symbiotic mixture in patients with irritable bowel syndrome (IBS): effects on symptoms, colonic transit and quality of life. International journal of colorectal disease. 2013;28(3):349-58.

104. Silk DB, Davis A, Vulevic J, et al. Clinical trial: the effects of a trans-galactooligosaccharide prebiotic on faecal microbiota and symptoms in irritable bowel syndrome. Alimentary pharmacology \& therapeutics. 2009;29(5):508-18

105. Brugman S, Klatter FA, Visser JT, et al. Antibiotic treatment partially protects against type 1 diabetes in the Bio-Breeding diabetes-prone rat. Is the gut flora involved in the development of type 1 diabetes? Diabetologia. 2006; 49(9):2 105-8

106. Cani PD, Daubioul CA, Reusens B, et al. Involvement of endogenous glucagon-like peptide- 1(7-36) amide on glycaemia-lowering effect of oligofructose in streptozotocin-treated rats. The Journal of endocrinology. 2005; 185(3):457-65 
107. Yadav H, Jain S, Sinha PR. Antidiabetic effect of probiotic dahi containing Lactobacillus acidophilus and Lactobacillus casei in high fructose fed rats. Nutrition. 2007;23( 1):62-8.

108. Grover GJ, Koetzner L, Wicks J, et al. Effects of the Soluble Fiber Complex PolyGlycopleX on Glucose Homeostasis and Body Weight in Young Zucker Diabetic Rats. Frontiers in pharmacology. 201 1;2:47.

109. Velasco S, Ortiz LT, Alzueta C, et al. Effect of inulin supplementation and dietary fat source on performance, blood serum metabolites, liver lipids, abdominal fat deposition, and tissue fatty acid composition in broiler chickens. Poultry science. 2010;89(8): 1651-62.

1 10. Le TK, Hosaka T, Nguyen TT, et al. Bifidobacterium species lower serum glucose, increase expressions of insulin signaling proteins, and improve adipokine profile in diabetic mice. Biomedical research. 2015;36(1):63-70.

1 11. Russo F, Linsalata M, Clemente $C$, et al. Inulin-enriched pasta improves intestinal permeability and modifies the circulating levels of zonulin and glucagon-like peptide 2 in healthy young volunteers. Nutrition research. 2012;32(12):940-6.

112. Dewulf EM, Cani PD, Claus SP, et al. Insight into the prebiotic concept: lessons from an exploratory, double blind intervention study with inulin-type fructans in obese women. Gut. 2013;62(8): $1112-21$.

113. Causey JL, Feirtag JM, Gallaher DD, et al. Effects of dietary inulin on serum lipids, blood glucose and the gastrointestinal, environment in hypercholesterolemic men. Nutrition research. 2000;20(2): 191-201.

1 14. Giacco R, Clemente G, Luongo D, et al. Effects of short-chain fructo-oligosaccharides on glucose and lipid metabolism in mild hypercholesterolaemic individuals. Clinical nutrition. 2004;23(3):331-40.

1 15. Moroti C, Magri LFS, Costa MD, et al. Effect of the consumption of a new symbiotic shake on glycemia and cholesterol levels in elderly people with type 2 diabetes mellitus. Lipids Health Dis. 2012; 11.

1 16. Brahe LK, Le Chatelier E, Prifti E, et al. Dietary modulation of the gut microbiota--a randomised controlled trial in obese postmenopausal women. The British journal of nutrition. 2015; 1 14(3):406-17.

117. Madid A, Taylor MA, Mousavi N, et al. Comparison of the effect of daily consumption of probiotic compared with low-fat conventional yogurt on weight loss in healthy obese women following an energyrestricted diet: a randomized controlled trial. The American journal of clinical nutrition. 2016; 103(2):323-9.

1 18. Fuentes MC, Lajo T, Carrion JM, et al. Cholesterol-lowering efficacy of Lactobacillus plantarum CECT 7527, 7528 and 7529 in hypercholesterolaemic adults. The British journal of nutrition. 2013; 109(10): 1866-72.

1 19. Lewis SJ, Burmeister S. A double-blind placebo-controlled study of the effects of Lactobacillus acidophilus on plasma lipids. European journal of clinical nutrition. 2005;59(6):776-80.

120. Bazzano LA, He J, Ogden LG, et al. Fruit and vegetable intake and risk of cardiovascular disease in US adults: the first National Health and Nutrition Examination Survey Epidemiologic Follow-up Study. The American journal of clinical nutrition. 2002;76(1):93-9.

121. Mursu J, Voutilainen S, Nurmi T, et al. Flavonoid intake and the risk of ischaemic stroke and CVD mortality in middle-aged Finnish men: the Kuopio Ischaemic Heart Disease Risk Factor Study. The British journal of nutrition. 2008; 100(4):890-5.

122. Hooper L, Kroon PA, Rimm EB, et al. Flavonoids, flavonoid-rich foods, and cardiovascular risk: a metaanalysis of randomized controlled trials. The American journal of clinical nutrition. 2008;88(1):38-50.

123. Cassidy A, Rimm EB, O'Reilly EJ, et al. Dietary flavonoids and risk of stroke in women. Stroke; a journal of cerebral circulation. 2012;43(4):946-51.

124. Takumi $H$, Nakamura $H$, Simizu T, et al. Bioavailability of orally administered water-dispersible hesperetin and its effect on peripheral vasodilatation in human subjects: implication of endothelial functions of plasma conjugated metabolites. Food \& function. 2012;3(4):389-98.

125. Morand C, Dubray C, Milenkovic D, et al. Hesperidin contributes to the vascular protective effects of orange juice: a randomized crossover study in healthy volunteers. The American journal of clinical nutrition. 2011 1:93(1):73-80.

126. Rizza S, Muniyappa R, lantorno M, et al. Citrus polyphenol hesperidin stimulates production of nitric oxide in endothelial cells while improving endothelial function and reducing inflammatory markers in patients with metabolic syndrome. The Journal of clinical endocrinology and metabolism. 201 1;96(5):E782-92. 
127. Haidari F, Heybar H, Jalali MT, et al. Hesperidin supplementation modulates inflammatory responses following myocardial infarction. Journal of the American College of Nutrition. 2015;34(3):205- 11.

128. Kurowska EM, Spence JD, Jordan J, et al. HDL-cholesterol-raising effect of orange juice in subjects with hypercholesterolemia. The American journal of clinical nutrition. 2000;72(5): 1095-100.

129. Mulvihill EE, Burke AC, Huff MW. Citrus Flavonoids as Regulators of Lipoprotein Metabolism and Atherosclerosis. Annual review of nutrition. 2016.

130. Erlund I, Meririnne E, Alfthan G, et al. Plasma kinetics and urinary excretion of the flavanones naringenin and hesperetin in humans after ingestion of orange juice and grapefruit juice. The Journal of nutrition. 2001; 131(2):235-41.

131. Manach C, Morand C, Gil-Izquierdo A, et al. Bioavailability in humans of the flavanones hesperidin and narirutin after the ingestion of two doses of orange juice. European journal of clinical nutrition. 2003;57(2):235-42.

132. Gil-Izquierdo A, Gil MI, Tomas-Barberan FA, et al. Influence of industrial processing on orange juice flavanone solubility and transformation to chalcones under gastrointestinal conditions. Journal of agricultural and food chemistry. 2003;51(10):3024-8.

133. Amaretti A, Raimondi S, Leonardi A, et al. Hydrolysis of the rutinose-conjugates flavonoids rutin and hesperidin by the gut microbiota and bifidobacteria. Nutrients. 2015;7(4):2788-800

134. Matsumoto $H$, Ikoma $Y$, Sugiura $M$, et al. Identification and quantification of the conjugated metabolites derived from orally administered hesperidin in rat plasma. Journal of agricultural and food chemistry. 2004;52(21):6653-9.

135. Rossi $M$, Amaretti $A$, Leonardi A, et al. Potential impact of probiotic consumption on the bioactivity of dietary phytochemicals. Journal of agricultural and food chemistry. 2013;61(40):9551-8.

136. Bredsdorff L, Nielsen IL, Rasmussen SE, et al. Absorption, conjugation and excretion of the flavanones, naringenin and hesperetin from alpha-rhamnosidase-treated orange juice in human subjects. The British journal of nutrition. 2010; 103(1 1):1602-9.

137. Nielsen IL, Chee WS, Poulsen L, et al. Bioavailability is improved by enzymatic modification of the citrus flavonoid hesperidin in humans: a randomized, double-blind, crossover trial. The Journal of nutrition. 2006; 136(2):404-8.

138. Gonzalez-Barrio R, Trindade LM, Manzanares P, et al. Production of bioavailable flavonoid glucosides in fruit juices and green tea by use of fungal alpha-L-rhamnosidases. Journal of agricultural and food chemistry. 2004;52(20):6136-42.

139. Chaumeil JC. Micronization: a method of improving the bioavailability of poorly soluble drugs. Methods and findings in experimental and clinical pharmacology. 1998;20(3):21 1-5.

140. Cerda B, Tomas-Barberan FA, Espin JC. Metabolism of antioxidant and chemopreventive ellagitannins from strawberries, raspberries, walnuts, and oak-aged wine in humans: identification of biomarkers and individual variability. Journal of agricultural and food chemistry. 2005;53(2):227-35

141. Lee MJ, Maliakal P, Chen L, et al. Pharmacokinetics of tea catechins after ingestion of green tea and (-)epigallocatechin-3-gallate by humans: formation of different metabolites and individual variability. Cancer epidemiology, biomarkers \& prevention : a publication of the American Association for Cancer Research, cosponsored by the American Society of Preventive Oncology. 2002; 1 1(10 Pt 1): 1025-32.

142. Del Rio D, Rodriguez-Mateos A, Spencer JP, et al. Dietary (poly)phenolics in human health: structures, bioavailability, and evidence of protective effects against chronic diseases. Antioxidants \& redox signaling. 2013; 18(14): 1818-92.

143. Rowland IR, Wiseman H, Sanders TA, et al. Interindividual variation in metabolism of soy isoflavones and lignans: influence of habitual diet on equol production by the gut microflora. Nutrition and cancer. 2000;36( 1):27-32.

144. Bode $L M$, Bunzel $D$, Huch $M$, et al. In vivo and in vitro metabolism of trans-resveratrol by human gut microbiota. The American journal of clinical nutrition. 2013;97(2):295-309. 
145. Wilmsen PK, Spada DS, Salvador M. Antioxidant activity of the flavonoid hesperidin in chemical and biological systems. Journal of agricultural and food chemistry. 2005;53(12):4757-61.

146. Hirata A, Murakami Y, Shoii M, et al. Kinetics of radical-scavenging activity of hesperetin and hesperidin and their inhibitory activity on COX-2 expression. Anticancer research. 2005;25(5):3367-74.

147. Napoli C, Ignarro LJ. Nitric oxide and atherosclerosis. Nitric oxide : biology and chemistry / official journal of the Nitric Oxide Society. 2001;5(2):88-97.

148. Rees DD, Palmer RM, Moncada S. Role of endothelium-derived nitric oxide in the regulation of blood pressure. Proceedings of the National Academy of Sciences of the United States of America. 1989;86(9):3375-8.

149. Huang PL, Huang Z, Mashimo H, et al. Hypertension in mice lacking the gene for endothelial nitric oxide synthase. Nature. 1995;377(6546):239-42.

150. Mudau M, Genis A, Lochner A, et al. Endothelial dysfunction: the early predictor of atherosclerosis. Cardiovascular journal of Africa. 2012;23(4):222-31.

151. Yamamoto M, Suzuki A, Hase T. Short-term effects of glucosyl hesperidin and hesperetin on blood pressure and vascular endothelial function in spontaneously hypertensive rats. Journal of nutritional science and vitaminology. 2008;54(1):95-8.

152. Yamamoto M, Suzuki A, Jokura H, et al. Glucosyl hesperidin prevents endothelial dysfunction and oxidative stress in spontaneously hypertensive rats. Nutrition. 2008;24(5):470-6.

153. Sankatsing RR, de Groot E, Jukema JW, et al. Surrogate markers for atherosclerotic disease. Curr Opin Lipidol. 2005; 16(4):434-41.

154. Frolow M, Drozdz A, Kowalewska A, et al. Comprehensive assessment of vascular health in patients; towards endothelium-guided therapy. Pharmacol Rep. 2015;67(4):786-92.

155. Witte DR, Westerink J, de Koning EJ, et al. Is the association between flow-mediated dilation and cardiovascular risk limited to low-risk populations? Journal of the American College of Cardiology. 2005;45(12): 1987-93.

156. Donald AE, Halcox JP, Charakida M, et al. Methodological approaches to optimize reproducibility and power in clinical studies of flow-mediated dilation. Journal of the American College of Cardiology. 2008;51(20): 1959-64.

157. Benetos A, Waeber B, Izzo J, et al. Influence of age, risk factors, and cardiovascular and renal disease on arterial stiffness: clinical applications. American journal of hypertension. 2002; 15(12): 1101 1-8.

158. Amiot MJ, Riva C, Vinet A. Effects of dietary polyphenols on metabolic syndrome features in humans: a systematic review. Obesity reviews : an official journal of the International Association for the Study of Obesity. 2016; 17(7):573-86.

159. Jung UJ, Lee MK, Park YB, et al. Effect of citrus flavonoids on lipid metabolism and glucose-regulating enzyme mRNA levels in type-2 diabetic mice. The international journal of biochemistry \& cell biology. 2006;38(7): 11 134-45

160. Bok SH, Lee SH, Park YB, et al. Plasma and hepatic cholesterol and hepatic activities of 3-hydroxy-3-methylglutaryl-CoA reductase and acyl CoA: cholesterol transferase are lower in rats fed citrus peel extract or a mixture of citrus bioflavonoids. The Journal of nutrition. 1999; 129(6): 1 182-5.

161. Kim HK, Jeong TS, Lee MK, et al. Lipid-lowering efficacy of hesperetin metabolites in high-cholesterol fed rats. Clin Chim Acta. 2003;327(1-2): 129-37.

162. Ahmed F, Hudeda S, Urooj A. Antihyperglycemic activity of Ficus racemosa bark extract in type 2 diabetic individuals. Journal of diabetes. 201 1;3(4):318-9.

163. Zhang B, Chen T, Chen Z, et al. Synthesis and anti-hyperglycemic activity of hesperidin derivatives. Bioorg Med Chem Lett. 2012;22(23):7194-7.

164. Jung UJ, Lee MK, Jeong KS, et al. The hypoglycemic effects of hesperidin and naringin are partly mediated by hepatic glucose-regulating enzymes in C57BL/KsJ-db/db mice. The Journal of nutrition. 2004; 134(10):2499-503. 
165. Miwa Y, Mitsuzumi H, Sunayama T, et al. Glucosyl hesperidin lowers serum triglyceride level in hypertriglyceridemic subjects through the improvement of very low-density lipoprotein metabolic abnormality. Journal of nutritional science and vitaminology. 2005;51(6):460-70.

166. Queipo-Ortuno MI, Boto-Ordonez M, Murri M, et al. Influence of red wine polyphenols and ethanol on the gut microbiota ecology and biochemical biomarkers. The American journal of clinical nutrition. 2012:95(6): 1323-34

167. Vendrame S, Guglielmetti S, Riso P, et al. Six-week consumption of a wild blueberry powder drink increases bifidobacteria in the human gut. Journal of agricultural and food chemistry. 201 1;59(24):128 15-20.

168. Hidalgo M, Oruna-Concha MJ, Kolida S, et al. Metabolism of anthocyanins by human gut microflora and their influence on gut bacterial growth. Journal of agricultural and food chemistry. 2012;60(15):3882-90.

169. Unno T, Hisada T, Takahashi S. Hesperetin Modifies the Composition of Fecal Microbiota and Increases Cecal Levels of Short-Chain Fatty Acids in Rats. Journal of agricultural and food chemistry. 2015:63(36):7952-7

170. Bolca S, Van de Wiele T, Possemiers S. Gut metabotypes govern health effects of dietary polyphenols. Current opinion in biotechnology. 2013;24(2):220-5.

171. Tzounis X, Rodriguez-Mateos A, Vulevic J, et al. Prebiotic evaluation of cocoa-derived flavanols in healthy humans by using a randomized, controlled, double-blind, crossover intervention study. The American journal of clinical nutrition. 201 1:93(1):62-72.

172. Hamer HM, Jonkers D, Venema K, et al. Review article: the role of butyrate on colonic function. Alimentary pharmacology \& therapeutics. 2008;27(2): 104-19.

173. Serra-Majem L, Roman B, Estruch R. Scientific evidence of interventions using the Mediterranean diet: a systematic review. Nutrition reviews. 2006;64(2 Pt 2):S27-47.

174. Muller-Nordhorn J, Binting S, Roll S, et al. An update on regional variation in cardiovascular mortality within Europe. European heart journal. 2008;29(10):1316-26.

175. Hozawa A, Jacobs DR, Jr., Steffes MW, et al. Relationships of circulating carotenoid concentrations with several markers of inflammation, oxidative stress, and endothelial dysfunction: the Coronary Artery Risk Development in Young Adults (CARDIA)/Young Adult Longitudinal Trends in Antioxidants (YALTA) study. Clinical chemistry. 2007;53(3):447-55.

176. Hozawa A, Jacobs DR, Jr., Steffes MW, et al. Circulating carotenoid concentrations and incident hypertension: the Coronary Artery Risk Development in Young Adults (CARDIA) study. Journal of hypertension. 2009;27(2):237-42.

177. Rao AV, Rao LG. Carotenoids and human health. Pharmacological research : the official journal of the Italian Pharmacological Society. 2007;55(3):207-16

178. Krinsky NI. The antioxidant and biological properties of the carotenoids. Annals of the New York Academy of Sciences. 1998;854:443-7.

179. Stahl W, Sies H. Antioxidant activity of carotenoids. Molecular aspects of medicine. 2003;24(6):345-51.

180. Bast A, Haenen GR, van den Berg R, et al. Antioxidant effects of carotenoids. International journal for vitamin and nutrition research Internationale Zeitschrift fur Vitamin- und Ernahrungsforschung Journal international de vitaminologie et de nutrition. 1998;68(6):399-403.

181. van Breemen RB, Pajkovic N. Multitargeted therapy of cancer by lycopene. Cancer letters. 2008;269(2):339-51

182. Holzapfel NP, Holzapfel BM, Champ S, et al. The potential role of lycopene for the prevention and therapy of prostate cancer: from molecular mechanisms to clinical evidence. International journal of molecular sciences. 2013; 14(7): 14620-46

183. Rice-Evans CA, Sampson J, Bramley PM, et al. Why do we expect carotenoids to be antioxidants in vivo? Free radical research. 1997;26(4):381-98.

184. Paiva SA, Russell RM. Beta-carotene and other carotenoids as antioxidants. Journal of the American College of Nutrition. 1999; 18(5):426-33. 
185. Jewell C, O'Brien NM. Effect of dietary supplementation with carotenoids on xenobiotic metabolizing enzymes in the liver, lung, kidney and small intestine of the rat. The British journal of nutrition. 1999;8 1 (3):235-42.

186. Bertram JS. Carotenoids and gene regulation. Nutrition reviews. 1999;57(6):182-91.

187. Duc le H, Fraser PD, Tam NK, et al. Carotenoids present in halotolerant Bacillus spore formers. FEMS microbiology letters. 2006;255(2):215-24.

188. Perez-Fons L, Steiger S, Khaneja R, et al. Identification and the developmental formation of carotenoid pigments in the yellow/orange Bacillus spore-formers. Biochimica et biophysica acta. 201 1; 181 1(3): 177-85

189. Parker RS. Absorption, metabolism, and transport of carotenoids. FASEB journal : official publication of the Federation of American Societies for Experimental Biology. 1996; 10(5):542-51.

190. Khaneja R, Perez-Fons L, Fakhry S, et al. Carotenoids found in Bacillus. Journal of applied microbiology. 2010; 108(6): 1889-902

191. White WS, Stacewicz-Sapuntzakis M, Erdman JW, Jr., et al. Pharmacokinetics of beta-carotene and canthaxanthin after ingestion of individual and combined doses by human subjects. Journal of the American College of Nutrition. 1994; 13(6):665-71.

192. Kostic D, White WS, Olson JA. Intestinal absorption, serum clearance, and interactions between lutein and beta-carotene when administered to human adults in separate or combined oral doses. The American journal of clinical nutrition. 1995;62(3):604-10

193. van den Berg H. Carotenoid interactions. Nutrition reviews. 1999;57(1): 1-10.

194. van den Berg H. Effect of lutein on beta-carotene absorption and cleavage. International journal for vitamin and nutrition research Internationale Zeitschrift fur Vitamin- und Ernahrungsforschung Journal international de vitaminologie et de nutrition. 1998;68(6):360-5.

195. van den Berg $H$, van Vliet T. Effect of simultaneous, single oral doses of beta-carotene with lutein or lycopene on the beta-carotene and retinyl ester responses in the triacylglycerol-rich lipoprotein fraction of men. The American journal of clinical nutrition. 1998;68(1):82-9.

196. Kim JY, Paik JK, Kim OY, et al. Effects of lycopene supplementation on oxidative stress and markers of endothelial function in healthy men. Atherosclerosis. 201 1:215(1):189-95.

197. Garcia-Alonso FJ, Jorge-Vidal V, Ros G, et al. Effect of consumption of tomato juice enriched with n-3 polyunsaturated fatty acids on the lipid profile, antioxidant biomarker status, and cardiovascular disease risk in healthy women. European journal of nutrition. 2012;51(4):415-24.

198. Agarwal S, Rao AV. Tomato lycopene and low density lipoprotein oxidation: a human dietary intervention study. Lipids. 1998;33(10):981-4.

199. Engelhard YN, Gazer B, Paran E. Natural antioxidants from tomato extract reduce blood pressure in patients with grade-1 hypertension: a double-blind, placebo-controlled pilot study. American heart journal. 2006; $151(1): 100$

200. Blum A, Monir M, Khazim K, et al. Tomato-rich (Mediterranean) diet does not modify inflammatory markers. Clinical and investigative medicine Medecine clinique et experimentale. 2007;30(2):E70-4.

201. Thies F, Masson LF, Rudd A, et al. Effect of a tomato-rich diet on markers of cardiovascular disease risk in moderately overweight, disease-free, middle-aged adults: a randomized controlled trial. The American journal of clinical nutrition. 2012;95(5): 1013-22.

202. Silaste ML, Alfthan G, Aro A, et al. Tomato juice decreases LDL cholesterol levels and increases LDL resistance to oxidation. The British journal of nutrition. 2007;98(6): 1251-8.

203. Ried K, Frank OR, Stocks NP. Dark chocolate or tomato extract for prehypertension: a randomised controlled trial. BMC complementary and alternative medicine. 2009:9:22

204. Hong HA, Huang JM, Khaneja R, et al. The safety of Bacillus subtilis and Bacillus indicus as food probiotics. Journal of applied microbiology. 2008; 105(2):510-20.

205. Cerda B, Espin JC, Parra S, et al. The potent in vitro antioxidant ellagitannins from pomegranate juice are metabolised into bioavailable but poor antioxidant hydroxy-6H-dibenzopyran-6-one derivatives by the colonic microflora of healthy humans. European journal of nutrition. 2004;43(4):205-20. 

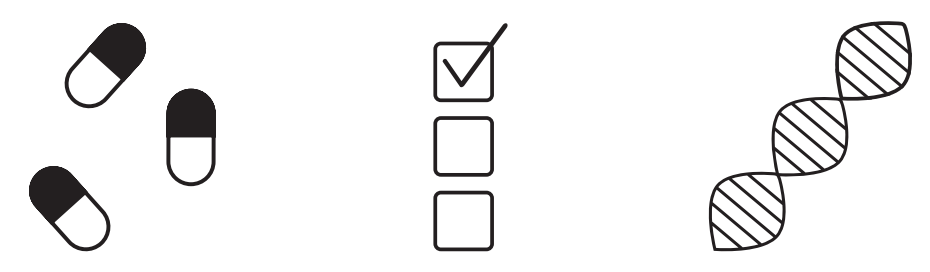
addendum

Summary

Nederlandse samenvatting

Valorisation

List of publications

Curriculum vitae

Dankwoord 


\section{Summary}

The primary role of food is to provide sufficient quantitative and qualitative supply of nutrients to meet the nutritional requirements of an individual. In addition food may also have health promoting effects. Initially, scientific focus was given on the identification of essential elements and their role in the treatment and prevention of various deficiencies and of diseases associated with specific deficiencies. In recent years, the role of diet in diseases linked to excess food intake and to 'overnutrition' has become topic of clinical interest and of research. The discovery and further development of functional foods is a fast growing segment of the food industry. Functional foods are defined as foods or food ingredients that affect one or more functions in the body in a targeted way so that positive effects on health are to be expected, or when the component has a physiological or psychological effect beyond the traditional nutritional effect. The role of functional foods in disease prevention and health promotion is nowadays a research topic.

In this thesis, we aimed to investigate the effects of several nutritional interventions on gastrointestinal and metabolic health in healthy, overweight and obese individuals. The nutritional interventions we studied include a prolyl endoprotease, a putative prebiotic product, a putative probiotic species and two different phytonutriets.

Gluten, a protein rich in proline, is poorly degraded in the gastrointestinal tract due to the gastrointestinal absence of proline-cleaving proteases. The presence of long proline-rich gluten peptides in the small intestine can induce a wide range of gluten related disorders with different pathogenic pathways. Prolyl endoproteases are enzymes that possess the ability to cleave at internal proline residues within the gluten. In Chapter 2 we investigated the in vivo efficacy of an Aspergillus Niger-derived prolyl endoprotease (AN-PEP) in degrading gluten. AN-PEP efficiently degraded gluten from a meal in the stomach of healthy volunteers before entering the duodenum. Furthermore, increasing meal caloric density did delay gastric emptying time, but did not affect gluten degradation efficiency. In Chapter 3 of this thesis we critically evaluated the use of paracetamol as postprandial marker for gastric empyting. We showed that paracetamol, when added to a meal and mixed, was divided into two fractions; one fraction encapsulated by the meal and one fraction dissolved in the liquid content of the meal. The rate of absorption of these fractions was different, and both did not accurately reflect gastric emptying rate.

Modulation of the gut microbiota by prebiotics appears to be an interesting approach to enhance gut barrier function, and to treat or even prevent the onset or deterioration of chronic diseases. A limitation of most prebiotics is their rapid fermentation in the proximal colon. Arabinoxylans $(A X)$, the most abundant non-digestible carbohydrates present in wheat, represent an interesting novel class of potential prebiotics. Due to their structure, 
they rely on a whole spectrum of (microbial) enzymes for degradation, resulting in a more distal fermentation and activity. Furthermore, AX structure affects the fermentation pattern and immune modulation, with highest acitivity observed for long-chain AX. In Chapter 4 we aimed to investigate the 6 weeks effect of two different doses high-molecular weight $A X$ on the gut barrier, gut microbiota structure and activity, immune system and metabolic markers in overweight and obese individuals. Compared to placebo, AX intake did not significantly change the relative abundance of the dominant phyla, but a decrease in microbial richness and diversity was observed but only after $15 \mathrm{~g} \mathrm{AX}$ intake, not with the lower dose of $7.5 \mathrm{~g}$ AX. Furthermore, the fecal concentrations of the individual short-chain fatty acids (SCFA) acetate, butyrate and propionate, and of the total SCFA were increased upon intake of AX, compared to the placebo group. Six weeks $7.5 \mathrm{~g}$ AX also significantly lowered fecal $\mathrm{pH}$ compared to placebo. This modulation in gut microbial activity may affect the gut barrier and the gut immune system. An upregulation in the transcription of the junction (TJ) proteins occludin, claudin-3 and claudin-4 in sigmoid biopsies, either after $7.5 \mathrm{~g}$ AX or $15 \mathrm{~g} \mathrm{AX}$ supplementation, was observed. However, no significant changes in TJ protein expression nor in gastrointestinal permeability were found in the AX groups, compared to placebo. In addition, we observed a significant decrease in the production of the pro-inflammatory cytokine TNF $\alpha$ by stimulated peripheral blood mononuclear cells after 6 weeks $15 \mathrm{~g}$ AX intake.

Large scale studies have shown that intake of flavonoid-rich food products has positive effects on cardiovascular diseases (CVD) and their related risk factors. To exert health effects in vivo it is essential that flavonoids are bioavailable, absorbed from the gastrointestinal tract and reach the systemic circulation. Hesperidin, a flavanone glycoside from sweet orange peels, is able to exert positive effects on metabolic and cardiovascular health, but has poor bioavailability in humans. An option to improve the bioavailability of a compound is to improve the dissolution process by micronization. Furthermore, hesperidin has a specific enantiomer configuration; it consists of a mixture of S- and R-enantiomers in a $S: R$ ratio between $1: 1$ and $5: 1$ depending on its source. Stronger biological effects are elicited by the S-enantiomer compared to the R-enantiomer. While in nature the 2Senantiomer is dominant, commercially available hesperidin often contains a mixture of both stereo-isomers. In Chapter 5 we compared the bioavailability of standard hesperidin with that of micronized hesperidin $2 S$ (S:R ratio of 4:1) in a cross-over study in healthy subjects. After ingestion of one dose, micronized hesperidin $2 S$ proved to be more bioavailable than standard hesperidin. This was shown by a significant increase in the cumulative excretion of total hesperitin and of the individual metabolites in 24 hour urine, and by a significant increase in the $\mathrm{AUC}_{0-4 \mathrm{~h}}$ of plasma hesperetin after intake of micronized hesperidin 2S, compared to standard hesperidin. The improved bioavailability of micronized hesperidin $2 S$ may positively affect its biological activity. 
Stimulated by these favourable results, in Chapter 5, in Chapter 6 we studied the effects of 6 weeks micronized hesperidin $2 S$ supplementation on endothelial function, as measured by flow-mediated dilation (FMD), in overweight and obese subjects. Furthermore, also the effects on plasma adhesion molecules, blood pressure and metabolic markers were examined. Besides studying the effects on basal (fasting) endothelial function, we studied the effects of hesperidin $2 \mathrm{~S}$ after inducing temporary, reversible endothelial dysfunction by providing participants a high-fat meal. In this randomized, placebo-controlled, double-blind, parallel-group study we observed no significant changes in fasted nor in postprandial FMD. However, trends towards downregulation of the adhesion molecules sVCAM-1, sICAM- 1 and sP-selectin and towards reduction of both the systolic and diastolic blood pressure were observed after 6 weeks hesperidin $2 S$ intake, compared to placebo. Interestingly, in an exploratory subgroup analysis, excluding those subjects with a highly impaired basal flow-mediated dilation (exclusion of subjects with basal FMD $<3 \%$ ), hesperidin $2 S$ protected from postprandial endothelial dysfunction and significantly downregulated sVCAM- 1 and sICAM-1, as compared to placebo.

In another randomized, placebo-controlled, double-blind, parallel-group study we aimed to assess the 12-week effects of the same hesperidin 25 formulation on gastrointestinal and on systemic metabolic parameters in subjects with features of metabolic syndrome (Chapter 7). In this study, no significant hesperidin-induced effects were observed regarding glucose regulation, blood pressure and blood lipids. However, although fecal total SCFA concentrations did not change, hesperidin $2 S$ influenced the gastrointestinal environment as reflected by a significant shift in fermentation (SCFA) profile from acetate to butyrate, and a marked but non-significant reduction in fecal calprotectin concentrations.

It has also been shown that high intake of carotenoids, lipophilic antioxidants abundant in fruits and vegetables, protects against the development of CVD and CVD related risk factors. The majority of commercially available natural carotenoids are derived from plantbased materials. However, the quality and consistency of these carotenoids varies considerably. Carotenoid-like molecules can also be produced by bacterial species. Those produced by spore-forming bacillus strains are of specific interest. Previous in vitro and animal studies have shown that a specific bacillus strain, named PDO 1, is able to produce gastric-stable carotenoids with superior bioavailability and high antioxidant activity as compared to plant carotenoids. In addition, PDO1 itself may also possess probiotic properties. In Chapter 8 we investigated whether PDO1 is also able to survive transit through the gastroinestinal tract and to release systemically absorbable carotenoids in an in vivo setting. In addition, the gastrointestinal and systemic effects of PDOI and its bacterial carotenoids were studied in a overweight-obese study population. PDO1 was shown to survive transit through the gastrointestinal tract and was able to germinate into vegetative cells under physiological intestinal conditions and to release bioavailable carotenoids in 
vivo. No significant gastrointestinal nor systemic effects were observed after 6 weeks PDO 1 intake, compared to placebo.

In Chapter 9, we presented an overview of the main findings of this thesis, discuss new insights and future perspectives, and provide potential implications for further research. 


\section{Nederlandse samenvatting}

De primaire rol van voeding is kwantitatief en kwalitatief voldoende voedingsstoffen verschaffen zodat er aan de voedingsbehoeften van een individu kan worden voldaan. Daarnaast kan voeding ook gezondheidsbevorderende effecten hebben. Aanvankelijk richtte de voedingswetenschap zich op het identificeren van essentiële elementen vit de voeding en hun rol in de preventie en behandeling van diverse deficiëntieziekten. In de afgelopen jaren ligt de wetenschappelijke en klinische focus meer op de rol van voeding in ziekten geassocieerd met overmatige voedselinname. De ontdekking en ontwikkeling van functionele voedingsmiddelen heeft "functional foods" tot een snelgroeiend onderdeel van de voedselindustrie gemaakt. Functionele voedingsmiddelen worden gedefinieerd als voedingsmiddelen of voedselingrediënten die één of meer lichamelijk functies doelgericht beïnvloeden met een gezondheidsbevorderend effect, of wanneer het product een fysiologisch effect heeft of effect op psychisch functioneren anders dan het traditionele nutritionele effect. De rol van functionele voeding in ziektepreventie en gezondheidsbevordering is tegenwoordig een belangriik onderwerp van onderzoek.

In dit proefschrift onderzochten we de effecten van verschillende voedingsinterventies op de gastro-intestinale en metabole gezondheid van gezonde individuen en van individuen met overgewicht en obesitas. We bestudeerden de effecten van een prolyl endoprotease, een potentieel prebiotisch en probiotisch product, en van twee verschillende fytonutriënten. Gluten, een eiwit riik aan proline, wordt slecht afgebroken in het maag-darmstelsel door het ontbreken van proline splitsende proteasen in het maag-darmkanaal. De aanwezigheid van lange prolinerijke glutenpeptiden in de dunne darm kan meerdere gluten-gerelateerde aandoeningen via verschillende mechanismen induceren. Prolyl endoproteasen zijn enzymen die het vermogen hebben om de prolineriike glutenpeptiden te splitsen. In Hoofdstuk 2 onderzochten we de in vivo werkzaamheid van Aspergillus Niger prolyl endoprotease (AN-PEP) in het afbreken van gluten. AN-PEP was in staat om gluten, verwerkt in een maaltijd, efficiënt af te breken in de maag van gezonde vrijwilligers voordat deze gluten de dunne darm bereikten. Daarnaast zorgde het verhogen van de calorische dichtheid van de maaltiid tot een vertraging in maagontledigingssnelheid, maar had het geen invloed op de efficiëntie van glutenafbraak. In Hoofdstuk 3 van dit proefschrift evalueerden we het gebruik van paracetamol als postprandiale marker voor maagontleding. We toonden aan dat paracetamol, wanneer toegevoegd en vermengd met een maaltijd, wordt onderverdeeld in twee fracties; één paracetamolfractie ingekapseld door het vaste deel van de maaltiid en één fractie opgelost in de vloeibare inhoud van de maaltijd. De snelheid van absorptie van deze fracties is verschillend, en geen van beiden geeft nauwkeurig de maagledigingssnelheid weer. 
Het veranderen van de samenstelling van darmmicrobiota door prebiotische producten lijkt een interessante manier om de darmbarrièrefunctie te verbeteren en om het ontstaan of de progressie van chronische ziekten te behandelen of zelfs te voorkomen. Een beperking van de meeste prebiotica is hun snelle fermentatie in het proximale colon. Arabinoxylanen (AX), de meest voorkomende niet-verteerbare koolhydraten in tarwe, vormen een interessante nieuwe klasse van potentiële prebiotica. Door hun structuur hebben ze een breed spectrum van (bacteriële) enzymen nodig voor hun afbraak, hetgeen resulteert in een meer distale fermentatie en activiteit. Bovendien beïnvloedt hun structuur het fermentatiepatroon en bepaalt het de mate van immuunmodulatie, met de hoogste activiteit waargenomen voor lange-keten AX. In Hoofdstuk 4 onderzochten we de effecten van twee verschillende doseringen $A X$, na 6 weken toediening, in gezonde personen met overgewicht of obesitas. Uitkomstmaten waren darmbarrièrefunctie, samenstelling en activiteit van de darmmicrobiota, het immuunsysteem en metabole parameters. In vergelijking met placebo bleek AX de relatieve aanwezigheid van de dominante bacteriefyla in feces niet significant te beïnvloeden. Wel werd er een afname in microbiële rijkdom en diversiteit waargenomen na 6 weken $15 \mathrm{~g} A X$ inname, maar niet na 6 weken 7,5 g AX inname. Daarnaast waren de fecale concentraties van de verschillende korteketenvetzuren acetaat, butyraat en propionaat en van de totale hoeveelheid korteketenvetzuren verhoogd na inname van 6 weken $A X$, tegenover een daling in de placebogroep. Zes weken inname van 7,5 g AX zorgde voor een significante verlaging van de fecale $\mathrm{pH}$, in vergelijking met placebo. Deze modulatie van microbiële activiteit in de darm zou de darmbarrièrefunctie en het immuunsysteem kunnen beïnvloeden. Ook werd er een toename van transcriptie van de tight junction (TJ) eiwitten occludine, claudine-3 en claudine-4 in sigmoid biopten waargenomen, zowel na 7,5 g AX als na $15 \mathrm{~g}$ AX suppletie. Echter, er werden geen significante veranderingen in TJ eiwitexpressie of in gastro-intestinale permeabiliteit gezien na AX suppletie, in vergelijking met placebo. Tenslotte zagen we in deze studie een significante daling in de productie van het pro-inflammatoire cytokine TNF-alfa door gestimuleerde perifere mononucleaire bloedcellen na 6 weken inname van $15 \mathrm{~g}$ AX vergeleken met inname van placebo.

Studies van grote omvang hebben aangetoond dat flavonoïde-rijke producten gunstige effecten hebben op hart- en vaatziekten en de bijbehorende risicofactoren. $\mathrm{Om}$ in vivo gezondheidseffecten te bewerkstelligen is het essentieel dat flavonoïden goed biobeschikbaar zijn, met andere woorden dat ze worden opgenomen vanuit het maagdarmstelsel en dat ze de systemische circulatie bereiken. Hesperidine, een flavonoïde glycoside uit sinaasappelschillen, kan wel degelijk positieve effecten vitoefenen op metabole en cardiovasculaire gezondheid, maar is in mensen maar in geringe mate biologisch beschikbaar. Een manier om de biologische beschikbaarheid te verbeteren is door het proces van oplossen te verbeteren middels micronisatie. Daarnaast heeft hesperidine een specifieke enantiomeer configuratie; het bestaat uit een mengsel van S- en 
R-enantiomeren in een S:R-verhouding tussen 1:1 en 5:1, afhankelijk van de bron. De Senantiomeer heeft een sterker biologisch effect dan de R-enantiomeer. Terwill in de natuur de 2S-enantiomeer overheerst, bevatten commercieel verkrijgbare hesperidine producten vaak een mengsel van beide stereo-isomeren. In Hoofdstuk 5 vergeleken we de biologische beschikbaarheid van standaard hesperidine met die van gemicroniseerde hesperidine $2 \mathrm{~S}$ (S:R-verhouding van 4:1) in een cross-over studie met gezonde proefpersonen. Na inname van één dosis bleek de gemicroniseerde hesperidine $2 \mathrm{~S}$ beter biologisch beschikbaar te zijn dan de standaard hesperidine. Dit werd aangetoond middels een significante toename van de cumulatieve uitscheiding van totaal hesperetine en van de individuele hesperidinemetabolieten in 24-uurs urine. Daarnaast was er een significante toename van de $A \cup C_{0-4 h}$ van plasma hesperetine na inname van de gemicroniseerde hesperidine $2 S$, in vergelijking met standaard hesperidine. De verbeterde biologische beschikbaarheid van gemicroniseerd hesperidine $2 S$ zou tevens de biologische activiteit gunstig kunnen beïnvloeden.

Naar aanleiding van bovengenoemde gunstige resultaten, bestudeerden wij in Hoofdstuk 6 de effecten van 6 weken gemicroniseerde hesperidine $2 S$ inname op endotheelfunctie, gemeten met flow-gemedieerde dilatatie, in personen met overgewicht en obesitas. Daarnaast onderzochten we ook de effecten op plasma adhesiemoleculen, bloeddruk en metabole parameters. Naast het bestuderen van de effecten op basale (nuchtere) endotheelfunctie, bestudeerden wii ook de effecten van hesperidine $2 S$ na inductie van tijdelijke, reversibele endotheeldysfunctie door deelnemers een vetriike maaltiid te geven. In deze gerandomiseerde, placebo-gecontroleerde, dubbelblinde, parallelle studie zagen we geen significante veranderingen in nuchtere noch in postprandiale flow-gemedieerde dilatatie. Wel zagen we een dalende trend in de adhesiemoleculen sVCAM- 1, sICAM- 1 en sP-selectine en in de systolische en diastolische bloeddruk na 6 weken hesperidine $2 \mathrm{~S}$ inname, vergeleken met placebo. Daarnaast zagen we in een subgroep analyse dat hesperidine $2 S$ een beschermend effect had ten aanzien van postprandiale endotheeldysfunctie, en dat het de adhesiemoleculen sVCAM- 1 en sICAM-1 significant verlaagde, in vergelijking met de placebo. In deze subgroep analyse waren personen met een sterk verminderde basale flow-gemedieerde dilatatie $(<3 \%)$ geëxcludeerd omdat verbetering van endotheelfunctie na een dergelijke korte termijn voedingsinterventie niet te verwachten is in personen met zo'n chronisch slecht functionerend endotheel. In een volgend gerandomiseerd, placebo-gecontroleerde, dubbelblinde, parallelle studie onderzochten we de effecten van hetzelfde hesperidine $2 S$ supplement op gastrointestinale en systemische metabole parameters bii patiënten met kenmerken van het metabool syndroom na 12 weken inname (Hoofdstuk 7). In deze studie werden geen significante hesperidine-geïnduceerde effecten waargenomen ten aanzien van de glucoseregulatie, bloeddruk en lipidenconcentraties. Hoewel de totale korteketenvetzurenconcentratie in feces niet veranderde, beïnvloedde hesperidine $2 S$ het gastro- 
intestinale milieu, weerspiegeld door een significante verschuiving in het fermentatie (korteketenvetzuren) profiel van acetaat naar butyraat. Daarnaast werd er een duidelijke maar niet-significante daling gezien in fecale calprotectine concentraties.

Carotenoïden zijn lipofiele antioxidanten overvloedig aanwezig in fruit en groenten. Het is aangetoond dat een hoge inname van carotenoïden beschermt tegen de ontwikkeling van hart- en vaatziekten en gerelateerde risicofactoren. De meeste commercieel verkrijgbare natuurlijke carotenoïden zijn afkomstig van plantaardige materialen. Echter, de kwaliteit en consistentie van deze carotenoïden varieert aanzienlijk. Carotenoïden kunnen ook worden geproduceerd door bacteriën, met name carotenoïden die geproduceerd worden door sporenvormende bacillus-stammen zijn van belang. Eerdere in vitro en dierstudies hebben aangetoond dat een specifieke bacillus-stam, genaamd PDO 1, in staat is maagsapresistente carotenoïden met superieure biobeschikbaarheid en hoge antioxidant werking te produceren in vergelijking met carotenoïden afkomstig van planten. Daarnaast bezit PDO 1 zelf mogeliik ook probiotische eigenschappen. In Hoofdstuk 8 onderzochten wij of PDO 1 in staat is om in vivo te overleven in het maagdarmstelsel en in staat is tot productie van systemisch opneembare carotenoïden. Daarnaast bestudeerden wij de gastro-intestinale en systemische effecten van PDOl en van de geproduceerde bacteriële carotenoïden in individuen met overgewicht en obesitas. PDO1 bleek in vivo in staat te overleven in het menselijke maagdarmstelsel en te ontkiemen tot vegetatieve cellen met afgifte van biobeschikbare carotenoïden. Echter, er werden geen significante gastro-intestinale noch systemische effecten waargenomen na 6 weken PDO 1 inname, in vergeliiking met placebo.

In Hoofdstuk 9 gaven we een overzicht van de belangrijkste bevindingen van dit proefschrift, bediscussieerden we nieuwe inzichten en toekomstperspectieven, en droegen we mogelijke implicaties voor verder onderzoek aan. 


\section{Valorisation}

Valorisation is the process of value-creation out of knowledge, by making this knowledge suitable and available for economic or societal utilization and to translate it into highpotential products, services, processes and industrial activity.' With this thesis, we aim to improve our understanding of the effects of several nutritional interventions on gastrointestinal and metabolic health, and to make the acquired knowledge and expertise available for society.

The relationship between food and health is complex, being multifactorial and multidimensional. Food provides sufficient quantitative and qualitative supply of nutrients and supplies the human body with materials that can be used for the construction and repair of structures, tissues and organs. Furthermore, the role of functional foods in disease prevention and health promotion is emerging. Functional foods are defined as foods or food ingredients that affect one or more functions in the body in a targeted way so that positive effects on health are to be expected, or when the component has a physiological or psychological effect beyond the traditional nutritional effect. ${ }^{2,3}$ On the other hand, food can also negatively influence health and initiate or contribute to illness. To date, in many low- and middle income countries access to food is still limited and the availability and quality of nutrition is poor, leading to severe undernourishment and food insecurity. ${ }^{4}$ In contrast, the prevalence of overweight and obesity, linked to excess food intake and 'overnutrition', has worldwide more than doubled since 1980. In 2014, approximately $39 \%$ of people aged 18 years and over were overweight and $13 \%$ obese. 5 Also in the Netherlands, the number of individuals with overweight and obesity is tremendous; in 2015,50.3\% of the adults aged 20 years and older were overweight and $13.7 \%$ were obese. ${ }^{6}$ Overweight and obesity are associated with a broad spectrum of gastrointestinal and systemic metabolic alterations, serving as major risk factors for the development of chronic metabolic diseases such as diabetes mellitus type 2 and cardiovascular disease. ${ }^{7.8}$ As a consequence, obesity imposes a tremendous economic burden on society through increased total direct (treatment obesityassociated diseases) and indirect (productivity losses) medical costs. In the Netherlands, it has been calculated that in general the direct health care costs of obesity account for up to total $2.2 \%$ of national health expenditure. ${ }^{9}$ The indirect health care costs could amount to twice the direct health care costs. ${ }^{10}$ Significant reductions in these costs can be accomplished by prevention of overweight and obesity or by a significant delay in the onset of overweight, obesity and associated diseases.

Besides diseases linked to undernourishment and overnutrition, there exists another class of diseases related to food: food allergies and food intolerances. Important and epidemiologically relevant phenomenon are the gluten related disorders, such as wheat allergy, celiac disease and gluten sensitivity. The number of individuals embracing a gluten- 
free diet is increasing. However, a gluten-free diet is hard to comply with as gluten-free products may not always be correctly labelled, are expensive, and may not always be at hand during social events or travelling. Accordingly, the market for gluten-free products is constantly growing and research identifying alternative therapies is ongoing.

The aim of this thesis was to investigate the effects of several nutritional interventions on gastrointestinal and metabolic health in healthy, overweight and obese individuals. The nutritional interventions we studied included a prolyl endoprotease, a putative prebiotic supplement, a putative probiotic species, and two different phytonutrients.

Gluten is the main structural protein complex of wheat with equivalent toxic proteins present in barley and rye. These gluten-containing cereals have always been a main component of the Western diet, but their consumption is increasing in Eastern countries as well. Ingestion of gluten can induce a wide range of gluten related disorders with different pathogenic pathways. For all conditions, strict adherence to a gluten-free diet is still the only effective treatment. As such, the number of individuals on a gluten-free diet is increasing worldwide. However, the high cost and often poor availability and palatability of gluten-free products makes it difficult to maintain such diet on the longer term. Even in case of strict adherence, a subgroup of individuals may remain symptomatic due to inadvertent gluten indigestion. In addition, a gluten-free diet is nutritionally suboptimal, it may predispose to an undesirable high intake of fat and carbohydrates and lead to poor vitamin status and detrimental weight gain or weight loss. Due to these problems with gluten-free dieting a strong interest in the development of alternative therapies exists. A possible alternative treatment is enzyme supplementation therapy targeted at degradation of immunogenic gluten peptides before entering the intestinal epithelium. In this thesis, we evaluated the in vivo glutendegrading potential of Aspergilles Niger-derived prolyl endoprotease (AN-PEP). To standardize each meal intake, we intragastrically administered the test meal via a triple lumen nasogastroduodenal tube. AN-PEP showed to be able to efficiently degrade gluten from a meal in the stomach of healthy volunteers within the time period a meal normally resides in the stomach. The gluten-degrading efficacy of AN-PEP was independent of meal caloric density and gastric emptying rate. These in vivo results are promising but to optimise AN-PEP for clinical application it is important that future studies investigate the efficacy of AN-PEP in an actual meal setting, focusing on subjects intolerant to gluten. Furthermore, it is anticipated that these supplements will not fully replace the gluten-free diet, but will be marketed as a supplement that in conjunction with a gluten-free diet may help digest unintentional ingested gluten for subjects intolerant to gluten.

To measure gastric emptying rate in the above-mentioned study, we used paracetamol as post-prandial marker molecule. This method is considered a well-established method and is extensively applied in research. It is assumed that paracetamol, mixed trough a meal and 
ingested, will be absorbed immediately in the duodenum after leaving the stomach and that its passage time is identical to that of the meal. The gastric emptying rate can be derived from the time course of the plasma paracetamol concentration. However, in our study we obtained data which were in conflict with this widely accepted model. We observed that paracetamol was divided in two fractions; one fraction dissolved in the liquid content of the meal and another fraction encapsulated by meal contents that precipitate in the acid stomach environment. The aqueous phase is expected to leave the stomach quickly and the paracetamol is readily absorbed in the duodenum, the encapsulated paracetamol is slowly released from the bolus and not instantaneously taken up once it reaches the duodenum. Both fractions did not accurately reflect gastric emptying rate, instead these data show the effect of food on the rate of absorption of paracetamol. The absorption of a drug in the gastrointestinal tract bears major clinical relevance. Food-drug interactions are thus not limited to an effect of food on gastric emptying, but it also includes bioaccessibility. Bioaccessibility, i.e. the release of a compound from the matrix in the intestine, has an impact on both the onset and duration of the pharmacotherapeutic effect of a drug.

The human gut is host to a diverse and dynamic community of several trillion microbes that play an important role in intestinal physiology, nutrient digestion, luminal metabolism and immune function." Colonization of the gastrointestinal tract is influenced by genetic background, gestational age, mode of delivery, type of feeding, exposure to antimicrobials and, very important, diet throughout life. ${ }^{12}$ Long-term perturbations to the intestinal environment can induce changes in the gut microbiome, contributing to the development of a variety of gastrointestinal diseases ${ }^{13}$ as well as systemic metabolic diseases including diabetes mellitus type 2 and cardiovascular disease. ${ }^{14}$ Thus, targeting the gut microbiome seems to offer a promising strategy to ameliorate or even prevent the development of above-mentioned diseases. A number of dietary strategies potentially modulating the gut microbiome are available; pre- and probiotics are among the most well established, while recent data suggest that also different types of polyphenolic compounds may influence the gut microbiome.

In this thesis we evaluated the effect of a high-molecular weight arabinoxylan (AX) concentrate, a potential prebiotic product, on intestinal microbiota, gut barrier and immune function in overweight and obese subjects. AX form an interesting novel class of potential prebiotics as, due to their structure, they are more gradually fermented along the colon resulting in a more distal fermentation and activity. A limitation of most other prebiotics is their rapid fermentation (and thus activity only) in the proximal colon. Previous in vitro and animal studies have shown promising effects of this product on the gut barrier, gut microbiota, immune system and metabolic markers. In humans, AX administration did not result in a change in the overall microbiota composition, but $A X$ resulted in significantly increased fecal concentrations of short-chain fatty acids (SCFA) and lower fecal pH. The 
effects of $A X$ on the gut barrier were explored at various levels. At gene transcription level, we found a significant upregulation of the tight junction (TJ) proteins occludin, claudin-3 and claudin-4, important transmembrane components regulating paracellular permeability. However, these changes in TJ gene expression were nog accompagnied with changes in TJ protein expression or in gastrointestinal permeability. Furthermore, results of this study suggest a capacity of $A X$ to influence the immune function. At last, daily intake of $A X$ for several weeks proved to be well-tolerated by the participants. Altogether, these data suggest that also in humans high-molecular weight $A X$ modulates the gut microbiome and thereby may positively affect gut barrier and immune function. While more research in large, homogeneous study populations is required to confirm its in vivo microbiota modulating and potentially gut barrier and immune system modulating- properties, $A X$ is considered a promising prebiotic compound conferring health effects along the entire length of the colon. In this thesis, we also studied the effects of a putative probiotic product, a Bacillus indicus strain called PDO1, on the intestinal microbiota and gut barrier in healthy, yet overweight and obese individuals. Several studies have already shown potential health benefits of bacillus strains. In addition, bacillus species as probiotics are particularly attractive for functional reasons as they can form robust endospores that are able to survive transit through the stomach, are stable to many food processing steps and can be long-term stored at room temperature. Previous in vitro data showed that this strain was able to survive and germinate in the gastrointestinal tract and to modulate the intestinal environment. In vivo, PDO1 also survived transit throughout the digestive tract, but did not significantly influence the gut microbiome or gut barrier when compared to placebo. Repeated intake of PDO 1 was well-tolerated by the participants, and was even accompanied with a decrease in reported gastrointestinal symptoms such as borborygmi, abdominal distension, eructation and increased flatulance. Previous studies in IBS patients showed that changing the gut microbiome by probiotics may induce quantitative and qualitative decreases in bacterial groups with gas-producing abilities. ${ }^{15,16}$ Possibly, a similar mechanism played a role in our study results. Although no significant effects of PDO1 on gut microbial environment or gut barrier function could be established in this study, it does provide the first evidence in humans that PDO 1 survives transit through the gastrointestinal tract and is well-tolerated. To determine whether PDO1 possesses additional probiotic properties, it is important that more in vivo studies will be conducted in future, including a homogeneous, more diseased study population or that a stressor is applied during the test period inducing an imbalance in homeostasis.

As said before, also polyphenols may have the potential to affect the intestinal environment. We evaluated the relationship between the polyphenol hesperidin and SCFA production. Although we did not observe an effect on the quantity of SCFA production, twelve weeks hesperidin intake modulated the intestinal environment as reflected by a shift from the SCFA acetate to the health-promoting SCFA butyrate. These results strengthen the 
hypothesis that also polyphenols are able to influence the gut microbiome. Research investigating the interactions between the gut microbiome and phenolic compounds is still in its early days. More human intervention studies are required to understand the underlying mechanisms and to establish the potential prebiotic-like effects of hesperidin and other polyphenols.

It has been demonstrated that fruits, vegetables and grains exert protective effects against the development of chronic metabolic diseases such as diabetes mellitus type 2 and cardiovascular disease. This protective role can be mainly attributed to the antioxidant phytonutrients in them. Today, about 10,000 phytonutrients have been identified. In this thesis we studied the bioavailability and systemic effects of two main kinds of phytonutrients: polyphenols and carotenoids.

Hesperidin, a polyphenol abundantly present in the peels of citrus fruits, has drawn attention due to its positive effects on metabolic and cardiovascular health. In order to exert systemic health effects, it is essential that a compound is bioavailable and absorbed from the gastrointestinal tract into the systemic circulation. However, the bioavailability of hesperidin in humans is low. We demonstrated that the bioavailability of a micronized hesperidin $2 S$ formulation, having a similar enantiomer configuration as natural hesperidin, is higher than that of a non-micronized standard hesperidin product. The enhanced bioavailability may positively affect its biological activity. Furthermore, this study provided evidence that there exists a high inter-individual variation in polyphenol bioavailability, most likely the result of a great inter-individual diversity in the gut microbiome.

Based on above results, we investigated the effect of micronized hesperidin $2 S$ supplementation on cardiovascular and metabolic parameters in healthy, yet overweight and obese individuals. Results indicate a promising preventive role for hesperidin in cardiovascular disease. Given the high inter-individual variability of polyphenol bioavailability in humans, it would be particularly important and interesting to correlate the magnitude of observed changes with plasma polyphenol concentrations in future studies. Regarding glucose and lipid metabolism we did not observe hesperidin-induced beneficial effects in our generally healthy study population. Consequently, long-term intervention studies in subjects with(pre)diabetes, metabolic syndrome or hypercholesterolemia are warranted to establish and confirm beneficial hesperidin-induced effects on systemic metabolic health markers.

Carotenoids are lipophilic antioxidants, synthesized mainly in plants, but also by a number of bacterial species. In contrast to plant-derived carotenoids, bacterial carotenoids are gastricstable, are equally or even more bioaccessible and bioavailable, and have a higher antioxidant capacity. In this thesis we evaluated the bioavailability of carotenoids, produced by a spore-forming Bacillus strain, in overweight and obese individuals. The bacterial carotenoids were present in the plasma and levels increased throughout the study period. 
These results indicate that the bacillus strain was able to produce systemically absorbable bacterial carotenoids in vivo. However, no beneficial effects of the bacterial carotenoids regarding cardiovascular and metabolic parameters were observed, probably due to the fact that participants had normal baseline values for most parameters. It is clear that more and larger human intervention studies are needed to investigate and confirm an improved bioavailability, antioxidant capacity and systemic health effects of bacterial carotenoids over plant-derived carotenoids.

In conclusion, beyond its nutritional value food can also play an important role in disease prevention and health promotion. All of the here studied nutritional interventions may affect one or more functions in the body in a positive way and therefore can be considered a functional food. Health-conscious consumers are increasingly seeking functional foods in an effort to control their own health and well-being. However, the field of functional foods is still in its infancy. Many functional foods showed to have beneficial effects in in vitro and in animal studies, but their efficacy in humans remains equivocal. A number of factors complicate the establishment of a strong scientific foundation for a functional food. Factors include complexity of the food substance, compensatory metabolic changes that may occur with dietary changes, lack of surrogate markers of disease development and, very important, the existing large variation between individuals regarding general health, genetic make-up and expression, gut microbiome and environmental factors. The development of nutritional therapies that target on prevention or on improvement of chronic gastrointestinal, cardiovascular and metabolic diseases is important and is considered an attractive alternative for medication use. Therefore, it is essential that in future more large, welldesigned, placebo-controlled randomized trials exploring the effects of nutritional interventions in well-defined study populations will be performed. Besides scientifically proven health effects, also consumer acceptance is key success factor for use of functional foods in the future. As a result, within the food industry, consumer behavior is currently an important research topic. 


\section{References}

1. Nederland Ondernemend Innovatieland 2009

2. Bellisle F, Blundell JE, Dye L, et al. Functional food science and behaviour and psychological functions. Br J Nutr. 1998;80 Suppl 1:S173-93.

3. Clydesdale FM. A proposal for the establishment of scientific criteria for health claims for functional foods. Nutr Rev. 1997; 55(12):413-22.

4. Nations, F.a.A.O.o.t.U. The state of food insecurity in the world 2015. 2015; Available from: http://www.fao.org/hunger/en/.

5. Van Gaal LF, Mertens IL, De Block CE. Mechanisms linking obesity with cardiovascular disease. Nature. 2006; 444(7121):875-80

6. Wilson PW, D'Agostino RB, Parise H, et al. Metabolic syndrome as a precursor of cardiovascular disease and type 2 diabetes mellitus. Circulation. 2005; 1 12(20):3066-72.

7. Organization WH. Obesity and overweight. Fact sheet No. 211. http://www.who.int/mediacentre/ factsheets/fs3 1 1/en/: World Health Organization Media Centre; 2015.

8. Gezondheidsmonitor GGD'en CeR. Gezondheidsmonitor Volwassenen. In: RIVM, editor. 2015.

9. Zorgkosten van ongezond gedrag. RIVM rapport.

10. Organization WH. Obesity: preventing and managing the global epidemic. Report of a WHO Consultation. WHO Technical Report Series, No. 894: World Health Organization; 2000. Available from: http://www.who.int/nutrition/publications/obesity/en/index.html.

11. Guinane CM, Cotter PD. Role of the gut microbiota in health and chronic gastrointestinal disease: understanding a hidden metabolic organ. Therap Adv Gastroenterol. 2013;6(4):295-308.

12. Chan YK, Estaki M, Gibson DL. Clinical consequences of diet-induced dysbiosis. Ann Nutr Metab. 2013; 63 Suppl 2:28-40.

13. Nagao-Kitamoto H, Kitamoto S, Kuffa P, Kamada N. Pathogenic role of the gut microbiota in gastrointestinal diseases. Intest Res. 2016; 14(2): 127-38.

14. Moreno-Indias I, Cardona F, Tinahones FJ, et al. Impact of the gut microbiota on the development of obesity and type 2 diabetes mellitus. Front Microbiol. 2014; 5: 190.

15. Johansson ML, Molin G, Jeppsson B, et al. Administration of different Lactobacillus strains in fermented oatmeal soup: in vivo colonization of human intestinal mucosa and effect on the indigenous flora. Appl Environ Microbiol. 1993;59(1): 15-20.

16. Quigley EM. From comic relief to real understanding; how intestinal gas causes symptoms. Gut. 2003; 52(12):1659-61. 
232 


\section{List of publications}

\section{Published articles}

Salden BN*, Monserrat V*, Troost FJ, Bruins MJ, Edens L, Bartholomé R, Haenen GR, Winkens B, Koning F, Masclee AA. * Both authors contributed equally. Randomised clinical study: Aspergillus niger-derived enzyme digests gluten in the stomach of healthy volunteers. Aliment Pharmacol Ther. 2015 Aug;42(3):273-85. doi: 10.1111 1/apt.13266.

Salden BN*, Monserrat V*, Troost FJ, Bruins MJ, Edens L, Bartholomé R, Haenen GR, Winkens B, Koning F, Masclee AA. * Both authors contributed equally. Editorial: enhancing gluten digestion in the stomach - a further help to minimise unintentional ingestion? Authors reply. Aliment Pharmacol Ther. 2015 Aug;42(4):485. doi: 10.1 11 1/apt. 13296.

Salden BN*, Monserrat V*, Troost FJ, Bruins MJ, Edens L, Bartholomé R, Haenen GR, Winkens B, Koning F, Masclee AA. * Both authors contributed equally. Letter: gluten digestion in the stomach and duodenum by Aspergillus niger-derived enzyme - things to ponder. Authors' reply. Aliment Pharmacol Ther. 2015 Oct;42(7):946-7. doi: 10.1111 /apt. 13382.

Bartholomé R, Salden B, Vrolijk MF, Troost FJ, Masclee A, Bast A, Haenen GR. Paracetamol as a post prandial marker for gastric emptying, a food-drug interaction on absorption. PLoS One. 2015 Sep 9; 10(9):e0136618. doi: 10.1371/journal.pone.0136618.

Le Clerca CM, Mooi RJ, Winkens B, Salden BN, Bakker CM, van Nunen AB, Keulen EP, de Ridder RJ, Masclee AA, Sanduleanu S. Temporal trends and variability of colonoscopy performance in a gastroenterology practice. Endoscopy. 2016 Mar;48(3):248-55. doi: 10.1055/s-0041- 111117 .

Salden BN, Troost FJ, de Groot E, Stevens YR, Garcés-Rimón M, Possemiers S, Winkens B, Masclee AA. Randomized clinical trial on the efficacy of hesperidin $2 S$ on validated cardiovascular biomarkers in healthy overweight individuals. Am J Clin Nutr. 2016 Oct 26. pii: ajen 136960 .

Salden BN, Troost FJ, Wilms E, Truchado P, Vilchez-Vargas R, Pieper DH, Jáuregui R, Marzorati M, van de Wiele T, Possemiers S, Masclee AA. Reinforcement of intestinal epithelial barrier by arabinoxylans in overweight and obese subjects: a randomized controlled trial. Clinical Nutrition, article in press. 


\section{Submitted manuscripts}

Truchado P, Hernandez-Sanabria E, Salden BN, van den Abbeele P, Vilchez-Vargaz R, Jauregui R, Pieper DH, Possemiers S, van de Wiele T. Long chain arabinoxylans shift the mucosa-associated microbiota in the proximal colon of the Simulator of the Human Intestinal Microbial Ecosystem (M-SHIME).

Overdevest E, Wouters J, Mouithys-Mickalad A, Franck T, Wolfs K, Salden BN, Possemiers S. Citrus flavonoid extract improves human exercise performance through improvement of mitochondrial respiration efficiency.

\section{Abstracts (selection)}

Salden BN, Bouwens M, WInkens B, Rondagh E, Riedl R, Kaltenbach T, Soetikno RM, Masclee AA, Sanduleanu S. Sal109 Systematic Training Improved the Quality of Colonoscopy by Reducing the Variation of Quality Indicators Among Providers. Gastroenterology 2012; 142: S-218.

Salden BN, van Osch L, Hoving C, Laven M, Holtkamp K, Gulikers S, Masclee AA, Sanduleanu S. Mo 1093 Differences in Quality of Life Communication Behavior and Quality of Care Between Patients With Familial CRC Versus Lynch Syndrome. Gastroenterology 2013; 144: S-575

Salden BN*, Monserrat V*, Troost FJ, Bruins MJ, Edens L, Bartholomé R, Haenen GR, Winkens B, Koning F, Masclee AA. * Both authors contributed equally. Su2096 Gluten Degrading Enzyme Effectively Digests Gluten in the Stomach and Small Intestine of Healthy Volunteers. Gastroenterology 2014; 146: S-545

Le Clercq CM*, Mooi FJ*, Bouwens MW, Salden BN, Winkens B, de Ridder RJ, Bakker CM, van Nunen A, Keulen E, Masclee AA, Sanduleanu S. * Both authors contributed equally. 170 Variation and Time Trends in Quality of Colonoscopic Examination. Gastrointest Endosc 2014; 9: $A B 118-A B 119$.

Salden BN, Troost FJ, Wilms E, Brüll F, Truchado P, van de Wiele T, Possemiers S, Masclee AA. 1045 Arabinoxylans Show Distinct Prebiotic Properties and May Affect Intestinal Barrier Function. Gastroenterology 2015; 148: S- 197. 


\section{Curriculum vitae}

Bouke Nicolette Hubertus Salden was born on June $17^{\text {th }} 1987$ in Nieuwstadt, The Netherlands. After graduation from the Trevianum Gymnasium in Sittard in 2005, she started medical school at the Maastricht University, Faculty of Health, Medicine and Life Sciences. During medical school she finished clinical and scientific traineeships at the Division of Gastroenterology and Hepatology, Maastricht University Medical Center. After graduating in 2011 , Bouke started as PhD-fellow affiliated to the division of Gastroenterology and Hepatology of Maastricht University Medical Center, and to NUTRIM, the School of

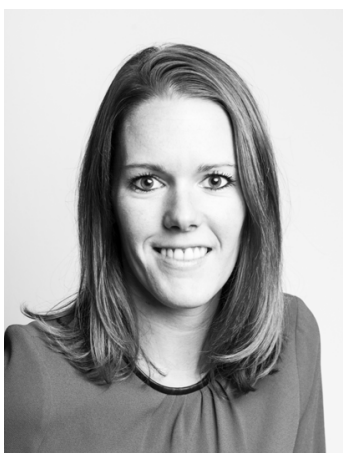
Nutrition and Translational Research in Metabolism, Maastricht University, under the supervision of prof. dr. A.A.M. Masclee, Dr. F.J. Troost and Dr. S. Possemiers. Part of her PhD was conducted in collaboration with BioActor BV, where she acted as Medical Affairs Manager. In September 2016 she started her residency Gastroenterology-Hepatology under supervision of Prof. dr. A.A.M. Masclee. She is currently working at the Internal Medicine department at the Catharina Hospital Eindhoven under supervision of Dr. C.J.A.M. Konings. 


\section{Dankwoord}

Eindeliik kriig ik dan de kans om de mensen te bedanken die - direct of indirect - hebben bijgedragen aan dit proefschrift. Het is een vitdaging om de waarde van jullie bijdrage en mijn dank hiervoor in een paar regels uit te drukken, maar ik ga een poging wagen!

Prof. Masclee, beste Ad, ik ben ontzettend blij dat u mij de mogelijkheid heeft gegeven te promoveren. Ik kan me herinneren dat wij beiden in het begin twijfelden of een promotietraject voor mij wel de juiste keuze was op dat moment. Met het AN-PEP project gaf u mij de kans om een tijdje van het leven als onderzoeker te proeven. Daarna was het duidelijk voor mij: ik wilde dit uitbouwen tot een promotietraject. Ik wil u bedanken voor alle hulp, de goede begeleiding en steun, en voor het snelle nakijken van manuscripten, zeker in de laatste fase van mijn promotie.

Dr. Troost, beste Freddy, bedankt voor al je hulp en gezelligheid in de afgelopen jaren. Met name in de opstartperiode heb ik veel van je geleerd, daarna gaf je me de vrijheid mijn eigen pad te volgen. Onze vaste dinsdagmiddagbespreking werd steeds minder noodzaak, maar bleef altijd een goed excuus om gezellig samen een kop koffie te drinken.

Dr. Possemiers, beste Sam, naast co-promotor was je ook mijn wetenschappelijk supervisor binnen BioActor. Ik heb in de afgelopen jaren zowel op wetenschappelijk als op commercieel vlak veel van je geleerd. Bedankt dat ik altijd voor alles bii je terecht kon, en je meestal snel en constructief commentaar gaf op mijn vragen, METC-protocollen en manuscripten. Daarnaast was je altijd fijn gezelschap op de vele BioActor-tripies die we samen gemaakt hebben.

Graag wil ik de leden van de beoordelingscommissie, prof. dr. K. Venema, dr. D. Jonkers, prof. dr. C.J.J. Mulder, prof. dr. J. Plat en prof. dr. R.F. Witkamp bedanken voor het kritisch beoordelen van mijn proefschrift, het zitting nemen in de promotiecommissie en voor hun bijdrage aan deze biizondere dag.

BioActor BV wil ik bedanken voor de mogeliikheid om als promovendus tegelijkertijd de functie van clinical research manager binnen dit bedriff te bekleden.

Hans, als managing director van BioActor heb ik veel met jou samengewerkt. Niet altijd waren wij het met elkaar eens, maar we kwamen er viteindelijk toch altijd uit. Ik bewonder je om je tomeloze energie, dat zich onder andere uit in altijd nieuwe onderzoeksideeën. Nooit zit je lang bij de pakken neer als de data niet zijn zoals gehoopt. Met mijn functie als clinical research manager maakte iii me verantwoordelijk voor alle onderzoeksprojecten binnen het bedriif. Ik leerde zelfstandig werken, delegeren, grenzen stellen en bewaken, ik 
mocht naar meetings van Europese projecten en moest vele presentaties geven. Ik leerde wat het inhoudt om voor een bedriff te werken en het heeft ervoor gezorgd dat ik de afgelopen jaren zelfverzekerder, zelfstandiger en assertiever ben geworden. Bedankt daarvoor!

Uiteraard ben ik ook dank verschuldigd aan de onderzoeksschool NUTRIM, voor het raamwerk waarin ik mijn promotieonderzoek heb mogen vitvoeren.

DSM, in het bijzonder Maaike en Luppo, bedankt dat ik degene was die het AN-PEP project mocht uitvoeren. Het was een fijne samenwerking met mooie resultaten, we hebben zelfs de nationale en internationale pers gehaald!

Ik had mijn onderzoeksprojecten niet kunnen uitvoeren zonder hulp van alle MDL-artsen, MDL-assistenten, Ton Mestrom en het endoscopieteam. Hartelijk dank voor jullie hulp en geduld bij het afnemen van vele biopten in onvoorbereide darmen.

Dan niet te vergeten, Mietsie en Elly. Altijd gezellig om even bii jullie langs te lopen en daarbii direct een antwoord te krijgen op al mijn vragen. Tiny, dank voor al je hulp bij de lay-out van mijn proefschrift. Ik ben trots op ons eindresultaat.

Kira: bedankt voor het ontzettend gave design van dit boekie en de presentatie. Ik geloof in je!

Miin promotietiid was niet half zo leuk geweest zonder een fantastische groep onderzoekscollega's. Mariëlle, Eveline, Kirsten P., Kirsten v/d B., Elhaseen, Tim, Bas, Zsa Zsa, Lisa, Pauline, Marin, Martine, Montserrat, Daisy, Annemiek, Annick, Selwyn, Dirk en Bram, bedankt voor de gezellige lunches, borrels, congressen en de interesse in de voortgang van mijn projecten. Daniel, Samefko en Mark: bedankt voor het onvergetelijk maken van mijn eerste DDW in San Diego. Mark, ik wil jou in het bijzonder bedanken voor alle hulp tijdens de afgelopen jaren. Je leerde me duodenumsondes plaatsen, galblaasecho's maken en kwam menig keer opdraven als er weer een infuus geprikt moest worden. Zlatan, Givan, Marlou, Fedde, Maarten, Steven, Fabienne, Sander en Paul, met een grote griins denk ik terug aan Orlando 2013. De avondies in Senõr Frog's en de snake op onze hotelgang ziin mijn favorieten. Wanneer is de volgende reünie? Roel, roomie, zoals je weet waren Chantal en ik niet heel blii toen we hoorden dat iii op onze kamer erbii kwam. Maar gelukkig pakte het biizonder goed uit. Met jou erbii waren we altiij gegarandeerd van een luisterend (rood) oor en kritisch, doortastend mannencommentaar. Hao Ran, Harry, het laatste half jaar van mijn promotie vrolijkte iij onze werkkamer nog even op. Bedankt voor je grappige verhalen, de moestuinties en de verkeerd geadresseerde WhatsApp berichten. 
Ook wil ik mijn collega's bij BioActor bedanken voor alle hulp bij de verschillende onderzoeksprojecten. In het bijzonder Yala, bij bijna ieder project in dit boekje ben je wel betrokken geweest. Heel veel succes met jouw eigen promotietraject.

Mestreechter maedjes: Cor, lef, Jans, Maart en Ray. Een mengeling van meisjes, onafscheidelijk sinds jaar 1 van onze studie. Maandagavond, 'Greys Anatomy'-avond! Niks kwam hiertussen, zelfs geen man. Woensdagavond borrelen en op stap. Vanaf de coschappen werd deze doordeweekse stapavond gewoon verplaatst naar het weekend. We hebben samengewoond en zijn naar de meest prachtige oorden afgereisd. Bedankt voor deze vriendschap, voor het feit dat ik altiid alles met jullie kan delen, voor de goede etenties en gezellige borrels, de mooie feesties en fantastische vakanties. Ik mis onze maandagavonden nu al een tijdje, maar wees gerust, ik kom gewoon terug.

Lieve An, ook jou hebben we sinds kort weer terug in het Maastrichtse. Fijn dat je er weer bent en dat we elkaar weer wat vaker zien.

En dan niet te vergeten de oude garde van O.D.D. Missdaad: Cerès, Dree, Inge, Kim, Kira en Luus. Samen a-tijd gelopen en actief lid van O.D.D. Missdaad geweest. Zonder jullie had ik nooit zo'n fantastische studententiid gehad. Inmiddels een stuk rustiger, maar niet minder gezellig. Fijn dat we na al die jaren nog steeds zo close zijn. Ook al is het tegenwoordig niet meer zo vaak, ik geniet iedere keer weer van onze koffiedates, etenties en borrels. Lieve Bel, ook jou wil ik bedanken voor de fijne vriendschap. Ondanks dat we elkaar niet zo vaak zien weten we altiid wel van elkaar wat ons op dat moment bezighoudt. Laten we vooral niet ophouden met het plannen van leuke festivals.

Chantal en Ellen, allerliefste paranimfen, ik ben heel blij dat jullie naast mij staan deze belangriike dag, maar viteraard hebben jullie ook veel voor mij betekend in de afgelopen jaren.

Chantal, onze eerste avonturen samen beleefden we in Nepal. De vierdaagse trekking, de jungletocht en de achtervolging door een neushoorn. Erna volgden er gelukkig nog vele andere. We deden gezamenlijk onze WESP/GEZP bij de MDL, en begonnen tegelijk aan onze promotie. We werden naast kamergenoten en collega's ook goede vriendinnen, reisbuddy's, feestmaaties en heuse wijnkenners. Ik benijd je om jouw doorzettingsvermogen, gedrevenheid en doelgericht werken. Maar lieve Chantal, wees alsjeblieft niet altijd zo streng voor jezelf. Wat was ik trots dat ik zo'n 1,5 jaar geleden naast jou als paranimf mocht staan! Ik kijk ernaar uit dat we binnenkort weer collega's zijn.

Ellen, ik leerde je kennen als een enthousiaste, leergierige, kritische en assertieve control freak. Heerlijk dat dat mijn eerste stagiaire was. Ik was destijds nog niet zo goed in delegeren, maar al snel durfde ik veel onderzoekstaken aan jou over te laten. Sorry, ze waren niet altiid even leuk of dankbaar (denk aan de keren dat iij weer met een portie 
urine of ontlasting in je rugzak op pad ging), maar nooit hoorde ik je klagen en altiid deed je je taken met evenveel toewijding. Ik herinner me vooral dat we, ondanks het meestal vroege tijdstip, altijd veel lol hadden. Heel blij was ik dus ook dat je niet veel later m'n directe collega werd bij de MDL. We hebben elkaar nog veel geholpen in de afgelopen jaren met onze afzonderlijke studies. En naast collega werd je ook een goede vriendin, mijn motivatie om te gaan spinnen en bestudeerden we samen de kunde van de wijn.

Lieve Jorrit, mijn grote kleine broer. Vroeger waren we onafscheidelijk; speelde ik met de poppen, wilde iii ook; droeg ik nagellak, moest iii ook; lustte ik ineens paprika, vond ii het ook lekker. Toen we ouder werden, gingen we ieder onze eigen weg. Zie hier, ik ben (bijna) gepromoveerd en werk in het ziekenhuis; iij woont in Azië en runt je eigen internetbedrijf. Twee totaal verschillende werelden, maar toch voor altijd verbonden door onze lieve ouders en mooie jeugdherinneringen. Ik mis je soms wel, zou willen dat we weer meer herinneringen samen zouden maken. Maar het is goed zo. Ik ben trots op jou en op alles dat je hebt bereikt.

Papa en mama, lieve ouders, jullie zijn de oorsprong van dit alles. Ik zou natuurlijk nooit gepromoveerd zijn als jullie me niet op deze wereld gezet hadden, en het zou me ook nooit gelukt zijn zonder jullie onvoorwaardelijke liefde, opvoeding, steun, vertrouwen en interesse. Ook al is het soms lastig uitleggen wat promoveren nu exact inhoudt, altiij ziin jullie even geïnteresseerd, trots en enthousiast. Sorry mam, ik had je eigenlijk al eerder een samenvatting van m'n promotie beloofd, maar bii deze verwijs ik je graag naar het vorige hoofdstuk. Pap en mam, bedankt voor alles, ik ben er trots op dat jullie mijn ouders zijn en hoop nog lang van jullie te genieten.

Lieve Bas, lief vriendje.

Bedankt dat iij in mijn leven bent. Ik vind je prachtig. Ik bewonder je doorzettingsvermogen, daadkracht en eerlijkheid, je vermogen tot relativeren, het feit dat je niet bang bent om fouten te maken en niet doet aan twijfelen. Je geeft me de ruimte en het vertrouwen mezelf te ontwikkelen. En allerbelangriikst, je bent er voor me, iedere dag opnieuw. Ik hou van je. 
244 